SAIL 21

\title{
Linguistica Educativa e contesti migratori
}

Antonella Benucci, Giulia I. Grosso

e Viola Monaci

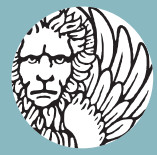

Edizioni

Ca'Foscari

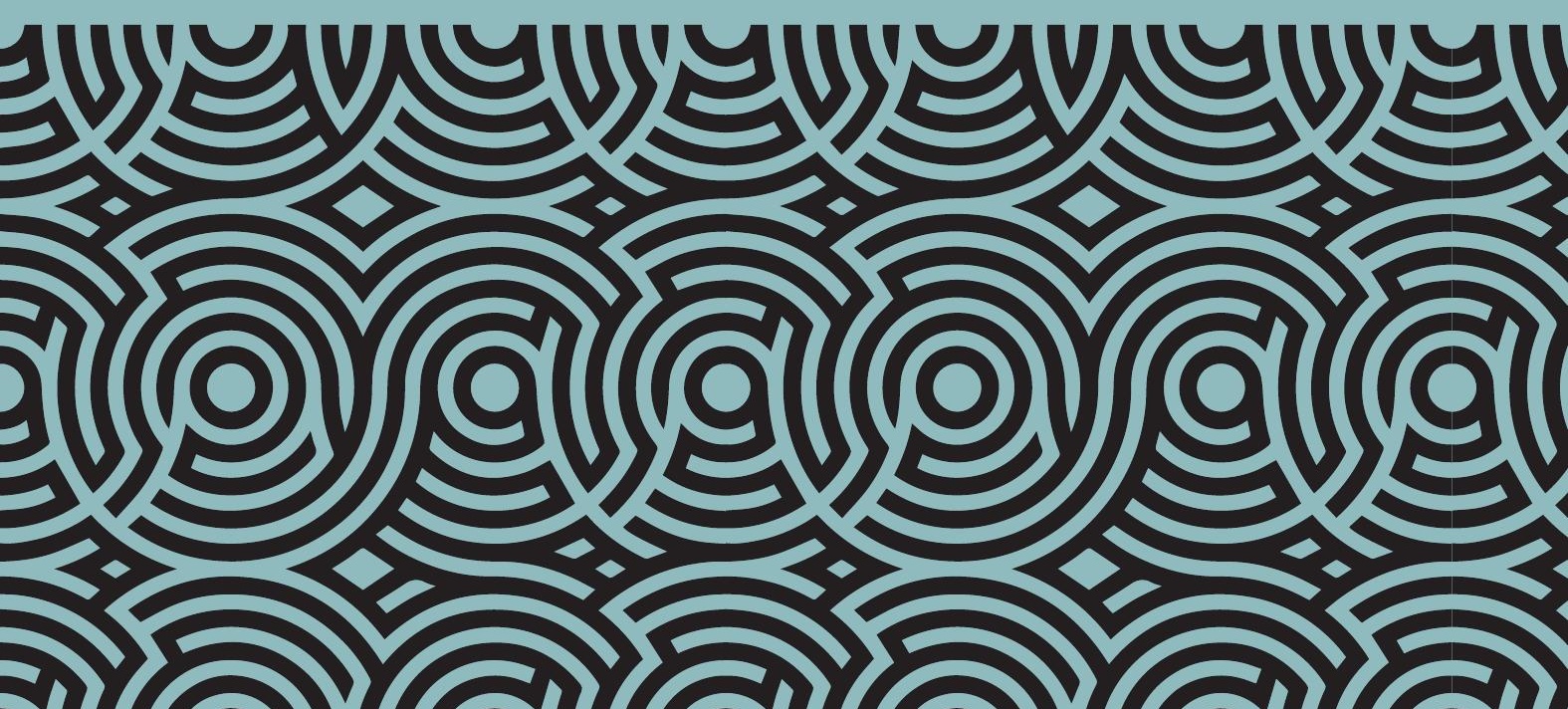


Linguistica Educativa e contesti migratori

\section{SAIL}

Studi sull'apprendimento e l'insegnamento linguistico

Serie diretta da $\mid$ A series edited by Paolo E. Balboni

21

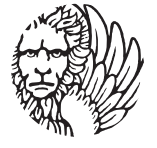

\section{Edizioni}

Ca'Foscari 


\section{SAIL Studi sull'apprendimento e l’insegnamento linguistico}

Comitato scientifico Simona Bartoli Kucher (UniversitätGraz, Österreich) Antonella Benucci (Università per Stranieri di Siena, Italia) Marilisa Birello (Universitat Autònoma de Barcelona, España) Fabio Caon (Università Ca' Foscari Venezia, Italia) Enrico Cecconi (University of Bath, UK) Carmel M. Coonan (Università Ca' Foscari Venezia, Italia) Diego Cortes (California State University, Long Beach, USA) Bruna Di Sabato (Università degli Studi Suor Orsola Benincasa, Napoli, Italia) Radica Nikodinovska (Methodius University, Skopje, Makedonija) Matteo Santipolo (Università dagli Studi di Padova, Italia) Graziano Serragiotto (Università Ca' Foscari Venezia, Italia) Maria Yashina (Moscow Business University, Russia) Nives Zudič (Univerza na Primorskem, Koper, Slovenija

Comitato di redazione Paolo E. Balboni (Università Ca’ Foscari Venezia, Italia) Carlos Alberto Melero Rodríguez (Università Ca'Foscari Venezia, Italia) Marco Mezzadri (Università di Parma, Italia) Anna Lia Proietto Basar (Yıldız Teknik Üniversitesi, İstanbul, Türkiye) Rita Scotti (Juraj Dobrila University of Pula, Croatia) Enrico Serena (Ruhr-Universität Bochum, Deutschland) Antonio Ventouris (Aristotle University of Thessaloniki, Greece)

Revisori DaliaAbdullah (Ain Shams University, Cairo, Egypt) Andrea Balbo (Università degli Studi di Torino, Italia) Elena Ballarin (Università Ca'Foscari Venezia, Italia) Mirela Boncea (West University of Timisoara, Romania) Elisabetta Bonvino (Università degli Studi Roma Tre, Italia) Giovanna Carloni (Università degli Studi di Urbino «Carlo Bo», Italia) Vanessa Castagna (Università Ca' FoscariVenezia, Italia) Veronique Castellotti (Université «François-Rabelais», Tours, France) Stefania Cavagnoli (Università degli Studi Roma «TorVergata», Italia) Paola Celentin (Università degli Studi di Verona, Italia) Cristiana Cervini (Alma Mater Studiorum Università di Bologna, Italia) Edith Cognigni (Università di Macerata, Italia) Daria Coppola (Università per Stranieri di Perugia, Italia) Elisa Corino (Università degli Studi di Torino, Italia) Michele Daloiso (Università degli Studi di Parma, Italia) Mariapia D’Angelo (Università «G. d'Annunzio» Chieti-Pescara, Italia) Maddalena de Carlo (Università di Cassino, Italia) Paolo Della Putta (Università del Piemonte Orientale, Italia) Anna de Marco (Università della Calabria, Italia) Paola Desideri (Università degli Studi «G. D’Annunzio» Chieti Pescara, Italia) Vesna Deželjin (University of Zagreb, Croatia) Pierangela Diadori (Università per Stranieri di Siena, Italia) Emilia Di Martino (Università degli Studi Suor Orsola Benincasa, Napoli, Italia) Giuliana Fiorentino (Università degli Studi del Molise, Italia) Francesca Gallina (Università di Pisa, Italia) Roberta Grassi (Università degli Studi di Bergamo, Italia) Giulia Grosso (Università per Stranieri di Siena, Italia) Amina Hachoulf (Badji Mokhtar University, Annaba, Algeria) Elisabetta Jafrancesco (Università degli Studi di Firenze, Italia) Marie Christine Jamet (Università Ca' Foscari Venezia, Italia) Zorana Kovacevic (University of Banja Luka, Bosnia and Herzegovina) Matteo La Grassa (Università per Stranieri di Siena, Italia) Terry Lamb (University of Westminster, London, UK) Liliana Landolfi (Università degli Studi di Napoli «L'Orientale», Italia) Maslina Ljubicic (University of Zagreb, Croatia) Ivan Lombardi (University of Fukui, Japan) Geraldine Ludbrook (Università Ca' Foscari Venezia, Italia) Maria Cecilia Luise (Università degli Studi di Udine, Italia) Sabrina Machetti (Università per Stranieri di Siena, Italia) Sandra Mardešić (University of Zagreb, Croatia) Carla Marello (Università degli Studi di Torino, Italia) Marcella Maria Mariotti (Università Ca' Foscari Venezia, Italia) Giuseppe Maugeri (Università Ca' Foscari Venezia, Italia) Patrizia Mazzotta (Università degli Studi di Bari «Aldo Moro», Italia) Marcella Menegale (Università Ca’ Foscari Venezia, Italia) Darja Mertelj (University of Ljubljana, Slovenia) Nikita Mihaljevic (University of Split, Croatia) Eliana Moscarda Mirković (Juraj Dobrila University of Pula, Croatia) Anthony Mollica (Brock University, St. Catharines, Ontario, Canada) Johanna Monti (Università degli Studi di Napoli «L'Orientale», Italia) David Newbold (Università Ca' Foscari Venezia, Italia) Paolo Nitti (Università degli Studi dell'Insubria, Italia) Alberta Novello (Università degli Studi di Padova, Italia) Cristina Onesti (Università degli Studi di Torino, Italia) Gabriele Pallotti (Università degli Studi di Modena e Reggio Emilia, Italia) Elisabetta Pavan (Università degli Studi di Padova, Italia) Ruggiero Pergola (Università degli Studi di Bari «Aldo Moro», Italia) Gianfranco Porcelli (Università Cattolica del Sacro Cuore, Milano, Italia) Rosa Pugliese (Alma Mater Studiorum Università di Bologna, Italia) Simonetta Puleio (UniversitätStuttgart, Deutschland) Federica Ricci Garotti (Università degli Studi di Trento, Italia) Fabio Ripamonti (University of South Bohemia in České Budějovice, Czech Republic) Flora Sisti (Università degli Studi di Urbino «Carlo Bo», Italia) Camilla Spaliviero (Università Ca' Foscari Venezia, Italia) Antonio Taglialatela (Università degli Studi della Tuscia, Viterbo, Italia) Giulia Tardi (Università degli Studi di Firenze, Italia) Simone Torsani (Università degli Studi di Genova, Italia) Victoriya Trubnikova (Università degli Studi di Padova, Italia) Ada Valentini (Università degli Studi di Bergamo, Italia) 


\section{Linguistica Educativa e contesti migratori}

Antonella Benucci, Giulia I. Grosso, Viola Monaci

Venezia

Edizioni Ca' Foscari - Digital Publishing 2021 
Linguistica Educativa e contesti migratori

Antonella Benucci, Giulia I. Grosso, Viola Monaci

(c) 2021 Antonella Benucci, Giulia I. Grosso, Viola Monaci per il testo

(C) 2021 Edizioni Ca' Foscari - Digital Publishing per la presente edizione

\section{(c) (i)}

Quest'opera è distribuita con Licenza Creative Commons Attribuzione 4.0 Internazionale This work is licensed under a Creative Commons Attribution 4.0 International License

OPEN

Qualunque parte di questa pubblicazione può essere riprodotta, memorizzata in un sistema di recupero dati o trasmessa in qualsiasi forma o con qualsiasi mezzo, elettronico o meccanico, senza autorizzazione, a condizione che se ne citi la fonte.

Any part of this publication may be reproduced, stored in a retrieval system, or transmitted in any form or by any means without permission provided that the source is fully credited.

Certificazione scientifica delle Opere pubblicate da Edizioni Ca' Foscari - Digital Publishing: il volume pubblicato ha ottenuto il parere favorevole da parte di un gruppo di valutatori esperti della materia selezionati dal Comitato editoriale.

Scientific certification of the works published by Edizioni Ca' Foscari - Digital Publishing: the volume has received a favourable opinion by a group of subject-matter experts selected by the Editorial Board.

Edizioni Ca' Foscari - Digital Publishing

Fondazione Università Ca' Foscari | Dorsoduro 3246 | 30123 Venezia

https://edizionicafoscari.unive.it/|ecf@unive.it

la edizione 2021

ISBN 978-88-6969-570-4 [ebook]

ISBN 978-88-6969-571-1 [print]

This volume is funded by the project COMMIT: Facilitating the integration of resettled refugees in Croatia, Italy, Portugal and Spain, funded by EAMIF 2017 Call for Proposals on Integration of Third-Country Nationals (AMIF-2017-AG-INTE) priority 3: Pre-departure and post-arrival support for the integration of persons in need of international protection who are being resettled from a third country including through volunteering activities, leading partner: OIM Italy.
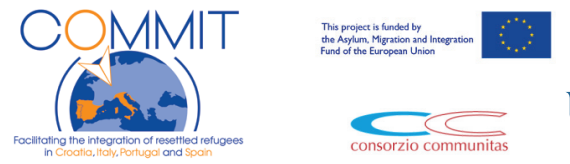

EONDAZIONEADECCO

PER LE PARI OPPORTUNITÁ

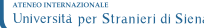

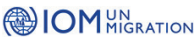

Linguistica Educativa e contesti migratori / Antonella Benucci, Giulia I. Grosso, Viola Monaci - 1. ed. - Venezia: Edizioni Ca' Foscari - Digital Publishing, 2021. - 228 pp.; $23 \mathrm{~cm}$. - (SAlL; 21). - ISBN 978-88-6969-571-1

URL https://edizionicafoscari.unive.it/it/edizioni/libri/978-88-6969-571-1/

DOI http://doi.org/10.30687/978-88-6969-570-4 


\section{Linguistica Educativa e contesti migratori}

Antonella Benucci, Giulia I. Grosso, Viola Monaci

\section{Abstract}

The volume, produced within the framework of the COMMIT project "Fostering the Integration of Resettled Refugees in Croatia, Italy, Portugal and Spain", concerns the current European situation, and in particular the teaching of $\mathrm{L} 2$ in its relations and interdisciplinary exchanges with other scientific fields dealing with migratory phenomena; therefore, starting from the COMMIT experience, it offers a wide perspective, going beyond the borders of the countries involved in the project and identifying good practices that can be replicated in different territorial and social contexts to ensure successful social inclusion of newly arrived citizens.

COMMIT is a project funded by the European Commission (DG HOME), co-financed by the Ministry of Interior and the Project Partners and managed by the Mediterranean Coordination Office of the International Organization for Migration (IOM), in Italy. The project was implemented in collaboration with the IOM Missions in Croatia, Portugal and Spain, together with the Communitas Consortium, the Adecco Foundation for Equal Opportunities and the University for Foreigners of Siena (UNISTRASI). The project activities were implemented from 1 January 2019 to 30 April 2021.

The project, based on the idea that successful integration of resettled refugees occurs both by putting in place certain structural conditions and by promoting mutual exchange between resettled refugees and their host communities, aimed to support their integration into their new communities, with a special focus on women and young refugees as particularly vulnerable groups. A secure humanitarian migration route to the European Union launched in 2013 is targeted at refugees who are beneficiaries of resettlement. Several Member States, including Croatia, Italy, Portugal and Spain, have therefore established or strengthened their national resettlement and humanitarian admission programmes for resettled refugees of Syrian, Eritrean, Ethiopian or Sudanese origin. In preparation for resettlement, beneficiaries participate in a series of pre-departure cultural orientation activities. Among them, training in L2 language and culture plays a crucial role.

The book hence tries to offer answers to the many challenges that characterise the field of language education in contexts marked by the presence of migrants from an interdisciplinary perspective.

It provides for effective solutions for an inclusive language education, attentive to 'vulnerable' subjects, paying attention to the interweaving of complex individual, social, cultural and economic contexts, such as school and university training courses and reception and resettlement programmes in host societies.

In particular, the current situation in Italy, regarding both teaching L2 in a school context and teaching modern languages to adult foreigners, is still lacking in interdisciplinary relations and exchanges between language teaching and other scientific fields dealing with migratory phenomena. However, in recent years a particular sensitivity and empathy towards linguistic and cultural contact have developed.

Keywords Vulnerable subjects. Language education. Interlanguage and interculture. Inclusivity. Plurilingualism. 



\section{Sommario}

\section{Premessa}

Antonella Benucci, Giulia I. Grosso, Viola Monaci

1 Principi di base dell'Educazione degli adulti immigrati

Antonella Benucci

2 Comunicazione efficace in contesti interlinguistici e interculturali

Antonella Benucci

3 Consapevolezza interculturale e interlinguistica: riflettere sulle lingue e culture dei beneficiari Giulia I. Grosso

4 Aspetti psicologici della migrazione e gestione dei conflitti

Viola Monaci

5 Problematiche e soluzioni per apprendenti analfabeti e per classi ad abilità differenziate Giulia I. Grosso

6 Tecniche e strumenti per la gestione della relazione mentor-rifugiato

Viola Monaci

\section{Bibliografia}





\section{Premessa}

Antonella Benucci

Università per Stranieri di Siena, Italia

Giulia I. Grosso

Università degli Studi di Cagliari, Italia

Viola Monaci

Università per Stranieri di Siena, Italia

Il presente volume nasce nell'ambito del progetto COMMIT: Facilitating the integration of resettled refugees in Croatia, Italy, Portugal and Spain.

COMMIT è un progetto finanziato dalla Commissione Europea (DG HOME), co-finanziato dal Ministero dell'Interno e dai partners di Progetto e gestito dall'Ufficio di Coordinamento per il Mediterraneo dell'Organizzazione Internazionale per le Migrazioni (OIM), ${ }^{1}$ in Italia. Il progetto è stato realizzato in collaborazione con le missioni OIM in Croazia, Portogallo e Spagna, insieme al Consorzio Communitas, Fondazione Adecco per le Pari Opportunità e all'Università per Stranieri di Siena (UNISTRASI). Le attività progettuali sono state realizzate dal $1^{\circ}$ gennaio 2019 al 30 aprile 2021.

Il progetto, basato sull'idea che l'integrazione dei rifugiati reinsediati avvenga con successo sia per la messa in atto di determinate condizioni strutturali, sia per la promozione dello scambio reciproco tra i rifugiati reinsediati e le comunità ospitanti, ha inteso sostenerne l'integrazione nelle loro nuove comunità, con particolare attenzione a donne e giovani rifugiati, quali categorie particolarmente

1 In questo volume si usa la sigla italiana OIM facendo presente che corrisponde all'inglese IOM (International Organization of Migration). 
vulnerabili. È destinato a rifugiati beneficiari di resettlement (reinsediamento) un percorso sicuro di migrazione su base umanitaria verso l'Unione Europea lanciato nel 2013. Numerosi Stati membri, tra i quali Croazia, Italia, Portogallo e Spagna, hanno dunque istituito o rafforzato i propri programmi nazionali di reinsediamento e di ammissione su base umanitaria di rifugiati reinsediati di origine siriana, eritrea, etiope o sudanese. In preparazione al reinsediamento, i beneficiari partecipano ad una serie di attività di orientamento culturale pre-partenza.

Le misure poste in essere dai Paesi europei per quel che concerne l'accoglienza e l'integrazione, sono diverse, tuttavia, alcuni elementi comuni possono essere individuati per far fronte all'accoglienza di migranti arrivati in maniera spontanea. I rifugiati reinsediati, pur avendo esperienze migratorie simili tra di loro, presentano bisogni, anche formativi, estremamente diversi a seconda della loro condizione individuale (ad esempio sesso, età, condizioni di disabilità e di salute, traumi subiti, livello di scolarizzazione/alfabetizzazione ecc.). È fondamentale, dunque, che tali bisogni individuali siano prontamente identificati, anche nella fase precedente alla partenza, per garantirne il seguito ed evitare l'aggravarsi di determinate situazioni di vulnerabilità. Oltre alla predisposizione di un sistema ben strutturato, che sappia far fronte in maniera adeguata ai bisogni dei beneficiari, l'integrazione dipende, inoltre, dalla narrazione su migranti/ rifugiati nel dibattito pubblico, nonché dalla percezione dei medesimi rispetto alla propria accettazione nella società di accoglienza. Il progetto COMMIT, dunque, intende rafforzare il raccordo tra la fase pre-partenza e quella post-arrivo del processo di reinsediamento, potenziando l'orientamento pre-partenza (PDO) e concentrandosi sulle vulnerabilità individuali, allo scopo di rafforzare la capacità di accoglienza delle comunità locali in Croazia, Italia, Portogallo e Spagna.

In questo contesto, il presente volume cerca quindi di offrire risposte alle molteplici sfide che caratterizzano tutti gli ambiti nei quali si realizza l'inclusione sociale dei migranti attraverso l'Educazione Linguistica, da una prospettiva interdisciplinare e ricercando soluzioni efficaci per soggetti 'svantaggiati', con una particolare attenzione all'intreccio di complesse dinamiche individuali, sociali, culturali ed economiche.

Il focus del volume riguarda la realtà europea attuale, e in particolare la didattica delle L2 nei suoi rapporti e scambi interdisciplinari con altri ambiti scientifici che si occupano di fenomeni migratori; partendo quindi dall'esperienza di COMMIT offre una prospettiva ampia, andando oltre i confini dei Paesi coinvolti nel progetto e identificando buone pratiche che possano essere replicate in contesti territoriali e sociali diversi per garantire un'inclusione sociale di successo dei cittadini neoarrivati. 


\section{Principi di base dell'Educazione degli adulti immigrati}

Antonella Benucci

Università per Stranieri di Siena, Italia

Sommario 1.1 Panoramica europea. - 1.2 Linguistica Educativa e Lifelong learning. 1.3 Didattica per soggetti svantaggiati.

\subsection{Panoramica europea}

In Europa il concetto di Educazione degli adulti si diversifica da Paese a Paese e si realizza in forme e con strategie che rispondono alle politiche nazionali e al concetto di educazione prevalente nelle diverse realtà, pur all'interno di comuni linee di azione come il Consiglio europeo di Lisbona (2000) o il Memorandum sull'Istruzione e l'educazione permanente (Commissione delle Comunità Europee 2000).

Il moderno concetto europeo di Educazione degli adulti riguarda sempre meno i percorsi di alfabetizzazione di persone che non hanno avuto l'opportunità di concludere la loro formazione in L1 o come attività di occupazione del tempo libero (per es. in Scozia) e si configura sempre più come azione di sostegno per immigrati, per il loro inserimento nella società che li ospita o per la (ri)qualificazione professionale. Nel tempo si è concretizzato in corsi di varia durata, ma accomunati da flessibilità, e organizzati sia in contesti formali che non formali di formazione, in presenza o a distanza (per es. in Francia l'insegnamento erogato dal CNED - Centre national d'enseignement à distance o in Spagna dal CIDEAD - Centro para la Innovación y Desarrollo de la Educación a Distancia), di lunga tradizione (come i 
CTP divenuti CPIA italiani o le Vlokshochschulen tedesche), per aree di specializzazione (Mechanics Institutes anglosassoni), per competenze in lettura o scrittura. ${ }^{1}$ Quanto alle caratteristiche dei destinatari di tali corsi nei Paesi dell'Europa meridionale (Francia, Spagna, Italia, Grecia, Portogallo, Malta), i residenti nati all'estero presentano livelli di istruzione e di competenza in L2 bassi (il livello più basso di competenza in lettura e scrittura si riscontra in Italia) e le differenze più rilevanti tra livelli di competenza linguistica tra nativi e non nativi sono invece in Svezia e in Finlandia. I pochi esempi riportati sono già sufficienti per confermare che esistono notevoli elementi di disparità tra i diversi Paesi europei.

Inoltre, non tutti gli Stati hanno interpretato alla stessa maniera la promozione del plurilinguismo e organizzato un'offerta linguistica che tenga conto dei diversi tipi di pubblico adulto. Malgrado le considerazioni di Calvet (2002) sul 'mercato delle lingue' risalgano a circa venti anni fa, lo studio di più lingue europee spesso si traduce in iniziative rivolte principalmente a corsi di potenziamento in inglese (seguiti da francese, spagnolo, russo e tedesco) portando di conseguenza ad un appiattimento delle variabili comunicative per loro natura fortemente influenzate da aspetti non linguistici, ma piuttosto di genere culturale, e che quindi non possono essere pienamente veicolate solo con la traduzione di nomenclature. Questo orientamento generalizzato si riflette anche nelle scelte linguistiche di mediazione che spesso utilizzano l'inglese come lingua franca tra mediatore e mediato, dando priorità all'esperienza di migrazione del mediatore piuttosto che agli aspetti linguistico-culturali, con i conseguenti prevedibili rischi connessi. D'altronde, in alcuni ambiti di mediazione (come quello sanitario) ricorrere a persone della stessa comunità può essere rischioso e non rispettoso della privacy ma ancor più problematico è che il mediatore condivida con il mediato unicamente il codice linguistico ma possieda una cultura e ancor peggio un credo e convinzioni sociali differenti.

Il primo aspetto da esaminare è il fallimento della Strategia Europa 2020 (Comunicazione della Commissione Europa 2020), già annunciato nel 2004 dal Rapporto KOK (2004) che aveva denunciato il divario tra Europa e Stati Uniti in tema di produttività, soprattutto per le differenze legate alla disponibilità e all'impiego di infrastrutture e servizi ICT (Information and Communications Technology; in italiano, TIC o tecnologie dell'informazione e della comunicazione). Una strategia quale quella europea, che si pone l'obiettivo di favorire uno sviluppo basato sulla conoscenza diffusa e innovativa, sulla sostenibilità delle risorse, sull'inclusione e l'uguaglianza sociale, costituisce un importante investimento per il futuro da un punto di

1 Per ulteriori informazioni si veda Eurydice 2005; Commissione Europea/EACEA/ Eurydice 2015. 
vista teorico, ma sul piano pratico non è semplice da realizzare. Anche l'obiettivo di promuovere il riconoscimento dell'apprendimento non formale e informale sembra essere fallito.

L'antecedente della Strategia è il Libro Bianco sul dialogo interculturale (White Paper 2008) che aveva lo scopo di fornire un quadro concettuale e una guida di indirizzo per istituzioni, comunità locali, società civile, comunità religiose e immigrati per il confronto con la governance democratica della diversità culturale partendo dal presupposto che il dialogo interculturale non può realizzarsi ove non si condividano valori fondamentali quali i diritti umani, la democrazia e il primato del diritto.

Si elencano sinteticamente alcuni aspetti e termini chiave affermati nel documento europeo, fondamentali anche per gli scopi di questa riflessione:

- il dialogo interculturale deve essere fondato sulla reciproca comprensione fra individui e gruppi che hanno origini e patrimonio etnico, culturale, religioso e linguistico differenti;

- multiculturalità e diversità culturale attengono all'esistenza di diverse culture e alla loro capacità di interagire in uno spazio specifico e in una determinata organizzazione sociale;

- la coesione sociale si riferisce alla capacità di una società di garantire il benessere di tutti i suoi membri, riducendo le disparità al minimo ed evitando le polarizzazioni;

- sono chiamati in causa i responsabili del potere pubblico e dei governi, le collettività locali e regionali, le organizzazioni della società civile, le comunità di migranti e quelle religiose, le organizzazioni culturali e dell'informazione, i giornalisti e i partner sociali;

- l'integrazione e l'inclusione formano un processo a doppio senso che implica l'attitudine delle persone a vivere insieme, nel pieno rispetto della dignità individuale, del bene comune, del pluralismo e della diversità, della non violenza e della solidarietà, nonché la loro capacità di partecipare alla vita sociale, culturale, economica e politica - tutelando i deboli e garantendo il diritto di essere diversi;

- gli immigrati possono partecipare pienamente alla vita del Paese di accoglienza ma devono conformarsi alle leggi e rispettare i valori fondamentali delle società.

Insistendo sulla indispensabilità del dialogo culturale il White Paper $(2008,18)$ affronta implicitamente anche il tema della comunicazione quando afferma che

Intercultural dialogue is therefore important in managing multiple cultural affiliations in a multicultural environment. It is a mechanism to constantly achieve a new identity balance, responding to new openings and experiences and adding new layers to identity 
without relinquishing one's roots. Intercultural dialogue helps us to avoid the pitfalls of identity policies and to remain open to the challenges of modern societies.

La comunicazione è in grado di produrre azioni per eliminare le barriere se si comprende l'altro, se si è in grado di dialogare con esso, ed è linguistica in primis servendo a veicolare contenuti religiosi, di genere, di riflessione critica, della libertà di pensiero, di pari opportunità. Un altro importante passo del documento mette in guardia contro il rischio del fallimento delle conversazioni interculturali dovuto a non condivisione o non conoscenza della lingua e afferma il diritto al plurilinguismo comprendente le lingue minoritarie immigrate:

Language is often a barrier to conducting intercultural conversations. The interculturalist approach recognises the value of the languages used by members of minority communities, but sees it as essential that minority members acquire the language which predominates in the state, so that they can act as full citizens. This chimes with the European Charter for Regional or Minority Languages, which argues that lesser-spoken languages should be protected from eventual extinction as they contribute to the cultural wealth of Europe, and that use of such languages is an inalienable right. At the same time, it stresses the value of multilingualism and insists that the protection of languages which enjoy minority usage in a particular state should not be to the detriment of official languages and the need to learn them. Language learning helps learners to avoid stereotyping individuals, to develop curiosity and openness to otherness and to discover other cultures. Language learning helps them to see that interaction with individuals with different social identities and cultures is an enriching experience. (White Paper 2008, 29)

Le migrazioni accompagnano lo sviluppo della storia degli esseri umani fin dalle epoche antiche. Spostarsi sui territori è una prerogativa sia dei singoli che dei gruppi, costituisce parte integrante della volontà di soddisfare i propri bisogni, di migliorare le proprie condizioni di vita. È dunque una qualità connaturata alla condizione umana, che ha permesso non solo la sopravvivenza, ma anche l'integrazione del mondo e il suo sviluppo. Le forme di questi fenomeni ricorrenti nella storia dell'umanità sono oggi attualizzate e amplificate dalla crescita demografica globale, dallo sviluppo delle tecnologie, dal rafforzamento della capacità di spostarsi grazie al più facile accesso ai mezzi di trasporto. Si tratta di condizioni nuove rispetto alle epoche passate che, insieme ai fattori concomitanti di ordine economico, culturale e sociale, ma anche climatico e ambientale, hanno impresso una forte accelerazione alla mobilità migratoria. 
Molti studi (ad es. Castles et al. 2020; Ambrosini 2011; O’Reilly 2012; Wihtol de Wenden 2017) che si occupano di indagare più a fondo la portata e le motivazioni di tali movimenti nell'epoca moderna considerano le migrazioni un fenomeno inevitabile (e una risposta strategica) in un mondo attraversato da crisi politiche ed economiche, segnato dalla diseguale distribuzione della ricchezza. Tenendo conto degli elementi annoverati tra i fattori migratori, a cui si aggiungono i fattori legati all'intensificazione e all'estensione delle zone interessate da guerre e da conflitti, è inevitabile ritenere che le migrazioni siano destinate ad aumentare nei prossimi anni, ipotesi avvalorata anche dalla stima dell'Organizzazione Internazionale per le Migrazioni (IOM 2020a; 2020b) secondo la quale, alle soglie del 2020, su oltre sette miliardi di abitanti del pianeta, oltre un miliardo di persone è in movimento, di cui circa il 3\% è costituito da migranti internazionali mentre 760 milioni sono migranti interni che si spostano entro i confini dello stesso Paese.

Tuttavia, se si guarda complessivamente il fenomeno in prospettiva planetaria, la migrazione riguarda una quota relativamente ridotta dell'umanità e l'emergere di reazioni di smarrimento e di minaccia nelle popolazioni dei Paesi di destinazione sembra piuttosto determinata da aspetti come la concentrazione in determinate aree di destinazione, la rapidità della formazione di nuovi flussi, le modalità drammatiche di una parte degli arrivi, le notizie diffuse da certi media.

Limitando l'analisi della situazione alla sola Europa si nota che le popolazioni con cittadinanza straniera più numerose sono maggiormente presenti in alcuni Paesi - Germania, Regno Unito, Italia, Spagna, Francia -, causa ne sono le posizioni geografiche, i livelli economico-produttivi o le politiche di accoglienza.

I flussi migratori attuali stanno ponendo dunque una serie di sfide agli Stati moderni, sia di origine, sia di partenza che di accoglienza: questa accelerata mobilità migratoria implica infatti una velocità di cambiamento sociale e culturale che, per quanto riguarda i Paesi sviluppati di destinazione, coglie spesso impreparati i vari attori sociali, i governi e le stesse popolazioni, generando paure, preoccupazioni e pregiudizi che rendono complessa e faticosa la convivenza tra diverse nazionalità ed etnie. Si tratta di sentimenti che devono essere compresi e analizzati, considerando che l'arrivo e l'insediamento di persone 'straniere' nel territorio di una nazione ha un forte impatto sulle modalità di instaurare e mantenere i rapporti sociali tra individui, nativi ${ }^{2}$ e immigrati.

I vari attori della comunicazione, in primis i migranti e i cittadini delle comunità dei Paesi d'arrivo, e contestualmente il mondo dell'in-

2 Si usa 'nativi' per brevità, alludendo a chi è originario del Paese in cui arriva l'immigrato; tuttavia il termine appare oggi inadeguato nel definire il concetto dato che sono 'nativi' anche i figli di immigrati. 
formazione, della sfera politica e istituzionale, del settore educativo e dell'istruzione, il mondo economico e dell'impresa, della società civile, giocano un ruolo fondamentale sia nella concretizzazione delle situazioni di marginalità sia nella formazione di narrazioni che si sono originate nei Paesi europei in seguito alle modifiche delle politiche migratorie dei governi, che con il tempo si sono orientate sempre più a considerare come prioritario il contenimento dei flussi dei migranti, potenziando gli apparati di sicurezza e le politiche di controllo, a scapito di politiche attive di integrazione nei territori. Non molti anni fa Vedovelli (2012) affermava che gli immigrati si muovono in realtà complesse, in cui il repertorio linguistico di origine viene sollecitato in tutte le sue componenti, e invitava ad una loro attenta analisi, mentre chi scrive metteva in guardia sulle complicazioni concomitanti degli aspetti culturali invocando una attenta considerazione nelle azioni educative alle coordinate culturali di tempo e spazio e ad altri aspetti come gli stereotipi, i pregiudizi, gli impliciti (Benucci 2008; 2015; Benucci, Biotti 2007). Purtroppo troppo spesso questo genere di riflessioni rimane circoscritto all'ambito accademico e non ha ricadute pratico-applicative.

Per governare le migrazioni non è sufficiente gestirne i flussi. Bisogna anche fare in modo che il migrante, soprattutto se vive condizioni di irregolarità, non finisca nel circolo vizioso di marginalità ed esclusione, che conduce facilmente sulla strada della devianza. Rischio, quest'ultimo, che può essere ridotto grazie ad adeguate politiche educative o rieducative (Benucci, Grosso 2017; Benucci 2014)

Uno dei fenomeni più inquietanti è il disagio che si origina frequentemente nelle dinamiche dell'incontro e della relazione tra nativi e immigrati, dando voce alle narrazioni delle comunità locali e dei migranti sia nei Paesi di origine, che nei Paesi di transito e di arrivo. Infatti il tema delle migrazioni è una questione 'socialmente viva' e modella sia la quotidianità dei discorsi privati sia quella dei discorsi pubblici, delle rappresentazioni nei media e nelle reti sociali, delle azioni e dei provvedimenti degli attori politici e dei responsabili istituzionali. Nei Paesi europei che sono, come già accennato, area di destinazione di una forte componente di flussi migratori si formano idee e convinzioni anche in base a come vengono 'raccontate' le migrazioni e presentati i migranti che sostengono atteggiamenti e comportamenti consequenziali. Spesso queste narrazioni semplificano e banalizzano - quando non distorcono - la complessità con cui si presenta il fenomeno migratorio che comprende una molteplicità di fattori di origine e una grande varietà di profili di persone interessate, e hanno effetti negativi sulle opinioni pubbliche, fomentando o amplificando paure, stereotipi, pregiudizi e contribuendo a rafforzare un sentimento ostile nei confronti dei migranti e di diffidenza da parte dei migranti nei confronti delle comunità di arrivo. Lo stereotipo è un'opinione precostituita, non acquisita sulla base di un'esperienza 
diretta, e scarsamente suscettibile di modifica, particolarmente difficile da sradicare quando riguarda gli immigrati. Più grande è la distanza culturale più difficoltoso è il reperimento di 'segnali culturali' dal contesto e dunque è più facile che si formino giudizi di valore che possono portare a visioni errate dell' ‘altro' fino al rifiuto totale della sua persona e della sua cultura. Gli stereotipi possono formarsi anche attraverso l'esposizione ai messaggi veicolati dai mass media o condivisi da una parte della collettività (di appartenenza o ospitante) ma il filtro e l'interferenza della cultura materna sono maggiori con l'aumentare della distanza culturale, pur essendosi oggi notevolmente ridotte le distanze culturali rispetto ai decenni passati.

Molti studi hanno affrontato il ruolo della narrativa in contesto migratorio (tra gli altri Boswell, Geddes, Scholten 2011; De Fina, Georgakopoulou 2021; Blinder 2015; De Fina, Baynham 2006): se i miti dell'antichità hanno strutturato le grandi narrazioni della civiltà, oggi i maggiori produttori di narrazioni sono i (social) media, parzialmente responsabili di come nelle società contemporanee si organizza il mondo e si generano immagini della realtà. Le esperienze del mondo sono necessariamente processate attraverso le narrazioni (cf. Bruner 1991) al cui interno quelle mediatiche, in particolare, producono e riproducono credenze sociali, suggeriscono standard di condotta, diffondono stereotipi e immagini degli altri. Troppo poco si sa della complessa macchina di accoglienza, dell'operato dei mediatori, di come si svolgono le pratiche burocratiche all'arrivo negli hotspot; le immagini che vengono offerte si fermano ai barconi, alle file per i controlli ma poco raccontano di cosa accade dopo e soprattutto delle persone, del loro aspetto umano, salvo dei casi di disturbo alla società di accoglienza.

Negli ultimi venti anni il discorso riguardo al fenomeno migratorio ha assunto dimensioni rilevanti e molteplici sfaccettature, diventando un argomento saliente per l'opinione pubblica, presente a più livelli nei diversi canali della comunicazione. Recenti studi sui sondaggi condotti nello stesso periodo si sono occupati della percezione e degli atteggiamenti delle persone riguardo alla migrazione e alle figure coinvolte nei fenomeni correlati ad essa. Tali studi hanno dimostrato una frequente distorsione della percezione della presenza degli immigrati riconducibile alla preoccupazione delle società di accoglienza relativamente ad aree ben specifiche della vita civile come, ad esempio, l'accesso ai servizi di pubblica utilità, l'aumento della disoccupazione e l'incidenza della criminalità. Tale distorsione è frutto principalmente della mancanza di informazione sull'argomento e sui suoi protagonisti, e può essere alimentata talvolta con l'intento di strumentalizzare l'opinione pubblica al fine di suffragare determinate ideologie politiche. Infatti non di rado la percezione dei migranti viene associata a quella dei criminali, tema tra i più spinosi tra quelli che riguardano la questione migratoria: già a partire dalla seconda 
metà degli anni Novanta del secolo scorso, con il notevole aumento dei flussi migratori nel continente europeo, si è infatti andata sviluppando nell'opinione pubblica la tendenza a considerare l'immigrazione come uno dei maggiori problemi di ordine pubblico, rafforzando l'idea di un legame migranti-criminalità e offrendo la giustificazione più 'convincente' all'atteggiamento negativo nei loro confronti. ${ }^{3}$

Nell'affrontare le narrazioni sul fenomeno migratorio il fatto di esplicitare anche il punto di vista di chi emigra impedisce di privilegiare il solo punto di vista della società d'approdo, che ha per lo più un'immagine dell'immigrazione come 'problema' ed 'emergenza', e consente anche di superare il rischio generale che deriva da un certo 'etnocentrismo sociologico' e proponente modelli di integrazione che non tengono conto nell'elaborazione delle narrative su quella che è realmente la vita dei soggetti migranti precedente (e conseguente) all'arrivo, perdendo di vista la loro soggettività e il bagaglio esperienziale e culturale con il quale essi tentano di inserirsi nel Paese d'approdo del loro progetto migratorio.

Uno studio del 2016 condotto da Open University (Gillespie et al. 2016) ha evidenziato l'importanza della tecnologia e dell'uso dei social nei viaggi dei migranti, sottolineando che le moderne risorse tecnologiche permetterebbero di sopperire alla mancanza di informazioni che spinge ad affidarsi a soluzioni illegali e pericolose, mentre il loro uso consapevole migliora le tappe dell'inclusione. Da tale studio emerge anche che la non condivisione di un codice comune con le società di arrivo è causa di malintesi, genera ulteriori stereotipi, ulteriore disagio e maggior isolamento. Nonostante vi sia un numero crescente di risorse digitali messe a disposizione dei rifugiati, molte di queste non danno voce ai diretti interessati e diffondono disinformazione, il grande pubblico sa poco di chi cerca di aiutarli con la sua opera di mediazione a cui generalmente non si dà voce. Le esperienze dei profughi, le politiche in materia e la loro implementazione stanno cambiando velocemente ed è necessario valutare come creare buone

3 La reclusione è chiusa al controllo pubblico e dei media: le notizie dal e sul carcere e sui detenuti sono sovente fornite - come mostrano alcuni studi sociologici (tra gli altri Ericson, Baranek, Chan 1987; Ericson 1995; Chermak 1995) - in risposta a preoccupazioni del grande pubblico e tendono a escludere dallo spazio pubblico la persona condannata pensando che il trattamento rieducativo di un imputato non sia interessante per il pubblico ma soprattutto dando un'immagine di ordine ripristinato in cui le persone 'libere' possano sentirsi al sicuro. D'altra parte, i dati dell'ultimo rapporto SPACE sulle Statistiche Penitenziarie Europee del Consiglio d'Europa del 2018 (Aebi, Hashimoto 2018), che fotografa la situazione del sistema penitenziario negli Stati membri europei, di World Prison Brief per alcuni altri Paesi non membri, così come quelli forniti da Eurostat, dimostrano che negli ultimi dieci anni la popolazione detenuta in Europa è diminuita (con alcune eccezioni), ma è aumentata la percentuale di stranieri tra i detenuti. La maggior parte di queste persone è detenuta per i reati di furto e spaccio di sostanze stupefacenti, reati che non comportano pene molto elevate, ma che hanno grande visibilità e risonanza presso l'opinione pubblica. 
pratiche per la gestione delle narrative delle e sulle migrazioni. Sono noti ormai la penetrazione di Internet, il numero di possessori di uno smartphone, l'uso sempre più massiccio delle app di messaggistica istantanea. Imparare, attraverso l'osservazione e la pratica, a esprimersi correttamente in ambienti diversi significa avere la possibilità di ottenere informazioni, di influire sulle scelte importanti, come dimostrano le consultazioni online promosse in vari ambiti della vita pubblica. In sintesi, significa imparare a esercitare con efficacia e consapevolezza la cittadinanza digitale e non.

Conseguenza di questo stato di cose è che le competenze (inter)linguistiche e interculturali sono indispensabili per una migliore convivenza tra società ospitanti e migranti ma devono essere acquisite con l'ausilio di figure professionali come quelle del settore educativo ancora troppo poco chiamate in causa dai governi e soprattutto con quelle dei mediatori.

Risulta inoltre chiaro come l'istruzione e la formazione abbiano un ruolo cruciale per il raggiungimento degli obiettivi di molte strategie e raccomandazioni europee, non soltanto la strategia di Lisbona, anche se sono incentrati su aspetti non immediatamente riconducibili ad esse come quelli relativi alla crescita economica, all'inclusione sociale e alla competitività. Oggi più che mai il ruolo dell'Educazione degli adulti diviene indispensabile per i programmi nazionali degli Stati europei: oltre che per permettere lo sviluppo di competenze necessarie per la realizzazione personale, anche per far sì che i processi migratori si attuino con successo e soddisfazione sia per coloro che migrano sia per chi riceve e accoglie queste persone nel proprio Paese permettendo un accesso al mondo del lavoro non clandestino e in condizioni dignitose e rispettose dei diritti umani. È indubbio che l'immigrazione possa controbilanciare almeno parzialmente l'invecchiamento della popolazione europea e apportare in determinati settori nuove competenze, oltre che manodopera. In particolare nel mondo del lavoro, come si è già avuto modo di segnalare (cf. Benucci 2014), la formazione linguistica degli immigrati non può essere intesa unicamente come risposta alle situazioni di emergenza legate ai bisogni di sopravvivenza comunicativa e neppure come necessità per soddisfare le misure normative in merito all'ottenimento del permesso di soggiorno. L'evitamento del fallimento del progetto migratorio è subordinato a vari fattori tra i quali non ultimo per importanza l'inserimento nel mondo del lavoro. L'immigrato lavoratore deve poter decodificare istruzioni spesso di vitale entità non soltanto per il corretto svolgimento dell'attività professionale ma anche per la sicurezza sul luogo di lavoro, o per comprendere la lingua della burocrazia. Fattori di natura pragmatica, sociolinguistica e culturale possono compromettere la riuscita degli scambi comunicativi in tale ambito: la competenza comunicativa in L2, così come di specifici aspetti culturali, risulta fondamentale non soltanto per trovare 
un impiego ma perché sia possibile conservarlo, migliorare le proprie condizioni sociali e interagire con i colleghi. Ai corsi di alfabetizzazione è dunque necessario affiancare interventi di formazione linguistica e culturale da attuare in specifici ambiti e contesti professionali. La progettazione di strumenti educativi, in primis linguistici (sillabi, curricoli, materiali didattici) ma anche teorico pratici (corsi di formazione specifici per insegnanti e tutor di azienda ecc.), ha il significato di investire negli elementi di sviluppo sociale e lavorativo offrendo agli stranieri percorsi di formazione linguistico-culturale spendibili per le esigenze lavorative. Wadsworth (2010) suppone che i motivi di bassa qualificazione dei lavoratori mobili UE si trovino nelle difficoltà di riconoscimento e di verifica formale delle qualifiche e delle competenze acquisite, nelle difficoltà linguistiche, oltre che nel fatto che i lavori cui si rivolgono i cittadini in mobilità dell'Unione Europea offrono salari più alti rispetto a quelli del loro Paese di origine, anche se in posti di lavoro meno qualificati di quelli che ricoprirebbero in patria.

Infatti, se la maggior parte degli immigrati adulti presenta lacune che possono impedire una integrazione in termini di competenze linguistiche e culturali, ove queste siano possedute non sono adeguatamente riconosciute ai fini delle attività professionali svolte di modo che nel mercato del lavoro le loro situazioni, in particolar modo quelle delle donne, non appaiono qualificate e sono inferiori a quelle dei cittadini dell'Unione Europea di pari livello.

Richiamando quanto affermato all'inizio di questo capitolo, si ribadisce che la definizione di Educazione degli adulti migranti varia da Paese a Paese anche in conseguenza delle politiche di accoglienza e delle tradizioni scolastiche e di formazione degli adulti, ambito in cui operano una moltitudine di organizzazioni governative e non, e su cui insistono normative diversificate - animation socio-culturelle in Francia, Folkeoplysning in Danimarca, continuous vocational training in Inghilterra, educazione popolare e Università popolare in Italia - tanto che l'OCSE ha riscontrato una serie di difficoltà nel determinare il concetto stesso di Educazione degli adulti.

Le caratteristiche degli immigrati in UE sono peggiori se si tratta di donne e possono essere riassunte come segue:

- la maggior parte delle donne provenienti da Paesi terzi è esiguamente attiva nel mercato del lavoro;

- una quota non indifferente delle donne provenienti da Paesi terzi è giunta in Europa per un ricongiungimento familiare;

- essendo cittadine di Paesi terzi non hanno gli stessi diritti dei migranti che provengono da altri Paesi UE (con particolare riferimento al permesso di lavoro e di soggiorno);

- la maggioranza della popolazione immigrata con necessità impellenti di istruzione è di genere femminile. 
Gli indicatori di Saragozza sul livello di integrazione degli immigrati elencano quattro aree di attenzione: inclusione sociale, cittadinanza attiva, occupazione e istruzione. Nei Paesi dell'Unione Europea che sono stati analizzati nell'indagine che ha prodotto lo sviluppo di tali indicatori (OECD; European Union 2018, Principali indicatori sull'integrazione degli immigrati) il numero di donne immigrate che ha un'occupazione è molto più basso di quello delle donne non migranti, autoctone, soprattutto in Germania, Svezia, Paesi Bassi e Belgio mentre in altri Paesi europei come Lussemburgo, Ungheria e Italia il divario tra autoctone e immigrata si attenua. Comunque il numero di donne immigrate con una occupazione resta ovunque al di sotto, seppur di non molto, di quello dei loro connazionali uomini.

La Commissione delle Comunità Europee nella Comunicazione Educazione degli adulti: non è mai troppo tardi per apprendere sposta l'attenzione verso l'Educazione degli adulti migranti, sfida prioritaria di una società europea della conoscenza:

L'educazione degli adulti può contribuire a far sì che l'immigrazione possa compiersi in modo da risultare vantaggiosa sia per i lavoratori migranti che per il paese di accoglienza. La maggior parte dei nuovi immigrati, anche coloro con un elevato livello di competenze, ha infatti notevoli esigenze in termini di conoscenze linguistiche e integrazione culturale. (Commissione delle Comunità Europee 2006, 4)

Ne sono conseguiti molte azioni e sforzi per l'ambito dell'alfabetizzazione in L2, della certificazione, della formazione all'interculturalità, svariati programmi e una mole di finanziamenti di progetti che tuttavia presentano ancora caratteri di episodicità, non omogeneità e inadeguatezza alle reali esigenze delle società europee, e soprattutto degli immigrati.

Soltanto con l'Agenda Europea rinnovata per l'apprendimento degli adulti (2011) si giunge al pieno riconoscimento che la coesione sociale non è possibile senza la creazione di adeguate condizioni di accesso all'apprendimento da parte di migranti, rifugiati e richiedenti asilo.

L'UE si è dotata negli ultimi venti anni circa di regolamenti, convenzioni, dichiarazioni e altri documenti finalizzati a garantire pari diritti e opportunità e occupabilità indipendentemente dalla razza, dal genere e dall'età, come le Direttive 2000/78/CE e 2000/43/CE (2000), il Trattato sull'Unione Europea (2012), la Direttiva sul principio della parità di trattamento fra le persone indipendentemente da razza e origine etnica (cf. Relazione 2021), che purtroppo nella maggior parte dei casi restano mere dichiarazioni di intenti e reiterano nel tempo, sotto forme e parole diverse, gli stessi obiettivi che si ritrovano anche nel Piano d'azione per l'integrazione e l'inclusione 20212027 (Commissione Europea 2020) accentuando l'attenzione verso le 
diverse forme dell'immigrazione di cui non vengono risolte in concreto le esigenze formative e di vita.

Il Fondo Asilo Migrazione e Integrazione (FAMI) ha finanziato nel tempo molti progetti ambiziosi che hanno raggiunto singoli ottimi risultati ma non hanno promosso l'effettiva Educazione degli adulti migranti perché, ancora una volta, soffrono del carattere episodico e individualistico che purtroppo caratterizza le azioni europee in questo ambito.

Come emerge da alcuni sondaggi, ancora una volta l'Europa si dota di proclami unitari di alto valore ma gli atteggiamenti verso la migrazione sono diversi da Stato a Stato e da zona a zona pur presentando alcune caratteristiche comuni come quella del riconoscimento dell'importanza che gli immigrati parlino la lingua dei Paesi ospitanti, possiedano titoli di studio elevati e abilità lavorative, ma soprattutto che si impegnino ad assumere lo stile di vita nazionale o si adeguino alle leggi.

Gli atteggiamenti verso l'immigrazione possono essere di diverso tipo ed essere correlati a specifici tipi di politiche di immigrazione, rivolti a discapito di determinati gruppi di immigrati, in dipendenza di atteggiamenti sedimentati nella storia di una nazione ecc. La letteratura teorica sul tema presenta punti di debolezza, è centrata sugli Stati Uniti, ed è permeata da endogeneità e da questioni metodologiche - soprattutto riguardo alla teoria del contatto che prevede che alcuni individui abbiano delle concezioni distorte sugli immigrati e che il contatto allenti tali distorsioni e pertanto renda gli individui maggiormente a favore dell'immigrazione: attività di mediazione tra nativi e non nativi possono produrre una conoscenza migliorata, maggiore empatia e un allentamento dell'ansia. Il contatto personale porta ad atteggiamenti a favore dell'immigrazione mentre il contatto non personale porta ad atteggiamenti contro l'immigrazione. Aspetti, tutti questi, da non tralasciare nell'implementazione di pratiche di educazione unitamente all'analisi dei differenti tipi di opinioni e atteggiamenti che si riscontrano in Europa: la Svezia è la nazione con il più alto grado di accettazione mentre l’Ungheria è quella con il grado più basso; l'Italia è al penultimo posto (segue l’Ungheria) per quanto riguarda l'accettazione gli immigrati dello stesso gruppo etnico.

Nella misurazione degli atteggiamenti verso gli effetti dell'immigrazione in aree differenti - economia nazionale, occupazione, welfare, crimine, cultura -, la Svezia è l'unico Paese in cui le persone non vedono l'immigrazione principalmente come una minaccia mentre ancora una volta l'Italia è il primo Paese in cui le persone temono gli effetti dell'immigrazione principalmente, come già accennato, nell'area del crimine. Inoltre, gli atteggiamenti verso l'immigrazione risultano più positivi tra i giovani e le persone maggiormente istruite, le donne e coloro che risiedono in area urbane; la fiducia nelle 
istituzioni, quella interpersonale, la soddisfazione economia e la sensazione di sicurezza influiscono altresì positivamente sugli atteggiamenti nei confronti dell'immigrazione. Inoltre, livelli più alti nell'uso di Internet portano ad atteggiamenti significativamente più positivi verso l'immigrazione.

Avere un titolo universitario, o comunque un alto livello di istruzione, riduce il timore dell'immigrazione e contribuisce significativamente ad atteggiamenti positivi verso l'immigrazione in generale, con l'eccezione dell'Italia e della Grecia.

La fiducia nelle istituzioni a livello nazionale contribuisce significativamente ad atteggiamenti maggiormente a favore degli immigrati: nei Paesi con un grado di tolleranza maggiore rispetto alla corruzione e in Paesi dove le persone percepiscono una maggiore diffusione della corruzione la paura degli immigrati è più alta, così come i Paesi con livelli più alti di orgoglio nazionale sono contro l'immigrazione in generale.

Dare informazione sull'immigrazione in maniera positiva da parte dei media porta ad atteggiamenti significativamente più positivi rispetto all'immigrazione e agli immigrati.

Visti i presupposti illustrati sopra sarebbe opportuno implementare azioni di Educazione degli adulti a doppio senso, per gli autoctoni e per gli immigrati, con l'obiettivo principale di intervenire nella diffusione del discorso sulla migrazione, concentrandosi anche sulle narrative che sono uno strumento privilegiato per riuscire a comprendere il ruolo e la responsabilità di ciascun stakeholder nel rapporto tra migrazione ed emarginazione, tra migrazioni e competenze, tra migrazioni e conoscenza e infine mettere a punto azioni finalizzate al cambiamento dei meccanismi di narrazione che alimentano la distorsione della percezione.

\subsection{Linguistica Educativa e Lifelong learning}

Il concetto di Educazione degli adulti si intreccia e si sovrappone con quello di Educazione continua: la risoluzione del Consiglio sull'apprendimento permanente (Commissione delle Comunità Europee 2000) definisce e sottolinea l'importanza della formazione degli adulti all'interno di un apprendimento permanente come possibilità offerta per garantire le condizioni per l'accesso universale alla formazione, a prescindere dall'età e dallo status: comprende quindi anche i migranti.

L'apprendimento permanente, o Lifelong learning, non restringe i propri obiettivi e le proprie azioni alla formazione professionale ma mira al più ampio obiettivo di permettere un potenziamento continuo delle conoscenze degli individui che ostacoli il loro isolamento (per questioni di età o di provenienza) e che al contempo permetta il 
riconoscimento di competenze acquisite in altri contesti e in percorsi formativi sia formali sia informali.

Tuttavia, poiché non vi sono al momento documenti comunitari condivisi tra gli Stati membri dell'UE in materia di Educazione degli adulti ed esistono pochi strumenti che regolano le politiche nazionali di apprendimento degli adulti, la maggior parte degli Stati adotta proprie deliberazioni con il comune denominatore di mirare al miglioramento dell'inclusione e delle competenze di base e professionali, alla parità di accesso all'istruzione, a non discriminare gli individui in base al genere, all'età e alla nazionalità.

Si adotta in questa sede una definizione di Educazione degli adulti come varietà di forme di apprendimento intraprese dopo aver completato il ciclo iniziale di istruzione e formazione, indipendentemente dalla sua durata e dalle sue forme di realizzazione (formali e non formali), che mira ad essere permanente e a uno sviluppo della società della conoscenza, delle pari opportunità, al miglioramento dei livelli di vita e di lavoro, alla cittadinanza attiva.

L'obiettivo di creare modelli innovativi di educazione alla corretta interpretazione delle narrative e la concretizzazione di essi nella sperimentazione di azioni che favoriscano il contatto sia tra migrante e non migrante, sia nei Paesi di origine che in quelli di accoglienza ma anche per diffondere la cultura della contro-narrazione creando delle strutture comunicative sia fisiche (ad es. job fairs, giornate di incontri, festival ecc.) sia virtuali (ad es. piattaforme social, siti web, mostre itineranti ecc.), nonché materiali didattici e di divulgazione è divenuto di urgente implementazione e realizzazione.

È ormai comunemente riconosciuto che l'integrazione economica è un tassello fondamentale per l'integrazione dei migranti ma anche che può essere ottenuta grazie all'istruzione, condizione quest'ultima indispensabile perché si realizzino tutte le tipologie di integrazione: economica, sociale, psicologica, politica. In Europa si registra una grande varietà, per tipologia e qualità, di programmi di istruzione concepiti ed erogati specificatamente per gli immigrati anche se con alcuni aspetti comuni: non ci sono abbastanza programmi per i discenti immigrati e, in taluni casi, gli insegnanti hanno scarsa conoscenza del target in questione e su come rendere il processo di istruzione un'esperienza significativa o di come accrescere l'effetto dell'insegnamento/apprendimento.

I meccanismi che disciplinano l'ingresso e il soggiorno di cittadini stranieri nell'UE possono essere suddivisi in quattro categorie, in base al motivo della migrazione, per ciascuna categoria occorrono specifiche linee di Lifelong learning e di Educazione.

A) L'Unione Europea è particolarmente favorevole ad attrarre persone altamente qualificate che potrebbero contribuire a rafforzare la propria competitività e dunque all'immigrazione per motivi di lavoro. La creazione della Carta blu UE, un 
permesso di lavoro per cittadini altamente qualificati non comunitari offre il diritto di circolare liberamente all'interno dell'Unione, concede una serie di diritti socio-economici e facilita l'accesso al mercato del lavoro. Manca l'indicazione delle competenze in lingua settoriale che sono richieste per il conseguimento di questo obiettivo.

B) Nel 2016 è entrata in vigore la direttiva sulle condizioni di ingresso e soggiorno di cittadini di Paesi terzi per motivi di ricerca, studio e formazione, o per progetti educativi e di au pairing.

C) Il ricongiungimento familiare rimane una delle principali cause per cui i cittadini di Paesi terzi possono spostarsi in UE, che a sua volta cerca di facilitare le relative procedure, tanto che per i cittadini extracomunitari che risiedono legalmente nell'UE è abbastanza facile farsi raggiungere dalla famiglia a condizione che dimostrino di soddisfare determinati requisiti, come quelli di avere un alloggio adeguato e risorse economiche sufficienti per il mantenimento della famiglia, ma non una educazione di livello medio-elevato. L'Unione Europea si è infine dotata di una Carta dei diritti fondamentali al fine di proteggere chi è nel bisogno in accordo con la Convenzione di Ginevra (1951) relativa allo status di rifugiato. L'asilo, meccanismo che garantisce protezione a persone in fuga dalla loro casa, può essere concesso ai migranti che rivendicano lo status di rifugiato. Secondo le informazioni fornite dall'UNHCR, alla fine del 2015 erano più di 23 milioni i rifugiati in tutto il mondo (la Germania ha ottenuto il maggior numero di domande d'asilo nel mondo), dati che richiedono azioni normative a livello comunitario volte a garantire parità di livello di accoglienza in tutti gli Stati membri. Il progetto COMMIT, rilevando le lacune in questo ambito, ha proposto strumenti operativi di preparazione alla partenza e per le fasi successive all'arrivo.

Da questa breve panoramica sulle categorie e sui bisogni degli immigrati consegue che nella progettazione della metodologia formativa per l'Educazione degli adulti devono essere applicati, e condivisi, alcuni principi che riguardano i principali attori e gli approcci formativi.

\section{Motivazione}

I percorsi di apprendimento devono avere luogo in adeguati ambienti e in un clima di rispetto, fiducia e accettazione sono essenziali per il successo della formazione con qualsiasi tipo di pubblico e ancor di più con quello oggetto di queste riflessioni.

Parlare di contatto e identità culturale, di plurilinguismo e insicurezza linguistica, di educazione e formazione permanente permette di 
introdurre il ruolo essenziale che ha, e ha sempre avuto, la motivazione nel generale processo formativo e nel processo di acquisizione delle LS/L2. La motivazione è stata oggetto nel corso del tempo di approcci molti diversi: centrati su aspetti emotivi e metacognitivi, su aspetti della personalità e sugli stili di attribuzione dei significati, o ancora sugli aspetti sistemico-relazionali e sulle modalità di insegnamento. Oggi viene analizzata e potenziata in rapporto di tipi di pubblici, sulla base dei loro profili e correlata all'analisi del costrutto psicosociale degli atteggiamenti e degli spazi di apprendimento. Oltre alla motivazione estrinseca (Skinner 1974) e a quella intrinseca con adulti immigrati possono essere sviluppate anche quelle situazionale (dipendente dalle specifiche caratteristiche di eventi o contesti, cf. Hidi 1990), e di inserimento e appartenenza sociale (Urdan, Maehr 1995). Mirare soprattutto allo sviluppo dell'interesse individuale porta a una motivazione più stabile e al desiderio di incrementare le proprie competenze, a investire nel futuro e all'autodeterminazione, che comporta un atteggiamento attivo del soggetto nel proprio apprendimento e nella costruzione dei personali schemi di sé essendo anche capace di percepire la propria competenza nell'affrontare determinati compiti, giudicando le abilità possedute e valutando la propria capacità di riuscita.

Oltre alla motivazione di carattere strumentale si sa ormai che è presente anche una dimensione più intrinseca, presumibilmente condizionata da una serie di fattori sia interni sia esterni, da inquadrare nel processo globale di inclusione. Lo studio della L2 da parte di adulti immigrati è probabilmente sostenuto da diverse tipologie di motivazioni ed elementi e caratterizzato da un continuum di aspetti estrinseci e intrinseci sui quali intervengono una serie di variabili interne (età, cultura di origine, livello di scolarizzazione, percorso scolastico pregresso, stato psicologico di negazione/accettazione della vita nel Paese di accoglienza, atteggiamento emozionale negativo/positivo-passivo/reattivo) ed esterne (ambiente, etica, influenza di lingue/dialetti, interazioni comunicative, opportunità culturali) e di rappresentazioni sociali che ne alterano e alternano il grado, il livello e la specie. Per l'apprendimento gli adulti necessitano principalmente di motivazione interna. L'apprendimento dovrebbe essere significativo e rilevante, rispondendo ai loro bisogni, interessi e problemi reali. Il fattore chiave che motiva l'adulto all'apprendimento è la rilevanza dell'apprendimento stesso.

\section{Approcci formativi}

All'interno di una cornice teorico metodologica di tipo comunicativo umanistico-effettivo si dovranno, da una parte, sviluppare l'autonomia e la creatività dell'apprendente in un'atmosfera piacevole e rilassata, dall'altra, stimolare le capacità cognitive sviluppate dagli adulti 
presentando però obiettivi chiari e semplici e utilizzando testi legati alle loro esperienze e ai vissuti quotidiani. ${ }^{4}$

Dal momento che le finalità individuate sopra impongono, da una parte, uno studio degli aspetti linguistici che vada al di là di quello formale e astratto delle lingue e, dall'altra, il raggiungimento di una competenza socio e interculturale che permetta di divenire cittadini del mondo (superando l'intolleranza e le incomprensioni xenofobe), assumono fondamentale importanza il concetto di interazione - fra culture, fra apprendenti, fra visioni del mondo - e la valorizzazione della diversità. Conseguentemente sarà importante perseguire l'acquisizione non di una semplice e definita padronanza linguistica o di singoli contenuti disciplinari, isolati l'uno dall'altro, con l'abilità del nativo come modello ideale, ma di un repertorio linguistico e mentale nel quale tutte le conoscenze e le esperienze in fatto di lingua/e e cultura/e, per quanto parziali possano essere, abbiano un loro ruolo e interagiscano fra di loro, contribuendo a rendere effettiva la comunicazione. Poiché gli aspetti linguistici e culturali fanno entrambi parte della competenza comunicativa in quanto capacità di interpretare e di usare in maniera appropriata le norme sociali che governano i comportamenti nelle varie situazioni - cioè «competenza comunicativa interculturale» (Balboni 2007, 16) - dovranno essere oggetto di attenzione sia la comunicazione quotidiana che quella microlinguistica (delle singole materie).

Ciascun individuo ha la propria forma di apprendimento a seconda del canale di percezione preferito - visivo, auditivo o cinestetico - e dunque la didattica prevederà classi ad abilità miste e differenziate al loro interno in base ai livelli presenti nel gruppo e alle predisposizioni degli individui; attività con input comprensibili e che stimolino la riflessione nel rispetto delle forme di apprendimento preferite dagli apprendenti e con adeguate tecniche didattiche.

Dato ormai per assodato che il linguaggio non può comprendersi al di fuori di un contesto di cui fa parte dandogli l'esistenza e facendone un'esperienza (Ochs 2012) ne consegue che la lingua influisce sull'esperienza umana, non è fine a sé stessa: dunque qualsiasi forma di educazione non può prescindere dalla conoscenza della/e lingua/e oltre che del quadro culturale e del senso di una data interazione in un dominio preciso, e con attori determinati.

La riflessione sulla sinergia di lingue e linguaggi conduce verso il concetto di multimodalità, aspetto importante scaturito dallo sviluppo delle tecnologie dell'informazione e della comunicazione (TIC), che si fonda sulle possibilità offerte dai gesti, dai suoni, dal visivo e da al-

4 Si vedano le scelte metodologiche suggerite e adottate per uno specifico tipo di pubblico adulto, gli stranieri reclusi, in cui si è anche giunti a invertire il consueto ordine di priorità di abilità scritte e orali per i livelli pre-A e A1/A2 (Benucci 2007a; Benucci et al. 2010; Benucci, Grosso 2012). 
tri simboli semiotici compreso, ma senza limitarsi ad esso, il linguaggio, in quanto tutti facenti parte della comunicazione: nell'approccio multimodale dell'educazione, la comunicazione non è possibile con il solo mezzo del linguaggio ed è vista come un processo in continuo movimento, fluido, variabile a seconda delle lingue, delle culture e dei contesti coinvolti. Questa ultima considerazione è di particolare importanza per l'educazione di adulti immigrati, come si vedrà nel prossimo paragrafo dedicato alle risorse per l'apprendimento e alle modalità della conduzione delle lezioni.

\section{Apprendenti}

La formazione dovrebbe basarsi sulle esperienze degli apprendenti e incoraggiarli ad una partecipazione attiva (apprendimento esperienziale) partendo dalla considerazione che l'esperienza è una risorsa per l'apprendimento. Gli adulti, indipendentemente dal loro livello di istruzione, dispongono di una certa quantità di esperienze e conoscenze antecedenti che devono essere a fondamento per i nuovi apprendimenti, aspetto ormai dimostrato dalle neuroscienze. In una didattica di tipo costruttivista l'apprendente deve potersi sentire a proprio agio e libero di prendere iniziative ed esprimere sé stesso, in autonomia, pur se guidato. L'adulto ha bisogno di un controllo sul processo di apprendimento e di un feedback da parte del docente, non però di tipo punitivo: gli errori commessi sono visti ed utilizzati come un supporto al miglioramento e non come un fallimento. Questo tipo di approccio richiede responsabilità, uno sforzo di concentrazione e un impegno che senza adeguata motivazione e risultati tangibili possono indurre ad abbandonare il patto formativo.

\section{Docenti}

Il formatore sarà un facilitatore e una guida all'insegnamento. Deve mirare al mantenimento dell'identità culturale degli immigrati (e dei loro figli) e a valorizzare la potenziale ricchezza formativa insita nella diversità delle classi. Pur con la finalità prevalente di far conoscere la lingua e la cultura del Paese di arrivo devono essere previste azioni e iniziative bilingui e biculturali vs. plurilingui e pluriculturali, poiché la formula bi- e plurilinguistica / culturale è quella più idonea alla futura politica culturale, sia perché accanto alla finalità del mantenimento dell'identità culturale in cittadini di quel dato Paese va unita quella di formare cittadini del mondo, sia perché non si può non tenere conto della ricchezza che offre la particolare situazione di tali classi che permettono di unire due o più modelli scolastici, approcci alla cultura, allo studio, alla lingua ecc. 
In tale situazione di insegnamento, anche se non solo in questa, il docente è essenzialmente un mediatore culturale, dunque prima di iniziare a svolgere la sua attività dovrà porsi almeno una serie di domande che gli serviranno per condurre un insegnamento di tipo contrastivo-comparativo. Le prime sono di tipo linguistico, le seconde di genere culturale, intendendo per cultura una concezione più vasta di quella tradizionale scolastica, nozionistica e prevalentemente umanistica (Benucci 2001).

Egli deve farsi accettare prima come persona e poi come docente e tenere conto del fatto che ogni suo atto comunicativo genera almeno due tipi di comportamento: condiziona o determina i comportamenti immediati, concreti ed individuali; modella l'assetto cognitivo e le norme generali di comportamento che ne derivano tramite l'instaurazione di habitus o modelli sociali e culturali. Deve predisporre le condizioni perché si instauri una mediazione interculturale rispetto ai punti di vista (valori, opinioni, mentalità, credenze), agli stili di vita (riti, costumi, bisogni), ai desideri (progetti, aspettative), ai sentimenti (tensioni affettive, stati d'animo, emozioni). Deve creare territori intermedi perché divengano sede di prestiti reciproci rispetto ai modi di pensare e di comunicare tenendo conto che accanto agli 'universali' della mente (ordinare, risolvere problemi ecc.), strettamente interrelati a quelli del linguaggio (descrivere, argomentare, discutere ecc.), anche le manifestazioni emozionali (ansia, paura, soddisfazione ecc.) costituiscono l'oggetto pedagogico peculiare della mediazione interculturale.

\section{Educazione degli adulti, Linguistica Educativa ed Educazione Linguistica}

Non si può parlare di Educazione agli adulti migranti senza accennare al campo di interesse della Linguistica Educativa che, come ricordava Ferreri (2002), analizza e studia i fattori che favoriscono l'apprendimento o che invece possono rallentarlo, individuando i rapporti tra lingue nella mente dell'apprendente, in particolare per quanto riguarda gli aspetti psicocognitivi, e si occupa della componente semiotica dell'apprendimento assegnando un ruolo al contesto situazionale e al contesto sociale.

Nella Linguistica Educativa, come nell'Educazione Linguistica (EL), si impone il primato del significato e insieme del senso condiviso che rinviano alla socialità e correlano l'individuo alle comunità di cui ogni lingua è espressione. Dunque, la / le teorie devono permettere di rispondere ai seguenti assunti:

1. l'apprendimento di una lingua non può essere conseguito in isolamento e a prescindere dai soggetti, da qui l'esigenza di una considerazione complessiva delle conoscenze e pratiche 
linguistiche e di un insegnamento tarato sulle conoscenze reali degli apprendenti;

2. l'apprendente non può essere lasciato solo nella costruzione del suo apprendimento ma va posto in un ambiente sociale, entro cui ogni componente compie lo sforzo, condivide il peso e partecipa all'operazione di co-costruzione dei significati da acquisire nella lingua target.

Il modello cui tende la Linguistica Educativa deve spiegare la relazione tra l'apprendente e il suo ambiente, come esito di una ecologia di sistema. Lier si pone come il promotore di una linguistica educativa ecologica.

Ecological linguistics (EL) focuses on language as relations between people and the world, and on language learning as ways of relating more effectively to people and the world. The crucial concept is that of affordance, which means a relationship between an organism (a learner, in your case) and the environment, that signals an opportunity for or inhibition of action. (Lier 2004, 5)

L'Educazione Linguistica è quindi intesa come un asse educativo trasversale che coinvolge tutte le discipline, e non va identificata riduttivamente con il solo insegnamento della lingua target o con la sola attenzione alle lingue verbali: questa accezione ampia di EL comprende infatti anche i linguaggi non verbali (sonori, visivi, dei segni, del corpo, i linguaggi artificiali ecc.), cioè tutti i codici semiologici che la facoltà di linguaggio permette di acquisire/apprendere o costruire. E presuppone che la facoltà di linguaggio si potenzi se viene esercitata mediante l'esposizione/manipolazione e l'uso di lingue e linguaggi differenti, che favorisca lo sviluppo cognitivo e dell'intelligenza, e che si sviluppi maggiormente con l'abitudine a passare con flessibilità da un linguaggio e da una lingua all'altra (si vedano a tal proposito le teorie dell'intercomprensione e le politiche sul plurilinguismo).

\subsection{Didattica per soggetti svantaggiati}

Gli insegnanti di lingua, i mediatori e i tutor hanno una influenza sociale e politica dato che sono i principali attori dell'adattamento al cambiamento e della gestione della diversità sociale di oggi; essi possono giocare un ruolo non secondario per integrare la diversità, per favorire la collaborazione tra differenti apprendenti attraverso il lavoro di gruppo e la gestione di processi di apprendimento, valorizzando la diversità e il plurilinguismo.

Innanzi tutto è bene chiarire che per soggetti svantaggiati si intendono e si considerano in questa sede gli immigrati adulti con scarse 
o nulle competenze linguistiche in L2, che devono affrontare percorsi di inserimento nel Paese di arrivo, donne immigrate, alunni della scuola dell'obbligo con competenze in L2 insufficienti per ottenere un profitto scolastico, detenuti stranieri. Non ci si occupa in questa sede di BES (Bisogni Educativi Speciali).

Per tutte queste categorie sono vari, per tipologia e qualità, i programmi di istruzione avviati nei diversi Paesi dell'Europa che, come già accennato, hanno però il comune denominatore di essere insufficienti a dare risposta alle esigenze e alle motivazioni degli apprendenti ma soprattutto di essere spesso erogati in seno a progetti specifici di formazione-ricerca, mentre manca per lo più una politica educativa in questo ambito che sia condivisa e messa a sistema in tutta l'Europa.

Gli insegnanti di L2 sono figure professionali alle quali spesso sono richieste doti camaleontiche per poter offrire il loro contributo a situazioni di insegnamento che oggi sono ormai molto variegate, ampie e complesse, e che vanno dall'insegnamento tradizionale a studenti il cui interesse principale è 'il viaggio di stendhaliana memoria', a quello di bambini giunti con i loro genitori in cerca di situazioni di vita migliori, ai rifugiati o ai detenuti stranieri sempre più numerosi nelle carceri europee. La formazione loro offerta si limita per lo più ai contenuti disciplinari e poco affronta i problemi psicologici e sociologici, metodologici specifici per pubblici con problematiche.

Qualsiasi strategia didattica obbliga invece a prendere atto delle diversità di ciascuno e a selezionare modalità differenziate di intervento in base a fattori sia affettivi e sociali (variabili motivazionali e attitudinali, fattori attinenti la personalità, dinamiche del gruppo ecc.) sia cognitivi (capacità intellettive, attitudini evolutive ecc.). Dunque la programmazione di un intervento didattico strutturato comporta: momenti conoscitivi (dove e su chi si interviene: il Paese, lo studente, i bisogni e le motivazioni), momenti di decisione (in base ai traguardi che vengono posti: lingua e cultura coinvolte, programmi ufficiali) e momenti operativi (come intervenire: materiali didattici e tecniche didattiche), clima della classe e sua gestione.

Nel modello costruttivista l'apprendente è al centro del processo di apprendimento come agente attivo e quasi autonomo della propria conoscenza con tutto il suo bagaglio di esperienze fisiche, mentali e sociali. Gli insegnanti ideali divengono così dei facilitatori, aiutano gli studenti nella costruzione dei sapere a scapito delle mere conoscenze di nozioni, li incoraggiano ad analizzare e a valutare idee e schemi personali e del gruppo classe in un ambiente di apprendimento collaborativo, li aiutano a sviluppare il pensiero critico e la consapevolezza, sommando nuove e vecchie conoscenze.

La riflessione è centrale nell'approccio costruttivista rivolto ad adulti, portatori di esperienze pregresse e di aspettative, con abilità metacognitive già sviluppate e necessità di monitorare e valuta- 
re i propri progressi ma non sempre essi sono abituati a esplicitarla e sfruttarla per sviluppare strategie di apprendimento efficaci. Qui risiede il ruolo fondamentale del docente /mediatore che attraverso adeguati stimoli e un clima collaborativo può offrire l'opportunità di suscitare interesse e capacità di costruzione di conoscenza da parte dell'apprendente e lo sviluppo di una vera e propria mediazione interculturale. A questo scopo sono molti utili strumenti come le griglie di autoriflessione, progetti didattici (project work) e i portfoli.

In secondo luogo gli adulti immigrati devono essere inquadrati in un generale ambito di apprendimento permanente in quanto modalità di educazione con obiettivi concreti, ben definibili e comprensibili, in continuo rapporto con le conoscenze individuali pregresse, modalità rivolta al superamento del disagio ma centrata sulla partecipazione, la condivisione e il coinvolgimento attivo, il rispetto delle identità e l'alimentazione della motivazione, con pratiche formali e informali. Non si tratta di adottare un approccio specifico quanto di partire dalla conoscenza e dall'analisi degli elementi che costituiscono barriere sociali, psicologiche, fisiche e adottare pratiche idonee al superamento di tali barriere. ${ }^{5}$

Le principali problematiche per l'insegnamento della L2 in situazioni scolastiche ed extrascolastiche, oltre a quella della selezione del materiale didattico - in quanto mezzo con il quale si attua l'insegnamento, la cui scelta e le modalità di impiego sono tutt'altro che neutre perché in quanto input la sua qualità condiziona fortemente l'apprendimento -, riguardano la gestione di livelli linguistici diversi anche all'interno della sola L1 degli apprendenti, all'interno della stessa classe, tra persone di differenti età con preparazione linguistica e livelli di competenze in L2 diversificati (A1-B2) ecc. Si cercherà di suggerire alcune modalità per affrontare queste situazioni, quali strategie adottare per classi ad abilità differenziate e per creare (facilmente) attività plurilivello dato che la maggior parte dei programmi e dei materiali didattici in circolazione non le prevedono. Le classi ad abilità miste e la gestione della diversità pongono non poche problematiche al docente, non ultima quella di gestire il tempo della classe pur frazionandolo per lo svolgimento di differenti attività in contemporanea.

Le classi di L2 plurilingue e multietniche sono composte da studenti di diversi livelli della lingua target, e conoscenze culturali caratterizzate da disequilibrio con conseguente difficoltà per l'organizzazione e la gestione della didattica, anche solo per la comunicazione

5 Sono molti ormai i progetti rivolti a 'soggetti svantaggiati', se ne citano in questa sede solo alcuni a scopo esemplificativo: Teprom (Repubblica Ceca) per soggetti in cerca di lavoro, Young in Prison (Svezia) rivolto a ex detenuti, Melete (Austria) rivolto a migranti, SPRAR / SIPROIMI (Italia) per richiedenti asilo e rifugiati, Mobil (Germania) per l'educazione di prossimità. 
docente-studente. ${ }^{6}$ In queste classi la lezione frontale tradizionale si è ormai da tempo dimostrata inefficace. Le classi ad abilità linguistiche differenziate (o meglio CAD) devono essere gestite con un modo di osservare la realtà della classe che tiene in considerazione la natura e il tipo di apprendimento di ogni individuo, che pone attenzione - e valorizza - la differenza di ogni apprendente: costituiscono un sistema dinamico dipendente dal tipo e dalla natura dell'apporto di ciascun individuo. Come già detto, gli apprendenti differiscono tra di loro per caratteristiche individuali quali il livello linguistico, il background culturale, l'attitudine verso le/a lingue/a, gli stili cognitivi e di apprendimento, la L1, la conoscenza del mondo e di altre lingue, il repertorio linguistico, l'età, l'esperienza di apprendimento, ma anche il genere, la motivazione, gli interessi, i bisogni e la capacità/volontà di autopromozione. Dunque fattori di differenza: personali, socioculturali, relazionali. ${ }^{7}$

I fattori personali variano in base a: bisogni e motivazioni di studio (motivazione estrinseca/intrinseca ecc.), stili cognitivi (approccio preferito nell'apprendimento - sottolineare, glossare, riassumere, prendere appunti ecc. -, nella rappresentazione della realtà ecc.), stili di apprendimento (tendenza a preferire un determinato modo di apprendere/studiare), attitudine all'apprendimento linguistico (capacità di riprodurre correttamente suoni, tipo di memoria prevalente ecc.), tipi di personalità (introversa/estroversa, autonoma/dipendente), tipi di intelligenza (multipla, linguistica, logico-matematica ecc,), età e patologie (capacità di memorizzazione, problemi di udito ecc.).

I fattori socioculturali riguardano l'ambiente familiare (le risorse parentali, la vicinanza, la lontananza, il 'lessico familiare' ecc.), l'ambiente sociale (classi sociali di appartenenza, distanza tra il Paese ospitante/ di destinazione e il Paese di provenienza/studio, i servizi a cui si accede, gli ambiti situazionali e i domini di interazioni più frequenti ecc.), la storia migratoria (integrazione vs. isolamento, vulnerabilità psicologica ecc.), l'emancipazione o il fallimento (il modo di pensare, i pregiudizi, il condizionamento, la libertà, la privazione della libertà ecc.).

Fattori relazionali da analizzare sono il clima della classe, il tipo di relazione che si instaura tra docente e studenti, l'inclusione o l'esclusione di individui da parte del gruppo classe, la possibilità offerta di costruzione della conoscenza attraverso il dialogo e la relazione con gli altri, il clima di collaborazione e cooperazione, la tipologia di compiti proposti e preferiti (compiti autentici, situati, motivanti e vicini al vissuto degli apprendenti).

6 Molti studi indicano le caratteristiche e le differenze degli apprendenti (per una prima bibliografia di riferimento cf. Ur 1996; Tice 1997, Şalli-Çopur 2005; Caon 2008a) tuttavia pochi offrono possibili soluzioni operative per la gestione da parte dell'insegnante.

$7 \mathrm{Su}$ questi aspetti si veda anche il capitolo 6 di questo volume, in particolare $\S 6.4$. 
Le CAD richiedono dunque al docente di cambiare il proprio modo di osservare la realtà della classe perché sono un sistema dinamico dipendente dal tipo e dalla natura dell'apporto di ciascun individuo: gli interessi degli apprendenti cambiano in base all'età e all'interno dello stesso periodo di apprendimento; modalità e livello di partecipazione variano da soggetto a soggetto, soprattutto a livello di produzione orale che può essere differente in base all'età, alla conoscenza della L2, agli interessi, alla capacità di lavorare in gruppi, in coppia ecc.; il livello di apprendimento degli studenti è influenzato da ritmi più o meno veloci e dalle caratteristiche individuali così come dalle attività preferite e dalle dinamiche relazionali della classe. In sintesi, il docente deve essere in grado di gestire una molteplicità e varietà di contenuti, materiali e procedure didattiche, di ricorrere a una metodologia didattica variata, organizzare flessibilmente la classe, stimolare la cooperazione, predisporre esercizi e attività di diversa tipologia e che tengano conto della velocità di esecuzione, costruirsi o modificare i materiali didattici perché i manuali solitamente vengono pensati per classi omogenee senza differenziare compiti o argomenti. Deve poi evitare conflitti, incomprensioni, pregiudizi.

Lo stile di insegnamento incide a livello cognitivo, affettivo (motivazione), relazionale (modelli comportamentali), emotivo (stress negativo), culturale (aspettative e valorizzazione). Fattori del docente che incidono sullo stile di insegnamento sono le convinzioni formate nella carriera di studente (poi di docente) in base alle esperienze pregresse, l'influenza di ambienti socioculturali in cui ha studiato e insegnato in precedenza, il tipo di formazione glottodidattica, lo stile comunicativo, l'immagine che ha del suo ruolo (cioè l'autovalutazione del proprio modo di insegnare). Il clima della classe è dettato dalle azioni che vi avvengono e dalla loro qualità: nella didattica cooperativa accanto allo sviluppo di competenze linguistico-comunicative, metacognitive, metaemotive e interculturali va curato quello di competenze sociali e relazionali. Non è affatto facile gestire tutte queste variabili.

Gli insegnanti sono chiamati dunque a: reimpostare le modalità di interazione e gestione della classe; fornire istruzioni chiare (e con lessico di base), ripetitive; gestire la formazione di gruppi e saper stimolare la partecipazione attiva di tutti i componenti; saper alternare momenti di gestione di tutta la classe e di recupero di soggetti meno reattivi e competenti, anche con svolgimento di attività con differenti livelli di difficoltà in contemporanea; saper individuare il livello medio di performance e fornire un feedback individuale; organizzare un apprendimento basato su compiti concreti e reimpiegabili nella vita quotidiana e/o nel tirocinio, oltre ovviamente a insegnare a leggere, a selezionare le informazioni, a studiare, ad essere autonomi. E ancora l'insegnante e il mediatore deve imparare ad autovalutare il proprio comportamento e chiedersi: come mi rivolgo agli 
studenti, come li gratifico, come li coinvolgo, quali modalità di lavoro privilegio (a coppia, in gruppo ecc.), quali modalità di correzione degli errori impiego, con quali modalità stimolo la motivazione, quali tecniche didattiche uso di preferenza, a quali tecnologie didattiche ricorro più spesso, come organizzo la disposizione della classe e la mia posizione, quali modalità di interazione con gli studenti preferisco ecc. Gli apprendenti a loro volta dovrebbero imparare ad assumere sfide, acquisire competenze testuali nella loro globalità, imparare a non bloccarsi se ci sono parole o frasi che non capiscono, cercare di ridurre il filtro affettivo, abituarsi ad imparare a riflettere su ciò che stanno facendo, imparare a cercare il feedback degli altri, saper combinare elementi linguistici e paralinguistici diversi, apprendere ad autovalutarsi e autopromuoversi.

La diversità degli attori del processo di insegnamento/apprendimento e delle variabili fino a qui elencate, che sembrano un problema insormontabile, possono tuttavia divenire una opportunità di sviluppo significativo di competenze. Più che di diversità si parla però oggi di 'superdiversità' rivolgendo l'attenzione più al codice della comunicazione che alle altre componenti: la superdiversità è caratterizzata da un consistente ricorso al code-switching e al code-mixing in cui il linguaggio e le lingue sono considerati dagli stessi parlanti strumenti di potere, di (re)inserimento, interazione, successo scolastico e/o professionale, senza eccessive preoccupazioni per la correttezza dei messaggi e la forma utilizzata nella L2.

Strategie didattiche per insegnare una lingua straniera in una classe plurilivello per un apprendimento non più indifferenziato, ma come insieme di competenze di vari livelli, per varie situazioni e per differenti parlanti (tra i quali anche i pubblici 'svantaggiati'), sono finalizzate a gestire la (super)diversità multilingue adottando una nuova concezione del locutore (Beacco 2016, 56); se la nozione di repertorio linguistico presuppone che ciascun locutore sia almeno potenzialmente plurilingue, è comunque noto che pochi trasformino questa possibilità in realtà; i percorsi formativi dovrebbero invece sviluppare la competenza plurilingue al pari delle capacità fisiche, cognitive, creative.

\section{Tecniche e materiali didattici per la gestione della diversità}

Sono da prediligere attività che coinvolgano tutti i sensi, stili cognitivi e tipi di intelligenza, motivanti, su materiali che variano per tipologia di testo, contenuti, argomenti; testi autentici; attività extra per coloro che terminano il compito prima degli altri e semplificate per chi ha maggiori difficoltà; cooperative e di gruppo assegnando compiti differenziati in base ai livelli di competenza in L2 e agli interessi; con soluzioni aperte (scrittura di una mail, completamento di una racconto scritto 
o orale o meglio di un breve filmato) perché permettono di esprimere e di potenziare la creatività; di carattere ludico (giochi, role play); che sviluppino tutte le abilità e si prestino a variare le tecniche didattiche.

Vi sono alcune modalità di differenziazione dei compiti: differenziazione parziale nella classe multilivello, consistente nella differenziazione del compito tra alunni che lavorano nello stesso giorno ad un unico tema con tre fasi: 1) lavoro sullo stesso contenuto con attività differenziate in base ai livelli di competenza; 2) differenziazione del compito in gruppi omogenei per livello; 3) restituzione in plenaria di ogni gruppo. Inoltre è possibile differenziazione per ambiti variando contenuti, obiettivi, supporti linguistici ed extralinguistici, dispositivi multimediali e tecniche; differenziare su parametri opposti - proporre attività scritte o orali (a seconda del tipo di studente prediligere attività scritte per i più bravi e orali per i meno bravi, ma questo non vale con tutti i tipi di pubblico come ad es. per gli immigrati e detenuti stranieri); permettere anche l'impiego della L1 o di un'altra lingua conosciuta; compiti diversi per qualità e quantità delle attività proposte.

Tecniche didattiche più utili per differenziare i compiti sono quelle di drammatizzazione e story-telling perché permettono di variare le attività: per esempio dalla drammatizzazione guidata, a quella libera, dalla descrizione di una storia al suo racconto soggettivo, dalla rielaborazione su input al completamento.

Esempi di differenziazione di obiettivi partendo da uno stesso testo input sono: «Leggi e cerca il significato globale», «Leggi e rispondi alle domande», «Leggi e indica se è vero o falso», «Leggi, cerca tutti i connettivi, sottolineali e cerca la loro funzione», «Leggi e dividi per blocchi», «Leggi e traduci nella tua lingua» ecc.

La stratificazione del compito è utile per la gestione delle CAD e si ottiene con tecniche e schede di valutazione stratificate dalle più semplici alle più complesse, per esempio un testo e schede con domande di comprensione graduate (le prime più semplici, generali e di comprensione globale, le successive sempre più difficili, specifiche e di comprensione dei dettagli): ogni studente sarà in grado di rispondere ad un certo numero di domande e anche di autovalutare la propria performance. Ma come si determina la complessità del compito? La griglia eleborata da Coonan (2020), che si riporta qui sotto, è una utile guida: 


\begin{tabular}{ll}
\hline Meno complesso & Più complesso \\
\hline Contesto presente & Contesto ridotto/assente \\
Testo breve & Testo lungo \\
Argomento familiare & Argomento poco/non familiare \\
Argomento poco complesso & Argomento molto complesso \\
Sintassi facile & Sintassi complessa \\
Ampia ridondanza & Poca ridondanza \\
Struttura del testo chiaramente segnalata & Struttura del testo poco segnalata \\
Lessico di alta frequenza; specifico & Lessico di bassa frequenza; generale \\
Sufficiente tempo a disposizione per l'attività & Tempo insufficiente per svolgere l'attività \\
Sequenza temporale & Sequenza non-temporale \\
Testo descrittivo, regolativo, narrativo & Testo argomentativo, d'opinione, di spiegazione \\
Un solo parlante & Più parlanti \\
L'attività consta di una sola parte & L'attività consta di più parti \\
Orientato sulla fluency & Orientato sull'accuratezza \\
Densità: pochi fatti, eventi, personaggi & Densità: molti fatti, eventi, personaggi \\
Informazione esplicit & Informazinoe implicita \\
\hline
\end{tabular}

Le attività multilivello sviluppano inoltre il pensiero 'divergente' perché non prevedono un'unica risposta corretta e possono essere svolte a diversi livelli di competenza linguistica, applicando svariate strategie, non necessariamente solo linguistiche. Su un testo come quello proposto [fig. 1] qui di seguito è possibile chiedere agli apprendenti di: leggere ed evincere le difficoltà che Anastasia dichiara di avere, leggere e trovare gli errori, leggere e confrontare con la propria situazione in L1 o in altre lingue conosciute, leggere e riassumere, leggere e ricavare una tabella. 


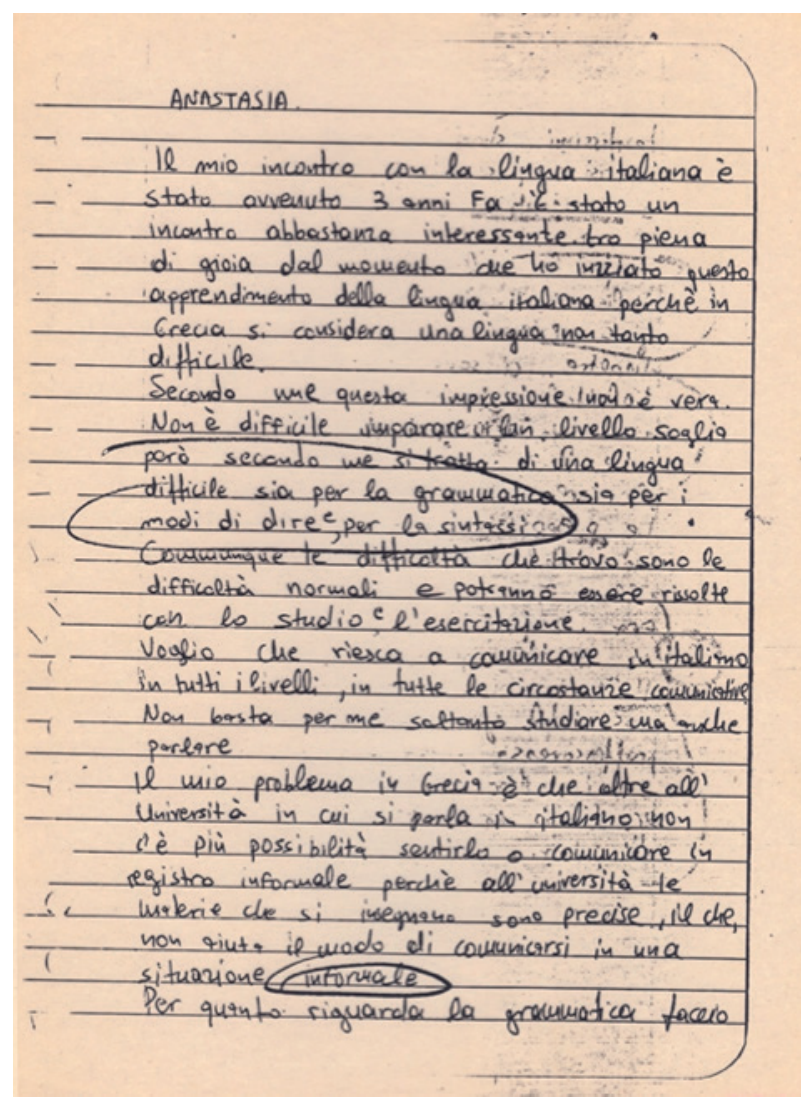

Figura 1 Autovalutazione di competenze in lingua italiana da parte di un'apprendente (corpus Analisi degli errori Benucci, non pubblicato)

La riflessione grammaticale può utilmente essere stimolata adottando l'ottica ormai sperimentata con successo nell'approccio intercomprensivo ${ }^{8}$ stimolando la costruzione di confronti tra le lingue conosciute per facilitare la costruzione di una grammatica individuale (e dunque personalizzata ed esperenziale) presentando griglie (Griglia 1) che invitano al confronto e a ricercare similitudini, magari seguite da schede di autoriflessione (Scheda 1, riadatta da Murillo, Harmegnies 2005, 89-115):

8 L'intercomprensione è un ambito di ricerca sviluppatosi dai primi anni Novanta del secolo scorso producendo un approccio e materiali didattici oltre che linee guida per la formazione di insegnanti e alunni. Per maggiori informazioni, all'interno della vasta bibliografia di riferimento, si vedano Benucci 2005; Caddéo, Jamet 2013; Capucho et al. 2007; De Carlo 2011; Meißner et al. 2011. 


\section{Griglia 1}

\begin{tabular}{|c|c|c|c|c|}
\hline it. & fr. & sp. & port. & cat. \\
\hline "amare" & "aimer" & "amar" & "amar" & "amar" \\
\hline "corale" & "chorale" & "coral" & "coral" & "coral" \\
\hline "cantare" & "chanter" & "cantar" & "cantar" & "cantar" \\
\hline "pilieri" & "pilier" & "pilar" & "pilar" & "pilar" \\
\hline "perdere" & "perdre" & "perder" & "perder" & "perdre" \\
\hline & \multicolumn{4}{|c|}{$\begin{array}{l}\text { Spesso le vocali iniziali sono identiche, completa (con francese, } \\
\text { spagnolo, } \\
\text { portoghese, catalano): } \\
\text { - "a ": it. amico, sp. amigo fr. ami...; } \\
\text { - "e": it. esercizio, cat. exercici, port. exercicio, ...; } \\
\text { - "i": it. ignorare, isole, port. ignorar, ilha...; } \\
\text { - "o": it. obbedire, fr. obéir, sp. obedecer,...; } \\
\text { - "u ": it. ùtile, cat. util, port. util,.... }\end{array}$} \\
\hline
\end{tabular}

\section{Scheda 1}

Mi aiuto con la mia lingua madre perché è molto simile alla lingua $X$.

Conosco altre lingue romanze/germaniche/slave ecc. e quindi comprendo meglio perché simili alla lingua $X$.

Quando ho difficoltà a capire quel che sento/leggo, rinuncio e smetto di ascoltare/

leggere.

Trovo che ascoltare/leggere in lingua $\mathrm{X}$ sia più difficile che leggere, parlare o scrivere in lingua $\mathrm{Y}, \mathrm{W}$ ecc.

Per me comprendere la lingua $X$ mentre ascolto/leggo è una sfida.

Ascoltare/leggere la lingua $\mathrm{X}$ non mi innervosisce.

Prima di cominciare ad ascoltare, faccio un piano su come ascolterò.

Prima di iniziare a leggere scorro tutto il testo e faccio ipotesi.

Mi concentro di più sul testo quando ho difficoltà a capire.

Mi concentro sul tono della voce.

Mi concentro sui gesti.

Mi concentro sugli aspetti grafici.

Utilizzo le parole che capisco per indovinare il significato delle parole che non comprendo.

Dopo avere ascoltato, ripenso a come ho ascoltato e a cosa potrei fare in modo diverso la volta successiva.

(adattato da Benucci, Cortés Velásquez 2011)

Aspetti e azioni sicuramente positivi dell'intercomprensione utili per la didattica per abilità differenziate sono: selezionare e gerarchizzare gli obiettivi di apprendimento; stimolare i soggetti a far leva sull'insieme delle loro competenze sia culturali sia linguistiche, acquisite in ambito istituzionale o personale (musica, viaggi, conoscenze ecc.); costruire i rudimenti di una grammatica della comprensione individuando i punti di convergenza translinguistica (regole di passaggio) 
e i 'tranelli' linguistici da evitare (regole di vigilanza). Attivando le competenze pregresse (non solo lessicali) si richiamano conoscenze di domini, e al loro interno di situazioni comunicative (luoghi, persone, spazi, esperienze di contatto, conoscenze specializzate, elementi paralinguistici, aspetti non verbali ecc.) e ci si abitua così progressivamente a mobilitare il proprio potenziale cognitivo.

La conoscenza degli obiettivi permette a chi studia di dirigere meglio la sua attività e il suo interesse: è provato che si impara prima e meglio se si conoscono (e capiscono) gli obiettivi del lavoro. Se un determinato contenuto è stato oggetto di varie operazioni (analizzato, riassunto, trasformato) le sue tracce in memoria saranno più profonde rispetto a quelle lasciate da un'esposizione passiva. Ė utile aver presente che le persone ricordano (Ginnis 2002) il 10\% di ciò che leggono, il $20 \%$ di ciò che sentono, il $30 \%$ di ciò che vedono, il $50 \%$ di ciò che sentono e insieme vedono, il $70 \%$ di ciò che dicono $\mathrm{e}$ il $90 \%$ di ciò che dicono e insieme fanno.

Nella gestione delle CAD la didattica è essenzialmente per task ma ricorre a tutto ciò che può far sentire a proprio agio l'apprendente adulto; sono quindi prioritarie le attività comunicative, di comprensione, di riflessione interlinguistica e interculturale ma per determinati gruppi e scopi si può ribaltare l'ordine e magari partire da quelle scritte (come per esempio con detenuti stranieri) sempre tenendo ben presenti i principi della differenziazione e della stratificazione:

- differenziazione di: approcci, tecniche, tipologia di task, ritmi e gestione della lezione, input fornito dal docente;

- stratificazione di: attività dalla più semplice alla più complessa, in contemporanea ma senza che tutti eseguano tutte le attività, di task, tecniche didattiche e contenuti.

La didattica per compiti prevede attività in cui la L2 è usata dall'apprendente per raggiungere obiettivi extralinguistici, anche molto vari, dai più semplici come per es. compilare una classifica o descrivere un'immagine, ai più complessi come per es. ricostruire la trama di un film a partire dai racconti parziali dei compagni o impostare il programma di viaggio per una gita di classe. A partire da uno stesso documento input possono essere organizzati differenti task da far svolgere a gruppi di apprendenti in base ai loro livelli di competenza ma sempre in accordo con il principio del primato del significato di modo che l'attenzione si concentri su ciò che stanno dicendo più che su come lo stanno dicendo: la forma non è primaria e si impiegano le strutture linguistiche precedentemente presentate dall'insegnante o già conosciute lasciando liberi gli apprendenti di eseguire il compito utilizzando le forme linguistiche che hanno a disposizione nel proprio repertorio (in una attività tradizionale si chiede invece che gli apprendenti riutilizzino i pattern linguistici precedentemente proposti dall'insegnante). Inoltre questo tipo di didattica è aderen- 
te alle attività del mondo reale poiché per portare a termine il task si compiono operazioni tipiche del contesto non scolastico (come per es. classificare, raccontare, negoziare, descrivere, pianificare) mentre ciò non sempre accade nelle attività tradizionali come «inserire un verbo coniugato nelle frase seguente» o «compilare il cloze con i verbi mancanti».

Altre caratteristiche della didattica per compiti, che ben si addicono agli adulti oggetto di questa riflessione, sono la contestualizzazione e l'esplicitazione prioritaria dell'obiettivo. È consigliabile chiarire sempre il contesto in cui si svolge una interazione, chi sono i parlanti e i loro scopi (per es. anche il tradizionale dettato diventa un task se la consegna è «trascrivi la ricetta della torta di mele che tua nonna ti sta illustrando nei dettagli del procedimento e che ti serve ad acquistare gli ingredienti per fare la torta per la cena di questa sera»); fare in modo che lo scopo principale dei partecipanti sia portare a termine il compito (nelle attività tradizionali è invece quello di riuscire a utilizzare correttamente la struttura linguistica) anche perché l'insegnante valuterà il risultato finale, la capacità di portare a termine il task e non la capacità di utilizzare correttamente determinate strutture linguistiche.

Attività che compaiono con maggiore frequenza nella didattica per task sono: prendere decisioni in gruppo; sostenere la propria opinione e cercare di persuadere gli altri; discutere informalmente in gruppo; cooperare con gli altri per il raggiungimento di uno scopo pratico; dividersi e organizzare il lavoro; intervistare ed essere intervistati; ricercare informazioni su Internet o in biblioteca; comprendere un testo scritto per ricavarne dati e informazioni; elaborare testi scritti di supporto a materiale iconografico; preparare una relazione scritta; elaborare dati sotto forma di grafici; esporre il proprio lavoro.

Ecco un esempio di task per osservare l'interlingua.

Descrizione di un'immagine usando immagini di complessità variabile in base al livello linguistico e cognitivo. L'immagine può essere accessibile alla vista di entrambi i partecipanti ma per rendere la situazione più stimolante dal punto di vista comunicativo sarebbe opportuno che chi ascolta non potesse vederla ricorrendo a vari stratagemmi come chiedere di tenere il libro davanti a sé, mettere uno degli alunni di spalle ecc. Chi ascolta potrebbe cercare di riprodurre l'immagine descritta disegnandola, scegliendola tra altre immagini simili. Durante la descrizione l'altro compagno (o il docente) interviene per rassicurare (si, va bene, okay, allora, ci sono due persone vicino alla casa), stimolare (e poi? cosa c'è ancora? ci sono altre cose?), chiedere chiarimenti (scusa non ho capito bene; me lo dici ancora perché non ho sentito?), scherzare (che disegno difficile, non so se sono capace!; sei bravissimo!; chissà cosa salta fuori...), non bisogna fare domande precise di contenuto, del tipo di che colore sono gli alberi? o quante persone ci sono vicino al giornalaio? Se l'ap- 
prendente non conosce le parole esatte per indicare un oggetto lo si incoraggerà a dirlo in altri modi, con una perifrasi (l'edicola può diventare una casa coi giornali), non si correggerà la sua versione, gli unici casi in cui si interverrà saranno quando ciò che dice è talmente incomprensibile da pregiudicare la comunicazione: si chiederà allora come hai detto? cosa c'è vicino alla tavola?

Si propone qui un sintetico e schematico esempio di Unità di Apprendimento task-based:

- preparazione: presentazione delle istruzioni per il compito, svolgimento di attività che consentano di richiamare elementi lessicali / morfosintattici / culturali utili alla realizzazione del compito (fase con durata variabile in base ai bisogni degli studenti);

- ciclo del task: svolgimento del compito a coppie o a piccoli gruppi (in forma orale e/o scritta) con attività di vario tipo (es. confrontare, classificare, mettere in ordine, esprimere opinioni) mentre l'insegnante passa tra i gruppi e fornisce aiuto in caso di necessità; concluso il compito, ogni gruppo si organizza per riferire agli altri l'esito del lavoro (report e portavoce);

- focalizzazione sulle strutture: l'insegnante guida gli apprendenti all'analisi di ciò che hanno prodotto durante il task, richiamando l'attenzione sugli elementi grammaticali, lessicali, pragmatici, culturali e funzionali allo svolgimento del compito. Propone attività di pratica, dapprima più guidate poi sempre più libere, per far esercitare sulle strutture del precedente lavoro di analisi (per es. completamenti, trasformazioni, produzioni guidate) sollecita riflessioni metalinguistiche, per esempio invitando gli alunni a ricostruire insieme (induttivamente) una o più regole, oppure guidandoli all'uso del libro di grammatica o ponendo loro domande su come hanno svolto la procedura didattica.

Dunque la didattica per task ha indubbi vantaggi perché gli apprendenti riflettono sulle forme dopo averle usate, o aver provato a usarle, con un'attenzione inevitabilmente maggiore di quella solitamente riservata alla presentazione di una nuova struttura; in secondo luogo, le strutture su cui si concentra la focalizzazione linguistica sono quelle per cui gli apprendenti risultano pronti dal punto di vista dell'evoluzione dell'interlingua, perché sono quelle che loro stessi hanno cercato di utilizzare durante lo svolgimento del compito; offre uno stimolo per un uso comunicativamente autentico della lingua, senza l'obbligo di impiegare determinate strutture grammaticali; spinge gli apprendenti ad utilizzare tutte le risorse linguistiche e non linguistiche a loro disposizione esattamente come avviene nella realtà in base ai contesti d'uso e rispetto agli interlocutori; infine permette un uso della lingua maggiore rispetto a quando la comunicazione avviene prevalentemente tra i numerosi studenti del gruppo classe e l'unico insegnante. 
A conclusione si riporta un adattamento della schematizzazione della didattica per progetti e per compiti tratto da Cassandro, Maffei (2007) seguito da una schematica griglia di orientamento tra livelli, generi testuali e atti comunicativi utile per implementare questa tipologia di didattica.

\begin{tabular}{|c|c|}
\hline Maggiore autonomia & Minore autonomia \\
\hline Testi input selezionati dagli apprendenti & Testi input forniti dall'insegnante \\
\hline Insegnante 'tutor' & Insegnante 'trasmettitore di conoscenza' \\
\hline Lezione workshop & Lezione monodirezionale \\
\hline $\begin{array}{l}\text { Sviluppo di competenze trasversali } \\
\text { e generali }\end{array}$ & $\begin{array}{l}\text { Sviluppo mirato di specifiche, singole } \\
\text { abilità linguistiche }\end{array}$ \\
\hline $\begin{array}{l}\text { Gestione dei tempi e dei ritmi da parte } \\
\text { degli stessi apprendenti }\end{array}$ & $\begin{array}{l}\text { Gestione dei tempi da parte } \\
\text { dell'insegnante }\end{array}$ \\
\hline $\begin{array}{l}\text { Gestione e organizzazione del lavoro } \\
\text { da parte degli stessi studenti }\end{array}$ & $\begin{array}{l}\text { Organizzazione del lavoro da parte } \\
\text { dell'insegnante }\end{array}$ \\
\hline Attività di coppia, lavori di gruppo & Attività individuali \\
\hline $\begin{array}{l}\text { Scelta di un argomento di lavoro } \\
\text { da parte degli studenti }\end{array}$ & $\begin{array}{l}\text { Scelta dell'argomento di lavoro da parte } \\
\text { dell'insegnante }\end{array}$ \\
\hline $\begin{array}{l}\text { Obiettivo finale da raggiungere } \\
\text { (oggetto da realizzare) }\end{array}$ & Esame da superare \\
\hline Uso libero di Internet durante la lezione & $\begin{array}{l}\text { Uso guidato di Internet tramite } \\
\text { indicazioni dell'insegnante }\end{array}$ \\
\hline $\begin{array}{l}\text { Possibilità di uscire fisicamente fuori } \\
\text { della classe }\end{array}$ & Aula \\
\hline Possibilità di contatti mirati con nativi & Aula \\
\hline $\begin{array}{l}\text { Possibilità di ricercare liberamente } \\
\text { in una biblioteca o mediateca }\end{array}$ & Aula \\
\hline
\end{tabular}




\begin{tabular}{|c|c|c|c|}
\hline Livello & Genere testuale & Atti comunicativi & $\begin{array}{l}\text { Scale delle attività tratte } \\
\text { da } 01 \mathrm{M} 2002,71-109\end{array}$ \\
\hline \multirow[t]{3}{*}{ B1 } & testo descrittivo & $\begin{array}{l}\text { descrivere; confrontare } \\
\text { e contrapporre }\end{array}$ & $\begin{array}{l}\text { Scala Produzione Scritta } \\
\text { (Relazione e saggi, p. 79), } \\
\text { Scala Produzione Orale } \\
\text { (Monologo articolato, } \\
\text { p. 74) }\end{array}$ \\
\hline & annuncio di lavoro & $\begin{array}{l}\text { comprendere messaggi; } \\
\text { esprimere capacità } \\
\text { di fare qualcosa }\end{array}$ & $\begin{array}{l}\text { Scala Ricezione Scritta } \\
\text { (Leggere per orientarsi, } \\
\text { p. 88) } \\
\text { Scala Produzione scritta } \\
\text { (Pianificazione, p. 81) }\end{array}$ \\
\hline & ricetta di cucina & $\begin{array}{l}\text { comprendere e dare } \\
\text { istruzioni }\end{array}$ & $\begin{array}{l}\text { Scala Ricezione Scritta } \\
\text { (Leggere istruzioni, p. 89) } \\
\text { Scala Produzione scritta } \\
\text { (Pianificazione, p. 81) }\end{array}$ \\
\hline \multirow[t]{2}{*}{ B2 } & $\begin{array}{l}\text { articolo di cronaca } \\
\text { giornalistica }\end{array}$ & $\begin{array}{l}\text { comprendere la } \\
\text { collocazione di fatti in } \\
\text { una sequenza temporale }\end{array}$ & $\begin{array}{l}\text { Scala Ricezione Scritta } \\
\text { (Leggere per informarsi } \\
\text { e argomentare, p. 89) }\end{array}$ \\
\hline & bolletta di utenza & $\begin{array}{l}\text { comprendere } \\
\text { l'architettura del testo } \\
\text { e decodificare la lingua } \\
\text { speciale }\end{array}$ & $\begin{array}{l}\text { Scala Ricezione Scritta } \\
\text { (Leggere per informarsi } \\
\text { e argomentare, p. 89) }\end{array}$ \\
\hline $\mathrm{C} 1$ & dibattito & $\begin{array}{l}\text { argomentare e sostenere } \\
\text { una posizione }\end{array}$ & $\begin{array}{l}\text { Scala Interazione orale } \\
\text { (Scambio di informazioni, } \\
\text { p. 100) }\end{array}$ \\
\hline $\mathrm{C} 1$ & fumetti & $\begin{array}{l}\text { narrare fatti di } \\
\text { invenzione; mettere } \\
\text { in sequenza eventi } \\
\text { nel tempo }\end{array}$ & $\begin{array}{l}\text { Scala Ricezione Scritta } \\
\text { (Leggere per informarsi } \\
\text { e argomentare, p. 89) }\end{array}$ \\
\hline $\mathrm{C} 2$ & dibattito & $\begin{array}{l}\text { argomentare e sostenere } \\
\text { una posizione }\end{array}$ & $\begin{array}{l}\text { Scala Interazione orale } \\
\text { (Scambio informazioni, } \\
\text { p. 100) }\end{array}$ \\
\hline
\end{tabular}




\section{Comunicazione efficace in contesti interlinguistici e interculturali}

Antonella Benucci

Università per Stranieri di Siena, Italia

Sommario 2.1 Comunicazione verbale e non verbale. - 2.2 Problematicità della comprensione.-2.3 Comunicazione interculturale.-2.4 Mentori e mediatori: la mediazione linguistico-culturale

\subsection{Comunicazione verbale e non verbale}

Il linguaggio del corpo è in parte innato e in parte dipende dai processi di socializzazione. I meccanismi dai quali scaturisce la comunicazione non verbale sono simili in tutte le culture, ma ogni cultura tende a rielaborare in maniera differente i messaggi non verbali che risultano essere, per chi ha un altro retaggio culturale, assolutamente incomprensibili o avere un significato opposto a quello che si intendeva trasmettere. Più del $90 \%$ della nostra comunicazione giornaliera è non-verbale, è un contributo enorme al linguaggio verbale dato che la comunicazione è strettamente ambivalente: ciò che viene percepito in un messaggio vocale può essere suddiviso (Mehrabian 1972) in movimenti del corpo (soprattutto espressioni facciali) per il 55\%, in aspetto vocale (volume, tono, ritmo) per il 38\% e aspetto verbale (parole) per il $7 \%$.

In una prospettiva interculturale si deve apprendere a osservare l'altro tenendo in considerazione i linguaggi verbali, paraverbali, non verbali e i valori. Essere sensibili all'intercultura significa fare 
meno sbagli, non leggere gli altri secondo le nostre regole, non stupire e non stupirsi. Invece l'errore in cui spesso si incorre è quello di pensare che i gesti siano universali. Soprattutto nelle comunicazioni con stranieri si pensa di potersela cavare a gesti senza tener presente che questi, come la lingua, le tradizioni, le festività, l'abbigliamento, i colori ecc., vanno letti all'interno di un sistema culturale ben preciso, specifico per ogni Paese.

La decodificazione è resa possibile dalla condivisione cognitiva dei diversi sistemi di comunicazione verbale e di comunicazione non verbale. Più specificamente è veicolata da una componente verbale - ciò che si dice o si scrive, la costruzione logica di frasi, l'uso di alcuni termini piuttosto che di altri -, da una componente paraverbale - il modo in cui qualcosa viene detto: tono, velocità, timbro, volume ecc. della voce che nella scrittura si realizza con l'uso della punteggiatura, capace di infondere un certo ritmo a quello che si legge - e da una componente non verbale (cinestetica) - tutto ciò che si trasmette attraverso postura, movimenti, posizione occupata nello spazio (quale zona di un ambiente si occupa, quale distanza dall'interlocutore ecc.) e gli aspetti estetici (modo di vestire o di prendersi cura della propria persona), nella comunicazione scritta questo fattore viene meno se non si ricorre ad elementi iconici di appoggio.

La comprensione corretta di un messaggio trasmesso oralmente dipende dunque solo in minima parte dalle parole usate. In gran parte la percezione dipende dalla vocalità (ritmo, intonazione, tono, volume della voce), in grandissima parte dipenderebbe invece dalla cinesica, forma di comunicazione 'non verbale' caratterizzata da movimenti del corpo. In particolare riguarda la mimica facciale (circa 7.000 espressioni), la postura e la gestualità. I fraintendimenti delle gestualità dovuti a motivi culturali possono causare imbarazzo o malintesi e incomprensioni più gravi in ambiente di lavoro o in ambiti di contatto non mediato. I comunicatori efficaci sono capaci di variare la propria comunicazione a seconda dell'interlocutore che si trovano di fronte, volendo suscitare interesse sarebbe impossibile (o quasi) riuscire a sortire l'effetto desiderato curando soltanto l'aspetto verbale, scegliendo cioè le migliori parole possibili. È quindi essenziale espandere le proprie possibilità, adottare una estensione comunicativa, ed essere consapevoli del valore degli aspetti paraverbali e non verbali dato che le persone più espressive vengono percepite in modo più positivo sia all'inizio che nel corso dell'interazione, piacciono di più e sono considerate più attraenti, catturano più facilmente l'attenzione. I segnali non verbali vanno scelti con cura perché variano in funzione delle persone alle quali sono rivolti e del tipo di relazione esistente tra chi li emette e chi li riceve a partire da status, natura della relazione, età, livello di istruzione e ruolo.

Dunque nella comunicazione gli atteggiamenti comunicano più di quanto immaginiamo e stabiliscono relazioni: la natura di una 
relazione dipende dalle sequenze di comunicazione che a volte sono causa di conflitti perché il segnale inviato da uno dei due interlocutori influenza la risposta dell'altro e viceversa (feedback). Gli scambi di comunicazione sono simmetrici ma più spesso, in ambito sociale, complementari o asimmetrici (il comportamento di un parlante completa quello dell'altro, uno dei due è in posizione up e l'altro è down).

Per decifrare il linguaggio corporeo Borg (2009) e Pease (1998) usano la «Regola delle tre C».

- complessità: i gesti si combinano in gruppi o insiemi, un gesto in sé può non rispecchiare l'atteggiamento generale, può essere un errore prendere in considerazione un singolo gesto, se una persona si gratta il naso può mentire ma se non è inserito in un contesto il gesto può non significare niente;

- coerenza: osservare la coerenza fra messaggi verbali e non verbali perché quando c'è discordanza fra messaggio orale e messaggio corporeo si fa affidamento solo sull'ultimo;

- contesto: se una persona siede a gambe incrociate, con il viso rivolto verso il basso e le braccia conserte con le mani nascoste sotto le ascelle, è un sintomo di un atteggiamento difensivo, se però si è in una notte fredda significherà soltanto che si vuole riscaldare.

Tradizionalmente siamo abituati a riflettere esplicitamente solo sulla comunicazione verbale e paraverbale: interiezioni, ripetizioni dello stesso concetto o parola, suoni non distinguibili, manifestazioni di insicurezza e di diffidenza (lunghe pause, silenzio), richieste di aiuto o di spiegazione, esitazioni ecc. I linguaggi non verbali sono molto importanti poiché siamo prima visti e guardati che ascoltati e in caso di conflitto il non verbale prevale sul verbale: l'immigrato conosce poco o per niente la lingua del Paese ospitante, si affida dunque molto al non verbale.

Se non è sempre possibile gestire le manifestazioni involontarie del corpo (sudore, tremito, rossore ecc.) lo è per le manifestazioni volontarie che si realizzano in modo differente a seconda delle diverse culture, come per esempio il sorriso (ascoltare sorridendo in Europa equivale ad esprimere accordo, comprensione, mentre in Giappone è volontà di diniego, imbarazzo), guardare l'interlocutore negli occhi per gli europei indica franchezza ma in estremo oriente e nel mondo arabo significa una sfida o una proposta erotica; tenere gli occhi abbassati in Europa manifesta disattenzione, in Giappone massima attenzione; le espressioni del viso per i popoli del sud Europa sono normali, in Oriente poco gradite. Ancora di più si complica la situazione con l'uso di braccia e mani (alcuni segni sono rischiosi come quello per $O K$ con il pollice e l'indice uniti che nei Paesi slavi richiama la sodomizzazione) o di gambe e piedi (nel mondo arabo far vedere la suola o dondolare la gamba accavallata indica disprezzo o ri- 
fiuto del ruolo dell'interlocutore); se il sudore è naturale, l'odore di sudore per gli europei equivale alla sporcizia ecc.

Tradizionalmente i linguaggi non verbali vengono suddivisi in cinesica (gestualità, movimenti del corpo, mimica facciale), prossemica (posizioni del corpo), vestemica (abbigliamento e colori), cronemica (idee diverse del tempo), aptica (messaggi espressi tramite contatto fisico). Molto studiati sono i movimenti del corpo (cenni del capo, postura, gesti delle mani, gesti illustratori, gesti simbolici, gesti indicatori dello stato emotivo, gesti regolatori, gesti di adattamento) e tra questi lo sono particolarmente i gesti che aiutano ad esprimere meglio quello che si vuol dire, specialmente quando il messaggio si presta a doppie interpretazioni: è il caso del cosiddetto futuro di dubbio come in questo esempio «Lui avrà quarant'anni», per prendere il senso di 'è probabile che lui abbia quarant'anni / dovrebbe avere sui quarant'anni' che richiede quasi necessariamente al parlante di alzare un po' le spalle o di inarcare la bocca verso il basso, in questo modo il futuro assume la modalità di dubbio o di eventualità. I gesti fanno risparmiare tempo perché una frase pronunciata - per sua natura - può essere suscettibile di diverse interpretazioni, mentre il gesto - nella sua semplicità - è sempre chiaro e quindi definitivo. Un no detto a voce, a seconda del tono, può anche significare 'sì, forse', o comunque rivelare una disponibilità alla trattativa; un no manifestato ruotando la testa a destra e sinistra, o ancora di più - come fanno nel sud d'Italia - un no espresso sollevando leggermente il mento e facendo un piccolo schiocco con la lingua, sembra assolutamente definitivo. Pragmaticamente sono marcatori dell'atteggiamento del parlante nei confronti di ciò che sta dicendo e manifestano le sue aspettative nei confronti di come il destinatario deve intendere le sue parole.

I gesti, insomma, funzionano come tutte le convenzioni sociali (se gli italiani trovano scorretti i rumori corporei in pubblico in altri $\mathrm{Pa}$ esi come la Thailandia sono riconosciuti come manifestazione di gradimento e di soddisfatta sazietà), rendono una conversazione più partecipata, possono essere ironici.

Nei rapporti interculturali non va dimenticato che mentre la mimica facciale è spesso interpretabile anche da culture diverse, i gesti hanno in molti casi significati diversi a seconda delle etnie e delle tradizioni culturali. La mimica facciale ha valore emotivo, è una manifestazione immediata, spontanea e involontaria delle emozioni, ha un significato oggettivo indipendente dal contesto situazionale (espressione di gioia o di dolore universalmente intelligibile) e una forte funzione comunicativa: manifestazione più o meno controllata e volontaria di emozioni, intenzioni, atteggiamenti e obiettivi dell'individuo, ogni espressione ha un significato variabile in funzione del contesto situazionale (espressione incerta, ironica, maliziosa). Le espressioni del volto veicolano grosso modo gli stessi stati emo- 
tivi in tutte le culture dato che si sono sviluppate a causa della loro utilità per la sopravvivenza; corrispondono alle sei emozioni di base (rabbia, paura, felicità, tristezza, sorpresa, disgusto) che vengono universalmente riconosciute in culture molto diverse (Ekman 1989).

Il sorriso, che in Europa occidentale e meridionale è segno di benevolenza e di assenso, in alcune culture orientali è segno di imbarazzo e a volte cela il dissenso. Gli studi sulla classificazione dei movimenti facciali hanno individuato diciannove configurazioni diverse di sorriso. È legato a una manifestazione volontaria o involontaria di emozioni, riveste importanti funzioni nell'interazione sociale in quanto regolatore dei rapporti sociali, promotore dell'affinità relazionale, strumento informativo.

Intensità, durata e direzione dello sguardo variano invece in relazione ai contesti e al grado di intimità (familiare o estraneo), alle emozioni sottostanti (gioia, imbarazzo, vergogna, disgusto), al valore sociale in un dato contesto culturale (la fissazione oculare può avere valore di sfida, sincerità, pericolo, minaccia). Ha funzione di feedback in quanto segnale d'appello per comunicare la propria disponibilità a iniziare un'interazione 0 , nella conversazione già avviata, di regolatore della turnazione.

Hall $(1959 ; 1966)$ definisce la distanza interpersonale «la dimensione nascosta» (hidden dimension), distinzione che consente di effettuare confronti fra culture diverse riguardo ai sistemi prossemici - sistemi di percezione, organizzazione e uso dello spazio e della distanza interpersonale come nella nota classificazione in: zona intima (da 0 a $50 \mathrm{~cm}$ ), zona personale (da $50 \mathrm{~cm}$ a $1 \mathrm{~m}$ ), zona sociale (da $1 \mathrm{~m}$ a 3-4 m), zona pubblica (oltre i $4 \mathrm{~m}$ ). Il rispetto o il venir meno del rispetto della distanza spaziale assume pertanto importanti significati a livello comunicativo. Oltre alla distanza anche l'orientazione, cioè l'angolo con cui le persone si situano l'una rispetto all'altra, e la postura sono segnali (in)volontari che variano soprattutto in relazione allo stato emotivo e lungo la dimensione rilassamentotensione, con specifici significati.

Il sistema vestemico è un sistema semantico dell'apparenza fisica, in relazione all'abbigliamento e agli ornamenti, concorre alla creazione dell'immagine di sé in funzione dei rapporti interpersonali, da quelli intimi a quelli pubblici: ogni cultura attribuisce un valore al modo di vestirsi, al trucco e agli oggetti indossati, tale da influenzare l'interazione. Nella comunicazione, parte dell'efficacia relazionale è affidata alla vestemica (cf. le relazioni di dominanza e di persuasione espresse dal valore delle divise, le collane e le frange degli hippies ecc.) che è immediata, preverbale.

Il sistema cronemico riguarda la percezione, l'organizzazione e l'uso del tempo per la scansione delle attività e dell'esperienza individuali. Dipende da specifici ritmi personali, fisiologici e psicologici e da valori e suddivisioni di una determinata cultura. 
Infine il sistema aptico è il sistema del contatto fisico, cioè l'insieme delle azioni di contatto che possono intervenire tra gli interlocutori di un atto comunicativo (mano sulla spalla, schiaffetto, carezza ecc.), si realizza con forme comunicative codificate (la stretta di mano, il bacio sulle guance come saluto ad amici e parenti), di natura più spontanea (un abbraccio, una pacca sulla spalla) o di necessità (toccarsi con il gomito per evitare contagi); varia profondamente da cultura a cultura ed ha un ruolo cruciale: ad es. la quantità di contatto fisico presente nei rapporti interpersonali fra le persone di cultura sud-europea verrebbe considerata come una violenta forma di invadenza dai popoli nord-europei, toccare la testa di un bambino è tabù in culture arabe ecc.

Gestire la diversità plurilingue e pluriculturale dei contesti interlinguistici e interculturali significa adottare una concezione del locutore realistica del locutore, del concetto di repertorio linguistico e chiamare in causa l'educazione plurilingue che dovrebbe sviluppare una competenza non limitata al solo aspetto strettamente linguistico della comunicazione. Purtroppo le relazioni tra politica linguistica educativa e didattica delle lingue non sono così semplici come il 'ruolo tecnico' della didattica potrebbe lasciare intendere (Beacco 2016): spetta alla didattica delle lingue discutere sulla pertinenza delle finalità assegnate dalla politica all'insegnamento delle lingue sia dal punto di vista educativo (qual è il ruolo dell'apprendimento delle lingue per lo sviluppo della persona?) sia dal punto di vista contestuale (quali finalità sono adeguate alle evoluzioni sociali e al fenomeno migratorio che investe globalmente le società?) eppure la politica linguistica viene gestita dai governi.

La didattica delle lingue elabora, con modalità proprie di ricerca, finalità che possono essere oggetto di decisioni politiche e servire da fondamento per il rinnovamento dell'insegnamento nel sistema educativo (riflessioni su educazione plurilinguistica ed educazione interculturale, nozione di contesto, competenze, generi di discorsi ecc.). Il terzo settore sta esplorando ambiti e nuove frontiere per far interagire attori diversi della società, ponendo l'attenzione su temi legati all'inclusione sociale, al plurilinguismo, alle metodologie di insegnamento e formazione degli individui. Dunque la didattica delle lingue si è spostata, almeno in Europa, da una posizione periferica della formazione verso una posizione centrale allargando i propri interessi dalla formazione degli individui in contesto scolastico a quella lungo tutto l'arco della vita e da un apprendimento indifferenziato ad uno individualizzato, rivolto alla formazione di competenze di vari livelli, per varie situazioni e soprattutto per differenti parlanti, tra i quali anche i pubblici 'svantaggiati' ai quali si è già accennato nel capitolo 1 (con differenti modelli europei di integrazione: assimilazionista francese, multiculturalista britannico o tedesco, interculturale frammentario italiano ecc.). Inoltre collegandosi ad alcuni stu- 
di sviluppatisi all'interno degli interessi della sociolinguistica e della sociologia delle comunicazioni, prende in esame altri aspetti del contatto delle lingue e della costruzione identitaria collettiva e individuale come quello del dominio teorico-metodologico dell'insicurezza linguistica' (Guenier 2002) del parlante, ambito di ricerca pluridisciplinare ed eterogeneo sviluppatosi soprattutto in area francofona, e quello della forza culturale della lingua (interesse sorto già dagli anni Quaranta del secolo scorso negli Stati Uniti e che richiama alla mente almeno i nomi di Lado, Labov, Whorf, Sapir) arricchitosi più recentemente con le numerose riflessioni sull'analisi del discorso di tipo conversazionale-interazionale (Simonin 1996) e su uno spazio di applicazione plurilingue più ampio (Calvet 1998).

In questa cornice dalla duplice dimensione psicologica (sentimenti ed emozioni) e linguistica (comportamenti e realizzazioni di forme di comunicazione), corrispondente ad un doppio piano oggettivo e soggettivo, qualitativo e quantitativo, descrittivo e interpretativo, assume rilevanza anche il concetto di comunità linguistica, in quanto unità di parlanti che interagiscono secondo un rituale di norme, codici, segni e categorizzazioni comunemente riconosciuti e codificati (Bretegnier 2002). L'insicurezza linguistica nasce da un sentimento di esclusione o una richiesta di riconoscimento da parte di parlanti 'svantaggiati' quali gli immigrati (almeno alcune categorie tra di essi) e comprende sia gli aspetti linguistici che i sistemi di convenzioni socialmente costruite, non condivise o non conosciute, e quelli non verbali. L'insieme di questi aspetti che connotano le situazioni di lingue e culture in contatto, e in particolare i rapporti interlinguistici in contesti plurilingui, stanno cambiando anche le dinamiche e le strategie monolinguistiche della comunicazione e della formazione linguistica poiché obbligano a prendere in considerazione anche la sfera più personale, identitaria, in rapporto alla/e lingua/e di contatto e conseguentemente l'aspetto interrelazionale legato al senso di inadeguatezza, disuguaglianza, alla disparità di ruoli, funzioni e atteggiamenti dei parlanti (immigrati) (Benucci 2020, 185-7).

Qualsiasi rapporto tra persone di Paesi diversi presenta sempre una dicotomia da cui non si può prescindere, un 'noi' e un 'voi' che si accentua quando si tratta di aspetti culturali:

Vicino al bar dell'aeroporto c'è un forte odore di caffè. La cosa più sgradevole in Italia è proprio il caffè! Così amaro, duro, ancora adesso, dopo tanti anni, il suo aroma mi fa fuggire! Noi non beviamo questo tipo di caffè ma un altro, più soffice, più lungo, che si adatta alla sete del deserto. (da R.R. Zargar «Finalmente sono qui», Cittadini, 18 novembre 2011)

Chiunque si trovi a gestire un rapporto con l'‘altro', anche se è un esperto di mentorship, non può conoscere tutte le lingue e le cultu- 
re della migrazione ma ha almeno il dovere di essere informato sugli aspetti della comunicazione e dell'interculturalità perché questo serve per poter agire nel miglior modo possibile: apprenderà ad osservare l'altro tenendo conto di alcuni aspetti che generalmente non sono il focus di una formazione permanente, come i linguaggi verbali, non verbali, i valori e a sapersi orientare all'interno di determinate coordinate in cui possono crearsi malintesi e criticità. La conoscenza del codice lingua non è sufficiente per le azioni di mediazione e interazione, conoscere l'arabo non basta per orientarsi in tutti i Paesi in cui si parla l'arabo e soprattutto tra tutte le sue varianti dialettali.

Gli interpreti formati per rispondere alle esigenze dei migranti, oltre a conoscere bene le loro L1 e le tecniche di interpretariato, devono essere capaci di intervenire in ambito sanitario, giuridico ecc., in concrete situazioni di interazione mediata. I mediatori sono essi stessi nella maggior parte dei casi persone che hanno un passato di migrazione, sono bilingui, si considerano vicini alle esperienze degli ospiti e ritengono che ciò basti per spiegare differenze di uso, di prassi e di funzionamento di servizi pubblici ecc.

Interpretare un discorso è comunque una mediazione, un intervento di un soggetto sul pensiero di un altro soggetto ed è tarato su un modello dialogico, di turno di parola che l'interprete traduce consecutivamente, ma che non risponde alle regole della realtà della comunicazione. È molto difficile ristabilire la reciprocità, possibile soltanto se si ricorre a spiegazioni preliminari, a spazi per far manifestare l'orientamento da parte dei parlanti e con chiarimenti dei propri punti di vista. La conversazione va avanti se non meno di due persone mostrano di farsi capire, palesando la comprensione e ricostruendo attraverso l'interprete/mediatore la manifestazione della comprensione: si rende quindi necessario 'comunicare sulla comunicazione' da parte dell'interprete/mediatore e ciò non si realizza solo con la traduzione dei singoli turni di parola. ${ }^{1}$

Di recente si è costituito un gruppo di studio dell'interpretazione di pubblico servizio in collaborazione con associazioni che si occupano della Lingua dei Segni Italiana (LIS) dato che malgrado la LIS si rivolga primariamente a persone con disturbi di udito i suoi principi sono utili per affrontare anche altri tipi di difficoltà comunicativa, per superare equivoci linguistici, fraintendimenti, difficoltà nella comprensione delle dinamiche relazionali poiché l'incapacità di instaurare con gli altri una relazione significativa non è causata dalla sordità in se per sé, bensì dalle barriere che impediscono la comunicazione. I lavori fino ad ora svolti portano a confermare una epistemologia

1 Gavioli ha indagato a fondo questi aspetti in varie occasioni, si vedano per es. Gavioli, Baraldi 2011; Baraldi, Gavioli 2010; 2008; Gavioli 2009; Gavioli, Zorzi 2008; Amato, Gavioli 2008; Banfi et al. 2006. 
della comunicazione ormai condivisa anche con i glottodidatti. Per codificare, scambiare e decodificare messaggi è infatti necessario:

1. possedere adeguate competenze verbali e non verbali e saperle integrare;

2. saper operare sui testi che in concreto realizzano i messaggi, cioè saperli comprendere (ascoltare e leggere); saperli produrre in prima persona (monologare e scrivere) o in maniera interattiva (dialogare e scrivere sotto dettatura); saperli manipolare (riassumere, parafrasare, tradurre), cioè avere padronanza dei processi che soggiacciono alle abilità linguistiche;

3. saper padroneggiare la forza pragmatica dei messaggi (saper fare con la lingua) cioè possedere competenze pragmatica e sociolinguistica per gestire atti di parola ma anche varietà di registri e tararli sulle situazioni comunicative.

La comunicazione può essere poi collocata su un continuum che va da 'approssimazione' a 'esattezza': ad un'estremità si trova la comunicazione quotidiana, all'altra quella microlinguistica; per la prima sono sufficienti competenze in lingua comune, per la seconda occorrono conoscenze di specifici settori del sapere, tecniche e specializzate, che vanno oltre i lessici delle microlingue. L'ormai nota suddivisione delle competenze enunciate nel QCER (2002), seppur di venti anni fa, ben si adatta a fare da corollario a questa concezione della comunicazione perché devono essere oggetto di riflessione non soltanto da parte del docente di lingua o del redattore di materiali per la didattica ma anche dell'interprete/mediatore.

Secondo il QCER le competenze generali vanno declinate sulla base delle singole culture riguardo al:

- sapere - conoscenza del mondo, consapevolezza interculturale;

- saper fare - capacità di agire nella comunità di riferimento del migrante;

- saper essere - atteggiamenti, personalità, motivazioni, stile cognitivo ecc. che predispongono il terreno di mediazione;

- saper apprendere - avere stili e strategie di apprendimento che permettono di ampliare le competenze in base alle occorrenze e di fare tesoro delle esperienze pregresse.

A queste competenze si aggiungono quelle linguistico-comunicative:

- linguistiche - lessicale, grammaticale, semantica, fonologica, ortografica, ortoepica;

- sociolinguistiche - formule di saluto, regole di cortesia, differenze di registro ecc.;

- pragmatiche - discorsiva, funzionale ecc.

La lingua va però intesa come un insieme dinamico che varia a seconda di quattro caratteristiche fondamentali, secondo la terminologia più correntemente usata in sociolinguistica: 
- varietà diatopica, ovvero il mutamento in base alla posizione geografica (più accentuato in alcuni Paesi come l'Italia) che obbliga a fare i conti (e a operare scelte non dettate da aprioristiche idee di prestigio) con i dialetti e altre varietà locali a detrimento dello standard, spesso idealizzato o limitato ai soli parlanti molto competenti e a certi ambiti istituzionali;

- varietà diastratica, determinata dallo status sociale del parlante, dal suo livello di istruzione e sociale (poco evidente in italiano ma non in altre lingue comprese alcune tra quelle parlate da molti immigrati);

- varietà diafasica, che comporta scelte - almeno da parte di parlanti competenti in grado di utilizzare tutto lo spazio linguistico a loro disposizione - in base alla situazione comunicativa, al contesto, al rapporto tra gli interlocutori;

- varietà diamesica, dipendente dal canale di comunicazione utilizzato, principalmente scritto o orale ma negli ultimi decenni sempre di più 'trasmesso'.

Particolarmente studiato è l'ambito della comunicazione sanitaria, di non secondaria importanza per la problematica del contatto interlinguistico e interculturale nelle istituzioni delle società contemporanee investite dal fenomeno delle migrazioni. Quasi tutte le ricerche sulla relazione e sulla comunicazione medico-paziente immigrato hanno un carattere transdisciplinare (per es. gli studi di Rechel et al. 2011; Whittal, Lipke 2015; Orletti, Fatigante 2013), lo studio di Benucci e Grosso (2021) ha invece analizzato la percezione soggettiva e sociale dell'efficacia della comunicazione reciproca in ambito sanitario e la natura e/o l'assenza della semplificazione linguistica mettendo in relazione due dimensioni, psicologica e culturale in termini antropologici, così da affrontare la diversità di ogni individuo all'interno di una cultura e la diversità delle singole culture, e comprendere come le risposte che le differenti culture elaborano per risolvere i bisogni - e che costituiscono il sostrato comune dell'identità culturale di un determinato soggetto in rapporto al gruppo dei pari, alla collettività, all'etnia ecc. - possano sfociare nel disagio o nel pregiudizio a cui si dà forma con la parola, la comunicazione, l'interazione o la loro negazione.

L'altro ambito comunicativo oggetto di attenzione negli ultimi anni è quello dell'inserimento professionale di soggetti migranti per il quale molto ben si addicono i principi del Content Language Integrated Learning (CLIL), dato che l'impostazione di corsi CLIL per adul-

2 La definizione di trasmesso si deve a Sabatini (1990) che alcuni anni fa ne spiegava le caratteristiche, comprendendo con questa etichetta ogni forma di comunicazione veicolata da mezzi tecnici come il telefono, il fax, la radio, la televisione e oggi soprattutto Internet e la telefonia mobile. 
ti stranieri può essere meno rigidamente concepita e realizzata di quelli scolastici, con una più stretta concatenazione tra teoria - apprendimento linguistico e pratica - apprendimento di una professione. In questo caso sono di particolare importanza inventari per generi discorsivi, l'attenzione alla dimensione testuale costituisce la riflessione preliminare per veicolare i contenuti disciplinari e permette di elaborare sillabi di lingua settoriale, ipotizzare contenuti lessicali attendibili, selezionare strutture morfosintattiche necessarie per un determinato livello di competenza tralasciandone altre, individuare aree funzionali legate ai generi testuali caratterizzanti la didattica per scopi settoriali (disciplinare in ambito scolastico). Un approccio CLIL per corsi settoriali ad adulti è in grado di produrre motivazione dato che il contenuto della materia diviene il focus dell'attenzione, offre anche la possibilità di aumentare la quantità e qualità di esposizione alla L2: l'insegnamento integrato di lingua e contenuti privilegia un'educazione di tipo interattivo e assicura un certo livello qualitativo perché richiede una profondità di rielaborazione che non si realizzerebbe in un insegnamento di tipo tradizionale. È preferibile impostarlo facendo svolgere attività autentiche, meglio se è associato all'attività di training professionale, perché permette di usare la lingua in un contesto reale, di usare una lingua autentica al servizio di azioni specifiche in determinate attività lavorative e con argomenti ben definiti: si sposta così l'attenzione dalla forma linguistica ai contenuti da essa veicolati, sono infatti fondamentali l'input e il contenuto, non la correttezza a livello formale. Una tale formazione permette di raggiungere buoni livelli di interazione, pur in presenza di errori e imprecisioni linguistiche, perché incita l'apprendente a ricorrere ad altri codici (per es. quelli cinesico e gestuale) come avviene normalmente nelle interazioni di lavoro, in particolar modo quelle che comportano lo svolgimento di concrete azioni come nelle attività lavorative più frequentemente scelte dai migranti.

L'elaborazione di sillabi e materiali per scopi settoriali-professionali comporta una assunzione del riconoscimento, e del pieno diritto di essere sufficiente, della parzialità di competenze e conoscenze (per es. saper comprendere una lingua vicina, conoscere il lessico specialistico della propria professione, possedere un livello A1 in una L2 per la produzione orale ma un B2 per la lettura): l'adulto ricorre in base alla situazione comunicativa e ai saperi pregressi (expectancy grammar) alle competenze linguistiche parziali che possiede, di grande aiuto almeno nei primi momenti dell'inserimento sociale e lavorativo. Il concetto di parzialità, che investe anche le competenze dei nativi perché rispecchia la realtà dei fatti contro una utopistica idea della competenza 'completa', potenzia anche l'autopromozione se si accetta che il migrante possieda un repertorio in L2 spesso qualitativamente poco elevato (varietà regionali, locali, dialetti) e 
quantitativamente limitato al dominio lavorativo ma ben contestualizzato (indici extralinguistici) e adeguato alle esperienze di vita. ${ }^{3}$

Da un punto di vista più propriamente didattico ciò significa adottare un approccio plurilingue e pluriculturale e procedure didattiche e strumenti che tengano conto di una scarsa disponibilità di tempo (allenamento) per lo studio e della discontinuità nella frequenza delle lezioni. Di conseguenza richiede una attenta scelta del livello di difficoltà: per esempio in alcuni casi si possono proporre testi con contenuti più complessi di quelli che l'ordine di acquisizione naturale implicherebbe, in virtù della loro importanza ai fini comunicativi e funzionali, a patto di non richiedere di processarli nel dettaglio. Infatti a livello di comprensione si possono selezionare strutture più complesse sulle quali non si richiede una produzione, per la produzione accettare esecuzioni con errori ma pragmaticamente effica$\mathrm{ci}$, presentare e richiedere di produrre come fossero formule (senza processarle) certe espressioni difficili per il livello di competenza comunicativa posseduto ma necessarie per l'interazione in ambito lavorativo. I dati a disposizione mostrano che se il corso in linguaggio settoriale è seguito immediatamente dal tirocinio la ritenzione di quanto appreso è molto alta.

I materiali didattici orientati in questo senso sono ormai molti, si cita qui quello di Benucci e Maiorano (2015) relativo all'apprendimento linguistico e professionale in italiano L2 i cui destinatari sono gli adulti arrivati in Italia, inseriti o di prossimo inserimento nel mercato del lavoro; adolescenti e giovani adulti arrivati in Italia che hanno ottemperato all'obbligo scolastico, inseriti o di futuro inserimento nel mercato del lavoro; studenti stranieri iscritti agli ultimi anni di scuole professionali; stranieri in carcere, in vista di un reinserimento socio-lavorativo alla fine della detenzione. Ogni Quaderno riguarda un singolo profilo professionale ed è composto di tre Unità di Apprendimento, per corsi di circa 30 ore possibilmente erogati congiuntamente al tirocinio aziendale. Il materiale didattico ricorre ampiamente alla comunicazione non verbale con immagini funzionali su cui sono impostate molte attività, contiene attività comunicative e pragmatiche, sollecitazioni di riflessione interculturale, e pochi esercizi strutturali.

Associato a questi materiali didattici è il Portfolio linguistico-professionale elaborato nel corso di due progetti (DEPORT e RiUscire $)^{4}$

3 Si vedano i sillabi prodotti per lavoratori immigrati con livello target B2 parziale (Benucci 2014): operatori sanitari e addetti alla cura della persona, operai del settore agricolo, addetti alle pulizie e operatori ecologici, operatori del settore edile, personale non qualificato nell'industria, profilo di aiuto cuoco e addetti ai servizi di ristorazione, operatori dell'artigiano e del commercio, collaboratori domestici e assistenti familiari.

4 I progetti DEPORT (Oltre i confini del carcere: portfolio linguistico-professionale per detenuti, PAR FAS 2007-2013, Linea di azione 1.1.a.3, 2012-2015) e RiUscire (Rete 
suddiviso in: «Copertina», la presenza di una copertina ha lo scopo di personalizzare al massimo il Portfolio sottolineando che esso è di proprietà della persona che lo ottiene ed implica quindi un suo ruolo attivo e quindi una motivazione intrinseca, intende sviluppare un senso di autostima, promuove la percezione di auto-efficacia nel soggetto che lo consegue; la «Biografia linguistica e culturale» che raccoglie tutte le informazioni che riguardano la lingua di origine e le esperienze linguistiche della persona e l'Autovalutazione delle competenze linguistiche (esclusa la lingua d'origine e l'italiano); la «Valutazione delle competenze professionali» che descrive le competenze acquisite durante il tirocinio (profilo professionale, aspetti culturali e di cittadinanza, situazioni / azioni / aspetti culturali) e un Dossier che include la valutazione sulle competenze linguistiche relative al settore di svolgimento del tirocinio; la «Valutazione delle competenze professionali», redatta dall'azienda che ospita il tirocinante, con la descrizione delle situazioni e delle azioni in cui il lavoratore può implementare le competenze linguistico-professionali apprese durante il corso di formazione. Seguono due Appendici, una con i descrittori del QCER, l'altra con l'elenco delle competenze linguistico-professionali suddivise per comprensione, strutture comunicative, parlato, produzione scritta.

Si fornisce un esempio nelle pagine seguenti.

Universitaria SocioCulturale per l'Istruzione e il Recupero in Carcere, Erasmus+ - Ka2 SETTORE ADULTI, 2014-2017) si rivolgevano principalmente a un pubblico di immigrati detenuti ma hanno prodotto riflessioni e materiali anche per altre tipologie di immigrato (cf. Benucci, Grosso 2015; 2017; Bormioli 2017; Benucci 2017; Benucci, Birello 2017; Sciuti Russi, Carmignani 2015). 


\section{Profilo}

OPERAIO AGRICOLO/MANUTENTORE DEL VERDE

Nome

Cognome

Luogo e data di nascita

Nazionalità

a. Biografia linguistica e culturale: raccoglie tutte le informazioni che riguardano la lingua d'origine e le esperienze linguistiche della persona (scuola, professione, ecc...)

1. Lingua/e d'origine

\begin{tabular}{|c|c|c|c|c|c|}
\hline $\begin{array}{l}\text { Lingua/e } \\
\text { d'origine }\end{array}$ & Alfabetizzato & $\begin{array}{l}\text { Non } \\
\text { alfabetizzato }\end{array}$ & Anni di studio & $\begin{array}{l}\text { Anno e luogo di } \\
\text { conseguimento }\end{array}$ & $\begin{array}{l}\text { Nome } \\
\text { dell'Istituto }\end{array}$ \\
\hline
\end{tabular}

2. Autovalutazione delle competenze linguistiche (esclusa la lingua d'origine e ('italiano)

\begin{tabular}{|c|c|c|c|c|c|c|}
\hline $\begin{array}{l}\text { Lingue } \\
\text { conosciute }\end{array}$ & $\begin{array}{l}\text { Livelli di } \\
\text { competenza }\end{array}$ & Ascolto & Lettura & Interazione & $\begin{array}{l}\text { Produzione } \\
\text { scritta }\end{array}$ & $\begin{array}{l}\text { Produzione } \\
\text { orale }\end{array}$ \\
\hline & $\mathrm{A} 1$ & & & & & \\
\hline & A2 & & & & & \\
\hline & B1 & & & & & \\
\hline & B2 & & & & & \\
\hline & $\mathrm{C} 1$ & & & & & \\
\hline & C2 & & & & & \\
\hline
\end{tabular}




\section{b. Valutazione delle competenze professionali}

\begin{tabular}{l} 
Profilo professionale \\
\hline Operaio agricolo/manutentore del verde \\
Descrizione sintetica della figura: \\
L'operatore agricolo interviene, a livello esecutivo, \\
nel processo lavorativo con autonomia e responsabilità \\
limitate a ciò che prevedono le procedure e le \\
metodiche della sua operatività. La qualificazione \\
nell'applicazione e utilizzo di metodologie di base, \\
di strumenti e di informazioni gli consentono di \\
collaborare nella gestione dell'azienda e di svolgere, \\
a seconda dell'indirizzo, attività relative \\
all'allevamento di animali, alle coltivazioni arboree, \\
alle coltivazioni erbacee, all'orto/floricoltura, \\
alla silvicoltura e salvaguardia dell'ambiente, \\
con competenze nello svolgimento delle operazioni \\
fondamentali attinenti alla produzione zootecnica \\
e vegetale, nonché nell'esecuzione di operazioni \\
nella trasformazione di prodotti primari dell'azienda \\
e nella tutela e protezione dell'ambiente naturale.
\end{tabular}

Situazioni/azioni/aspetti culturali

\section{Aspetti culturali e di cittadinanza}

a. Conoscenza e competenza nelle principali colture e tecniche di coltivazione adottate in Italia:

- agricoltura, anche biologica e biodinamica;

- modalità di coltivazione;

- tipologia di piante.

b. Conoscenza dei territori, paesaggi e abitudini colturali in Italia:

- spazi verdi pubblici e privati;

- natura, ecologia e cicli stagionali;

- paesaggi regionali e geografia fisica dell'Italia.

c. Conoscenza e competenza nell'uso dei criteri

di formalità e informalità in contesti interazionali differenziati:

- criteri di formalità e informalità in rapporto all'età, alla situazione, al ruolo sociale e al sesso: formule di saluto, prossemica, alternanza turni di parola, tono della voce;

- gerarchie di status e rispetto dei ruoli;

- tipologia contrattuale;

- sistema della sicurezza e sanità.

1. Accoglienza e svolgimento attività in cooperativa/ azienda: interazioni con impiegati dell'ufficio; interazioni con i colleghi.

2. Formazione antinfortunistica e rispetto delle norme vigenti in azienda.

3. Lavoro in squadra, individuale e con tutor aziendale nello svolgimento delle diverse mansioni (capacità di relazionarsi con i colleghi), ovvero:

- raccolta frutta/ortaggi;

- potatura e raccolta;

- realizzazione piccoli giardini, ordinaria manutenzione di un giardino, uso corretto di attrezzature e materiali specifici del giardinaggio (ad es., un impianto di irrigazione, un decespugliatore ecc.);

- lavorazione alla fertilizzazione dei terreni, alle semine, ai trattamenti fitosanitari.

4. Riunioni e incontri con la Direzione per il monitoraggio del tirocinio/lavoro (capacità di relazionarsi con i superiori).

5. Aspetti culturali legati alla professione:

a. conoscenza e competenza nelle principali colture e tecniche di coltivazione adottate in Italia; b. conoscenza dei territori, paesaggi e abitudini colturali in Italia;

c. conoscenza e competenza nell'uso dei criteri di formalità e informalità in contesti interazionali differenziati. 
3) Valutazione delle competenze professionali: descrive le situazioni e le azioni in cui il lavoratore può implementare le competenze linguistico-professionali apprese durante il corso di formazione attraverso la Scheda di descrizione situazioni/azioni.

\begin{tabular}{|c|c|c|}
\hline & Situazioni/azioni/aspetti culturali & \\
\hline \multirow{5}{*}{$\begin{array}{l}\text { Profilo: } \\
\text { Operaio agricolo/ } \\
\text { manutentore } \\
\text { del verde }\end{array}$} & $\begin{array}{l}\text { 1. Accoglienza e svolgimento di attività in cooperativa/azienda: } \\
\text { interazioni con gli impiegati dell'ufficio; interazioni con i colleghi. }\end{array}$ & $\square$ \\
\hline & $\begin{array}{l}\text { 2. Formazione antinfortunistica e rispetto delle norme vigenti } \\
\text { in azienda. }\end{array}$ & $\square$ \\
\hline & $\begin{array}{l}\text { 3. Lavoro in squadra, individuale e con tutor aziendale nello svolgimento } \\
\text { delle diverse mansioni (capacità di relazionarsi con i colleghi), ovvero: } \\
\text { - raccolta frutta/ortaggi; } \\
\text { - potatura e raccolta; } \\
\text { - realizzazione piccoli giardini, ordinaria manutenzione di un giardino, } \\
\text { uso corretto di attrezzature e materiali specifici del giardinaggio } \\
\text { (ad es., un impianto di irrigazione, un decespugliatore ecc.); } \\
\text { - lavorazione alla fertilizzazione dei terreni, alle semine, ai trattamenti } \\
\text { fitosanitari. }\end{array}$ & $\square$ \\
\hline & $\begin{array}{l}\text { 4. Riunioni e incontri con la Direzione per il monitoraggio del tirocinio/ } \\
\text { lavoro (capacità di relazionarsi con i superiori). }\end{array}$ & $\square$ \\
\hline & $\begin{array}{l}\text { 5. Aspetti culturali legati alla professione: } \\
\text { a. conoscenza e competenza nelle principali colture e tecniche } \\
\text { di coltivazione adottate in Italia; } \\
\text { b. conoscenza dei territori, paesaggi e abitudini colturali in Italia; } \\
\text { c. conoscenza e competenza nell'uso dei criteri di formalità } \\
\text { e informalità in contesti interazionali differenziati. }\end{array}$ & $\square$ \\
\hline
\end{tabular}




\section{Appendice 1}

Sezione a.2 - Biografia linguistica (autovalutazione competenze) - Descrittori lingue conosciute oltre alla lingua/e d'origine (escluso l'italiano) (fonte: QCER 2002)

\begin{tabular}{cll}
\hline & A1 & A2 \\
\hline ASCOLTO & Riconosco parole & Comprendo \\
(A) & familiaried espressioni espressioni che \\
& molto semplicisu me & sono usate spesso \\
& stesso se la persona & e che mi riguardano \\
& parla lentamente. & (informazioni su di \\
& me, la mia famiglia, \\
& gli acquisti, il luogo di \\
& lavoro).
\end{tabular}

\section{B1}

Riesco a capire gli elementi principali in un discorso chiaro su argomenti familiari, che incontro spesso al lavoro, nel tempo libero ecc. lavoro).

\begin{tabular}{|c|c|c|c|c|c|}
\hline $\begin{array}{l}\text { LETTURA } \\
(\mathrm{L})\end{array}$ & $\begin{array}{l}\text { Capisco testi brevi } \\
\text { su cose che mi sono } \\
\text { familiari. Capisco frasi } \\
\text { molto semplici, } \\
\text { per esempio quelle } \\
\text { diannunci, cartelloni e } \\
\text { cataloghi. }\end{array}$ & $\begin{array}{l}\text { Riesco a leggere testi } \\
\text { brevi e semplici che } \\
\text { posso trovare nella } \\
\text { vita di tutti i giorni. } \\
\text { Riesco anche a } \\
\text { trovare informazioni } \\
\text { particolari in questi } \\
\text { testi. }\end{array}$ & $\begin{array}{l}\text { Riesco a capire testi } \\
\text { scritti che riguardano } \\
\text { gli aspetti della vita } \\
\text { quotidiana o del } \\
\text { lavoro. }\end{array}$ & $\begin{array}{l}\text { Riesco a leggere } \\
\text { articoli su argomenti } \\
\text { d'attualità in cui } \\
\text { l'autore spiega } \\
\text { un punto di vista } \\
\text { specifico. }\end{array}$ & $\begin{array}{l}\text { Riesco a capire testi } \\
\text { di autori importanti } \\
\text { e testi con molte } \\
\text { informazioni lunghi } \\
\text { e particolari. Riesco } \\
\text { a capire tutti i dettagli. }\end{array}$ \\
\hline $\begin{array}{l}\text { INTERAZIONE } \\
\text { (INT.) }\end{array}$ & $\begin{array}{l}\text { Riesco a parlare in } \\
\text { modo semplice con } \\
\text { un'altra persona, } \\
\text { se la persona parla } \\
\text { lentamente, in modo } \\
\text { chiaro e semplice, e se } \\
\text { mi dice di nuovo più } \\
\text { lentamente certe cose } \\
\text { che non capisco bene. }\end{array}$ & $\begin{array}{l}\text { Riesco a parlare con } \\
\text { un'altra persona, } \\
\text { so fare domande e } \\
\text { rispondere in modo } \\
\text { semplice e diretto su } \\
\text { argomenti e attività } \\
\text { che conosco. }\end{array}$ & $\begin{array}{l}\text { Riesco a partecipare, } \\
\text { senza essermi } \\
\text { preparato, a } \\
\text { conversazioni su } \\
\text { argomenti familiari, } \\
\text { che mi interessano o } \\
\text { che riguardano la vita } \\
\text { di tutti i giorni. }\end{array}$ & $\begin{array}{l}\text { Riesco a partecipare } \\
\text { a una conversazione, } \\
\text { spiegando le mie } \\
\text { opinioni, facendo } \\
\text { anche esempi giusti. }\end{array}$ & $\begin{array}{l}\text { Riesco a parlare in } \\
\text { modo naturale } \\
\text { e chiaro senza dover } \\
\text { cercare troppo le } \\
\text { parole. Riesco ad usare } \\
\text { la lingua nel modo } \\
\text { adatto alle situazioni } \\
\text { e nel modo giusto } \\
\text { per avere l'effetto che } \\
\text { voglio quando parlo } \\
\text { con altre persone } \\
\text { nel tempo libero } \\
\text { o al lavoro. }\end{array}$ \\
\hline $\begin{array}{l}\text { PRODUZIONE } \\
\text { ORALE } \\
\text { (PO) }\end{array}$ & $\begin{array}{l}\text { Riesco a usare } \\
\text { espressioni e frasi } \\
\text { semplici per descrivere } \\
\text { me stesso, la gente } \\
\text { che conosco e il luogo } \\
\text { dove abito. }\end{array}$ & $\begin{array}{l}\text { Riesco a usare una } \\
\text { serie di espressioni } \\
\text { e frasi per descrivere } \\
\text { con parole semplici la } \\
\text { mia famiglia ed altre } \\
\text { persone, la mia vita e il } \\
\text { mio lavoro. }\end{array}$ & $\begin{array}{l}\text { Riesco a spiegare } \\
\text { brevemente quello } \\
\text { che penso. Riesco a } \\
\text { raccontare una storia } \\
\text { e la trama di un libro } \\
\text { o di un film. Riesco } \\
\text { a descrivere una } \\
\text { procedura legata al } \\
\text { mio lavoro. }\end{array}$ & $\begin{array}{l}\text { Riesco a esprimermi } \\
\text { in modo chiaro e } \\
\text { con molti particolari } \\
\text { su molti argomenti } \\
\text { che mi interessano. } \\
\text { Riesco a esprimere } \\
\text { un'opinione su un } \\
\text { argomento, spiegando } \\
\text { le diverse opzioni. }\end{array}$ & $\begin{array}{l}\text { Riesco a parlare } \\
\text { in modo naturale. } \\
\text { Riesco a presentare } \\
\text { descrizioni chiare } \\
\text { e con molte } \\
\text { informazioni su } \\
\text { argomenti particolari. }\end{array}$ \\
\hline $\begin{array}{l}\text { PRODUZIONE } \\
\text { SCRITTA } \\
\text { (PS) }\end{array}$ & $\begin{array}{l}\text { Riesco a compilare } \\
\text { moduli con dati } \\
\text { personali scrivendo } \\
\text { per esempio il mio } \\
\text { nome, la nazionalità } \\
\text { el'indirizzo. }\end{array}$ & $\begin{array}{l}\text { Riesco a prendere } \\
\text { semplici appunti } \\
\text { ea scrivere brevi } \\
\text { messaggi su argomenti } \\
\text { che conosco. Riesco } \\
\text { a scrivere una lettera } \\
\text { personale molto } \\
\text { semplice, per esempio } \\
\text { per ringraziare. }\end{array}$ & $\begin{array}{l}\text { Riesco a scrivere } \\
\text { in modo chiaro testi } \\
\text { semplici e con } \\
\text { un senso su argomenti } \\
\text { che conosco o che } \\
\text { mi interessano. Riesco } \\
\text { a scrivere lettere } \\
\text { personali dove scrivo } \\
\text { le mie esperienze e } \\
\text { quello che penso. }\end{array}$ & $\begin{array}{l}\text { Riesco a scrivere } \\
\text { testi chiari e con } \\
\text { molte informazioni } \\
\text { su argomenti molto } \\
\text { diversi che mi } \\
\text { interessano. } \\
\text { Riesco a scrivere saggi } \\
\text { e relazioni. }\end{array}$ & $\begin{array}{l}\text { Riesco a scrivere testi } \\
\text { chiari, spiegando } \\
\text { molto bene il mio } \\
\text { punto di vista. Riesco } \\
\text { ascrivere lettere, } \\
\text { saggi e relazioni } \\
\text { raccontando } \\
\text { argomenti particolari. }\end{array}$ \\
\hline
\end{tabular}

\section{C1}

Riesco a capire un discorso lungo anche $\begin{array}{ll}\text { lunghi. Riesco a } & \text { se non è organizzato } \\ \text { seguire ragionamenti in modo chiaro e i }\end{array}$ anche difficilise collegamentinonsono conoscoabbastanza dettidirettamente, ma l'argomento. Riesco a rimangono sottointesi. capire la maggior parte Riesco a capire dei telegiornaliedei abbastanzafacilmente giornali radio. Riesco a letrasmissioni capire le trasmissioni televisive i film. TV che riguardano fatti d'attualità, argomenti legatial mio lavoro ela maggior parte dei film. informazioni lunghi e particolari. Riesco a capire tutti i dettagli.

Riesco a partecipare Riesco a parlare in e chiaro senza dover cercare troppo le parole. Riesco ad usare e nel modo giusto per avere l'effetto che oglio quando parlo o al lavoro. descrizioni chiare con molte informazionisu argomenti particolari.

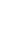


Benucci

2 - Comunicazione efficace in contesti interlinguistici e interculturali

\begin{tabular}{|c|c|c|c|c|c|}
\hline \multirow[t]{2}{*}{$\begin{array}{l}\frac{0}{c} \\
\frac{0}{n} \\
\frac{c}{0} \\
\frac{0}{0} \\
\frac{0}{\Xi}\end{array}$} & Ascolto & $\begin{array}{l}\text { Riconosco espressioni } \\
\text { e frasi molto semplici } \\
\text { riguardanti il mio lavoro } \\
\text { e le attrezzature che } \\
\text { utilizzo, a condizione che } \\
\text { l'esposizione sia lenta e ben } \\
\text { scandita. }\end{array}$ & $\begin{array}{l}\text { Riesco a comprendere le } \\
\text { principali parole e frasi } \\
\text { riguardanti il mio lavoro e } \\
\text { le attrezzature che utilizzo. } \\
\text { Riesco a capire l'essenziale } \\
\text { in brevi e semplici messaggi } \\
\text { televisivi e radiofonici } \\
\text { (inserzioni pubblicitarie, } \\
\text { bollettini) riguardanti il mio } \\
\text { settore lavorativo. }\end{array}$ & $\begin{array}{l}\text { Riesco a comprendere i } \\
\text { punti principali di discorsi } \\
\text { in lingua standard e } \\
\text { non standard (varietà } \\
\text { lessicali colloquiali e } \\
\text { regionali) con cui mi } \\
\text { trovo quotidianamente } \\
\text { a contatto riguardanti il } \\
\text { mio settore lavorativo e } \\
\text { le denominazioni delle } \\
\text { attrezzature in esso } \\
\text { comunemente utilizzate. } \\
\text { Riesco a capire gli elementi } \\
\text { principali di messaggi } \\
\text { televisivi e radiofonici } \\
\text { (inserzioni pubblicitarie, } \\
\text { annunci, bollettini) } \\
\text { riguardanti il mio settore } \\
\text { lavorativo, a condizione che } \\
\text { l'esposizione sia non troppo } \\
\text { veloce e ben scandita. }\end{array}$ & $\begin{array}{l}\text { Riesco a comprendere } \\
\text { esposizioni abbastanza } \\
\text { lunghe e a capire } \\
\text { argomentazioni anche } \\
\text { complesse in discorsi } \\
\text { riguardanti il mio settore } \\
\text { lavorativo, le denominazioni } \\
\text { delle attrezzature, i } \\
\text { macchinari e le novità } \\
\text { più recenti. Riesco a } \\
\text { capirevarietà lessicali } \\
\text { colloquiali e regionali e } \\
\text { alcuni geosinonimi più } \\
\text { frequenti relativi al mio } \\
\text { settore lavorativo. Riesco a } \\
\text { comprendere buona parte } \\
\text { dei messaggi televisivi } \\
\text { e radiofonici (inserzioni } \\
\text { pubblicitarie, annunci, } \\
\text { bollettini) riguardanti il mio } \\
\text { settore lavorativo. } \\
\text { Comprendo abbastanza } \\
\text { bene la maggior parte delle } \\
\text { consegne, delle istruzioni e } \\
\text { dei consigli espressi dai miei } \\
\text { superiori, senza necessità } \\
\text { che vengano ripetuti. }\end{array}$ \\
\hline & Lettura & $\begin{array}{l}\text { Riesco a comprendere le } \\
\text { principali parole riguardanti } \\
\text { il mio lavoro quotidiano; } \\
\text { riesco a capire l'essenziale } \\
\text { nel contenuto di prospetti, } \\
\text { schemi e orari molto brevi. }\end{array}$ & $\begin{array}{l}\text { Riesco a leggere brevi testi } \\
\text { riguardanti aspetti tipici } \\
\text { del mio lavoro quotidiano. } \\
\text { Riesco a comprendere } \\
\text { prospetti, schemi, orari } \\
\text { e istruzioni riguardanti } \\
\text { l'uso di attrezzature a me } \\
\text { familiari, a condizione che } \\
\text { siano brevi e chiari. }\end{array}$ & $\begin{array}{l}\text { Riesco a leggere testi } \\
\text { scritti riguardanti la } \\
\text { quotidianità del mio lavoro. } \\
\text { Riesco a comprendere } \\
\text { prospetti, schemi, orari, } \\
\text { testi burocratici semplici } \\
\text { (modulistica) e istruzioni } \\
\text { riguardanti il ritmo } \\
\text { quotidiano e settimanale di } \\
\text { lavoro, le vacanze e le ferie e } \\
\text { l'uso di attrezzature tipiche } \\
\text { del mio lavoro. Riesco a } \\
\text { capire } \\
\text { buona parte dei messaggi } \\
\text { in articoli contenenti notizie } \\
\text { sul mio settore lavorativo, } \\
\text { purché non siano troppo } \\
\text { lunghi e articolati. }\end{array}$ & $\begin{array}{l}\text { Riesco a leggere testi scritti } \\
\text { riguardanti il mio settore } \\
\text { lavorativo individuando } \\
\text { e valutando il punto } \\
\text { di vista di chi scrive. } \\
\text { Riesco a comprendere } \\
\text { prospetti, schemi, orari, } \\
\text { istruzioni riferite al mio } \\
\text { lavoro, testi di argomento } \\
\text { sociale e burocratico ad } \\
\text { esso legati (ad esempio, } \\
\text { contratti, buste paga, } \\
\text { ricevute fiscali, moduli, } \\
\text { opuscoli informativi). } \\
\text { Riesco a leggere articoli } \\
\text { e aggiornamenti su } \\
\text { macchinari e attrezzature } \\
\text { utili per il mio lavoro. }\end{array}$ \\
\hline
\end{tabular}


Benucci

2 - Comunicazione efficace in contesti interlinguistici e interculturali

\begin{tabular}{|c|c|c|c|c|c|}
\hline 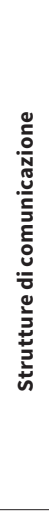 & $\begin{array}{c}\text { Analisi delle } \\
\text { strutture di } \\
\text { comunicazione }\end{array}$ & $\begin{array}{l}\text { Riesco a compiere semplici } \\
\text { operazioni di analisi e } \\
\text { trasformazione di strutture } \\
\text { morfosintattiche in testi } \\
\text { che riguardano aspetti } \\
\text { fondamentali del mio } \\
\text { settore lavorativo; conosco } \\
\text { le parole strettamente } \\
\text { necessarie per svolgere il } \\
\text { mio lavoro. }\end{array}$ & $\begin{array}{l}\text { Riesco a compiere semplici } \\
\text { operazioni di analisi e } \\
\text { trasformazione di strutture } \\
\text { morfosintattiche in testi } \\
\text { che riguardano i principali } \\
\text { aspetti del mio settore } \\
\text { lavorativo; gestisco le } \\
\text { parole essenziali riguardanti } \\
\text { il mio lavoro. }\end{array}$ & $\begin{array}{l}\text { Riesco a gestire, in molti } \\
\text { casi solo sotto forma di } \\
\text { riconoscimento, strutture } \\
\text { linguistiche (grammaticali, } \\
\text { morfosintattiche) semplici } \\
\text { in testi funzionali alla } \\
\text { comunicazione nel mio } \\
\text { i settore lavorativo. } \\
\text { Possiedo le principali } \\
\text { conoscenze lessicali } \\
\text { specialistiche riguardanti } \\
\text { il mio lavoro. } \\
\text { Gestisco frasi semplici } \\
\text { e parole per comprendere } \\
\text { testi burocratici } \\
\text { e amministrativi non } \\
\text { complessi (moduli, } \\
\text { bollettini). }\end{array}$ & $\begin{array}{l}\text { Riesco a gestire, in alcuni } \\
\text { casi solo sotto forma di } \\
\text { riconoscimento, strutture } \\
\text { linguistiche (grammaticali, } \\
\text { morfosintattiche) anche } \\
\text { complesse in testi funzionali } \\
\text { alla comunicazione } \\
\text { nel mio settore lavorativo } \\
\text { e in settori affini. } \\
\text { Conosco molte parole } \\
\text { specialistiche riguardanti } \\
\text { il mio lavoro e le strutture } \\
\text { grammaticali fondamentali } \\
\text { di manuali di istruzioni } \\
\text { professionali e per lo } \\
\text { svolgimento di attività di } \\
\text { lavoro. }\end{array}$ \\
\hline \multirow[t]{2}{*}{ 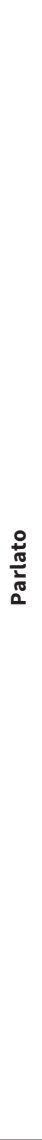 } & Interazione & $\begin{array}{l}\text { Riesco a interagire in modo } \\
\text { semplice in situazioni del } \\
\text { mio lavoro per me familiari, } \\
\text { a patto che l'interlocutore } \\
\text { parli lentamente e } \\
\text { scandisca le parole, se } \\
\text { necessario aiutandomi } \\
\text { a esprimermi. Riesco a } \\
\text { rispondere in maniera } \\
\text { essenziale a domande } \\
\text { molto semplici riguardanti il } \\
\text { mio lavoro quotidiano. }\end{array}$ & $\begin{array}{l}\text { Riesco a comunicare in } \\
\text { modo semplice in situazioni } \\
\text { tipiche del mio lavoro } \\
\text { quotidiano. Riesco a } \\
\text { seguire i punti principali di } \\
\text { conversazioni su aspetti del } \\
\text { mio lavoro a me familiari, e } \\
\text { faccio brevi interventi. }\end{array}$ & $\begin{array}{l}\text { Riesco a comunicare } \\
\text { abbastanza } \\
\text { spontaneamente in buona } \\
\text { parte delle situazioni che } \\
\text { possono presentarsi nel } \\
\text { mio lavoro quotidiano. } \\
\text { Riesco a parteciparea } \\
\text { discussioni riguardanti } \\
\text { aspetti del mio settore } \\
\text { lavorativo a me familiari, } \\
\text { a condizione che non si } \\
\text { parli troppo velocemente, } \\
\text { riconoscendo i criteri di } \\
\text { formalità (età, situazione, } \\
\text { ruolo sociale). }\end{array}$ & $\begin{array}{l}\text { Riesco a interagire in } \\
\text { maniera sciolta e spontanea } \\
\text { con colleghi e datori di } \\
\text { lavoro italiani e stranieri } \\
\text { (dialoghi, colloqui di lavoro), } \\
\text { rispettando i criteri di } \\
\text { formalità (età, situazione, } \\
\text { ruolo sociale) } \\
\text { e riconoscendo aspetti } \\
\text { come turni di parola, pause, } \\
\text { tono della voce. } \\
\text { Riesco a partecipare } \\
\text { a conversazioni } \\
\text { riguardanti il mio settore } \\
\text { lavorativo, esponendo } \\
\text { e argomentando } \\
\text { problematiche opinioni } \\
\text { e individuando soluzioni. } \\
\text { Riesco a fornire } \\
\text { informazioni tempestive } \\
\text { ai miei colleghi, anche al } \\
\text { telefono. }\end{array}$ \\
\hline & $\begin{array}{c}\text { Produzione } \\
\text { orale }\end{array}$ & $\begin{array}{l}\text { Riesco a usare le parole } \\
\text { fondamentali e alcune brevi } \\
\text { espressioni riguardanti il } \\
\text { mio lavoro quotidiano. }\end{array}$ & $\begin{array}{l}\text { Riesco a usare frasi semplici } \\
\text { ed espressioni tipiche del } \\
\text { mio lavoro quotidiano; } \\
\text { riesco a parlare in maniera } \\
\text { essenziale delle mie } \\
\text { esperienze lavorative } \\
\text { precedenti. }\end{array}$ & $\begin{array}{l}\text { Riesco a esprimermi in } \\
\text { modo abbastanza chiaro } \\
\text { su aspetti tipici del mio } \\
\text { lavoro. Riesco a esporre } \\
\text { brevemente le mie opinioni } \\
\text { e i miei bisogni (richiesta } \\
\text { di permesso o spiegazioni, } \\
\text { intenzione o capacità di } \\
\text { fare qualcosa, lamentele) e } \\
\text { parlo in modo abbastanza } \\
\text { diffuso delle mie esperienze } \\
\text { lavorative precedenti. }\end{array}$ & $\begin{array}{l}\text { Riesco a esprimermi in } \\
\text { modo chiaro su molti } \\
\text { argomenti riguardanti il mio } \\
\text { lavoro. Riesco a comunicare } \\
\text { in maniera chiara la mia } \\
\text { intenzione o capacità di fare } \\
\text { qualcosa, come anche le } \\
\text { mie lamentele o proteste. } \\
\text { Riesco a esprimere il mio } \\
\text { punto di vista su problemi } \\
\text { e novità del mio settore } \\
\text { lavorativo e a parlare delle } \\
\text { mie esperienze precedenti. } \\
\text { Riesco a comunicare al fine } \\
\text { di eseguire operazioni non } \\
\text { molto complesse di tipo } \\
\text { burocratico ed economico } \\
\text { legate al mio lavoro. }\end{array}$ \\
\hline
\end{tabular}




\begin{tabular}{|c|c|c|c|c|c|}
\hline 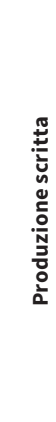 & $\begin{array}{c}\text { Produzione } \\
\text { scritta }\end{array}$ & $\begin{array}{l}\text { Riesco a scrivere brevissimi } \\
\text { testi sugli aspetti } \\
\text { fondamentali del mio lavoro } \\
\text { quotidiano, come messaggi } \\
\text { e semplici appunti. Riesco a } \\
\text { compilare brevi schede con } \\
\text { le mie generalità. }\end{array}$ & $\begin{array}{l}\text { Riesco a scrivere brevi testi } \\
\text { su aspetti familiari del mio } \\
\text { lavoro quotidiano. Riesco } \\
\text { a scrivere brevi lettere in cui } \\
\text { tratto in maniera semplice } \\
\text { aspetti fondamentali del } \\
\text { mio lavoro. }\end{array}$ & $\begin{array}{l}\text { Riesco a scrivere testi } \\
\text { semplici e chiari su } \\
\text { argomenti riguardanti } \\
\text { il mio lavoro quotidiano. } \\
\text { Riesco a riempire testi } \\
\text { di tipo burocratico } \\
\text { e amministrativo } \\
\text { non complessi } \\
\text { (moduli, bollettini). } \\
\text { Riesco a scrivere testi } \\
\text { e lettere in cui racconto } \\
\text { le mie esperienze lavorative, } \\
\text { esponendo in maniera } \\
\text { essenziale il mio punto } \\
\text { di vista. }\end{array}$ & $\begin{array}{l}\text { Riesco a scrivere testi } \\
\text { chiari e articolati su molti } \\
\text { argomenti riguardanti } \\
\text { il mio lavoro. Riesco a } \\
\text { scrivere testi (ad esempio, } \\
\text { curriculum vitae) e } \\
\text { lettere sul mio settore } \\
\text { lavorativo, esprimendo e } \\
\text { commentando il mio e altri } \\
\text { punti di vista e attenendom } \\
\text { al linguaggio burocratico } \\
\text { ed al registro formale } \\
\text { previsti. }\end{array}$ \\
\hline
\end{tabular}

Come si può osservare il Portfolio descrive nel dettaglio competenze comunicative verbali e non verbali, dando pari valore a entrambe, ed è impostato per formare una positiva rappresentazione del sé.

A livello della sociologia le rappresentazioni collettive (Dürkheim 1998) sono descritte come fenomeni culturali e sociali che danno agli esseri umani una visione funzionale del mondo e che permettono loro di vivere in una o in più realtà condivise (cf. Narcy-Combes, NarcyCombes 2019, 156): la comprensione e la descrizione delle rappresentazioni mentali sono spesso condizionate dall'orientamento filosofico di base dei differenti pensatori / ricercatori; tradizionalmente questi orientamenti si articolano attorno all'opposizione tra il dualismo e il monismo. Sul lato della condizione sociale la teoria dello spirito (Theory of Mind da cui la sigla ToM) designa l'attitudine cognitiva di attribuire stati mentali a sé stesso e ad altri e di comprenderli; essa può avere differenti accezioni tra cui anche quella di comprensione sociale ma il termine che oggi va di moda è empatia: secondo Berthoz (2015) l'empatia suppone che si sia capaci di provare emozione come questa è sentita da altri mettendosi effettivamente al loro posto, entrando in qualche modo nel loro corpo ma al tempo stesso empatia esige anche che si sia capaci di inibire questa mozione; l'empatia differisce dalla simpatia che è più distanziata.

Le rappresentazioni sociali, mentali e ToM permettono di comprendere come sono classificate e gestite le informazioni incontrate nei contatti con altri nel corso di attività: ogni avvenimento infatti riattiva le reti che sono legate all'avvenimento stesso.

I contatti linguistici dell'ansietà linguistica in contesto migratorio si complicano per via degli effetti dei differenti sistemi di valori: non sorprende che l'ansietà linguistica sia permanente nella vita quotidiana degli immigrati; gruppi sociali con maggiori insicurezze sono quelli che sono più sensibili agli effetti del prestigio rispettivo delle lingue e quelli che desiderano migliorare la loro situazione sociale, in particolare nelle classi medie inferiori e presso le donne. Le emozioni hanno un ruolo chiave nell'avviamento e nella prosecuzione 
dell'apprendimento, non sorprende che esse siano intimamente legate a ciò che si chiama comunemente motivazione. Le credenze che si possiedono su sé stessi (spesso implicite) giocano un ruolo determinante nella realizzazione di azioni da parte di chiunque, a scuola e al di fuori della scuola; è importante creare condizioni perché la motivazione si sviluppi e non misurarla. Non si devono confondere influenza e motivazione dato che la prima è un processo sociale tramite il quale un individuo chiede ad un'altra persona di cambiare il suo comportamento e/o i suoi pensieri, è dunque un processo interpersonale che può avere diverse etichette come la persuasione, il rispetto, la conformità, l'obbedienza e la leadership, mentre la motivazione è un processo interno. Invece di spingere la gente a seguire una linea di pensiero o di comportamento la motivazione dà energia necessaria per compiere un compito. Per sviluppare motivazione conviene creare e mantenere un contesto propizio, rispondere alle domande, stimolare comportamenti, valorizzare l'impegno e il modo di condursi e accompagnare gli apprendenti/interagenti; l'influenza conduce le persone a rispondere favorevolmente ad una richiesta specifica, a fare ciò che ci si aspetta da loro: motivare non significa manipolare la gente ma stimolare individui perché sviluppino la loro performance e il loro benessere.

\subsection{Problematicità della comprensione}

La ricerca svolta nell'ambito dell'intercomprensione ha riportato in primo piano le questioni relative alla comprensione dopo che per molti anni sono state considerate un riflesso obbligato e speculare, mero ricalco esecutivo, della produzione. La linguistica ha infatti sempre avvertito come problematica la comprensione lasciandola in margine ai propri interessi, considerata banale e scontata, vista come un processo che si svolge a tappe successive; anche gli studi di interlingua e delle fasi acquisizionali si sono per lo più concentrati sulla produzione. Invece le difficoltà del comprendere non riguardano soltanto performance occasionali o eccezionali ma neppure la sola realizzazione esecutiva o, per dirla con Ferdinand de Saussure, la parole; attengono invece alla competence: hanno radice sia nella forma del sistema, nella langue come sistema, sia nell'umana capacità di gestire una lingua e le sue innumerevoli parole, ossia nel language tutto intero. E proprio di questo ultimo, del language, si è tenuto poco conto in gran parte degli approcci e dei metodi per insegnare/apprendere le lingue, e per formare i formatori.

La visione lineare della comprensione linguistica cambia grazie ad una maggiore conoscenza della natura della comunicazione, agli studi di psicoanalisi, sulla memoria e sul funzionamento del cervello, a quelli sul lessico di alta frequenza e sul grado di leggibilità dei 
testi, alla diffusione dei mezzi di comunicazione - in particolare la televisione e Internet - e si fa strada l'affermazione che comprendere realmente un enunciato - e un testo - è sempre un caso di problem solving e come tale non è lineare ma è complesso e coinvolge saperi relativi sia alla langue che al language.

Anche gli studi sulla percezione grafica e uditiva hanno messo in crisi l'idea semplicistica della comprensione mostrando che le abitudini verbali pregresse, le conoscenze, le attese linguistiche e culturali, le attitudini psicologiche e le valutazioni intuitive sul cotesto pesano per favorire e orientare la ricezione visiva e uditiva. La comprensione non è avulsa dal contesto situazionale in cui ha luogo la comunicazione: la forma della frase o del testo, ricostruibile a partire dal concreto enunciato fonico o grafico che viene percepito, si rivela solo uno dei dati, il maggiore, ma non l'unico, su cui devono esercitarsi le capacità di comprensione. Dato che tali capacità non sono strettamente linguistiche, ma intellettive e cognitive, riguardano almeno due macrolivelli: la distinzione dell'espressione dei messaggi, cioè la percezione delle manifestazioni foniche / grafiche, e l'individuazione del contenuto dei messaggi, vale a dire il senso.

Non è possibile aspettarsi una ricezione prevedibile perché la ricezione si sviluppa per tentativi ed esplorazioni, ipotesi, ritorni e perché le ipotesi vengono elaborate prima ed esternamente dalle conoscenze del linguaggio verbale, su una base percettiva, prelinguistica, analizzando la situazione di produzione, il testo scritto, valutando gli interlocutori, servendosi di appigli extraformali e non verbali. Dunque se in glottodidattica è utile avere una visione pragmatica del funzionamento della comunicazione per la specifica formazione alla mediazione è indispensabile anche analizzare le strategie messe in atto da chi parla/ascolta, le relazioni che ricostruisce tra lingua e contesto, come (e se) sa ricorrere all'expectancy grammar. Proprio questo ultimo tipo di competenza, che opera sulla base della consapevolezza situazionale, della ridondanza e della conoscenza del mondo (enciclopedia), risulta molto utile per potenziare la naturale intercomprensione tra lingue. La capacità di prevedere che cosa può essere detto in un dato contesto (in base all'argomento, al luogo, al momento della comunicazione, ai ruoli, agli scopi, alla ridondanza) e in un dato cotesto (sulla base del genere comunicativo, della struttura del testo, delle norme morfosintattiche e semantiche condivise) potenziano indubbiamente il processo globale e simultaneo di comprensione. Materiali didattici fondati su questi presupposti non possono che costituire 'buone pratiche' su cui formare apprendenti di L2 ma soprattutto mediatori / interpreti perché li abituano a riflettere su tutti gli aspetti della comunicazione e a correlarli.

Vi sono poi diversi tipi di comprensione: intensiva (parola per parola), estensiva globale (skimming), mirata all'identificazione di dettagli (scanning); anche se il prodotto reale della comprensione rimane 
nel cervello dell'apprendente, e come si è detto è di non facile individuazione e valutazione, insegnare quale strategia convenga utilizzare di volta in volta e come essa funzioni è un obiettivo specifico dell'Educazione Linguistica che può essere utilmente applicato alla formazione in interpretariato. Nell'ambito della mediazione si intreccia strettamente con il non detto, il condiviso, non condiviso ecc. con la considerazione delle differenze culturali, non con la spiegazione delle differenze culturali. ${ }^{5}$

Dunque, all'interno del generale ritardo con il quale si sono sviluppati gli studi sulla comprensione, la scarsa considerazione dei processi di comprensione orale fa sì che ancora oggi si sappia molto di più sul funzionamento del codice scritto e della sua comprensione che su quello orale e sulla sua relativa comprensione. Per questo potranno costituire oggetto di interesse i risultati delle sperimentazioni non soltanto di generali pratiche di intercomprensione ma nello specifico di quelle di intercomprensione all'orale.

I meccanismi comunicativi dell'oralità, canale nel quale avviene al maggior parte delle azioni di mediazione, al di là delle specificità delle singole lingue e culture condividono alcune caratteristiche, legate alla testualità, al canale comunicativo ecc.: per esempio si sa che in tutte le lingue la produzione orale risente di alcuni fenomeni indotti necessariamente dal canale utilizzato come la frammentarietà, il carattere perituro e la non permanenza dei segni, la funzionalità dei fenomeni di ritmo e prosodia, il ricorso a segnali discorsivi e ad aspetti paralinguistici ma anche dei rapporti tra gli interlocutori e dei riti di interazione. Quanti corsi di lingue o insegnanti tengono conto di questi aspetti trasversali di conoscenza? E quanti mediatori possiedono una sensibilità sviluppata e cosciente di tali problemi? Pochi. In sperimentazioni didattiche condotte di recente si è rilevata tutt'ora una scarsa introduzione di procedure che facciano esplicitare agli apprendenti i processi attivati durante l'ascolto e una quasi totale assenza di azioni che svolgano una verifica della adeguatezza delle strategie adottate.

Gli studi sui processi di intercomprensione e sul costruttivismo sociale e culturale, ancora scarsamente applicati in concreto, rappresentano una utile cornice di riferimento di cui tenere conto per ottenere una comunicazione efficace poiché ci dicono che la conoscenza di una lingua, anche la lingua materna, è sempre parziale e che occorre valorizzare le singole competenze e abilità parziali possedute dall'individuo. Ma affermano anche che tutte le conoscenze parziali sono meno parziali di quanto sembri poiché chi ha già appreso una lingua conosce già molte altre cose di altre lingue anche senza ren-

5 Le associazioni per interpreti sono diventate nel tempo associazioni per mediatori tanto che, in molti casi, le due professioni si sovrappongono e integrano anche se occorrerebbe un chiarimento pragmatico. 
dersene conto grazie alla capacità di transfert tra aspetti linguistici, culturali, pragmatici, testuali ecc. da una o più lingue conosciute ad altre lingue sfruttando le similarità all'interno di famiglie linguistiche e anche tra lingue tipologicamente lontane. Dunque i presupposti dell'intercomprensione possono essere coniugati con quelli del CLIL, già esposti in $\S 2.1$.

Altri principi dell'intercomprensione possono utilmente essere adottati in ambito di formazione di adulti immigrati: l'apprendimento è un processo attivo in cui si impara ad imparare mentre si impara; si impara solo appoggiandosi a strutture di conoscenza pregresse; la costruzione di conoscenza è un processo mentale con attività che coinvolgono altre facoltà e i sensi; il linguaggio usato influenza l'apprendimento; l'apprendimento è un'attività sociale; si impara in relazione alle proprie conoscenze e credenze e alla consapevolezza del ruolo che gioca la consapevolezza di tali conoscenze; la motivazione non solo favorisce l'apprendimento ma ne è componente essenziale; la dimensione pragmatica della comunicazione ha un grande valore così come lo sviluppo di attitudini affettive positive (emozioni, sentimenti, motivazione). È chiaro il forte impatto psicologico di tali principi epistemologici.

Per ottenere una buona comunicazione bisognerebbe che entrambi i parlanti (gli autoctoni e gli immigrati) imparassero a far leva sull'insieme delle loro competenze, sia culturali sia linguistiche, acquisite in ambito istituzionale o personale (musica, viaggi, conoscenze ecc.) e a costruirsi i rudimenti di una 'grammatica della comprensione e/o della produzione' individuando i punti di convergenza translinguistica (regole di passaggio) e i 'tranelli' linguistici da evitare (regole di vigilanza) attivando le competenze pregresse (non solo lessicali), richiamando conoscenze di domini e al loro interno di situazioni comunicative (luoghi, persone, spazi, esperienze di contatto, conoscenze specializzate, elementi paralinguistici, aspetti non verbali ecc.). Si tratta di rendere coscienti i locutori di ciò che sanno fare con la/e lingua/e e del potere che essa/e ha/hanno e di abituarli a mobilitare il proprio potenziale cognitivo e interattivo.

L'incremento in questi ultimi decenni dell'impiego della lingua orale e l'uso sempre meno frequente della comunicazione scritta tradizionale ha portato a riconsiderare con maggior accuratezza scientifica i problemi dell'ascolto e ad approfondire i meccanismi psicolinguistici della comprensione all'ascolto cercando di indagare sui meccanismi mentali di elaborazione del messaggio orale. Si tratta di un processo attivo in cui almeno tre elementi fondamentali interagiscono: le conoscenze dell'ascoltatore (la conoscenza del modo, quella dell'argomento ma anche delle regole di interazione), il contesto situazionale (identità dei parlanti, loro intenzioni e luogo/tempo dell'interazione) e il testo linguistico (tipologia del testo ma anche le tradizionali componenti come lessico, grammatica ecc.), in cui 
sono implicati vari processi cognitivi come l'inferenza, la produzione e verifica di ipotesi, il completamento ecc.

Il livello concettuale pone in evidenza il ruolo della struttura del messaggio nel determinare modalità diverse di decodificazione e memorizzazione (Lafon 1969) come è il caso noto che nella decodificazione l'ascoltatore abbia maggiori difficoltà se la struttura del discorso è in contrasto con le sue aspettative linguistiche facendo cadere le strategie interpretative che sono generate dalle sue conoscenze pregresse e dalle aspettative: vi sono dunque fattori di ordine linguistico e psicologico che operano congiuntamente nel processare il messaggio ma anche fenomeni di organizzazione percettiva degli stimoli verbali (cf. i primi studi di Osgood, Sebeok [1965] e Carroll [1964]). Secondo il modello globale della comprensione orale di Carroll (1964, 58-61), vi sono alcune tappe nel processo di comprensione: compensazione di deficienze di contesto con ricorso a ridondanza delle strutture linguistiche; analisi e sintesi derivanti dal ritmo di emissione; capacità ricettiva del destinatario in base alla sua conoscenza della lingua L2 e al suo livello di maturazione metacognitiva; conoscenza della natura del codice e delle regole pragmatiche relative a una determinata situazione; identificazione simultanea e combinata delle precedenti condizioni. Dunque si tratta di operare una sintesi anticipatoria, una analisi e una sintesi conclusiva, secondo le condizioni già da tempo indicate da Titone (1971, 88-90): distintività dei singoli componenti e concatenazione degli elementi nel tutto. I parlanti non nativi presentano difficoltà anche perché in loro diminuisce l'uso adeguato degli indizi di tipo semantico a favore di una maggiore attenzione alle informazioni derivanti dagli elementi sintattici e generalmente strutturali del codice a detrimento di quelle di natura pragmatica e extralinguistica. Il dibattito su questi aspetti si è sviluppato negli anni a partire dai risultati della Scuola di Oslo e dagli studi di Rommetveit (1974) ma la natura complessa del problema resta ancora da esplorare in molte sue componenti e variabili.

\subsection{Comunicazione interculturale}

Fare Educazione Linguistica oggi significa non poter prescindere da una forte posizione politica nel senso di attenzione costante ai fenomeni sociali e alle problematiche comunicative odierne. La comunicazione interculturale e l'integrazione sociale sono i fondamenti della mediazione interculturale, è quindi essenziale che la formazione per mediatori culturali nell'ambito dell'immigrazione si ispiri ai principi dell'apprendimento interculturale con adeguata metodologia. Tali principi riguardano il rispetto dell'identità culturale, le conoscenze culturali e le abilità necessarie per assumere comportamenti adegua- 
ti ad una partecipazione attiva e piena nella società nel rispetto degli altri individui, delle altre culture, dei gruppi etnici e delle religioni.

La formazione interculturale deve essere fondata innanzi tutto sulla coscienza delle profonde modificazioni dei tessuti sociali e della natura delle migrazioni ma anche dei mutamenti della scrittura delle lingue, delle loro manifestazioni orali e trasmesse, dei livelli di formalità e dei concetti di adeguatezza e correttezza: secondo una idea di cultura strettamente legata a quella di lingua. Va inquadrata all'intersezione degli interessi di linguisti, glottodidatti e sociologi riflettendo congiuntamente su lingue, culture, apprendimento, formazione, politiche linguistiche e società perché un'Educazione Linguistica che ignori la realtà non solo non svolge il suo ruolo in termini di benefici a cui può condurre in termini socio-culturali, ma rischia di risultare pure eticamente inadeguata e quindi oltre che inutile, palesemente contestabile (Santipolo 2018a, 11).

Tuttavia, come ricorda Trotta:

quando si parla di interculturalità spesso non si tiene conto delle varie accezioni che questo termine può assumere a seconda di chi lo ascolta o di chi lo pronuncia o scrive. 'Interculturale' può essere l'ambiente' [...], ma anche la 'comunicazione', il 'contatto', l'esperienza', l'educazione', la 'pedagogia' e non ultima la 'competenza'. Qualsiasi collocazione si dia a questo aggettivo, esso presupporrà sempre che ci siano almeno due realtà 'culturali' che entrano in contatto tra loro (come suggerito, appunto, dal prefisso inter-) stabilendo dei rapporti di forza. $(2021,57)$

La formazione alla comunicazione interculturale dovrebbe di preferenza svolgersi in ambienti multiculturali al fine di favorire il decentramento dalla cultura di appartenenza e il confronto costruttivo, così come la sensibilizzazione verso il potere delle parole e dei diversi linguaggi. L'esplicitazione dei vissuti di ciascuno, la riflessione sulle conoscenze, sui principi di divisione, sui valori, sulle tassonomie, sui tabù può essere facilitata da specifiche tecniche come lo story-telling, le varie realizzazioni del role play e tutte le attività volte a sviluppare la comprensione purché ispirate al principio della partecipazione attiva, costruttiva, contestualizzata e condivisa.

La riflessione interculturale deve avere per oggetto sia la cultura del Paese ospitante che quella dei Paesi di provenienza dei mediatori (se sono migranti) e quelli di probabile provenienza di coloro verso i quali si rivolgerà la loro azione professionale

Altro aspetto importante è quello dell'acquisizione di competenze utili per evitare che insorgano, per risolvere e mediare i conflitti culturali. Uno degli ambiti più esplorati è quello sanitario. Dato che nel rapporto medico/infermiere-paziente, la disparità di ruoli e di saperi e le diverse competenze linguistiche possono creare incomprensio- 
ni e contrarietà, possono quindi compromettere la comunicazione e soprattutto gli effetti della cura. Si è accennato nel capitolo 1 al problema dell'insicurezza linguistica: in tale contesto essa viene accentuata dalle condizioni di fragilità fisica e psicoemotiva, e dall'assenza di condivisione di un comune codice linguistico e di valori culturali condivisi. Se necessario, oltre all'attività di mediazione culturale e all'uso della lingua d'origine, si potrà ricorrere alla L2. Il buon mediatore interculturale non agisce come esperto di cultura ma si accontenta di facilitare l'espressione dell'utente senza attribuire significati e spiegazioni o negoziare le proprie conoscenze con quelle dell'interessato; non impone propri definizioni e saperi e usa strategie comunicative di accomodamento. Proprio le strategie di accomodamento sono costituite anche da elementi extralinguistici (significato della gestualità e di altri linguaggi non verbali come l'uso dello sguardo, della cronemica ecc.) che, come si è visto, sono fortemente improntati alle culture di riferimento, o paralinguistici (tono elevato o sommesso per creare enfasi, per chiarire o contestualizzare elementi che si prestano a malintesi ecc.).

Il mediatore / interprete deve riflettere sulla propria appartenenza culturale oltre a conoscere i tratti più salienti di quella dell'utente (in particolare i tabù) e la propria professione, possedere una buona capacità di ascolto, essere paziente e disponibile ma anche conoscere i riferimenti del contesto situazionale e culturale. Il mediatore per facilitare pari opportunità deve dunque possedere un competenza comunicativa interculturale, deve mantenere una «giusta distanza» (Balboni, Caon 2015), sintetizzata in cinque saperi da M. Byram (1999) (cf. Benucci, Grosso 2015, 74): saper essere (attitudine), relativizzare sé stesso e accettare l'altro mettendo da parte i propri presupposti e valori decentrandosi; sapere come ciascuno è visto dall'altro (regard croisé); saper comprendere (interpretare e mettere in relazione) documenti, testi, situazioni, atteggiamenti e 'oggetti' dell'altro; saper scoprire e apprendere nuovi comportamenti, valori e credenze; saper interagire in tempo reale sulla base delle competenze dell'altro e delle esigenze della situazione; saper assumere responsabilità ricorrendo ad una 'competenza culturale critica'.

Le teorie sull'apprendimento e sulla formazione interculturale fanno riferimento ad alcuni modelli teorici, tra i quali i più impiegati e conosciuti sono:

- il Modello Dinamico di Sviluppo della Sensibilità Interculturale di Bennett (1993), di natura sociologica, comprende sei fasi dello sviluppo della sensibilità interculturale raggruppate nelle due macrofasi etnocentrica e etnorelativa: si passa dalla fase della negazione a quelle della difesa, della minimizzazione, accettazione, adattamento e infine dell'integrazione; si è tenuto conto dei presupposti di queste tassonomie ma sono troppo schematiche per gli scopi della formazione del mediatore; 
- il Modello multimodale di Byram (1997) è maggiormente orientato all'insegnamento, secondo la verifica delle dimensioni culturali di cinque fattori che connotano un buon intercultural speaker: attitudine, conoscenza, abilità di interpretare e mettere in relazione, abilità di scoprire e interagire, consapevolezza culturale critico - valutativa; è stato in parte adottato per le riflessioni di questo paragrafo perché pone particolare attenzione alla comunicazione non verbale e al suo ruolo nella didattica interculturale;

- il Modello di Competenza Comunicativa Interculturale di Balboni (1999) affronta l'interculturalità nella prospettiva della performance, delle abilità relazionali e delle problematiche che possono presentarsi in una situazione comunicativa interculturale; è un modello molto impiegato per la didattica delle lingue moderne ma che ben si adatta anche alla formazione del mediatore / interprete.

Tutti questi modelli partono comunque dal presupposto che la competenza comunicativa interculturale si costruisce tramite un processo graduale di apertura e di integrazione della propria visione culturale a quella di altri modelli culturali essenzialmente attraverso la conoscenza, il superamento di pregiudizi e di stereotipi, il rispetto dell'altro e delle differenze.

\subsection{Mentori e mediatori: la mediazione linguistico-culturale}

In molti Paesi europei, e anche in Italia, i fenomeni di comunicazione e di confronto con differenti sistemi linguistico-culturali immessisi di recente nelle comunità autoctone hanno prodotto variazioni importanti e hanno contribuito a far evolvere lo spazio linguistico tradizionale dando luogo ad un «sistema quadripolare» (Vedovelli 2013), comprendente anche le varietà interlinguistiche di apprendimento/ acquisizione degli immigrati, le loro lingue di origine, varietà interculturali formatesi dal contatto di culture anche molto lontane tra di loro, grazie alla nascita di comunità 'ibride' caratterizzate da permeabilità e scambi culturali e linguistici (Bagna et al. 2003; 2004) che si manifestano nei vari aspetti di vita quotidiana, nei gruppi pienamente inseriti nelle società di arrivo ma anche in quelli situati al loro margine o da esse esclusi, finanche nel contesto penitenziario.

I soggetti migranti si trovano ad avere difficoltà a livello psicologico (vedi cap. 4 di Monaci in questo volume) causate dall'estenuante viaggio per raggiungere il Paese ospitante o dalle condizioni vissute nel Paese di origine: va ricordato che non tutti gli immigrati lasciano i loro Paesi per motivi economici, alcuni di essi fuggono da guerre e persecuzioni. Le principali e immediate difficoltà con le quali 
si interfacciano sono quella di non sapere come comunicare i propri bisogni a causa della non conoscenza della lingua del Paese di arrivo e la convivenza forzata nei centri d'accoglienza con altre persone, ciascuna con il proprio bagaglio linguistico-culturale, in cui si crea un contesto di 'superdiversità'. Oltre ai centri accoglienza, un altro esempio di superdiversità, e di forzata convivenza, è il contesto penitenziario in cui le presenze straniere sono numericamente elevate e vi sono non indifferenti problemi di comunicazione, sia tra i detenuti stessi sia tra di essi e il personale penitenziario. Infatti il profilo del detenuto immigrato è singolare poiché, oltre a vivere la condanna lontano dal Paese di origine, probabilmente se non fosse straniero non sarebbe recluso, ${ }^{6}$ e si trova ad essere 'straniero' tra gli 'stranieri' della società, 'diverso' tra i 'diversi'.

Prima tappa al processo di integrazione dell'immigrato è la lingua, intendendo tuttavia il termine 'integrazione' non come un'assimilazione della cultura e della lingua del Paese di arrivo, poiché in una ottica interculturale l'immigrato non deve fondersi con la società ospitante ma ha il diritto di rispettare i propri usi e costumi d'origine. Deve esse favorita una coesistenza tra la propria lingua madre (L1), l'identità e le radici, e la lingua acquisita (L2), della nuova vita; perché ciò avvenga è importante l'opera del mediatore linguistico-culturale.

Da anni si parla ormai della figura del mediatore interculturale, figura spesso richiamata nelle politiche di accoglienza degli immigrati ma che continua ad avere uno statuto debole la cui definizione è tuttora incerta a livello europeo: come dimostrano Casadei e Franceschetti (2009) analizzando diversi Paesi europei, vi sono differenze e analogie sia nella concezione del dispositivo, che nella formazione e reclutamento, nonché nel livello di normazione e di risorse dedicate. In alcuni Paesi e situazioni la mediazione coincide con l'azione di una figura terza mentre in altri è assunta da operatori sociali; in alcuni casi coincide con quella dell'interprete / traduttore. Le differenze sono labili: il traduttore è concentrato quasi unicamente sul messaggio, sulla lingua, il mediatore linguistico-culturale ha una formazione che va oltre la conoscenza linguistica perché comprende anche la conoscenza della cultura del Paese di origine del migrante e la cultura del Paese ospitante, una competenza semiotica, la capacità di gestire il contesto e di negoziare tra almeno due parti (Siebetcheu 2011, 13), la gestione della sfera degli atteggiamenti psicologici ed emotivi. Deve conoscere i principi legislativi del diritto internazionale comunitario e nazionale sulla tutela dei diritti umani, gli ordinamenti e i regolamenti dei settori in cui opera, l'organizzazione di istituti

6 Si è insistito più volte sulla doppia marginalizzazione e estraneità del detenuto straniero, cf. almeno Benucci 2015 e Bormioli 2017. 
e di servizi, avere padronanza di tecniche di mediazione culturale e possedere nozioni di principi fondamentali di pedagogia interculturale e psicologia dell'immigrazione.

Vi è convergenza rispetto ad alcune competenze importanti di tale figura: la mediazione linguistico-culturale assume come punto di partenza l'analisi dei bisogni, delle motivazioni, delle caratteristiche e delle risorse degli attori coinvolti: si differenzia in base alla tipologia di persone a cui è rivolta, dai più generici 'immigrati' ai rifugiati e richiedenti asilo. L'accoglienza di rifugiati e richiedenti asilo e il ruolo dei mediatori interculturali sono determinati anche dalle politiche dei Paesi di accoglienza, motivo per il quale non è semplice fare delle generalizzazioni.

Le motivazioni di coloro che emigrano, che vanno da quella meramente strumentale e integrativa a quella intrinseca, dipendono dai tipi di bisogni, dalla necessità di assistenza, dalla natura della progettualità che si costruisce intorno a loro. Essi si trovano in spazi nuovi e devono interagire con operatori, frequentare alcune attività proposte, inserirsi nel mondo del lavoro nella società d'accoglienza. Pur dovendo anche apprendere la lingua la loro formazione linguistica non sempre è adeguata e non tutti si sentono obbligati a frequentare i corsi di L2: alcuni lo fanno solo per ricevere protezione, tutele e il pocket money, altri si impegnano con costanza dietro alla motivazione di migliorare la propria vita e per avere nuove opportunità nel Paese di accoglienza; ma altri ancora, anche se in minor percentuale, non si applicano. Anche per l'apprendimento della L2 l'intervento del mediatore può risultare utile, sia come facilitatore linguistico sia come motivatore ma soprattutto come persona che dimostra interesse e rispetto verso la cultura, le lingue e i linguaggi degli altri.

Dal momento che non è sufficiente riportare il solo contenuto verbale di un messaggio perché ci sia una effettiva attività di mediazione, il mediatore oltre a conoscere la realtà di provenienza del soggetto migrante deve essere in grado di creare un ambiente favorevole all'interazione, collaborativo e rispettoso dell'altro senza prevaricarlo o tentare di persuaderlo, deve inoltre ottenere il consenso al colloquio da parte del soggetto.

Un mediatore professionista sa relativizzare le culture e valorizzarle, ne conosce i valori ma non li giudica, sa mantenere una certa distanza emozionale, sa ascoltare attivamente ed empaticamente, cioè, secondo Tewolde Berhe, ${ }^{7}$ sa «prestare attenzione al messaggio che l'altro vuole trasmettere e non solo meramente alle parole che comunica» e svolge attività di collegamento tra le parti interessate.

7 Mediatore che ha tenuto un intervento al ciclo di seminari formativi Mentorship, mediazione e inclusione organizzato dall’Università per Stranieri di Siena nell'ambito progetto COMMIT dall'8 febbraio al 12 marzo 2021. 
La figura del mediatore è (o dovrebbe essere) presente in vari ambiti della società, da quello sanitario a quello scolastico a quello sociale, giuridico-amministrativo, del mondo del lavoro e della sua tutela: per ogni ambito sono richieste competenze specifiche di interazione ma restano gli stessi i principi etici di comportamento, le competenze sottostanti all'attività di mediazione e la professionalità che deve potersi esprimere a pieno nei diversi contesti di interazione e al contempo aggiornarsi, senza stereotipizzazioni e pregiudizi. Tra le conoscenze del mediatore che si trovano costantemente indicate, almeno a livello europeo, al primo posto si trova quella della legislazione e delle istituzioni seguita dalla capacità di ascolto attivo, risoluzione dei problemi, flessibilità, capacità di cooperare, affidabilità ma viene anche segnalata la competenza nel gestire le pressioni derivate dai bisogni posti dai migranti e quelle legate al contesto giuridico e di welfare dei servizi di accoglienza e integrazione.

In ambito socio-sanitario oltre alle prime attività di accoglienza emergenziali, per il mediatore è utile superare le barriere culturali e facilitare la comprensione tra le due parti: il dialogo tra il medico e il paziente è molto delicato e deve essere per quanto possibile empatico (a questo è stata dedicata gran parte della ricerca di Daniela Zorzi).

Nelle scuole il mediatore è di supporto sia per l'alunno straniero che deve entrare a far parte di una classe già formata sia per la famiglia immigrata: l'iscrizione a scuola del figlio spesso è tra le prime azioni istituzionali degli immigrati adulti, per questa ragione il mediatore deve far comprendere loro il nuovo ambiente in cui si trovano, ma deve soprattutto evitare misunderstanding. Svolge però anche attività di supporto all'insegnante per creare attività interculturali.

Nel contesto penitenziario, il mediatore è una figura fondamentale, ma più che in altri contesti poco presente, per le interazioni di natura giuridica e quotidiana con gli operatori, si adopera per creare un clima di convivenza tra le varie etnie, cercando di farle conoscere e rispettare, si occupa di offrire una panoramica dei diritti di cui il detenuto straniero non è a conoscenza o che non comprende,

In ambito lavorativo aiuta i migranti ad inserirsi nel mondo del lavoro, offrendo servizi di consulenza: conoscere la situazione di provenienza del soggetto immigrato lo aiuta a redigere il CV, a cercare l'impiego per lui più adatto; svolge un ruolo di informatore sui diritti sindacali, di aiuto nel disbrigo delle pratiche burocratiche.

Il mediatore deve quindi avere una sensibilità interculturale molto elevata, possedere rudimenti di tecniche di comunicazione e traduzione ma ha anche necessità di conoscere il funzionamento amministrativo-burocratico del Paese di accoglienza, essere costantemente informato sulle modificazioni del tessuto sociale in cui aspirano ad inserirsi gli immigrati. Deve ricordare che il suo ruolo non è quello di rappresentanza della comunità di cui è originario, il rischio più frequente è infatti che egli non abbia il necessario distacco nei con- 
fronti della propria cultura che non si limiti a un lavoro di decodifica dei comportamenti culturali delle due culture, di accoglienza e di provenienza. Potrà trovarsi anche in situazioni conflittuali, soprattutto per aspetti legati alle religioni, e non sarà facile mantenere il distacco necessario e la capacità di analisi scevra dall'influenza dei valori di appartenenza.

Dal punto di vista più propriamente tecnico dovrà adottare le modalità della comunicazione efficace:

- riformulare e chiarire concetti poco chiari o incompresi;

- se necessario chiarire a sé stesso gli scopi dei messaggi, le intenzioni degli interlocutori;

- essere paziente soprattutto quando gli interlocutori mostrano difficoltà comunicative;

- avere coscienza di cosa sono in grado di comunicarsi gli interlocutori;

- ricorrere, e interpretare, alla comunicazione non verbale e se necessario spiegarla agli interlocutori;

- saper leggere e interpretare gli impliciti e gli stereotipi e aiutare gli altri a relativizzarli per superare conflitti;

- saper ricorrere se necessario a strategie di accomodamento come la semplificazione.

Concorrono alla sua formazione varie discipline che vanno almeno da quelle giuridiche (quadro normativo sull'immigrazione e sui diritti dei migranti nel Paese ospitante), a quelle storiche (elementi di storia dell'immigrazione), a quelle linguistiche (sistemi linguistici di riferimento, teorie e tecniche di interpretariato, teorie della comunicazione), a quelle psicologiche (conoscenza dei meccanismi di esclusione, discriminazione, risoluzione dei conflitti), sociologiche (teoria di genere, comunicazione istituzionale) ed etno-antropologiche (differenze socio-culturali, teorie sull'intercultura). Inoltre, in base agli specifici campi di intervento, dovrà essere competente in quelli della salute, della formazione (soprattutto scolastica), dei servizi svolti presso gli uffici di polizia e gli uffici legali, dei servizi degli uffici pubblici, dei settori lavorativi di maggior interesse per gli immigrati, informatico. E dovrà anche adattare il suo operato alle normative del Paese in cui si trova a svolgere la sua professione.

Le narrazioni dei mediatori linguistici [...] raccontano di una visione della mediazione che rimanda alla prospettiva gramsciana sulla traduzione intesa come prassi sociale che comporta un lavoro attraverso i confini linguistici e richiede una consapevolezza dell'interazione fra forze economiche, culturali e politiche sottostanti alla produzione di significato in ogni società e non solo nel momento di contatto tra due lingue. Per queste ragioni, le narrazioni in oggetto possono essere concepite come storie 'in contrap- 
punto' per lo stesso senso traslato che lo storico della cultura Said (1993) ha mutuato dalla storia della musica e poi diffuso nelle sue opere. In particolare, le narrazioni dei mediatori [...] richiamano il senso contrappuntistico per l'andamento polifonico con cui si generano, si diffondono e s'intrecciano in luoghi dove il potere di nominare e dare legittimazione alla loro pratica è limitato dai rigidi protocolli istituzionalizzati e dettati dalle politiche migratorie. (Taronna 2015, 167)

Sono criticità note della figura professionale del mediatore il problema dei confini di ruolo rispetto ad altre figure professionali o agli utenti, la responsabilità dell'intervento soprattutto in situazioni delicate, la definizione di un'etica professionale e i problemi legati alla precarietà delle condizioni di lavoro, così come l'esigenza di una maggiore professionalità.

In alcuni Paesi europei, come l'Italia, nei centri di accoglienza per richiedenti asilo è prevista la figura dell'insegnante di italiano, spesso però non presente o con competenze improvvisate che non sono all'altezza di quelle di analoghe figure operanti in altri settori della società o dell'educazione. La figura di insegnante di italiano L2 nei centri di accoglienza è sottovalutata, in realtà essa ha bisogno di un'adeguata formazione e di una particolare sensibilità, tanto che spesso il suo ruolo viene assunto dal mediatore, per questo si insite qui su competenze che quest'ultimo deve possedere e che vanno al di là di quelle tradizionalmente attribuite/richieste a questa figura. Ma quando l'insegnante di italiano è invece presente il più delle volte deve divenire a sua volta lui stesso un mediatore.

Può aprire nuove prospettive di visione una riflessione sui concetti di traduzione e mediazione: il traduttore di testi scritti necessita di performance molto precise e tecniche dal punto di vista soprattutto del codice lingua, del testo e delle pratiche e tecniche traduttive, il mediatore / interprete rivolge la propria attività soprattutto a testi orali e la accompagna con aspetti legati alla gestione delle diverse personalità, al dialogo interculturale, di socializzazione. $\mathrm{Ma}$ entrambi devono conoscere bene i due codici coinvolti, le culture di riferimento, le informazioni sui temi trattati, saper tradurre e interpretare i significati delle informazioni.

Per il mediatore è importante la conoscenza dei contesti di partenza dei migranti, soprattutto riguardo all'aggiornamento sulle crisi e sui problemi che stanno attraversando i Paesi di provenienza: una questione delicata che dovrebbe essere affrontata prima di tutte le altre nell'ambito della sua formazione è quella dell'asimmetria di potere e della discrezionalità del suo agire, che possono essere correttamente gestite solo se viene sviluppata una consapevolezza rispetto al proprio posizionamento, unitamente al rischio di burn-out che nel lavoro con i rifugiati si acuisce. Infine dovrebbe essere capace 
di accompagnare i percorsi individuali dei migranti ma anche di lavorare sulle comunità ospitanti, in modo da creare terreni favorevoli all'inclusione sociale, al contrasto degli stereotipi e dei pregiudizi, infine al superamento della necessità del suo operato.

A conclusione di queste riflessioni sul ruolo e sulle competenze del mediatore si propone un modello di intervista elaborato per rilevare le opinioni sul programma di mentorship ideato e realizzato nell'ambito del progetto COMMIT per accompagnare le famiglie di rifugiati in un percorso di inserimento nel Paese di accoglienza.

\section{Glossario}

mentor

cittadino della comunità di arrivo che supporta le famiglie rifugiate nell'inserimento nella società di arrivo

mentorship relazione che si instaura tra il mentor e i rifugiati con i quali viene messo in contatto

mentee famiglia o membro di una famiglia rifugiata coinvolto nel processo di mentorship

comunità/ luogo in cui si stabiliscono i rifugiati

Paese di arrivo

comunità/ luogo da cui provengono i rifugiati

Paese di partenza

1. Il programma di reinsediamento di rifugiati e richiedenti asilo ha fornito informazioni chiare e complete sul ruolo del mentor e della mentorship?

2. In quali ambienti sono state svolte le attività di mentoring?

3. Quali strumenti sono stati utilizzati per le attività con i mentee? Specificare.

4. Quali attività che sono state realizzate durante il corso? Specificare.

5. Quali sono stati gli aspetti della personalità, delle conoscenze ed esperienze di vita più utili all'attività di mentor? Specificare con esempi di situazioni concrete.

6. È stato necessario il supporto di altre persone per riuscire ad aiutare i mentee? Specificare con esempi di situazioni concrete.

7. Che rapporto ha stabilito con i mentee?

8. Sono sorte difficoltà legate a differenze culturali? Se sì, quali? Come sono state risolte?

9. Ci sono state difficoltà legate alla comprensione/produzione della lingua dei mentee? Quali?

10. Il rapporto con i mentee ha permesso di imparare qualcosa di più sulla loro lingua e/o cultura? Specificare.

11. Si sono stabiliti contatti, o creati rapporti stabili tra suoi amici/ conoscenti e i mentee?

12. Ha ricevuto domande, richieste di chiarimento da parte dei membri della Sua comunità sul programma di mentorship e sui mentee? Se sì quali?

13. È stata un'esperienza di crescita? Se sì, in che modo?

14. Quali sono le qualità di un buon mentor?

15. Quali sono i più rilevanti benefici dell'esperienza fatta di mentorship?

16. Come potrebbe essere migliorata in futuro l'attività di mentoring?

17. Quali strumenti possono essere utili per future attività di trainer? 
Per l'ambito specifico della mediazione in carcere si propongono invece alcune domande di (auto)valutazione che mirano a verificare la sensibilità e la professionalità riguardo a certi temi/concetti 'caldi' come la gestione: delle coordinate culturali di tempo e spazio, di stereotipi, pregiudizi, impliciti, tabù; delle funzioni della mediazione di prevenzione per preparare a vivere e ad agire nelle società multiculturali; di aiuto per preparare ad affrontarne le sfide; di recupero per preparare ad aiutare la risoluzione dei problemi che nascono dai conflitti presenti nelle società; delle relazioni come il clima all'interno del gruppo dei pari, la costruzione della conoscenza attraverso il dialogo e la relazione con gli altri, livello di collaborazione e cooperazione.

Data la natura dell'ambiente carcere particolare attenzione dovrà essere posta alla gestione dei conflitti mettendo a fuoco gli interessi, non le posizioni; cercando di capire come l'individuo percepisce le posizioni o le rivendicazioni dell'interlocutore e cosa gli impedisce di accettarle e perché oppone resistenza. Quali strategie si possono adottare se capita che valori umani quali il senso di sicurezza, l'identità individuale, il riconoscimento sociale e culturale ecc. siano minacciati: si osservano le 'cornici' e si ipotizzano variazioni possibili e alternative ai propri frame, mettendosi dalla parte dell'‘altro' senza decidere a priori cosa è più rilevante, si prende tempo usando parafrasi ecc.

1. Conoscere la lingua materna del detenuto è sufficiente per essere un buon mediatore? Perché?

2. Quali sono le principali coordinate culturali che possono minare un buon rapporto di mediazione?

3. Quali segni non verbali possono essere la spia di un processo di radicalizzazione?

4. Quali sono le principali funzioni della mediazione in carcere?

5. La comunicazione è influenzata da fattori di differenza personali, socioculturali, relazionali, degli spazi fisici e simbolici in cui avviene. Proporre qualche esempio

6. I linguaggi non verbali comprendono: cinesica, prossemica, vestemica, cronemica, aptica. Quali di essi devono essere oggetto di particolare attenzione per una buona mediazione in carcere?

7. Riguardo alla gestione dei conflitti, di fronte ad un atteggiamento difensivo-aggressivo quali domande occorre porsi e quali strategie si possono adottare? 



\section{Consapevolezza interculturale e interlinguistica: riflettere sulle lingue e culture dei beneficiari}

Giulia I. Grosso

Università degli Studi di Cagliari, Italia

Sommario 3.1 Il concetto di consapevolezza interculturale e interlinguistica. 3.2 Osservare le lingue e le culture. - 3.3 Corpora e didattica.

\subsection{Il concetto di consapevolezza interculturale e interlinguistica}

In questo capitolo si cercherà di mettere a fuoco il concetto di consapevolezza interculturale e interlinguistica in relazione ai profili di tutti gli attori che popolano le società europee in qualità di 'società di arrivo' e alle interazioni che all'interno di esse avvengono, coinvolgendo cittadini, docenti, mediatori, operatori dell'accoglienza, immigrati neoarrivati e nello specifico i cosiddetti refugee background students.

Partendo da una visione che intende analizzare le reciproche relazioni in termini di consapevolezza interlinguistica e interculturale si delineerà:

- quale potenziale linguistico, culturale e quali relazioni di reciproco arricchimento possono dispiegarsi in società plurilingui e pluriculturali;

- in che modo è possibile incentivare sia nei contesti didattici che al di fuori di essi questi circoli virtuosi positivi. 
Si cercherà infine di offrire una prospettiva didattica sui dati offerti dai corpora per osservare gli aspetti interlinguistici e interculturali.

Recentemente, il concetto di consapevolezza è stato ripreso e analizzato da parte di più studiosi (Santipolo 2018a), specie in ambito glottodidattico, in particolare con le specificazioni di Balboni (1999), a partire dal movimento della Language Awareness, nato negli anni Ottanta, in merito alla consapevolezza linguistica e a come essa sia legata al concetto di riflessione sulla lingua. Secondo Garret e James (in Santipolo 2018a, 8), essa corrisponde a una «explicit knowledge about language and conscious perception and sensitivity in language learning, language teaching and language use», una conoscenza esplicita della propria capacità di riflettere sulla lingua che viene quindi raggiunta e impiegata sia in contesti didattici (language learning and teaching) che negli usi linguistici quotidiani.

Come chiariscono gli stessi autori, l'incremento di tale consapevolezza, che abbraccia gli aspetti linguistici e culturali, ha quindi dei risvolti di natura sia teorica che pratica, tanto in ambito glottodidattico quanto per determinare scelte di politica linguistica. In particolare, a livello glottodidattico, molti sono i contributi che riportano ricerche condotte sul legame tra consapevolezza e competenza grammaticale: è doveroso in primis ricordare il contributo di Tullio De Mauro su questo tema, così come i contributi più o meno recenti (Bialystok 1986; 2001; Peppoloni 2018), che inducono a considerare le potenzialità contenute nella capacità, da parte di un individuo, di osservare i processi e gli usi linguistici, e quando necessario, selezionarli consapevolmente in base alle situazioni e ai contesti. Si è concordi con Santipolo (2018a, 10) nell'affermare che la consapevolezza linguistica così definita «è pertanto anche il punto di partenza per una riflessione verso l'altro e le sue lingue o varietà linguistiche secondo i principi della Folk Linguistics (Preston 2006) e della loro applicazione in ambito di linguistica educativa (Santipolo 2012; 2016)» trasformandosi così in consapevolezza interlinguistica.

Secondo quanto sostenuto anche in Borghetti $(2018,416)$, per cui

il valore dello scambio comunicativo non risiede solo nella possibilità di interagire con la diversità, ma anche e soprattutto nel riflettere su sé stessi e sul proprio senso di appartenenza a uno o più gruppi culturali, proprio alla luce dell'incontro interculturale stesso (Byram, Zarate 1994).

se la lingua costituisce un aspetto imprescindibile dell'identità e la cultura (Kramsch 1993) è concepita in maniera intersoggettiva e dialogica e non come entità pre-esistente all'incontro (o solo in parte), la consapevolezza interlinguistica e interculturale presuppone un ulteriore passo verso l'acquisizione di quelle competenze di interazione e mediazione ormai ritenute imprescindibili per la vita in società 
complesse. Nelle società dei Paesi europei ed occidentali in generale, infatti, l'identità non è associata (né associabile) a un contesto geografico ma è piuttosto un concetto fluido e diversificato, composito, come i repertori linguistici degli individui che le compongono.

Ci si riferisce naturalmente all'impatto dei fenomeni migratori sulle società di arrivo, evidenziando che tale impatto si genera specificatamente nell'interazione tra coloro che vivono già in quelle società (da più o meno tempo) e chi si affaccia per la prima volta alla nuova realtà.

A tal proposito, nell'ambito dei processi di accoglienza e inserimento dei migranti nelle società di arrivo, un ruolo chiave è giocato dalle competenze linguistiche nella lingua target, associata alla possibilità di godere di maggiori opportunità dal punto di vista educativo-formativo, professionale, economico e sociale.

Nonostante sia ormai dimostrato in molti studi e ricerche come la valorizzazione della/e lingua/e madri e di tutte le lingue che compongono il repertorio linguistico degli studenti favorisca anche l'acquisizione della L2, molto spesso l'approccio adottato sia in contesti didattici che all'esterno si basa sulla repressione delle altre risorse del repertorio linguistico, che vengono escluse in favore della L2. Con tale approccio, però, da una parte non si valorizza il patrimonio linguistico di cui gli immigrati sono portatori, optando per una visione miope del processo di interazione con le società di accoglienza, mentre dall'altra non si permette a docenti, mediatori, operatori, e membri della comunità di aumentare la propria consapevolezza interculturale e interlinguistica, perdendo così l'occasione di sviluppare una delle competenze chiave, identificate dell'OCSE come irrinunciabili per la vita nella società contemporanea, ovvero la competenza multilinguistica, che non comprende solo la conoscenza anche parziale di più codici linguistici, ma anche le capacità di mediazione e comprensione interculturale. Queste ultime, così come la valorizzazione della diversità, sono fondamentali strumenti per la creazione di società coese, specie se gli individui riescono a svilupparle come componenti della competenza comunicativa interculturale (CCI). Questo concetto, elaborato da Balboni e Caon (2015) sulla base del modello di competenza comunicativa costruito da Balboni, prevede la capacità di agire da parte degli individui in 'eventi comunicativi interculturali', un'evenienza che ciascuno e ciascuna di noi sperimenta più e più volte al giorno. Per condurre interazioni soddisfacenti ed efficaci nell'ambito di questi eventi, gli individui devono non solo usare le proprie le abilità linguistiche ma dimostrare di essere competenti dal punto di vista interculturale, includendo quindi a pieno titolo la capacità di gestire relazioni interpersonali di questa natura. Sia quindi coloro che sono parte delle società di accoglienza da più tempo - i cosiddetti membri esperti della comunità di pratica, quindi immigrati di lungo periodo, nativi - che coloro che vi arrivano per la prima 
volta hanno la necessità di dotarsi si questo tipo di competenze, attraverso esperienze concrete di contatto con la diversità culturale. Non ci si sofferma qui sul concetto di cultura, ampiamente trattato da più autori, ${ }^{1}$ evidenziando soltanto, in questa sede, che il concetto di cultura non viene inteso in rapporto ad appartenenze geografiche né associato all'idea di esclusività, bensì collegato all'idea più ampia di interculturalità, cioè, nella definizione di Santerini (2015) a «quell'insieme di processi (psichici, relazionali, istituzionali) riguardanti gli scambi e la reciprocità tra culture, intese come totalità complesse, in rapporto dinamico tra loro». Per poter raggiungere questo obiettivo, occorre che la competenza comunicativa interculturale si sviluppi negli individui:

a. seguendo le tappe che sono state identificate nel tempo da diversi studiosi, mettendo in primis in discussione il modello culturale in cui ci si identifica - ovvero l'idea che la nostra visione del mondo sia solo una rappresentazione mentale specifica che dipende dal nostro contesto si riferimento e cercare di adottare la visione che gli altri hanno di noi (Trotta 2021);

b. mettendo in evidenza i meccanismi alla base delle pratiche discorsive, che possono trasformarsi in eventi interculturali in base a determinate condizioni che verranno descritte più avanti.

I concetti ai quali si fa riferimento per tracciare le linee teoriche dalle quali discendono le riflessioni contenute in questo capitolo, a partire dalle quali si esemplificheranno poi buone pratiche esistenti nelle società europee, sono innanzitutto il concetto di funds of knowledge (Moll et al. in Park, Valdez 2018), collegato al concetto di plurilingual resourcefulness, e i concetti di plurilinguismo e di translanguaging, proposti sia in termini di approcci teorici sia come pratiche didattiche.

Il termine e l'approccio funds of knowledge (González et al. 2005) si basano su un concetto semplice ma fondamentale: le persone sono competenti e hanno delle conoscenze, e le loro esperienze di vita hanno fornito loro queste conoscenze. A partire da questa considerazione, l'approccio promuove una visione non deficitaria degli individui che invece vengono generalmente stigmatizzati per l'appartenenza a una determinata categoria e una valorizzazione delle risorse di cui questi individui sono portatori. Molto significativo è l'esempio relativo all'analisi delle relazioni tra le famiglie immigrate di origine latina e gli educatori in una ricerca condotta negli Stati Uniti. Nell'ambito di questo approccio, gli educatori prediligono una cono-

1 Si rimanda a Bennet 2015; Hofstede 2004; Bettoni 2006 nello specifico per il rapporto lingua/identità. 
scenza diretta degli individui che sono coinvolti nei processi educativi, si avvicinano ad essi dismettendo giudizi e valutazioni preesistenti e ridanno forma alle relazioni scuola-famiglia in un'ottica completamente ribaltata. Un'altra ricerca condotta attraverso l'approccio dei funds of knowledge riguarda il contesto dell'Uganda (Kendrik, Kakuru 2012), in cui i ricercatori hanno documentato l'uso del translanguaging (ugandese-inglese) come risorsa, per bambini rimasti orfani, per costruire le relazioni con l'istituzione scolastica e conseguentemente avere una speranza per ricostruire il proprio futuro.

Proprio il translanguaging rappresenta l'anello di congiunzione tra l'approccio dei funds of knowledge e la dimensione linguistica: le risorse presenti nel repertorio linguistico di ciascun individuo costituiscono elementi chiave attraverso i quali gli individui possono gestire le molteplici relazioni discorsive selezionando e intrecciando consapevolmente le risorse che hanno a disposizione. Attraverso il translanguaging ci si propone quindi di «enabling migrants to recognize their full language repertoire and helping them to incorporate new features into their own language system» (García 2017, 17-18).

Viene quindi promossa l'idea che sia necessario, in prima battuta, riconoscere e valorizzare il plurilinguismo di cui sono portatori gli immigrati e in particolare i rifugiati, superando la visione deficitaria che stigmatizza questi individui e abbracciando invece una prospettiva legata al concetto di resourcefulness (Choi, Najar 2017), che verranno ripresi più avanti ma che nella sostanza fa riferimento all'insieme di risorse accumulate da una comunità nel tempo e impiegate per un coinvolgimento attivo e trasformativo nella società.

Una forte spinta in questo senso arriva da tempo dai documenti europei, in primis il Quadro Comune Europeo di riferimento per le Lingue, che definisce il plurilinguismo come «capacità che una persona ha di usare le lingue per comunicare e di prendere parte a interazioni interculturali, in quanto padroneggia, a diversi livelli, competenze in più lingue ed esperienze in più culture. [...] si tratta di una competenza complessa o addirittura composita su cui il parlante può basarsi» (QCER 2002, 205), un insieme di risorse quindi, strettamente interrelate le une alle altre, come peraltro sostenuto dall'ampia accettazione e diffusione della teoria dell'interdipendenza (Cummins 2001; 2005), secondo la quale le risorse linguistiche degli individui, e di conseguenza l'accesso ad ognuna delle lingue conosciute, sarebbero interconnesse nella comune facoltà di linguaggio propria degli esseri umani e nella realizzazione della competenza comunicativa.

Nel documento europeo Una sfida salutare. Come la molteplicità delle lingue potrebbe rafforzare l'Europa è chiara l'indicazione ai policymaker e governanti: il plurilinguismo è un elemento di valore dell'identità comunitaria e come tale dovrebbe essere valorizzato. A tal proposito, come sostiene Tullio De Mauro: 
La pluralità linguistica non ci sopravviene dall'esterno, come la diversità degli abbigliamenti. Ma nasce dall'interno di ciascuno di noi esseri umani. Sì, rispettare la diversità linguistica è davvero rispettare un diritto umano. Quella diversità non ha a che fare solo con la libera scelta di costumi e usanze. Essa è corradicale alla capacità di produrre e controllare variazioni e differenziazioni, alla capacità, in definitiva. Di storia cui la specie umana deve la sua origine, la sua natura più profonda, le sue possibilità di degna sopravvivenza. $(2018,117)$

La pluralità linguistica oggi si identifica quindi non solo con la presenza di dialetti, varietà o lingue di minoranze storiche, ma nella quasi totalità dei contesti europei, così come in Italia, si aggiungono ormai «cospicue comunità linguistiche minoritarie di nuovo insediamento, con intarsi e mosaici linguistici di straordinaria varietà e vitalità» (De Mauro 2018, 105).

L'estesa presenza, in Europa in particolare, di lingue di nuovo insediamento portate dall'immigrazione, ci pone dinanzi all'esigenza di riflettere sul significato concreto dell'espressione 'consapevolezza interlinguistica e interculturale' e su come essa può realizzarsi nei nostri spazi, fisici e metaforici, di vita quotidiana. È opportuno ampliare la prospettiva dalla quale analizzare il fenomeno prendendo in considerazione una visione transdisciplinare: dalla geografia ci arrivano infatti alcune suggestioni che possono aiutare a rappresentare concretamente quanto descritto fino a ora.

Le esperienze migratorie hanno infatti avviato una trasformazione antropologica degli spazi e delle strutture in cui si svolge quotidianamente la vita: le città rappresentano il luogo privilegiato in cui è possibile osservare dinamiche di cambiamento indotte da molteplici fattori, fra i quali i fenomeni migratori.

Non è semplice dipanare la matassa di spinte economiche, sociali, tecnologiche, o migratorie cui la città è soggetta nonché osservare attraverso la giusta lente le dinamiche conservative o di cambiamento che nelle città avvengono (Cattedra 2014): quel che è certo, però, è che gli spazi pubblici «intesi come motori della rigenerazione e dell'animazione di vecchi e nuovi spazi delle città», sono i luoghi dove meglio si esprimono le dinamiche cui si è fatto cenno in precedenza, dei formidabili 'dispositivi di socializzazione' (Joseph 1991 in Cattedra 2014, 252). Costituiscono infatti «luoghi di passaggio, di incontro, di passeggio e di passaggio di pratiche collettive più o meno spontanee o organizzate», essendo le città sempre più 'interetniche':

la globalizzazione ha reso più evidente la crisi dell'idea dello Stato che coincide con un'unica lingua, cultura e religione, come del resto ha posto in discussione l'idea tradizionale di luogo come chiuso e stabile (Massey, Jess 1992). Il luogo come entità unicamente 
fisica e delimitata conduce a una compattezza etnica che non è mai esistita; i luoghi, anche i più isolati, sono sempre stati aperti agli scambi. Ed è in questi incontri che ciascuno di noi trova delle reti di relazioni che formano un altro modo di considerare il concetto di luogo e lo stesso concetto di identità. (De Spuches 2014, 164)

I contesti urbani, nello specifico, costituiscono il luogo in cui questa vitalità si manifesta, rendendo palese il collegamento tra le forme linguistiche in uso e l'identità; ${ }^{2}$ sono i contesti in cui emerge la trama delle relazioni dei migranti con le comunità di appartenenza e con quelle di arrivo, in cui si realizzano le rappresentazioni della diaspora.

L'approccio del Linguistic Landscape (d'ora in avanti LL) è un'area di studio che ha saputo mettere insieme diverse discipline - linguistica, sociolinguistica, antropologia, geografia - nell'idea che il panorama linguistico rappresenti «the scene where the public space is symbolically constructed» (Shohamy et al. 2010, xi), connotata proprio attraverso gli usi linguistici che all'interazione pubblica danno forma. Lavori più o meno recenti (a partire da Gramsci) dimostrano come le dinamiche che coinvolgono gli usi linguistici nelle città sono dinamiche che danno forma a relazioni di potere. La scelta quindi di estromettere o includere più lingue o culture nella comunicazione nell'ambito dei contesti urbani attraverso varie forme (politiche linguistiche, politiche educative, pratiche quotidiane che derivano da entrambi) è una scelta con cause e conseguenze di natura politica: si vedano ad es. nel volume curato da Shohamy, Ben Rafael, Barni (2010) lo studio condotto da De Klerk e Wiley o ancora lo studio sull'uso dell'ebraico a Jaffa e Tel Aviv, di Waksman e Shohamy.

Le pratiche plurilingui di translanguaging sono al giorno d'oggi estremamente diffuse nelle città; gli individui interagiscono spesso combinando elementi di diverse lingue e codici semiotici, soprattutto durante le interazioni sui social media e in tal modo sia i paesaggi linguistici individuali sia paesaggi linguistici pubblici (Mayr 2021, 191) sono caratterizzati dalla compresenza di più codici nell'uso dei parlanti.

I molti rifugiati adulti che popolano le classi di L2 in tutti i Paesi europei sono portatori di repertori plurilingue (D’Agostino, Sorce 2016; D'Agostino 2017), che possono essere riconosciuti e valorizzati in maggiore o minor misura a seconda delle politiche linguistiche formali o delle pratiche didattiche informali. Provengono infatti da società in cui le persone comunicano abitualmente in due o più lingue: la lingua parlata in famiglia può non coincidere con la lingua

2 Per un approfondimento su questo concetto si vedano Vedovelli 2010; Shohamy et al. 2010 . 
di scolarizzazione; la lingua di comunicazione con vicini o amici può non coincidere con la lingua parlata in famiglia o insegnata a scuola.

Per valorizzare tale ricchezza in ambito didattico, sarebbe fondamentale fare tesoro di alcune delle indicazioni contenute in documenti redatti nel tempo da coloro che si sono occupati di integrazione linguistica dei migranti adulti. Uno degli strumenti più validi in tal senso è il Toolkit di supporto linguistico per rifugiati adulti, che invita tutti coloro che sono protagonisti di azioni di supporto linguistico a:

- tenere conto del fatto che il bagaglio di esperienze di cui gli immigrati sono portatori è diversificato, così come i livelli di istruzione e di alfabetizzazione, la condizione sociale, la familiarità con le lingue;

- consentire ai rifugiati con lo stesso background linguistico di aiutarsi a vicenda nella loro lingua comune o di aiutarsi usando lingue veicolari in comune (come le lingue di colonizzazione);

- mostrare interesse per le lingue di origine, cercando di stabilire delle connessioni con le L2 target;

- considerare il fatto che all'interno dei repertori linguistici possono essere presenti delle lingue apprese nei Paesi di transito, nei lunghi percorsi verso l'Europa (cf. Benucci 2017; Benucci, Grosso 2015; 2017);

- tracciare il profilo linguistico degli apprendenti per riconoscere il loro patrimonio e consentire sia a docenti/mediatori che studenti una maggior consapevolezza interlinguistica e interculturale;

- tener conto del fatto che alcuni apprendenti provengono da contesti in cui non viene usato il codice scritto per la comunicazione (vedi cap. 5 di Grosso in questo volume);

- riconoscere nell'età un valore per la consapevolezza interlinguistica: i migranti anziani possono avere competenze in più lingue ed essere predisposti al loro uso in contesti e con interlocutori diversi.

A tal proposito, è opportuno aggiungere che un recente report di ricerca, Putting Language on the Map in the European Refugee Response, stilato dall'associazione Translators Without Borders nell'ambito dell'iniziativa Mixed Migration Platform ${ }^{3}$ ha messo in evidenza, attraverso interviste qualitative condotte con operatori e migranti,

3 Il report (2017) è stato prodotto da Translators Without Borders nell'ambito della Mixed Migration Platform (https://translatorswithoutborders.org/wp-content/ uploads/2017/04/Putting-language-on-the-map.pdf). La Mixed Migration Platform (MMP) è un'iniziativa congiunta di ONG che fornisce informazioni di qualità sulla migrazione 'mista' per la politica, la programmazione e il lavoro di advocacy, nonché informazioni cruciali per le persone in movimento. La piattaforma è stata creata da sette partner - ACAPS, Danish Refugee Council (DRC), Ground Truth Solutions, Internews, INTERSOS, REACH e Translators Without Borders (TWB) - e funge da hub informati- 
che il mancato riconoscimento delle lingue potenzialmente presenti nel repertorio linguistico dei rifugiati accolti nei punti di sbarco può essere causa di disorientamento e fraintendimento. L'uso del criterio del Paese di origine per l'attribuzione di una lingua per la quale offrire il supporto di un mediatore/interprete si è rivelato ampiamente insufficiente, dal momento che in molti Paesi la lingua di comunicazione si diversifica dalla lingua ufficiale a seconda del distretto territoriale, sebbene i rifugiati abbiano spesso una competenza di ricezione della lingua ufficiale. Si veda ad es. il caso dei rifugiati iracheni, le cui lingue di origine possono non coincidere con l'arabo, includendo il sorani (varietà del curdo), il turkmeno o la lingua zaza. La tabella 1 indica chiaramente la discrepanza tra le lingue presenti nei servizi di accoglienza e le lingue dei migranti, definite in maniera molto precisa 'lingue meno comunemente supportate'.

Tabella 1 Lingue comunemente e meno comunemente supportate negli interventi di mediazione linguistico-culturale in Italia, Grecia e Turchia

\begin{tabular}{lll}
\hline Country & $\begin{array}{l}\text { Main languages } \\
\text { of communication }\end{array}$ & $\begin{array}{l}\text { Less commonly supported } \\
\text { languages }\end{array}$ \\
\hline Greece & Arabic, English, Greek, & $\begin{array}{l}\text { Bengali, French, Lingala, Kurmanji, } \\
\text { Pashto, Sorani, Urdu, Uzbek }\end{array}$ \\
\hline Persian & English, French, Italian & Amharic, Arabic, Bambara, \\
& Bengali, Berber, Fulani, Kurmanji, \\
& Ika, Mandinka, Oromo, Pashto, \\
& Somali, Tigre, Tigrinya, Temne, \\
& Urdu, Wolof, Zawa \\
\hline Turkey & Arabic, English, Turkish & Kurmanji, Pashto, Persian Somali, \\
& & Sorani \\
\hline
\end{tabular}

Fonte: report Putting language on the map $(2017,8)$

Le interviste a rifugiati e operatori presenti nel report citato raccontano una realtà caratterizzata da parte dei rifugiati dalla percezione che la comunicazione, nei punti di accoglienza, non sia stata loro trasmessa nella lingua in cui possono comprendere meglio informazioni fondamentali, come ad es. quelle fornite in un presidio sanitario, mentre da parte degli operatori dell'accoglienza la scelta della lingua maggiormente diffusa o la comunicazione a gesti viene ritenuta sufficiente. Un altro aspetto evidenziato dal report riguarda l'uso di lingue ponte coloniali, come l'inglese e il francese, la cui conoscenza risulta molto ridotta soprattutto in parlanti che non hanno avuto 
accesso a percorsi di scolarizzazione strutturati o li hanno interrotti dopo pochi anni. In questo caso, la limitata conoscenza di queste lingue da parte dei rifugiati si associa alla limitata conoscenza anche da parte degli operatori, per la maggior parte dei quali è una lingua straniera, determinando una comunicazione poco efficace.

Risulta chiaro come tali scelte siano dettate dall'insufficienza di risorse dedicate ai servizi di language support, mentre sarebbe opportuno, come suggeriscono i redattori del report, orientare la ricerca nella direzione dell'identificazione ampia ed esaustiva dei repertori linguistici dei migranti.

Accrescere la consapevolezza interlinguistica e interculturale in questo senso significa quindi:

1. far circolare maggiormente informazioni attendibili sulle risorse linguistiche di cui sono in possesso i migranti e usarle per facilitarne l'integrazione in contesti di accoglienza, didattici e quotidiani;

2. dare avvio a processi di meta riflessione a partire da informazioni e concetti condivisi.

Fra le preziose iniziative del Consiglio d'Europa, si annovera la creazione di una pagina web, Integrazione Linguistica dei Migranti Adulti (ILMA), ${ }^{4}$ in cui è visibile una sezione che contiene informazioni snelle e accessibili su vari concetti chiave: alfabetizzazione/analfabetismo; competenza/competenza plurilingue; prima lingua; apprendimento informale; bisogni linguistici; gruppi vulnerabili.

La consultazione di queste voci può fornire ai docenti e a tutti i cittadini che intendano ampliare il bagaglio di conoscenze per partecipare attivamente al processo di inclusione sociale dei migranti adulti una prima base molto utile per poi poter espandere e approfondire il processo di consapevolizzazione che coinvolge tanto gli immigrati quanto coloro che vivono da più tempo (o da sempre) nelle società di arrivo, per permetterne un'interazione sempre più significativa e che sia indirizzata all'accrescimento dell'inclusione e della coesione sociale.

Come sostiene De Mauro, infatti, commentando una sua relazione tenuta al Congresso Internazionale dei linguisti, nel 1973:

lo sviluppo delle società moderne [...] funziona in modo tale che sradica molti dalle loro matrici linguistiche, ma non gli consente l'acquisizione di altre matrici, radici, nicchie linguistiche. Disimparano il tupi, l'haussa o il calabrese, ma non imparano il portoghese, il francese o l'italiano. Nascono così degli sradicati linguistici. (De Mauro 2018, 57)

4 https://www.coe.int/it/web/lang-migrants/forms-of-linguistic-integration. 
Accrescere la consapevolezza interculturale e interlinguistica significa quindi innanzitutto osservare le interazioni tra individui in tre dimensioni centrali, ovvero il punto di vista affettivo (che riguarda i bisogni e le necessità di ogni singolo individuo), il punto di vista cognitivo (ovvero il modo in cui i membri di una determinata cultura percepiscono la realtà, secondo alcune categorie come spazio/tempo, mascolinità/femminilità, stereotipi) e il punto di vista comunicativo-comportamentale, ovvero la reazione degli individui ai contributi di altre culture. Molti studiosi (ad es. il già citato Bennet 2015) hanno descritto lo sviluppo di competenze comunicative interculturali come un processo lineare, che presuppone un passaggio dalla fase etnocentrica, in cui l'individuo riesce a considerare il mondo esclusivamente da una prospettiva monoculturale e monolingue, a una fase etnorelativa, in cui riesce invece a vivere la propria cultura mettendola in relazione agli altri. Fra le due fasi sono descritti naturalmente numerosi passaggi intermedi, che presuppongono un'acquisizione graduale della consapevolezza interculturale.

Risulta forse però opportuno ripensare tale processo come un processo simile a quello di acquisizione linguistica, in cui i parlanti (sia il membro esperto della comunità di pratica che il novizio, per usare termini cari alla visione della comunità di arrivo come comunità di pratica):

1. compiono dei tentativi per verificare le proprie ipotesi sul funzionamento della cultura/delle culture con cui si trovano a interagire;

2. costruiscono conoscenza interculturale nell'ambito dell'interazione stessa con i membri esperti.

È necessario, come afferma Borghetti $(2018,414)$, definire «come conoscenze, atteggiamenti e abilità interculturali in via di sviluppo possono essere manifestate - e quindi studiate - a livello di performance comunicativa». Per fare ciò, è importante analizzare attraverso lenti che permettono di osservare a livello micro le interazioni per individuare come i fenomeni interculturali si manifestano in pratiche linguistico - discorsive, per esempio attraverso l'analisi della conversazione (Pallotti 2007).

Per le ragioni esposte sopra, il punto di contatto tra la consapevolezza interculturale e interlinguistica è facilmente identificabile se si assume come definizione di 'parlante interculturalmente competente' quella contenuta in Borghetti $(2016,424)$ : «colui che, cogliendo in tempo reale le affiliazioni culturali degli interlocutori attraverso i loro usi linguistici, sa avvalersi del proprio repertorio plurilingue per negoziare le loro identità così come le proprie». Questo concetto trova il suo riflesso diretto, dal punto di vista delle competenze interculturali, nel sapersi dotare di un'identità che non costringe ad aderire esclusivamente a un modello ma che piuttosto permette di 
selezionare elementi dalle diverse culture alle quali si appartiene e condividerli in un'interazione.

Nella parte successiva dell'articolo si cercherà, sulla scorta di quanto suggerito in Borghetti (2018), di compiere un passo ulteriore per la ricerca su questi temi:

- analizzando incontri 'potenzialmente interculturali' attraverso l'AC per cercare di comprendere quali strumenti linguistici sono stati utilizzati dai parlanti;

- descrivendo esempi di buone pratiche finalizzate all'acquisizione della consapevolezza interlinguistica e interculturale, in particolare in contesto formativo e didattico.

\subsection{Osservare le lingue e le culture}

Lo sviluppo della consapevolezza interlinguistica e interculturale è molto spesso inconscio, o per meglio dire, i parlanti apprendono, come si diceva in precedenza, dai molteplici tentativi e nel confronto con altri parlanti. Se i tentativi offrono conferme positive, rafforzano le ipotesi verificate dai parlanti stessi. Tutti abbiamo in mente (e d'altra parte è noto in letteratura) l'utilizzo strumentale dei dialetti e delle varietà locali in Italia da parte dei migranti, con il fine di 'mimetizzarsi' linguisticamente il più possibile con i parlanti nativi o autoinvestirsi di tratti identitari della C2 o ancora di mettere in atto forme di captatio benevolentiae finalizzate a concludere positivamente una transazione o un incontro (ad es. vendere un oggetto, instaurare una relazione amicale/di contatto).

Questo avviene tanto in contesti informali, e nelle interazioni quotidiane, quanto in contesti didattici, in cui il fenomeno può diventare oggetto di riflessione interlinguistica e interculturale per tutta la classe.

Gli esempi proposti, tratti da contesti didattici e non, sono caratterizzati dall'uso consapevole delle lingue presenti nei repertori linguistici e corrispondono a quelle che, nel già citato studio di Borghetti, sono definite come «sequenze che sembrano più promettenti di altre per l'apprendimento linguistico interculturale» $(2018,421)$, ovvero che mettono in evidenza da parte degli apprendenti una riflessione (implicita o esplicita) di tipo metalinguistico e metaculturale.

L'esempio (1) è tratto dallo studio condotto da Grosso e Floris (2020) su interazioni un contesto didattico specifico: l'ambito sanitario. ${ }^{5}$ Alla battuta 75, l'insegnante fa uso della lingua madre degli studenti (dichiarando i propri limiti riguardo alla competenza lingui-

5 Il frammento selezionato fa parte del corpus di interazioni raccolte da Mohana Floris in occasione della ricerca-azione condotta nell'ambito di un corso di italiano per stranieri indirizzato ad apprendenti arabofoni (iracheni) in Italia per motivi sanitari ed è stato più ampiamente contestualizzato e commentato in Grosso, Floris 2020. 
stica) per rovesciare la relazione asimmetrica docente-apprendenti attribuendo a questi ultimi maggior potere comunicativo.

(1) 75: /INS/ Tis'a 'ašra dieci GIUSTO (0.3) <in arabo ricordo solo il numero dieci> I remember just the number ten in arabic

76: /STUD2/ ((ride)) sì `ašra (0.4) Šway šway

77: /INS/ Šway šway

Ancora, nell'esempio (2) alla battuta 690, l'insegnante usa la parola buma ${ }^{6}$ per ratificare quanto affermato scherzosamente in precedenza da uno degli studenti e successivamente chiede conferma della comprensione da parte degli studenti sempre attraverso il ricorso a un'espressione in arabo, mahfum? In questo caso, oltre a usare la L1 degli studenti, l'insegnante evidenzia la propria competenza di parlante interculturale facendo uso di un'espressione metaforica, che come noto in letteratura costituisce uno degli ambiti più ostici per gli apprendenti di una L2 proprio perché fortemente collegati ad aspetti culturali (Grosso, Pitzanti 2019). Quindi, da un lato, la docente mostra una conoscenza della specifica lingua e cultura degli studenti, dall'altro evidenzia una grande consapevolezza interlinguistica e interculturale nella scelta di elementi che connotano i suoi usi linguistici come we code, codice condiviso che può permetterle di stabilire un rapporto privilegiato con gli studenti.

(2) 689: /STUD1/non è bella lui

690: /INS/ lui è un būma ((i ragazzi ridono)) capito? Mafhūm?

691: /STUD1/ capito capito

Come osservato negli esempi (1) e (2), l'appropriazione e l'uso della L1 dell'interlocutore (crossing) fanno parte di meccanismi di allineamento conversazionale tramite i quali si realizzano le strategie di convergenza e accomodamento (Grosso 2015). Il crossing consente di fatto la 'rottura' dei confini determinati dall'asimmetria essendo definito come una strategia che

involves code alternation by people who are not accepted members of the group associated with the second language that they are using (code switching into varieties that are not generally thought to belong to them). This kind of switching involves a distinct sense of movement across social or ethnic boundaries and it raises issues of legitimacy which, in one way or another, participants need to negotiate in the course of their encounter.

6 Nella varietà di arabo parlata in Iraq la parola gufo viene impiegata per definire un ragazzo/a poco attraente. 
L'aspetto rilevante è quindi l'opportunità che questa tipologia di code-switching offre ai parlanti di valicare i confini etnici o di gruppo auto-attribuendosi un'identità linguistica che crei maggiore vicinanza con l'interlocutore e mostrandone quindi la competenza come parlanti interculturali.

Il terzo esempio, invece, è tratto da una conversazione spontanea raccolta su un luogo di lavoro. I due interlocutori sono un parlante nativo (siciliano) e un non nativo (peruviano). Il turno è appena iniziato, e il lavoratore peruviano protesta con il collega per il ritardo con cui è arrivato al lavoro. L'atto della protesta, come indicato in molti studi recenti e meno recenti è uno degli atti comunicativi che mettono maggiormente a rischio la faccia dell'interlocutore (Bettoni 2006; Trubnykova 2021), «un atto comunicativo di per sé rischioso per la relazione tra gli interagenti» (Leone 2011, 103). Come dimostrato da Liddicoat (2016), Orletti (2000), le interazioni tra esperti e non esperti di appartenenti a una determinata comunità di pratica (coincidenti in questo caso con un parlante nativo e uno non-nativo) sono spesso caratterizzate da un rapporto di asimmetria, in cui gli interlocutori esperti esercitano potere nei confronti dei non esperti.

Nell'esempio (3), tuttavia, la situazione è ribaltata, nel senso che è proprio il parlante ispanofono, violando le norme socio-pragmatiche, a minacciare la faccia dell'interlocutore. La violazione delle norme, nello specifico, si manifesta in due atteggiamenti: il primo (violazione delle norme pragmalinguistiche a livello testuale) consiste nella reiterazione eccessiva dell'elemento che costituisce la prima parte della protesta, ovvero l'atto espressivo tramite il quale l'interlocutore formula il proprio disappunto nei confronti del comportamento 'scorretto' del parlante non nativo, e cioè del ritardo nell'arrivare sul posto di lavoro; il secondo (violazione delle norme sociopragmatiche) consiste nel sanzionare negativamente un comportamento verso il quale nella cultura italiana, a seconda dei contesti, esiste una certa elasticità: il ritardo (Van Berne 2016; Minascurta 2017). 


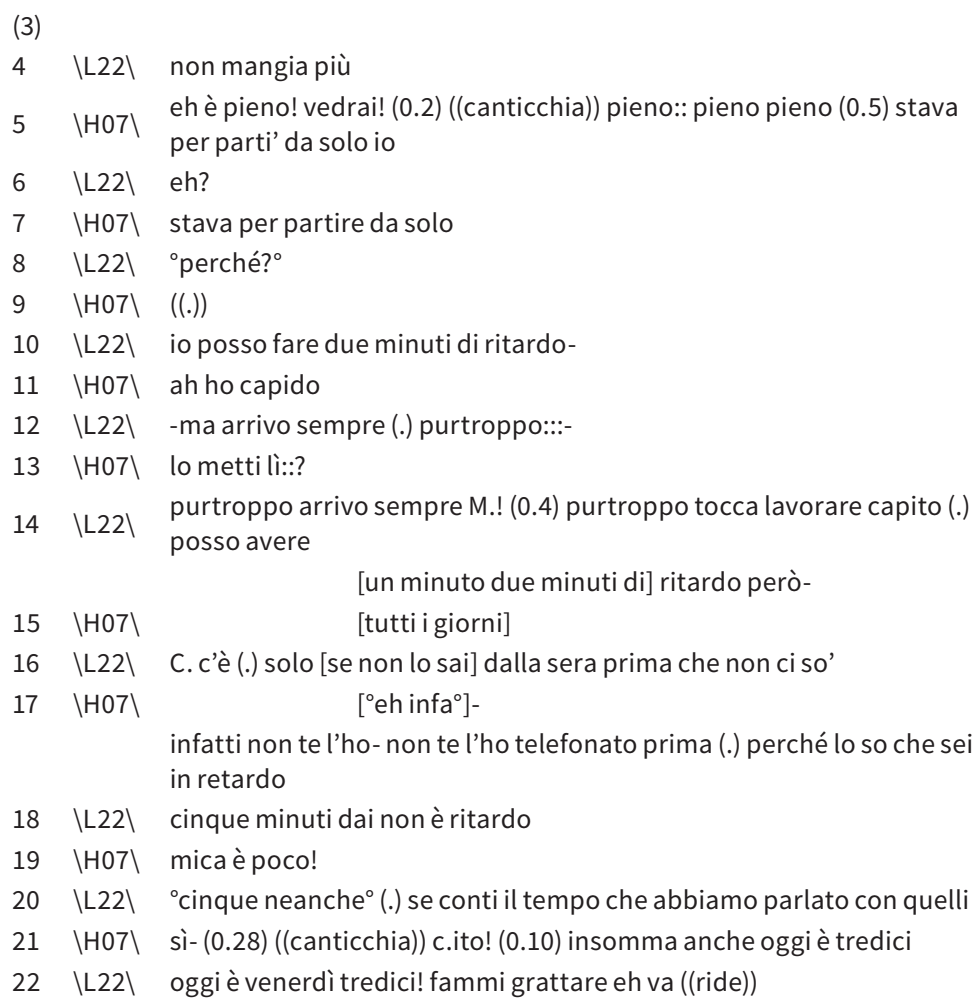

Alla battuta 5, il parlante peruviano esprime l'atto espressivo che manifesta la critica 'informando' anche sullo stato psicologico del parlante; dopo la richiesta di chiarimento del collega l'interlocutore ripete la prima parte della protesta, enfatizzando attraverso la voce l'espressione da solo; dopo l'ulteriore richiesta di chiarimento del parlante nativo (perché? battuta 9), segue il silenzio del collega peruviano, a sottolineare la gravità del comportamento, e successivamente la giustificazione dell'interlocutore italofono: io posso fare due minuti di ritardo. La giustificazione viene interrotta da un commento ironico (ah, ho capido) del collega peruviano e ripresa alla battuta successiva (ma arrivo sempre (.) purtroppo!) in cui il parlante italofono sminuisce la propria agentività attraverso l'uso del verbo alla forma impersonale (Purtroppo tocca lavorare!) e del mitigatore 'purtroppo'. Anziché assumere un atteggiamento di accomodamento convergente, però, il parlante non nativo insiste nell'attribuire al parlante italofono un'abitudine negativa, quella di arrivare tutti i giorni (battuta 15) in ritardo, minacciandone la faccia.

All'ennesima autoassoluzione del parlante nativo, l'interlocutore risponde commentando che in effetti non ha sollecitato l'arrivo del 
collega solo perché conosce a fondo la sua scarsa puntualità (no te ho chiamato perché lo so che sei in retardo). Da quest'ultima affermazione desumiamo che la protesta scaturisce non tanto da una situazione contingente di difficoltà causata dal ritardo del lavoratore, quanto dall'insofferenza per una situazione che pare essersi cronicizzata.

Infine, dalla battuta 18 in poi, pare finalmente emergere il nucleo del fraintendimento alla base della discussione tra i due colleghi: mentre per il parlante italofono cinque minuti non è ritardo, lo stesso lasso di tempo è percepito come piuttosto lungo dal collega peruviano, che per rimarcare il concetto fa uso del rafforzativo ( $\mathrm{mi}$ ca è poco! battuta 19). Dopo l'ultimo tentativo da parte del parlante italofono di giustificarsi, attraverso un'attenuazione (cinque minuti neanche!) il dialogo si conclude con un repentino cambio di argomento da parte del parlante peruviano (topic change), che sancisce la chiusura della sequenza di protesta (e di difesa da parte dell'interlocutore), e l'inizio di un atteggiamento di convergenza e di accomodamento nei confronti dell'interlocutore italofono, cui il parlante peruviano si rivolge abbreviandone il nome con un suffisso diminuitivo spagnolo, -ito.

Sensibilizzare quindi i cittadini, immigrati e non, agli aspetti relativi alle componenti pragmatica e socio-culturale della competenza comunicativa permette di migliorare le loro interazioni, consentendo loro di raggiungere lo status di parlanti interculturalmente competenti, e conseguentemente di favorire la coesione sociale delle società di arrivo. Al contrario, si rileva come nella comunicazione quotidiana che avviene in contesti in cui non si è intrapreso un percorso guidato in tal senso possano verificarsi con maggiore frequenza episodi di fraintendimenti, che possono arrivare a minacciare la coesione sociale nella comunità di accoglienza.

Per quanto riguarda gli apprendenti di L2, negli ultimi anni lo studio e l'insegnamento della componente pragmatica della competenza comunicativa hanno assunto un ruolo fondamentale nella linguistica applicata e nella didattica dell'italiano come L2. Come è noto in letteratura (cf. Nuzzo, Gauci 2012; Grosso 2015), se nei confronti dell'errore grammaticale o sintattico i parlanti nativi mostrano una maggiore tolleranza è verso l'errore pragmatico che si dimostrano più inflessibili sanzionando negativamente il parlante non nativo.

Il filone di studi legato all'intercultural pragmatics ha indagato, fra gli altri, il fenomeno del transfer pragmatico, concentrandosi sul cosiddetto fraintendimento interculturale, per analizzarne le caratteristiche che riguardano l'aspetto pragmalinguistico e sociopragmatico. Numerosi studi, come quelli riportati in Nuzzo, Gauci (2012), Kasper, Blum-Kulka (1993), Kasper, Rose (2003), descrivono processi in cui il trasferimento di norme sociopragmatiche dalla prima alla seconda lingua induce il parlante ad agire in accordo con le norme sociali tipiche della lingua/cultura di provenienza, che possono ri- 
sultare in conflitto - o quanto meno non coincidenti - con quelle della lingua/cultura di arrivo.

L'acquisizione della competenza socio-pragmatica, così come di quella pragmalinguistica, risulta infatti fondamentale per l'integrazione positiva dei migranti nelle società di accoglienza, proprio perché queste due componenti della competenza comunicativa rappresentano l'anello di congiunzione, o per meglio dire, il punto di contatto tra competenza linguistica e interazione sociale. Secondo Palermo $(2013,31)$ «anche nella comunicazione quotidiana la mutua volontà di conferire e attribuire senso concedono enormi spazi di manovra per comunicare in situazioni 'difficili', superando lacune vistose nella coesione grammaticale»; tuttavia, in alcuni casi tale atteggiamento cooperativo da parte dei parlanti, già definito di convergenza comunicativa (Grosso 2015), appare sgretolarsi. In questo caso, gli errori pragmatici vengono attribuiti alla personalità del parlante o a generiche caratteristiche della nazionalità/della cultura di appartenenza, generando una spirale di pregiudizio difficilmente decostruibile specie se non c'è una corrispondenza tra forma linguistica e intenzioni comunicative, poi, genera forme reciproche di razzismo linguistico.

Alla luce di quanto affermato fino a ora, è importante chiedersi: come possiamo sviluppare la consapevolezza interlinguistica e interculturale attraverso attività didattiche?

Per avviare azioni di sensibilizzazione in tal senso esistono numerosi strumenti a disposizione sia dei membri che già fanno parte delle comunità di arrivo che dei neoarrivati. Si presenteranno qui alcuni di essi con lo scopo di individuare quelli che possono essere considerati buone pratiche per le loro caratteristiche.

\section{L'Autobiografia degli Incontri Interculturali}

Nel 2009 il Consiglio d'Europa pubblica l'Autobiografia degli Incontri Interculturali, ${ }^{7}$ elaborata per la Divisione delle Politiche Linguistiche del Consiglio d'Europa da Michael Byram, Martyn Barrett, Julia Ipgrave, Robert Jackson, María del Carmen Méndez García, con l’obiettivo di predisporre uno strumento che, attraverso le riflessioni indotte da una specifica esperienza interculturale avuta nella vita, permetta agli individui di collegare questa esperienza a sentimenti ed emozioni e, cosa ancor più importante, a 'indossare i panni degli altri'. Ecco così che questo strumento, di facilissima compilazione, getta i ponti per un dialogo basato sulla personalizzazione dell'espe-

7 Il documento è disponibile alla pagina: https://rm.coe.int/autobiografia-degli-incontri-interculturali/16806bfe2c. 
rienza del contatto interculturale, induce a riflettere tra similarità e differenze tra gli individui, con domande che elicitano specifiche esperienze legate alla lingua e alla comunicazione, sia linguistica che extralinguistica.

\section{Supporto linguistico per rifugiati adulti:}

il Toolkit del Consiglio d'Europa

Nell'ambito di questa pubblicazione, sperimentata nel 2017 e finalizzata a fornire una 'cassetta per gli attrezzi' volta a facilitare il percorso di integrazione linguistica, con l'intento di promuovere l'inclusione sociale, la coesione sociale e il rispetto per la diversità, si segnala come fondamentale per accrescere la consapevolezza interlinguistica e interculturale, l'uso degli strumenti 27 («I profili linguistici dei rifugiati»), 28 («Scoprire le risorse linguistiche e capacità dei rifugiati») e 29 («Le cose più importanti da apprendere secondo i rifugiati»), 30 («Osservare le situazioni in cui i rifugiati hanno bisogno di usare la lingua del Paese ospitante») e 38 («Il ritratto plurilingue»). Quello che risulta particolarmente interessante e utile di questi strumenti è la versatilità nel rivolgersi sia a coloro che, da membri esperti della comunità di arrivo, offriranno supporto agli adulti rifugiati, sia ai rifugiati stessi. Gli esercizi proposti prevedono, così come necessario per ottenere un aumento autentico della consapevolezza interlinguistica e interculturale, l'elaborazione di riflessioni sulla/e propria/e lingue e culture e soprattutto gli effetti delle relazioni fra esse. Fra le attività proposte, molto utile può risultare cimentarsi in esercizi che prevedono un accostamento a testi in lingue in alfabeti diversi dal proprio, per sperimentare la sensazione di straniamento che potrebbero provare gli studenti rifugiati bassamente scolarizzati/analfabeti/provenienti da alfabeti non latini quando si immergono nelle società occidentali, altamente testualizzate.

\section{Buone pratiche ed esperienze nei contesti formativi}

Sebbene in molti contesti prevalga ancora un approccio monolingue e di repressione delle lingue di origine (Malandrino 2021), in molti Paesi europei e non, si annoverano diverse esperienze didattiche finalizzate all'accrescimento della consapevolezza interlinguistica e interculturale che sono state condotte in contesti formativi, nelle scuole e nell'ambito dei centri di formazione per gli adulti, di cui si proporrà qui una breve panoramica, citando alcuni esempi.

Cominciando dai Paesi scandinavi, sia la Svezia che la Finlandia hanno sistemi educativi che promuovono l'equità e la parità. Tuttavia, i recenti cambiamenti sociali e politici legati all'aumento dell'im- 
migrazione hanno creato nuove sfide negli sforzi per sostenere la diversità linguistica. Nei curricoli nazionali della scuola dell'obbligo il multilinguismo è rappresentato utilizzando il quadro di orientamento linguistico: la lingua come problema, diritto o risorsa, evidenziando delle differenze tra i due Paesi. Mentre in Finlandia esiste un discorso esplicito sull'educazione multilingue, con l'obiettivo di integrare le prospettive multilingue nell'intero curriculum, in Svezia, invece, il discorso è meno esplicito, e il multilinguismo come concetto è limitato agli studenti di lingua minoritaria (Paulsrud 2020).

Nell'ambito della ricerca sulle modalità attraverso le quali le politiche linguistiche recepiscono il plurilinguismo presente nelle società occidentali, un recente lavoro (Smythe 2020) evidenzia analogie e differenze tra contesti educativi in Francia e in Nuova Zelanda. In entrambi i Paesi il principio al quale si ispirano le politiche e le pratiche è quello della language inclusiveness; tuttavia, mentre in Francia il plurilinguismo viene promosso dall'alto, con indicazioni nazionali che vengono recepite scarsamente dal basso e cioè nei contesti scolastici e formativi, e con un'attenzione prevalente alle lingue più rappresentante in termini di numero di parlanti come l'arabo, in Nuova Zelanda, invece, il discorso politico su questo tema è meno esplicito. In questo Paese, tuttavia, vengono realizzate un maggior numero di azioni concrete indirizzate a gruppi specifici di studenti (per lo più appartenenti alle minoranze Maori, Pasifika), con il risultato che almeno 17 lingue vengono insegnate nelle scuole neozelandesi. Secondo lo studio di Erdocia (2020), in Catalogna, benché il documento re-

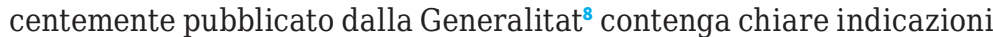
sulla necessità di pratiche didattiche plurilingui nei contesti educativi, la realtà riflette un approccio tendenzialmente monolingue, centrato sul catalano come lingua veicolare, e in cui l'approccio del translanguaging pare essere solo ostentato e non praticato in classe.

In ambito italiano molte sono state le ricerche con interessanti ricadute applicative condotte in questa direzione: fra le più recenti e significative è opportuno citare la ricerca-azione di Andorno, Sordella Con parole mie (2021), focalizzata sul coinvolgimento delle famiglie e delle lingue di origine degli studenti per il rafforzamento delle competenze CALP e dell'educazione plurilingue; o ancora l'utilizzo della pratica del translanguaging in classe sia con studenti adulti richiedenti asilo (Angelini 2021), sia con bambini e ragazzi per l'aumento delle competenze di language awareness (Mayr 2021; Carbonara, Scibetta 2018). In particolare, il progetto realizzato da Carbonara e Scibetta, L'Altroparlante, ha promosso pratiche didattiche (ad es. attraverso la scrittura o la realiz-

8 Generalitat de Catalunya (2018). El model lingüístic del sistema educatiu de Catalunya - L'aprenentatge i l'ús de les llengües en un context educatiu. Departament d'Ensenyament: http://ensenyament.gencat.cat/web/.content/home/departament/publicacions/monografies/model-linguistic/model-linguistic-Catalunya-CAT.pdf. 
zazione di ritratti plurilingue) legate alla valorizzazione dei repertori plurilingui dei ragazzi, partendo dalla ricognizione del LL della classe, e di formazione del corpo docenti in scuole di diverse regioni d'Italia. Gianollo, Fiorentini e Grandi (2020) hanno invece realizzato un'interessante ricognizione relativa alla presenza e alla percezione delle lingue immigrate nelle scuole di Bologna, e fornito una descrizione delle principali lingue immigrate presenti nei contesti scolastici italiani, analizzandole in prospettiva contrastiva rispetto all'italiano e come spunti per attività didattiche inclusive. Una ricognizione sulle molteplici lingue e sulle culture presenti nel contesto carcerario, insieme ad azioni di consapevolizzazione e formazione degli operatori penitenziari, è stata realizzata attraverso i progetti DEPORT (in ambito italiano, Benucci, Grosso 2015) e RiUscire (in ambito europeo, Benucci, Grosso 2017). Infine, si ricorda la proposta di utilizzo dell'intercomprensione come pratica didattica di translanguaging con pubblici svantaggiati avanzata da Benucci $(2021,35)$, che include l'uso della lezione capovolta come esempio di attività da proporre, dal momento che

La 'lezione capovolta' per una classe in contesto 'svantaggiato' richiede infatti una forte riflessione metalinguistica e metaculturale proponendo agli apprendenti di rispondere (utilizzando la L2, la L1 o altre lingue ponte e altri codici anche non linguistici) a una serie di domande iniziando a esporre i propri punti di vista agli altri e al docente/apprendente (ipotetico) o a raccontarsi.

\section{L’Autobiografia linguistica}

Proprio per stimolare ed elicitare il racconto di sé, un altro strumento molto valido per rafforzare la consapevolezza interlinguistica e interculturale, molto usato anche nelle classi con persone a bassa/nulla scolarizzazione (vedi cap. 5), è la pratica dell'autobiografia linguistica. Molto diffusa in antropologia, e in particolare nell'ambito di approcci etnografici, l'autobiografia linguistica è un percorso di narrazione tramite il quale l'individuo ricostruisce il percorso di acquisizione e gli usi delle lingue che fanno parte del suo repertorio (Pavlenko 2007; Cavagnoli 2014), e si presta particolarmente bene per attività didattiche (Anfosso, Polimeni, Salvadori 2016) che coinvolgono l'intera classe.

\section{Le attività di mentoring}

Le attività menzionate fino a questo punto come buone pratiche sono rivolte sia a coloro che già vivono (o hanno sempre vissuto) nelle società di arrivo che a coloro che sono invece neoarrivati, con l'obiettivo di indurne appunto la riflessione metalinguistica e metaculturale. 
Sono attività caratterizzate da quella che è stata definita «plurilingual resourcefulness lens» (Choi, Najar 2017, 21)

Bezemer and Kress (2016) state, (resourcefulness) refers to 'the resources developed in transformative engagement with the world and accumulated in a community over time' and 'a disposition on the part of members in their use of these resources' (p. 130). By 'plurilingual resourcefulness' we are particularly focusing on the purposeful use of a variety of semiotic resources including multilingual, multisensory and multimodal elements as well as the 'cooperative dispositions' (Canagarajah 2013) that teachers and learners bring and negotiate together for purposes of learning and communication.

Tale pratica, particolarmente usata in contesto didattico ma valida anche al di là di esso, deve necessariamente porsi alla base dei processi di mentoring che, promossi da associazioni che si occupano di inclusione sul territorio o da organismi che gestiscono processi di ricollocamento o resettlement, servono a garantire una migliore integrazione delle persone neoarrivate stimolando al contempo il coinvolgimento dei membri delle comunità ospiti in prima persona. Ma di quali competenze necessita il mentor o la persona che aspira a ricoprire questo ruolo?

Nell'interessante ed esaustiva rassegna proposta da Espinoza Alvarado (2015) l'autore mette in evidenza come lo sviluppo di una competenza pragmatica interculturale e conseguentemente gli interventi didattici da realizzare in tal senso non debbano passare necessariamente attraverso l'individuazione di una norma nativa pragmatica, dal momento che altri fattori che potrebbero concorrere alla diversità nella realizzazione di atti comunicativi da parte dei parlanti non nativi potrebbero essere imputabili a differenti stili comunicativi, a una mancata adesione alle norme socio-pragmatiche e culturali della lingua target, ma soprattutto alla rielaborazione del concetto di norma pragmatica nei parlanti bi e plurilingui.

Negli ultimi anni sono state molte le proposte per migliorare la didattica della pragmatica, data la centralità del tema per un approccio realmente orientato e finalizzato all'azione sociale da parte degli apprendenti. Si vedano ad esempio attività didattiche basate su corpora, quali ad esempio il progetto $L I R A,{ }^{9}$ o quanto proposto in Santoro e Vedder (2016) e Ferrari (2016).

9 Il progetto LIRA (Lingua e cultura italiana in rete per l'apprendimento) ha prodotto una serie di percorsi didattici per l'insegnamento della pragmatica e ha coinvolto quattro diverse università italiane: l'Università per Stranieri di Perugia, capofila del progetto, l'Università di Bologna, l'Università di Verona, l'Università di Modena e Reggio Emilia. Per un approfondimento sui risultati si rimanda a Nuzzo, Zanoni 2012; Zanoni 2017. 
Secondo l'approccio della didattica dei corpora contenuto in Nuzzo, Gauci (2012) e Nuzzo (2016), la proposta di intervento didattico deve basarsi sulla sensibilizzazione di gruppi di apprendenti tramite l'esposizione a modelli di atti linguistici (ad. es. la protesta o la richiesta, tratti da conversazioni spontanee) seguiti da attività di noticing (Schmidt 1990).

Secondo quanto affermato da Chini,

È opportuno incrementare il noticing per favorire non solo l'apprendimento consapevole, ma alla lunga pure l'acquisizione implicita. Come? - ricorrendo a mezzi che migliorano la salienza, prosodica e uditiva o visiva delle forme di L2 (con sottolineature, evidenziazioni foniche, paralinguistiche, o visive nello scritto: input enhancement; Sharwood-Smith 1993), a un livello implicito; - a un livello più esplicito, facendo leva sulla consapevolezza (awareness; Leow 2000; Robinson et al. 2012), tematizzando regole di L2, specie se complesse (tenendo conto dell'età e dello sviluppo cognitivo dei discenti, oltre che della loro L1), sia in modo episodico, in occasione di errori e problemi comunicativi, sia in modo sistematico, tenendo conto di un sillabo orientato in senso acquisizionale; - rivalutare il ruolo giocato da momenti didattici focalizzati sulla forma, stimolando la sua attenzione incidentale verso le forme linguistiche di L2 in momenti a dominanza comunicativa (focus on form), ma anche proponendo attività con focalizzazione esclusiva sulle forme (focus on forms), soprattutto per strutture di L2 marcate o ridondanti. ${ }^{10}$

Per la conversione dell'input in intake, dunque, è opportuno esporre gli studenti a esempi tratti da produzioni autentiche, scritte o parlate, e chiedere loro di osservare e descrivere eventuali comportamenti comunicativi (propri o altrui) insoliti o giudicati inadeguati e attraverso quali espressioni linguistiche vengono realizzati, per poi categorizzare queste ultime per un reimpiego consapevole, sia dal punto di vista interlinguistico che interculturale.

È importante specificare che l'intervento di sensibilizzazione deve tenere conto di una serie di fattori tra i quali la necessità di usare limitatamente il metalinguaggio, la necessità di ricondurre gli atti linguistici sui quali focalizzarsi nel training a situazioni reali della vita quotidiana. Quale migliore occasione quindi per osservare la lingua dal vivo che farlo in situazioni comunicative reali, vicine e che avvengono nella quotidianità?

La proposta didattica qui presentata trae spunto dalle attività realizzate da Università per Stranieri di Siena nell'ambito del progetto

10 Dall'intervento di Marina Chini occasione del workshop Educazione linguistica nelle classi multietniche tenutosi nell'ambito del convegno SLI Lingue in Contatto (Udine, 24-26 settembre 2014). 
COMMIT, ${ }^{11}$ recentemente conclusosi, ed è articolata in due azioni, i cui destinatari sono cittadini neoarrivati (rifugiati resettled) e mentors:

- l'inclusione linguistica dei cosiddetti rifugiati resettled, ${ }^{12}$ ovvero reinsediati tramite corridoi di protezione umanitaria in un Paese diverso da quello in cui è stato chiesto l'asilo. Per facilitarne l'integrazione i governi e le organizzazioni non governative partner offrono servizi come orientamento culturale, formazione linguistica e professionale e programmi per favorire l'accesso ad istruzione e lavoro. L'intervento di formazione pre-partenza (PDO) destinato ai beneficiari di resettlement dovrebbe comprendere una formazione interlinguistico-culturale includendo l'individuazione di un sillabo relativo agli aspetti pragmatici, una sorta di toolkit dal quale estrapolare le situazioni comunicative (da quelle più semplici a quelle che presentano un maggior grado di complessità) nei quattro macrodomini in cui un parlante immigrato può trovarsi ad interagire e in cui è necessario dunque esercitare la competenza comunicativa interculturale e nello specifico gli aspetti pragmatici: dominio personale, dominio pubblico, dominio professionale e dominio educativo (Vedovelli 2012). Un ottimo punto di partenza può essere costituito dal Sillabo per la progettazione di percorsi sperimentali di alfabetizzazione e apprendimento della lingua italiana a livello Pre A1 frutto del lavoro congiunto dei quattro enti certificatori di italiano L2: Università per Stranieri di Siena, Università per Stranieri di Perugia, Società Dante Alighieri e Università degli Studi di Roma Tre. Il sillabo elaborato dai quattro enti appare particolarmente appropriato poiché declina con estrema precisione i bisogni comunicativi di quegli immigrati che presentano un maggior rischio di esclusione sociale, gli immigrati analfabeti o debolmente scolarizzati, verso i quali l'intervento formativo potrebbe risultare più complesso;

- una formazione specifica per i mentors alla competenza comunicativa interculturale. I mentors costituiscono un country specific human capital (Chiswick 1978, 1985 in Månsson, Delander

11 Si tratta di un progetto FAMI recentemente concluso, il cui capofila è l'Organizzazione Internazionale per le Migrazioni (OIM) e di cui l'Università per Stranieri di Siena è beneficiaria (COMMIT: Facilitating the integration of resettled persons in Croatia, Italy, Portugal and Spain). Tra le azioni previste figura il miglioramento della competenza comunicativa interculturale di cittadini immigrati attraverso un'azione di mentoring condotta da cittadini autoctoni o da immigrati ormai ben inseriti nel tessuto sociale della società di accoglienza.

12 Con il termine resettlement (reinsediamento) si intende il processo attraverso il quale «non-EU national or stateless persons who have been identified as in need of international protection to an EU state where they are admitted either on humanitarian grounds or with the status of refugee» (definizione fornita dall'https://ec.europa.eu/ home-affairs/pages/glossary/resettlement-programme_en). 
2017), si tratta infatti di parlanti nativi e migranti già radicati sul territorio che possono agire efficacemente per l'integrazione positiva dei rifugiati nella società di accoglienza attraverso un'azione di mentoring. L'utilizzo del mentoring come strategia per il miglioramento dell' inclusione sociale (e in particolare nei luoghi di lavoro) si è diffuso in diversi contesti basti menzionare il progetto pioniere realizzato in Canada The Mentoring Partnership ${ }^{13}$ mentre altri progetti sono stati sviluppati in Europa come Mentoring for Migrants, realizzato in Austria a partire dal 2008 ad opera della Federal Economic Chamber (WKO) in collaborazione con l'Austrian Integration Fund (ÖIF) e il Labour Market Service (AMS).

In che modo può un progetto di mentoring contribuire a sviluppare la consapevolezza interlinguistica e interculturale? Secondo la definizione contenuta in Bittman $(2011,20)$ «Mentoring is a reciprocal task-related relationship between two people; the so called Mentor and Mentee. It can be considered as training system under which a Mentor is assigned to act as a counselor to a Mentee». In particolare, l'attività dei mentor nei confronti dei cittadini immigrati dovrebbe configurarsi come un'attività di osservazione partecipante scoperta in chiave generativa, ovvero volta «all'instaurazione di un patto etico e cooperativo tra l'osservatore e l'osservato", in modo che lo sviluppo di tale relazione possa portare alla luce aspetti oscuri che dovrebbero dunque emergere in una successiva fase di training. Gli elementi di conoscenza citati dovrebbero dunque emergere in una successiva fase di training, in cui i due protagonisti dell'interazione evidenziano le criticità/i punti problematici secondo una griglia che comprenda i diversi aspetti della comunicazione verbale e non verbale (Benucci 2017).

Nell'esperienza austriaca, in termini di aumento della consapevolezza, l'effetto positivo della relazione interculturale creatasi è stato esplicitamente percepito dagli autoctoni, che dichiarano di aver ridotto notevolmente il pregiudizio nei confronti dei cittadini immigrati e di aver incrementato il senso di partecipazione e responsabilità verso l'integrazione positiva degli immigrati

By creating reciprocal relationships between natives and migrants, the programme considers both; on the one hand the ability of the host society to participate actively in the economic, social and cultural realms of the integration process and on the other hand assures the individual effort of migrants. Mentors having participated in the programme underline especially the positive effect of the intercultural experience, doing away with prejudice and increas- 
ing the sensitivity towards different cultures. Natives actively participating in the mentoring process can add value to their personal as well as professional experiences and in the same token being social responsible by helping foreigners to become an inhabitant of equal value. In the course of the mentoring partnership Mentors can help Mentees to understand, respect and benefit from domestic values, rights and responsibilities. The partnership thereby support the participation of immigrants in the host society on an equal basis and according to EU and Member State laws. The interpersonal relationship of Mentor and Mentee can furthermore contribute to linguistic, civic and cultural awareness of the host society. (Bittman 2011, 56)

Per la formazione dei mentori sono stati redatti, da parte di Università per Stranieri di Siena in collaborazione con OIM, i moduli contenuti nella guida: Percorsi per l'inclusione: Formazione per i mentor di comunità. Competenze comunicative, interculturali, sviluppati nell'ambito del progetto COMMIT per coinvolgere attivamente le società di arrivo nel processo di accoglienza.

\subsection{Corpora e didattica}

Quanto esposto fino a questo momento porta ad affermare che l'aumento della consapevolezza interculturale e interlinguistica è un processo che deve avvenire sia attraverso una spinta top-down sia bottom-up. Da un lato, cioè, le politiche linguistiche e il discorso pubblico devono contribuire all'idea che il plurilinguismo delle società contemporanee - che comprende quindi le lingue di origine dei cittadini immigranti così come le varietà locali, dialetti e lingue di minoranza storica - sia caratterizzato come valore positivo contribuendo a creare un clima favorevole, allontanando idee basate su pregiudizi che vedono i cittadini immigrati e nello specifico i rifugiati come persone 'in deficit' di competenze e come peso per la società; dall'altro, il terreno fertile in cui queste idee possono diffondersi, concretizzarsi e prosperare sono le pratiche didattiche che rafforzano effettivamente queste competenze plurilingue e pluriculturali. Il raggiungimento di questo obiettivo può essere un traguardo concreto, soprattutto se ci si propone di procedere per passi, gradualmente, guidando gli individui all'osservazione dei fenomeni linguistici e culturali che avvengono in interazioni quotidiane.

A tale scopo, è auspicabile proporre attività didattiche che si basino sull'uso di corpora tratti da interazioni reali: come detto, essi presentano il vantaggio di portare dentro l'ambiente classe il mondo, i reali scambi significativi, ed evitano la diffusione di materiali didattici che si basano sulla stereotipizzazione. 
Sebbene infatti l'uso di corpora in glottodidattica sia stato incentivato principalmente per compilare liste di frequenza per tipi testuali diversi, per pianificare materiali di apprendimento per le microlingue, per fornire materiali di riflessione sulle varietà diatopiche, sui cambiamenti diacronici della lingua e su collocazioni e significati (Guidetti, Lenzi, Storchi 2012) o per analizzare i più comuni errori compiuti dagli apprendenti di una L2, con approfondimenti e indicazioni didattiche su problematiche morfosintattiche o di altro tipo apparse particolarmente critiche nell'analisi del corpus (come nel caso del corpus MEDAL, segnalato in Corino 2014), è possibile estenderne le funzioni. Come sostiene Corino, infatti

Usare dei corpora non solo per la ricerca linguistica, ma anche per la formazione di studenti e insegnanti o per l'approfondimento da parte di appassionati di lingue e delle loro mutevoli forme, consente di riflettere davvero su come apprendenti e nativi scrivono, sulla reciprocità degli errori o sulla comunanza di errori fatti ad es. da inglesi e tedeschi o da francesi e spagnoli. È un modo fruttuoso di praticare le nozioni teoriche dei corsi di linguistica generale o italiana (o di linguistica inglese, francese ecc.) e di didattica delle lingue moderne, perché si ha esperienza diretta dei piani linguistici (sempre un po' astratti quando non si vedono concretamente attraverso gli errori) e di cosa vuol dire sovraestendere un morfema o attuare una strategia di evitamento, ci si abitua ad individuare gli errori che sono errori solo in prospettiva transfrastica o solo in termini di accettabilità e ad interrogarsi sul concetto di norma di una lingua... si impara insomma a 'smontare scientificamente' il giocattolo-lingua. (2014, 235)

Questa prospettiva risulta particolarmente interessante giacché lo sviluppo della metariflessività sui fenomeni interlinguistici e interculturali e su come questi due aspetti siano connessi attraverso gli aspetti pragmalinguistici e sociopragmatici della lingua può essere stimolata con risultati entusiasmanti su tutti i parlanti, dal momento che spesso nemmeno i parlanti nativi o più esperti sono consci di determinati aspetti pragmatici della lingua target (Cortés Velásquez, Nuzzo 2017). Per trattare gli aspetti culturali e interculturali, veicolati principalmente attraverso gli aspetti diafasici della lingua, attraverso l'uso dei corpora, il docente deve certamente acquisire una funzione di guida alla scoperta dei fenomeni della lingua-cultura da parte di un apprendente protagonista attivo, nell'ottica del già citato noticing. Le attività didattiche devono basarsi innanzitutto sullo sviluppo della capacità di comprensione del testo e del contesto, naturalmente declinate secondo coordinate (inter)culturali, proprio per decodificare l'adeguatezza della lingua su tutti i livelli: di registro, lessicale, morfosintattico. Un'attività didattica, ad es. basata 
su dati tratti da un corpus e destinata sia a studenti (nello specifico rifugiati) che a membri esperti della società di accoglienza può essere usata come valido strumento per l'accrescimento della competenza interlinguistica e interculturale. Si analizzerà ora un esempio (esempio 4) che può essere usato in contesto didattico a questo scopo. Lo stralcio che segue è tratto dall'interazione sul luogo di lavoro tra Hernan, peruviano, e Hasan, somalo. I due lavoratori devono concordare una pausa durante il turno di lavoro. Si è ritenuto importante inserire proprio questo esempio perché lo small talk, sebbene non risponda a obiettivi di literacy funzionale per i cittadini immigrati, costituisce la base attraverso la quale possono essere costruite le relazioni umane, con l'obiettivo non appiattire sulla dimensione professionale la complessità interazionale (Roberts, Cook 2009 in Choi, Najar 2017). Inoltre, in studi recenti basati su un approccio partecipativo, che coinvolge quindi gli apprendenti in un percorso attivo di definizione dei propri bisogni, la capacità di reagire in maniera appropriata alle sollecitazioni in contesti socio culturali quotidiani era una delle risposte fornite dalle donne rifugiate destinatarie del corso di lingua. L'atto linguistico al centro di questa interazione, il rifiuto, è un atto linguistico reattivo e dispreferito, fornito in risposta ad un altro atto, come ad es. un invito; esso risulta particolarmente difficoltoso in un'interazione giacché espone a una minaccia della faccia entrambi gli interlocutori (De Marco 2021); a livello socio-pragmatico risulta particolarmente ostico per gli apprendenti di una L2, in particolare per la difficoltà a produrre strategie di mitigazione del rifiuto, anche perché essi non vengono esposti sufficientemente a input autentici, come quelli che possono invece offrire i corpora.

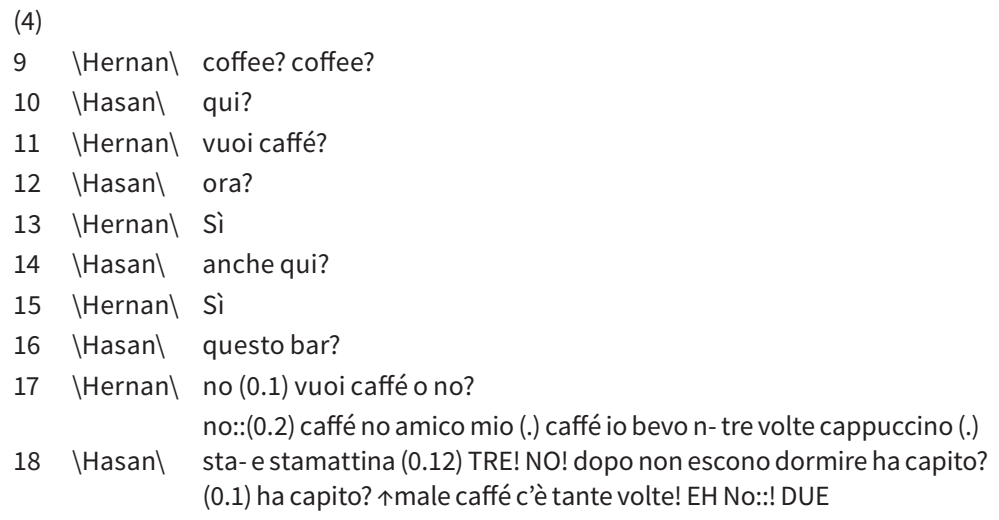




\section{Pausa caffè}

\begin{tabular}{|c|c|}
\hline Attività 1. & $\begin{array}{l}\text { In che modo Hernan ha invitato il collega a prendere il caffè? Quali } \\
\text { lingue ha usato? Secondo te l'invito è chiaro? Cosa avrebbe potuto } \\
\text { dire? }\end{array}$ \\
\hline Attività 2a & $\begin{array}{l}\text { Secondo te Hasan aveva delle difficoltà a rifiutare l'invito del suo } \\
\text { collega a prendere un caffè insieme? Alla fine accetta o rifiuta } \\
\text { l'invito? Quali parole usa per rifiutare? Quale è il motivo del rifiuto? }\end{array}$ \\
\hline Attività 2b & Secondo te il collega Hernan si è innervosito? Perché? \\
\hline \multirow[t]{4}{*}{ Attività 3.} & $\begin{array}{l}\text { Prova a immaginare cosa avrebbe dovuto dire Hasan nelle seguenti } \\
\text { situazioni: }\end{array}$ \\
\hline & $\begin{array}{l}\text { a. Il capo gli ha chiesto di cambiare un turno con un collega per } \\
\text { questa settimana. Hasan non vorrebbe perché il giorno richiesto } \\
\text { è il compleanno di suo figlio. Cosa dice Hasan? }\end{array}$ \\
\hline & $\begin{array}{l}\text { b. Il collega di Hasan gli chiede in prestito la macchina per il giorno } \\
\text { dopo visto che la sua è rotta, ma anche se Hasan usa il motorino, } \\
\text { non può prestargliela perché la moglie la usa per andare al } \\
\text { lavoro ogni mattina. Hasan? }\end{array}$ \\
\hline & $\begin{array}{l}\text { c. L'amico di Hasan vuole invitare Hasan a pranzo domenica, ma } \\
\text { Hasan e la sua famiglia non possono andare. Cosa dice Hasan? }\end{array}$ \\
\hline
\end{tabular}

Le prime tre attività (1, 2a e 2b) sono incentrate sul riconoscimento del contesto e delle strategie comunicative usate dai due interlocutori. Le finalità didattiche sono chiare: elicitare il riconoscimento della funzione comunicativa in relazione agli aspetti pragma-linguistici e socio-pragmatici, sia per l'invito che per il rifiuto; far osservare agli studenti l'uso di tutte le risorse linguistiche usate dagli interlocutori per raggiungere la comprensione reciproca. L'attività (2b) invece ha l'obiettivo di proporre un esercizio di 'emergent listening', ovvero la possibilità di creare una connessione con l'interlocutore attraverso un ascolto attivo ed empatico.

Attraverso una proposta del genere, si perseguono gli obiettivi didattici sfruttando esempi di lingua viva, che possano testimoniare situazioni comunicative reali in cui i parlanti possono trovare, ed osservando da vicino le dinamiche interazionali, apprezzandone gli aspetti interlinguistici e interculturali e facendone oggetto di riflessione.

Per concludere con le parole di Canagarajah e Liyanage $(2012,58)$ «what we need is a paradigm shift in language teaching. Pedagogy should be refashioned to accommodates to the modes of communication and acquisition seen outside the classroom». Un cambio di prospettiva offerto dalla ricerca è quindi auspicabile nella didattica delle lingue perché il potenziale plurilingue possa essere evidenziato e usato in tutta la sua ricchezza per sviluppare consapevolezza interlinguistica e interculturale. 


\section{Aspetti psicologici della migrazione e gestione dei conflitti}

Viola Monaci

Università per Stranieri di Siena, Italia

Sommario 4.1 Vissuti traumatici e conseguenze psicologiche. - 4.2 Vulnerabilità e questioni di genere. - 4.3 Aspetti psicologici legati all'acquisizione della L2.

\subsection{Vissuti traumatici e conseguenze psicologiche}

L'Occidente ha subito un cambiamento sia in termini quantitativi che qualitativi sul piano della demografia negli ultimi trent'anni. Una realtà in evoluzione continua a partire dai primi fenomeni migratori verso i Paesi colonizzatori, fra il 1500 e il 1700, chiamata in letteratura 'migrazione post-coloniale' che alla fine del 1800 ha visto l'assetto di molte popolazioni nei vari Paesi. L'obiettivo primario di questi migranti era quello di cercare fortuna nei Paesi che erano stati conquistatori, mentre successivamente questo fenomeno è mutato in termini di organizzazione, direzioni, esigenze, bisogni e motivazioni.

La migrazione è un atteggiamento che gli esseri umani hanno adottato nel corso della storia per insediarsi temporaneamente o stabilmente in un luogo diverso da quello di origine. Come già accennato nel capitolo 2 parlando di migrazione umana si può fare riferimento ad un fenomeno sociale dovuto a diversi fattori che ha come obiettivo quello di migliorare le proprie condizioni di vita.

Volendo dare una definizione di base di migranti si può dire che sono persone impegnate in fenomeno di movimento umano (da qui 
in avanti FMU). Gli FMU spostano una quantità ingente di persone ogni giorno con varie modalità, e la migrazione è uno di questi. Questi fenomeni hanno portato in Europa persone provenienti da realtà culturali e in situazioni sociali di appartenenza eterogenee.

La psicologia delle migrazioni si occupa di studiare gli individui impegnati in un FMU, distinguendo la migrazione in «migrazioni economiche» e «migrazioni forzate», un fenomeno che non sempre è riconducibile ad una migrazione 'volontaria', ma piuttosto ad una 'migrazione forzata' che si distingue dalla prima per l'influenza di diverse condizioni. Nel primo caso, i migranti spesso si trovano a condividere un «drammatico passato traumatico, minaccioso per la integrità e la continuità psichica, che include l'esposizione a violenza correlata con la guerra, aggressione sessuale, tortura, incarcerazione, genocidi e altre forme di minacce e annichilazione personale» (Friedman, Jaranson 1994, 4), persone costrette ad abbandonare il proprio Paese di origine in quanto vittime di violenze, umiliazioni, minacce, persecuzioni; nel secondo caso, invece, finalizzata al miglioramento della propria condizione socio-economica. Da studi condotti emerge che coloro che nel proprio percorso di vita subiscono soprusi, per motivi spesso riconducibili alla razza, religione, nazionalità, gruppo sociale o opinioni politiche, sono persone che si trovano costrette ad abbandonare il proprio Paese e le famiglie in cerca di protezione internazionale e manifestano problematiche di salute fisica, mentale e in alcuni soggetti sintomi del Disturbo Post Traumatico da Stress (PTSD). ${ }^{1}$

Tutti fattori che sono riconducibili ad eventi traumatizzanti nella vita di un migrante e che come anche sottolineato da Grosso (2021, 27), «i traumi psicologici subiti, la vita in campi profughi o in contesti disagiati, la separazione dalla famiglia, possono rendere l'adattamento dei rifugiati a una nuova società un processo estremamente complesso e delicato, per il quale è necessaria un'adeguata preparazione». Gli spostamenti vengono vissuti dal migrante come vere e proprie fughe che possono rivelarsi eventi traumatizzanti per il loro vissuto in condizioni di insicurezza, precarietà e rischio, affiancati dalla perdita della propria condizione economica e sociale, dei legami affettivi ecc. Ogni essere umano è un'entità coinvolta in un sistema sociale che lo ingloba, e il disorientamento dovuto alla perdita della matrice sociale ha gravi effetti a lungo termine sia sul funzionamento sociale, sia su quello psicologico e biologico (cf. Van der Kolk 2004).

Molti sono gli studi che si sono occupati di approfondire le conseguenze della migrazione in termini di disturbi psicologici: uno di questi, svolto su un campione di rifugiati siriani in Turchia, ha por-

1 Si veda ad esempio il report Mental health disorders in asylum seekers and migrants overlooked by inadequate reception system (MSF 2016), https://www.msf.org/italymental-health-disorders-asylum-seekers-and-migrants-overlooked-inadequate-reception-system. 
tato alla luce l'importanza della continuità dell'identità sociale come fattore di protezione della salute mentale. Gli studiosi Danon e Miltenburg, nel 2001, si sono occupati nella loro ricerca di trattare queste tematiche affrontando proprio il problema del trauma migratorio che, a loro parere, è caratterizzato da viaggi lunghissimi e drammatici, malnutrizione, malattie non curate, aggressioni, morte dei compagni di viaggio, sfruttamento, violenze di diverso genere; inoltre, spesso accade che i Paesi di frontiera detengano le persone per lungo tempo in campi profughi o li respingano violando la Convenzione di Ginevra (cf. Benvenuti 2006).

Volendo spostare l'attenzione sul contesto italiano, risultano essere in aumento i casi di disagio mentale di richiedenti asilo e rifugiati che prendono parte ai progetti SPRAR (Sistema di protezione per richiedenti asilo e rifugiati) e CARA (Centri di accoglienza governativi per richiedenti asilo), e che manifestano la loro vulnerabilità nel periodo di accoglienza. Questo rappresenta il primo contatto fra le due parti che risulta essere di fondamentale importanza per una prima diagnosi dei casi affetti da disagio mentale e che può servire per l'avviamento di percorsi terapeutici o programmi di protezione della salute mentale. Il programma annuale 2014 del Fondo Europeo dei Rifugiati, ad esempio, prevede azioni volte alla tutela dei soggetti vulnerabili, con misure di supporto e di riabilitazione di vittime di violenza e torture o portatori di disagio mentale, così come descritto all'interno del Rapporto:

il quadro sintomatologico più frequentemente riscontrato è prevalentemente caratterizzato da intrusioni diurne e notturne (ricordi e incubi angoscianti del trauma subito) con associate reazioni emotive e fisiche, disturbi del sonno, della memoria, dell'attenzione e della concentrazione. (SPRAR 2010, 7)

Pertanto, a livello internazionale, è richiesta l'implementazione di programmi atti a proteggere la salute mentale dei richiedenti asilo e rifugiati e di interventi volti a potenziare le abilità di riconoscimento e gestione del loro disagio psichico da parte degli operatori.

Secondo numerose pubblicazioni, fra le quali il rapporto 2020-21 di Amnesty International su La situazione dei diritti umani nel mondo, tipologie varie di violenza vengono praticate in numerosi Paesi del mondo.

Lorenzo Tarsitani, psichiatra dell'Università la Sapienza di Roma, in un'intervista rilasciata per il canale 1c1y SOPSI 2020, chiarisce quella che è la realtà del sostegno psicologico a migranti che risultano avere un estremo bisogno di essere supportati socialmente e della presenza di un 'intreccio' fra le condizioni di vita post migratorie e la sofferenza mentale. Si sta parlando di persone che si trovano ad affrontare un momento delicato del loro percorso di vita nei centri 
di accoglienza straordinaria, nei circuiti SPRAR, che non risultando residenti, in un ipotetico arrivo in ospedale, non possono essere reindirizzati ai servizi sociali e nemmeno ai servizi di salute mentale di comunità. A tal proposito lo psichiatra sottolinea l'esigenza di istituire servizi sociali ulteriori che non risultino essere territoriali (legati alla residenza). I pazienti che manifestano disturbi mentali, se hanno una condizione caratterizzata da un'elevata mobilità risultano difficili da seguire, e risulta complicato anche programmare progetti terapeutici ad hoc (visite, controlli follow up).

La psicologia e la psicopatologia delle migrazioni, sotto gli aspetti fenomenologici, eziologici, di diagnosi e trattamento sono da considerarsi un settore della psicologia e della psichiatria che in futuro avrà una grande importanza.

Fra le ragioni che rendono necessario lo sviluppo di queste branche della psicologia si annovera la difficoltà di fornire una definizione di 'soggetto migrante': spesso questo risulta essere un concetto complesso e confuso, tanto da essere compattato in una semplificazione estrema. Questa visione monolitica e confusa non permette di poter affrontare correttamente il problema del disagio sociale/mentale e dei disturbi dei migranti. Se per gli operatori i pazienti migranti rimangono un unico contenitore addensato non si può agire per il loro bene. Le ragioni di questo stato delle cose sono diverse. Nonostante l'argomento sia al centro di un dibattito nazionale e internazionale, e nonostante esistono delle buone pratiche o interventi nei diversi ambiti territoriali, ancora oggi persistono una ignoranza e una lentezza della società e delle istituzioni nell'utilizzare la lente della psicologia delle migrazioni.

Il paradigma bio-psico-sociale viene utilizzato oggi come modalità per capire l'essere umano e considera importanti anche gli aspetti contestuali in cui esso vive, le relazioni sociali e gli aspetti culturali, linguistici e tecnologici che risultano rilevanti nei processi di patologia dei disturbi mentali (social and cultural neuroscience).

L'approccio della psicologia classica vede la mente dell'individuo come sede della spiegazione di tutto. Questo approccio ha creato però nella storia, e tramandato, numerose imprecisioni e stereotipi non scientifici riguardo ai migranti. Una visione che giudicava l'individuo migrante come colui che si sposta classificandolo come 'soggetto incapace di risiedere' perché disturbato, instabile, marginale e disadattato.

La psicologia delle migrazioni adotta una prospettiva ecologista, che si differenzia dall'approccio classico della psicologia intra-soggettiva, perché prende in considerazione anche le spiegazioni contestuali, oltre che avvalersi dell'avanzamento dalle neuroscienze, delle competenze delle scienze sociali, culturali, storiche ed economiche per spiegare le ragioni degli individui che sono impegnati nel fenomeno della migrazione. 
La migrazione è una nuova frontiera e per i clinici una sfida al sapere precostituito perché non tutti i migranti attraversano le stesse esperienze, o si situano in contesti sociali simili: essi non preparano il loro viaggio allo stesso modo e non sono mossi dalle stesse ragioni.

È il processo migratorio stesso e i conseguenti aggiustamenti culturali e sociali ad avere un ruolo sulla salute mentale dell'individuo, le classificazioni sono solo delle semplificazioni. Tuttavia, esse aiutano ad inquadrare i fenomeni.

A tal proposito, è giusto specificare che i migranti si possono suddividere in due differenti categorie:

- coloro che compiono il viaggio;

- coloro che da loro discendono (figli e nipoti).

Naturalmente tutto ciò è una semplificazione che riprende e chiarisce alcuni concetti della psicologia dei migranti e la fenomenologia dei loro disagi e disturbi.

Oltre alle competenze psicologiche e psichiatriche in futuro saranno sempre più richieste la competenza culturale, transculturale, la conoscenza fenomenologica, l'esperienza in contesti estremi e di emergenza, la capacità di lavorare in équipe interculturali.

Il campo della psicologia delle migrazioni è un settore specifico e prevalentemente esplorato dagli operatori, da quello che riescono a compiere quando incontrano gli stranieri, spesso senza avere la possibilità di fruire di corsi di preparazione specifici che permetterebbero di collegare l'esperienza del singolo migrante al contesto familiare e sociale dal quale proviene. Il fenomeno migratorio è infatti molto complesso perché parliamo di un reale investimento non soltanto del singolo, ma dell'intera famiglia; inoltre, mettere a contatto l'individuo con la sua vulnerabilità diventa potenzialmente traumatico, e in questo trauma si trovano aspetti legati al viaggio e ai metodi che si utilizzano per compiere il percorso. Inoltre, le condizioni psicologiche legate allo sradicamento e al cambio di abitudini, il confronto con una nuova cultura e condizioni ambientali, determinano la comparsa di sindromi specifiche migratorie, che vanno a modificare eventuali sindromi pregresse.

Ad oggi, l'interesse per questi studi e per le popolazioni migranti è ridotto a causa della miopia e del ritardo concettuale degli istituti di formazione e degli operatori della salute mentale stessi. Queste evidenze hanno più di una ragione in quanto la maggior parte di questi studi sono di carattere storico e non di interesse scientifico. Gran parte di questi hanno riguardato richiedenti asilo cubani in USA o studi sui ghetti multiculturali pieni di colonie di migranti nelle metropoli europee in Inghilterra, Olanda e Svezia.

Ci sembra opportuno a tal proposito citare alcune delle ricerche europee svolte in ambito migratorio e sulla salute mentale nei richiedenti asilo, persone che hanno perseguito la strada della migrazione forzata. 
«Negli ultimi trenta anni numerose sono state le crisi umanitarie, conseguenza diretta di guerre e genocidi, che si sono succedute nel tempo e che hanno causato milioni di sfollati e rifugiati in tutto il mondo» (SPRAR 2010, 5): secondo la rassegna dell'Alto Commissariato delle Nazioni Unite per i Rifugiati, sono 82,4 milioni le persone in fuga nel mondo. Possiamo parlare quindi di migrazioni forzate e di massa che espongono ancora di più queste persone a conseguenze dannose per la loro salute mentale. L'Italia è il secondo Paese in Europa per ingresso per i richiedenti asilo dopo la Grecia, dopo il transito in Libia.

Comunemente le condizioni mediche dei migranti vengono valutate all'arrivo bypassando lo stato di salute mentale che non viene in alcun modo valutato, nonostante la probabilità di riscontrare gravi traumi prima e durante la migrazione. Alcuni strumenti per la valutazione della salute mentale e la cura psicologica dei richiedenti asilo sono stati di recente elaborati dall'ONG Medici senza frontiere in accordo con il Ministero della Sanità in contesto siciliano. Grazie a questo è stato portato alla luce uno studio su questa tipologia di migranti arrivati a Lampedusa tra il 2014 e il 2015, per la documentazione delle condizioni di salute mentale, di eventi traumatici e di difficoltà di vita post migratorie (Crepet et al. 2017). Uno screening di salute mentale effettuato su 385 persone ha evidenziato che il 50\% (193) sono affetti da disturbi della salute mentale. Il campione dei monitorati rispondeva alla figura del giovane maschio proveniente dall'Africa Occidentale partito dal proprio Paese di origine un anno prima dell'arrivo. Tra le diagnosi più comuni sono risultate per il 31\% disturbo post traumatico da stress e il 20\% depressione. Questi disturbi, che risultano essere conseguenze di eventi traumatici riscontrabili già dal Paese di origine (60\%) e durante il percorso migratorio (89\%) sono dati da situazioni di conflitto e/o rischio di morte dopo aver assistito a episodi di violenza o di essere stati detenuti. Gli episodi di inattività, di preoccupazione per la famiglia e per la casa di origine, la sensazione di solitudine e il terrore di dover tornare nel luogo dal quale sono fuggiti, risultano essere le prime difficoltà riscontrate dai richiedenti asilo nei centri di prima accoglienza. Questi studi ci suggeriscono che i servizi di sostegno psicologico e sociale dovrebbero far parte dei percorsi di accoglienza dei richiedenti asilo integrandosi ai servizi già attivi sin dai primi momenti al fine di contrastare le condizioni di disagio psichico presenti nella fase pre-migratoria, eventi traumatici e di sopperire alle difficoltà di vita che si possono comunemente ritrovare nella fase post-migratoria.

Un ulteriore studio condotto da Lolk et al. (2012), basato sulla presenza di disturbi concomitanti tra quelli somatici, da stress post traumatico e depressione, è stato rilevato in un campione di migranti in Danimarca. I soggetti della valutazione hanno ottenuto il permesso di soggiorno in Danimarca dal 1993 al 2010 (ca. 92.000 persone). I 
risultati hanno mostrato che coloro che sono affetti da PTSD e depressione risultavano avere un tasso più elevato di malattie somatiche a differenza di coloro che risultavano privi di disturbi psichiatrici. Questi risultati hanno portato alla luce la necessità di servizi di prevenzione e di cura per il miglioramento della salute generale dei migranti. Pertanto, il numero sempre più crescente di rifugiati e di richiedenti asilo e migranti irregolari risulta tutt'oggi essere una sfida per i servizi di salute mentale europei.

Un'indagine (Priebe, Giacco, El-Nagib 2016) ha fatto emergere che tutte le categorie di migranti sono esposte a fattori di rischio con conseguenti disturbi mentali, prima, durante e dopo il percorso migratorio. Il numero di disturbi rilevato tra i gruppi di migranti è variabile ma complementare a quelli delle popolazioni ospitanti, tranne che per i disturbi post traumatici da stress che nei primi risulta avere un tasso maggiore. La depressione e i disturbi da ansia sono conseguenze del tempo e delle condizioni socioeconomiche instabili, oltre che della mancanza di integrazione sociale e della disoccupazione. Secondo la letteratura il fattore di rischio più comune per l'insorgenza delle psicosi è la migrazione stessa (Bhugra 2004; Cantor-Grae, Selten 2005). Nella Comunità sud Asiatica presente in Gran Bretagna è stato rilevato un elevato fattore di psicosi rispetto alla Comunità nativa (Bourque, Van Der Ven, Malla 2011).

Questi gruppi di persone incontrano spesso ostacoli all'assistenza sanitaria per cui il lavoro dei professionisti viene svolto in termini di riduzione del danno psicologico.

Mentre il trauma nella fase pre-migratoria viene riconosciuto come un fattore scatenante dei danni di salute mentale nei rifugiati e nei richiedenti asilo, la ricerca si è quindi poi concentrata sugli effetti psicologici dell'impatto dell'immigrazione e quindi all'arrivo nell'ambiente di accoglienza. Per questo motivo, un ulteriore studio (Steel et al. 2017) ha cercato di fornire dati sui traumi pre-emigrazione, post-migrazione e sulle conseguenze psicologiche su immigrati e rifugiati provenienti dall'Africa e diretti in Svezia. Il campione di indagine, composto da 420 rifugiati e immigrati, è stato interamente sottoposto ad un questionario fra cui l'Harvard Trauma Questionnaire, la Scala Difficulties Living Post-migration, il Questionnaire del Lifestyle Cultural e la Checklist di Hopkins. Dai risultati raccolti, è emerso che l'80\% dei partecipanti ha vissuto almeno un'esperienza traumatica prima dell'emigrazione, il $44 \%$ ha mostrato di soffrire di PTSD e il $20 \%$ ha dimostrato sintomi depressivi. Gli uomini hanno evidenziato un maggiore fattore di stress post-migrazione rispetto alle donne, che invece hanno riportato una maggiore sofferenza di sintomi depressivi.

Gli elementi considerati condizionanti nella persona migrante sono la salute post-immigrazione e gli aspetti socioculturali come il grado di alfabetizzazione ottenuto nel Paese ospitante. Dato inte- 
ressante emerso è l'alta percentuale di PTSD in coloro che si trovano in Svezia da minore tempo, sintomo questo aggravato da numerosi eventi traumatici.

Una ulteriore ricerca realizzata da Li, Liddell e Nickerson (2016) ha confermato che i rifugiati riportano sintomi di PTSD e di altre tipologie di disturbo in relazione allo stress post-migrazione e alla salute mentale, portando ancora una volta alla luce come i fattori connessi ai processi di integrazione e le politiche di immigrazione, compresi quindi quelli socioeconomici, sociali e interpersonali, danneggiano l'assetto psicologico dei rifugiati.

Uno studio recente (Guardia et al. 2016) ha fornito dati sulla presenza di disturbi mentali nei migranti di prima, seconda e terza generazione ed ha confermato che il fenomeno migratorio costituisce un elemento di rischio psicopatologico. Psicopatia che, come riportato in letteratura, insorge a seguito dell'esperienza migratoria (Lastrina 2017), come in Gran Bretagna, nelle comunità sud-asiatica e caraibico-africana, dove il tasso di psicosi è più elevato rispetto alla popolazione nativa.

I dati di tutte le ricerche esaminate confermano che i fattori di stress post-migratorio legati all'ambientamento e all' integrazione sono stati i principali correlati della salute mentale negli immigrati umanitari. Diventa necessario quindi programmare interventi di prevenzione e di riduzione del danno (programmi di assistenza psicosociale e psicologica), come avviene in Germania dove si è iniziato a strutturare interventi in questo ambito (Erim, Morawa 2016).

La sfida per gli Stati membri dell'Unione Europea risulta essere duplice, da una parte per l'integrazione sociale dei rifugiati, dei richiedenti asilo e degli immigrati irregolari dei Paesi ospitanti, dall'altra delle buone pratiche per il miglioramento dell'accesso e dei percorsi di cura della salute mentale.

Per questo sarebbe opportuno promuovere strategie di attuazione delle politiche che raggiungano l'obiettivo di affrontare e prevenire disturbi mentali, fornendo risorse per l'integrazione ed inclusione sociale, per la diffusione di informazioni sui diritti e servizi disponibili e che forniscano offerte formative per personale specializzato nel settore. Di fondamentale importanza sarebbe il monitoraggio degli interventi proposti, nello specifico sulla fruizione dei servizi e sui risultati raggiunti, una valutazione delle attività da vari punti di vista e la flessibilità organizzativa per coordinare al meglio gli interventi necessari.

Per concludere questo primo aspetto, è opportuno evidenziare che sulla base dei fattori di rischio precedentemente analizzati, che rendono le persona migranti estremamente fragili, esponendole ad un'alta possibilità di manifestare disturbi mentali, è necessario implementare percorsi che coinvolgano team di professionisti del settore come etnopsichiatri, etnopsicologi, medici, mediatori linguistici-culturali, 
che possano affrontare la questione ed intervenire uniformemente in vista di un solo ed unico obiettivo. I beneficiari di questi interventi non possono essere solo i diretti interessati della migrazione, ma anche le generazioni successive, poiché i fattori di rischio per il disagio psichico non si esauriscono alla prima generazione, ma possono avere ripercussioni fino alla terza generazione (Grinberg, Grinberg 1990).

\subsection{Vulnerabilità e questioni di genere}

In vista di una corretta gestione dei flussi migratori nel contesto europeo è importante prendere in esame alcune variabili sociodemografiche come il genere, un dato importante nell'ambito delle politiche migratorie. Alcuni studi demografici hanno evidenziato come motivazione migratoria la scelta dell'identità di genere, che influenza in modo significativo il processo migratorio, le relazioni e le dinamiche interne. Altri aspetti influenzati dal genere sono i rischi, le vulnerabilità e i bisogni, che cambiano tra i vari gruppi. Una stima dimostrata dall'European Institute for Gender Equality (EIGE) afferma che il 7\% della popolazione totale europea è nata al di fuori dei confini e la metà di questa sono donne e ragazzi. Secondo questi recenti studi le motivazioni che spingono queste persone a migrare sono il lavoro, lo studio, il ricongiungimento familiare, lo status economico e tutte le forme di persecuzione. La migrazione è vista come un'opportunità per le famiglie che si trovano a far parte di quelle società in cui non esiste libertà di espressione e decisionale, soprattutto per le donne. «La possibilità di potersi spostare da un Paese all'altro e di potersi rimettere in gioco da soli con sé stessi, se è un atto volontario è, responsabilizzante» (Borrelli 2020), che può indurre un cambiamento in primis nelle donne ma anche nelle società di origine e di accoglienza.

Durante il processo migratorio le donne si trovano a dover affrontare dei rischi e delle situazioni di insicurezza diverse da quelle degli uomini, mostrandosi fortemente vulnerabili e soggette a violenza di genere (sfruttamento sessuale e matrimoni forzati).

La rappresentazione data dai media di migranti come vittime, soggetti vulnerabili è negativa e può quindi alimentare episodi di razzismo, di xenofobia e stereotipi verso l'oppressione delle donne migranti. Una prima differenza di genere si riscontra sul piano del mercato del lavoro, in quanto le donne risultano essere in numero maggiore disoccupate o economicamente svantaggiate. Tuttavia, l'inserimento nel mondo del lavoro è influenzato dal motivo della migrazione: le donne che migrano per motivi familiari hanno dei tassi di occupazione più bassi rispetto a quelle che arrivano per motivi di studio o di lavoro.

Una peculiarità della migrazione delle donne è la non percezione del progetto migratorio come una scelta propria, ma piuttosto vinco- 
lata. La stesura del progetto viene adottata da un familiare che può essere già immigrato, da un'amicizia della famiglia oppure da membri della comunità. Un altro aspetto interessante sul piano decisionale delle donne viene esplicitato da Edelstein (2003) in un suo articolo:

La decisione però spetta a loro e questo è un momento significativo, strettamente collegato alla 'benedizione della mamma'. Infatti, le donne dichiarano che il consenso della madre diventa basilare nel processo decisionale riguardante la propria migrazione. Molte di loro sottolineano che se non avessero ottenuto l'approvazione materna, avrebbero rinunciato o non avrebbero osato. Questa posizione sembrerebbe dovuta in parte al fatto di dover talvolta lasciare i figli con le nonne, ma soprattutto al bisogno di mantenersi collegate al rapporto che le donne mantengono con la madre anche da adulte. (Edelstein 2003)

Le donne concentrate sui contesti familiari che lasciano nel Paese di origine, non si creano aspettative sul Paese di destinazione. Gli unici pensieri riguardano esclusivamente l'aspetto economico del loro futuro, il miglioramento della loro vita e la fuga da una situazione politica pericolosa. Molte di loro vivono la partenza come una separazione a tutti gli effetti e una chiusura totale con la vita che conducevano prima, altre invece non danno molta importanza ad una fase che può permettere loro di voltare pagina.

Le donne adagiate in una posizione iniziale di non potere decisionale, si affidano ad un progetto altrui, togliendosi di dosso ogni senso di responsabilità. Quando si verificano situazioni complicate e di disagio, la donna talvolta fa ricadere ogni colpa su chi ha progettato lo spostamento. Altresì, se il progetto ha successo, se ne sentono le protagoniste e ne prendono orgogliosamente parte.

L'arrivo nel Paese di destinazione crea in loro grandi aspettative nei confronti del progetto stesso e della nuova vita che le aspetta. Un insieme di stimoli che inebriano la loro anima di euforia, entusiasmo e ottimismo, come ogni novità che un essere umano si trova a dover affrontare. Ciò che invece alcune volte si verifica è uno stato d'animo di depressione, apatia o totale indifferenza dovuto ad ostacoli, difficoltà o conflitti con le società di accoglienza, nel lavoro o nelle famiglie. Questa fase che si può definire di transizione che comporta quindi delle crisi personali dipende molto anche dalle aspettative e dai bisogni che si manifestano nella fase di adattamento. Socializzare, creare delle reti di rapporti con persone locali o connazionali, definire un ruolo nella famiglia nella società e definirsi nei confronti della popolazione è una fase di inserimento che può suscitare le prime difficoltà nel Paese di arrivo. Solo dopo alcuni anni molte donne riescono a capire realmente qual è il loro ruolo all'interno della nuova società, percepiscono i primi cambiamenti 
nelle relazioni con gli altri, fino a sviluppare in sé stesse una forte autostima che le rende donne più sicure, ma soprattutto padrone e protagoniste della propria vita. Affiancata a ciò c'è però una piccola fase di difficoltà e un senso di estraniamento dovuto al dover far percepire il vero cambiamento alla famiglia nel Paese di origine, che spesso le giudica.

Da una ricerca svolta attraverso l'utilizzo del metodo narrativo (Edelstein 2002) e all'interno di percorsi di gruppo con donne migranti (Edelstein 2000) è stato notato che un aspetto di forte interesse è quindi l'idea del ritorno nelle donne migranti che quindi da loro la forza per poter vivere al meglio l'esperienza migratoria. Dalle narrazioni raccolte e analizzate di donne straniere «è interessante notare che quando si chiede come e quando intendono tornare nel Paese d'origine si ottengono risposte quasi invariabilmente vaghe e riguardanti progetti lontani: pensione, figli sistemati» (Edelstein 2000, 71).

Alcuni studiosi dei processi migratori hanno rilevato l'importanza dei momenti che precedono l'arrivo nel Paese di destinazione e come questi condizionino l'adattamento e l'integrazione.

Sluzki (1979) nei suoi studi mostra le fasi del fenomeno migratorio dal punto di vista familiare e sistemico. Il focus è posto sui pattern di comunicazione che si manifestano nella famiglia migrante durante il processo migratorio e ne propone un modello culture free, privo cioè del bisogno di individuare elementi culturali. Le cinque fasi sono collegate fra di loro, solo le prime due riguardano i momenti prima dell'arrivo, mentre le tre successive la permanenza nel Paese di accoglienza:

- la prima fase è quella legata alla famiglia e ai preparativi con scambi di lettere, richiesta di visto, incontri con amici o parenti emigrati;

- la fase di partenza, e l'atto stesso, può essere molto breve come un viaggio in aereo oppure molto lunga e travagliata con spostamenti nei Paesi di transizione;

- la fase dopo l'arrivo nel Paese di destinazione, che può essere di settimane o mesi, definita di 'ipercompensazione' vede le famiglie impegnate nel soddisfare i bisogni senza contare lo stress del viaggio migratorio;

- la fase chiamata di 'decompensazione' che prevede momenti difficili di crisi.

Gli aspetti psicologici della migrazione femminile e maschile si differenziano. In primis la percezione di compiere un percorso che non sentono proprio, per le prime, in quanto non direttamente voluto da loro ma imposto dall'uomo, nonostante la tradizione femminista insegni che esiste la possibilità di scegliere ed essere artefici responsabili della propria vita, considerando questo un pilastro del benessere e la chiave per riuscire nei propri progetti di vita (Irigaray 1992). 
Gli uomini migranti dichiarandosi responsabile del progetto migratorio e promettendo vita migliore nel futuro prossimo a famiglie e comunità, generano aspettative che spesso finiscono per deludere e li rendono vulnerabili, aspetto questo che non tocca la figura femminile che non sente questo senso di responsabilità. Nella tabella che segue si riportano alcuni aspetti salienti dei percorsi migratori al maschile e al femminile.

Tabella 1 Tabella esemplificativa sulla differenza dei processi migratori di donne e uomini

\begin{tabular}{|c|c|}
\hline Processo migratorio al femminile & Processo migratorio al maschile \\
\hline $\begin{array}{l}\text { 1. Progetto antico / esperienza } \\
\text { lontana. }\end{array}$ & $\begin{array}{l}\text { 1. Progetto antico / esperienza } \\
\text { lontana. }\end{array}$ \\
\hline 2. Progetto concreto (di un altro). & 2. Progetto concreto e decisione \\
\hline $\begin{array}{l}\text { 3. Propria decisione - consenso } \\
\text { materno. }\end{array}$ & (nronri) \\
\hline $\begin{array}{l}\text { 4. Commiato (tristezza, pianti, rituali). } \\
\text { Sguardo indietro. }\end{array}$ & $\begin{array}{l}\text { 3. Preparativi (entusiasmo, fantasie, } \\
\text { aspettative). Sguardo verso il }\end{array}$ \\
\hline 5. Partenza (lutto). & futuro. \\
\hline & 4. Partenza (trionfo). \\
\hline 6. Arrivo (aspettative). & 5. Viaggio. \\
\hline 7. Entusiasmo ed euforia. & 6. Arrivo (confusione). \\
\hline 8. Difficoltà, conflitti, rischi. & 7. Confusione e delusione. \\
\hline $\begin{array}{l}\text { 9. Cambiamenti e trasformazioni } \\
\text { personali. }\end{array}$ & $\begin{array}{l}\text { 8. Reazione - si inizia a lottare. } \\
\text { 9. Sistemazione logistica e lavorativa. }\end{array}$ \\
\hline 10. Ritorno (evocativo, immaginario). & 10. Ritorno (concreto). \\
\hline
\end{tabular}

Fonte: http://www.magma.analisiqualitativa.com/๑102/article_@6.htm\#1

\subsection{Aspetti psicologici legati all'acquisizione della L2}

Nella società europea, che si può definire sempre più multietnica e plurilingue, si sente l'esigenza di un'acquisizione di competenze linguistiche che possano agevolare e facilitare gli scambi comunicativi fra individui, da sempre in movimento, attori di spostamenti e cambiamenti di vita radicali, e che quindi hanno identità diverse fra loro. Volendo delineare un quadro degli elementi che influenzano e caratterizzano il contesto migratorio europeo e più nello specifico italiano, si forniscono all'interno di questo paragrafo delle riflessioni sugli aspetti psicologici e didattici che definiscono i percorsi di apprendimento della L2, sottolineando la necessità e il dovere di una formazione linguistica che favorisca l'integrazione sociale di ogni tipologia di individuo per un dialogo tra popoli e culture differenti.

All'interno del contesto migratorio è di primaria importanza la formazione linguistica, che ha il compito di determinare un livello di in- 
terlingua necessario per una buona integrazione sociale nel tessuto locale, talvolta però questo presupposto può provocare un disagio psicologico nelle persone migranti una volta arrivate nel Paese di accoglienza: è questo il caso di categorie di migranti vulnerabili come donne e bambini, e di fattori inerenti un individuo che entrano in gioco ed influenzano determinati processi come l'aspetto motivazionale e il disagio linguistico, ovvero quegli elementi che provocano stati di ansia all'insorgere di difficoltà che si possono incontrare nei percorsi di formazione linguistico-culturale, oltre alla paura del fallimento.

L'Italia, culla di varie civiltà del passato, possiede una lunga tradizione nell'accogliere popoli diversi e apprendenti di lingua italiana. Il boom economico degli anni Sessanta favorì l'aumento dell'immigrazione nel Paese e dalla seconda metà degli anni Ottanta le crescenti ondate migratorie incrementarono ulteriormente la domanda di competenza linguistica dell'italiano all'interno dei confini nazionali e di conseguenza la crescita di corsi specializzati. I primi corsi di lingua e cultura per stranieri furono istituiti a Siena nel 1917. Le istituzioni hanno posto attenzione a queste esigenze e hanno tentato di rispondere tempestivamente alla richiesta di istruzione dei cittadini stranieri entrati nel sistema educativo italiano creando un programma di valutazione del livello soglia per l'italiano capace di garantire lo sviluppo delle competenze necessarie la sopravvivenza nel contatto con i parlanti nativi, livello B1 del Quadro Comune Europeo di Riferimento.

Per avere un'idea più generale del fenomeno della migrazione oggi può risultare utile inquadrare la situazione a livello europeo. Difatti, le ondate migratorie di questi ultimi anni verso l'occidente stanno creando una nuova e interessante realtà.

Conseguentemente alla crescente presenza di figure femminili nei processi migratori a livello globale (cf. Decimo 2005) si è assistito, in Italia in particolar modo, ad un costante aumento delle donne sul totale dei cittadini stranieri: secondo l'ultimo Rapporto Immigrazione (Caritas, Migrantes 2020) si è vista una leggera prevalenza di donne straniere residenti in Italia, che costituiscono una media nazionale del 51,8\% con numeri molto più elevati nel caso di alcune nazionalità già storicamente caratterizzate da flussi migratori al femminile, come l'Ucraina (77,5\%), la Bielorussia, la Georgia e la Federazione Russa (tutti casi in cui le donne superano l'80\%). Frutto di questo notevole aumento è stato il sistema del ricongiungimento familiare, a partire dalla metà degli anni Novanta del secolo scorso, fenomeno che ha visto il suo ampliamento tra il 1993 e il 2001 e che ha visto in Italia un aumento esponenziale di ricongiungimenti familiari con l'entrata in vigore della Legge 40/49 che riconosce e tutela il diritto dei migranti a mantenere e/o a riacquisire l'unità familiare (cf. Cognigni 2014).

I bisogni linguistici e formativi delle donne migranti si presentano in un primo momento simili a quelli dei migranti uomini, soprat- 
tutto nella prima fase di assestamento nel territorio di accoglienza, il primo contatto diretto con la lingua e la cultura del Paese crea difficoltà o disagio anche se di natura differente in base al genere. Una prima differenza si può notare se ci si sofferma ad analizzare il background migratorio delle donne, che seguono rotte migratorie differenti, e al percorso di vita che intendono intraprendere nel nuovo Paese. Esse infatti necessitano di una maggiore attenzione sul piano della ricerca e delle pratiche didattiche che dovranno essere specifiche per questa tipologia di apprendente.

Appartenendo, come già detto precedentemente, ad una categoria svantaggiata e vulnerabile, data dalla loro condizione di migranti e dalla loro identità di genere, e sovente anche al non pieno riconoscimento dei loro diritti, dallo studio della letteratura di riferimento (Quercioli 2004; Favaro 2006; Solcia 2011; Cognigni 2013) si evidenziano alcune peculiarità rispetto ai loro bisogni linguistici e formativi:

- il bisogno di sviluppare con particolare attenzione le abilità di produzione e di interazione orale, a differenza degli uomini che hanno più opportunità di scambi comunicativi nella società di arrivo e che quindi possono acquisire spontaneamente i concetti di base della L2 (cf. Favaro 2006);

- il bisogno di capire i codici culturali di base e quelli legati alle specifiche pratiche di genere del contesto di accoglienza e di sviluppare una competenza d'uso della L2 per mantenere la funzione genitoriale nel Paese di accoglienza (cf. Quercioli 2004);

- il bisogno di acquisire un linguaggio dell'affettività che permetta loro di esprimere propri sentimenti ed emozioni, oltre allo sviluppo di una competenza linguistico-comunicativa di base per rispondere alle necessità strumentali (cf. Favaro 2006).

Data quindi la sempre più forte componente femminile fra le donne migranti in Europa e più nello specifico in Italia da una ricognizione sui corsi di italiano L2 è emerso anche un crescente numero di corsi dedicati a donne migranti, sui bisogni linguistici e formativi delle donne e a quelle che la sociologia della migrazione chiama «donne del ricongiungimento» (cf. Tognetti Bordogna 2002).

A partire dagli anni Novanta si sono diffusi in Italia i primi corsi di italiano L2 per donne migranti, sostenuti da enti locali ed associazioni di volontariato, che sono serviti da modello per poi oggi offrirne in tutta la penisola. Dalle prime esperienze formative hanno preso ispirazione diversi manuali di L2 specifici per questa categoria: Anche le mamme a scuola (Centro Come 2001), La storia di Naima (Favaro, Papa 1997), L'italiano con Naima (Veneri 2004).

La maggioranza delle donne che frequentano questi corsi sono ricongiunte, madri o neo-madri di origine spesso arabofona o donne che intraprendono il viaggio migratorio da sole provenienti dall'Est europeo che poi si impegnano nei servizi di cura della persona. 
La pianificazione di questi specifici corsi è motivata dall'invisibilità sociale di questa categoria di apprendenti (cf. Favaro 2006), a causa della limitata indipendenza sociale e lavorativa, dalla complicazione nel frequentare corsi di 'prima alfabetizzazione' se misti e dalle ridotte occasioni di interazione con autoctoni a causa della provenienza culturale o familiare. Molto spesso queste donne si presentano ai corsi di formazione dopo lunghi periodi di permanenza sul territorio ospite e quindi hanno sviluppato in modo spontaneo conoscenze di base della L2 grazie ai contatti con gli abitanti, con i connazionali o con l'uso della televisione.

La realizzazione di questi corsi al femminile è dunque necessaria per offrire opportunità migliori nell'ambito del ricongiungimento alle donne che se non avessero possibilità di scelta rimarrebbero un 'pubblico potenziale' come suggerito da Vedovelli (2010).

L'offerta formativa generalmente si basa su un approccio comunicativo-affettivo che pone al centro l'acquisizione orale e sul campo, facilitando così anche le donne con bassa scolarizzazione. I sillabi di riferimento su cui vengono costruiti i percorsi di formazione sono attenti ai bisogni di genere e, come nel caso del manuale per donne migranti di Marelli e Rodondi (2013), all'educazione alla cittadinanza, oltre ad offrire strumenti linguistico-comunicativi utili al raggiungimento del livello A1 (di contatto) e A2 (di sopravvivenza) e permettono alle apprendenti di acquisire competenze utili a:

- facilitare l'accesso ai servizi e l'inserimento nel contesto socioculturale italiano;

- ottenere un'autonomia linguistica rispetto alla figura maschile che hanno vicino;

- poter essere coinvolte nel percorso scolastico dei figli per migliorare la condizione del rapporto scuola-famiglia.

La tipologia di programmazione e la scelta dei materiali didattici generalmente affrontano tematiche specifiche: la famiglia, la scuola, i servizi sanitari ecc. Tutte questioni legate alla quotidianità delle donne migranti, in quanto madri e mogli, che mirano ad aumentare la componente motivazionale dando una visione di utilità nel soddisfare i loro bisogni linguistico-comunicativi (ad esempio fare la spesa, comprare dei medicinali, saper interagire con i dottori e gli insegnanti ecc.), ma allo stesso tempo rischia di subire uno sbilanciamento e una forzatura confinando le donne migranti a specifici domini comunicativi.

Rispettando la loro condizione familiare e sociale nella formazione linguistico-culturale si cerca di rispondere ai bisogni che concernono la sfera dell'affettività e della socialità, come suggerito da Favaro (1996):

- il «bisogno di trovare un clima di accoglienza e ascolto per apprendere ed inserirsi meglio nella nostra cultura»; 
- il bisogno di «creare momenti di socializzazione tra le iscritte, che oltre ad essere aggregativi servano anche a creare reti sociali utili (scambio di informazioni, mutuo aiuto...)»;

- il bisogno di autonomia e di espressione di sé stesse: «lo studio della lingua italiana per la donna [migrante] è motivo per uscire di casa, dalla routine. Si prende uno spazio tutto suo molto importante».

I corsi di formazione di L2 sono quindi un'opportunità

per raccontarsi alle altre, dire qualcosa di sé e della propria storia [...] l'occasione anche per manifestare il disagio condiviso per l'insopportabile silenzio che la migrazione porta con sé, per lo meno in una prima fase» (Favaro 1996, 117),

adottando quindi un approccio autobiografico in quanto

«parte del vissuto quotidiano per riandare alla storia passata e spingersi poi verso i progetti e i sogni del futuro, per se e per i figli [...] si apprendono così per parole e le strutture della nuova lingua che servono per dire il presente, raccontare il passato, esprimere sogni, possibilità e punti di vista. (Favaro 2006, 5)

Dunque l'apprendimento della L2 per le donne migranti deve poter essere un veicolo per una educazione alla cittadinanza che fornisca gli strumenti linguistico-comunicativi idonei all'acquisizione di un'autonomia interazionale, espliciti i modelli culturali del contesto migratorio in relazione all'accesso dei servizi sociosanitari e scolastici, mettendole in condizione di poter conoscere i propri diritti di donna e di madre nel contesto di arrivo. Una formazione che in qualche modo tenga in considerazione i loro progetti e le aspirazioni future e il loro retroterra culturale e linguistico.

L'importanza dell'integrazione della lingua degli immigrati all'interno del sistema educativo italiano è stata più volte sottolineata dai provvedimenti legislativi dell'Unione Europea e dagli atti di indirizzo del Consiglio d'Europa. Vedovelli ha infatti sottolineato, unitamente a un'esortazione al riconoscimento e all'attenzione riguardo gli atteggiamenti linguistici che allontanano il parlante italiano da quello immigrato, l'importanza delle lingue nei progetti migratori:

Per quanto riguarda gli immigrati, la lingua esalta la sua funzione di principio di identità innescando processi su varie dimensioni: nella lingua si catalizzano i processi di identità perduta (quando si lascia il proprio Paese e si perde il contatto quotidiano con la propria lingua), cercata (nel tentativo di essere nel Paese ospite, di esistere secondo una qualche identità capace di dare sen- 
so), scissa (quando le due identità culturali non si ricompongono e l'apprendimento scolastico si blocca e non consente l'integrazione), equilibrata (quando il migrante segnala nella adeguata competenza linguistica il successo migratorio che non rinnega le radici d'origine). In tutti questi processi, che riguardano sia gli adulti che i bambini, la lingua e strumento dei processi di identità e segnale dello stato del processo. (Vedovelli 2005, 35)

A differenza di quanto accade per l'italiano all'estero, in Italia l'apprendente tipico di italiano L2 che non rientri nelle categorie degli adulti o minori immigrati è un adulto, più spesso un giovane adulto, nella fascia di età tra i 18 e 30 anni cioè in quel periodo della vita in cui si svolgono gli studi fondamentali per la propria futura esperienza professionale umana, si accumulano titoli e certificazioni, si inseguono interessi, passioni e persone significative della propria crescita personale, si compiono viaggi formativi nel proprio Paese, si fanno esperienze di tirocinio o lavoro temporaneo, si cercano occupazioni che garantiscano una nuova possibilità di integrazione sociale e benessere economico. Al di là della categoria di appartenenza l'apprendente tipo di italiano L2 è definito sulla base della sua permanenza in Italia per un periodo più o meno lungo durante il quale si troverà a frequentare un corso di italiano per stranieri.

È ovvio che l'attenzione che le istituzioni italiane dovrebbero dare a queste lingue, si dovrebbe concretizzare in azioni, fra le quali è centrale lo studio a scuola della lingua madre degli immigrati. A tal proposito riprendendo ancora una volta le parole di Vedovelli, bisogna ricordare che «tra i contesti istituzionali la scuola è un luogo centrale per produrre sistemi di scambio sociale e comunicativo che favoriscono lo sviluppo della competenza linguistica degli immigrati adulti e bambini» (Vedovelli 2005, 40).

Lo studio della lingua italiana è dimostrato diffuso in tutto il mondo, con alterne vicende di gradimento nei vari periodi a partire dalla metà degli anni Ottanta del secolo scorso, con motivazioni differenti e coinvolge varietà di pubblici distinti. Alla tradizionale categoria di coloro che si trovano ad apprendere la lingua fuori dall'Italia si è andata via via sovrapponendo e in parte sostituendo quella di coloro che scelgono di studiarla nel territorio italiano sia per motivi latamente culturali che per necessità. Concentrandosi sullo studio della lingua all'interno dei confini del Paese si possono dunque individuare due tipi di beneficiari: gli immigrati in Italia per motivi di necessità, che devono inserirsi nel contesto sociale e lavorativo come gli adulti e nel contesto scolastico come bambini e adolescenti, e chi sceglie di soggiornare o vivere nel Paese perché ne apprezza lo stile di vita, la cultura, il clima (cf. Benucci 2018). Per queste due tipologie di apprendenti si manifestano bisogni di natura differente. Nel primo caso è richiesto un apprendimento universale, che quindi andrà 
a rispondere a quei criteri obiettivi stabiliti dalle istituzioni e richiesti per l'inserimento nella società. Nel secondo caso invece, si parla di apprendimento specifico per il singolo individuo in quanto si trova a studiare la lingua e cultura italiana per un tempo determinato, sia per studio che per vacanza, e con obiettivi intrinsechi ai suoi interessi nei confronti dell'Italia e degli italiani. I bisogni linguisticoculturali, nella loro totalità e sfumature, sono correlati alla stesura di sillabi che nel loro essere si occupano di pianificare il lavoro e gli obiettivi da raggiungere, «il concetto di bisogno pone l'attenzione sui motivi per i quali si apprende una lingua e per raggiungere quali fini, quindi è fondamentale per impostare l'azione di programmazione didattica» (Benucci 2018, 40). Come suggerito sempre da Benucci (2018), sulla base della natura dei bisogni e della motivazione, è possibile definire gli obiettivi che si suddividono in: comunicativi, sociali, psicologici, di studio, politici ed economici, generali ecc. Questi a loro volta stabiliscono differenti tipologie di sillabo. Per esempio, per il profilo dell'immigrato di interesse in questo capitolo la necessità è quella di andare ad organizzare e lavorare sugli aspetti linguistico-comunicativi del linguaggio burocratico, sia orale che scritto.

In ogni caso, ogni qualvolta sarà possibile, si partirà dal contatto delle lingue e culture coinvolte per domandarsi quali caratteristiche specifiche della lingua degli studenti possano essere utili ai fini dell'apprendimento dell'italiano [...], nelle situazioni di diglossia quali sono le varietà di maggiore prestigio e quali sono i modelli culturali ad esse associati; su quali abilità occorrerà insistere per studenti universitari [...], quali elementi di cultura possano creare disagio o errata interpretazione negli adulti immigrati, quali tipologie di testi saranno più utili. (Benucci 2018, 41)

Secondo Vedovelli gli immigrati vivono in realtà complesse, il loro repertorio linguistico viene per questo stimolato in tutte le sue parti. La figura dell'immigrato adulto si trova a dover agire in situazioni di vita e che richiedono competenze differenti, con la necessità di gestire il verificarsi di un contatto linguistico su più livelli. La loro L2 dovrebbe comprendere le varietà substandard dell'italiano, talvolta anche dei dialetti, parlati nel territorio in cui vive per poter avere scambi comunicativi nelle situazioni informali; dovrebbero altresì saper utilizzare distinguendoli nei vari usi gli elementi del registro formale nei contesti adeguati. Dovrebbero infine poter comprendere indicazioni complesse, che spesso si rivelano di vitale importanza sia in termini di svolgimento del lavoro che di sicurezza personale (cf. Vedovelli 2002). Nonostante la complessità dei compiti richiesti, spesso sia l'input linguistico rivolti agli immigrati che l'output non sono sufficienti. 
Dal punto di vista psicologico si crea uno stato di anomia nella fase di integrazione unito alla vergogna della inferiorità linguistica dovuta alla mancanza del possesso pieno del repertorio necessario per vita di tutti i giorni. A ciò generalmente si aggiungono fattori demografici e socioculturali come l'età non più giovane, il matrimonio contratto tra connazionali, il tipo di mansioni svolte sul lavoro e povero di comunicazione: tutto ciò porta ad uno scarso apprendimento della nuova lingua. (Benucci 2018, 44)

Rispetto a quanto detto da Benucci, le ricerche sulla produzione linguistica di studenti di diverse nazionalità dimostrano che nell'apprendimento della lingua ospite gli errori sono simili e quindi non derivano sempre da interferenza dei due sistemi linguistici in contatto, ma sono errori di tipo evolutivo e ciò significa che il processo di acquisizione del linguaggio è uguale per tutti in modo creativo. Gli studenti stabilizzano e uniscono le funzioni delle regole quelle strutture della L2 guidati da un meccanismo universale innato in cui il concetto di transfert assume un valore più ampio rispetto all'incrocio della L1 con la L2, ma comprende anche le proprie ipotesi e regole create dall'apprendente sulla propria L2 (cf. Benucci 2018, 42).

Secondo Ellis (2005) non ci sono evidenti differenze nella formazione delle interlingue in base all'età, alla lingua materna, i percorsi sembrano esseri simili. È chiaro che l'apprendente adulto ha tempistiche e modalità di acquisizione ben diversi da quelli di un bambino o di un adolescente. Un adulto con una forte motivazione apprende più velocemente di un bambino perché sa individuare la finalità del lavoro che sta svolgendo, ma anche per la sua competenza cognitiva e metalinguistica più sviluppata e una coscienza della realtà del mondo che lo circonda che gli permette di ricorrere ad ogni 'sapere' già posseduto (cf. Krashen, Long, Scarcella 1982).

Dalla seconda metà degli anni Ottanta del secolo scorso la lingua italiana ha visto un suo sviluppo anche all'interno dei confini nazionali grazie all'approdo di comunità straniere che richiedevano di acquisire competenze linguistico-comunicative sufficienti a vivere in Italia e quindi a compiere e a portare a termine il loro piano di migrazione. Nonostante l'incombenza della crisi economica, la linea di crescita migratoria non ha visto un'involuzione, bensì ha modificato e promosso la propria domanda di formazione linguistica rivolgendosi ad università, scuole di ogni grado e genere, associazioni ed enti territoriali.

La popolazione straniera presente in Italia ha continuato a crescere subendo un incremento consistente rispetto agli anni precedenti, rilevando altresì dati numerici in aumento relativi alla stabilità territoriale, quindi alla richiesta di residenza. Se fino a un decennio fa l'aumento della popolazione straniera seguiva un ritmo significativo, da qualche anno il trend è in diminuzione (dal 2018 al 2019 appe- 
na 47 mila residenti e 2.500 titolari di permesso di soggiorno in più), accompagnato da altri segnali 'negativi', come la diminuzione delle nascite (da 67.933 nel 2017 a 62.944 nel 2019) e le minori acquisizioni di cittadinanza (passate da 146 mila nel 2017 a 127 mila del 2019) (cf. Caritas, Migrantes 2020). Con questa stabilizzazione permanente, le comunità migranti hanno evoluto la propria struttura familiare, incrementando la presenza di adolescenti e bambini bisognosi di formazione linguistica per l'accesso alla scuola in quanto obbligatoria, nel 2013 infatti, secondo i dati ISTAT, risultavano un milione cittadini stranieri tra zero e 18 anni residenti in Italia. ${ }^{2}$

Come evidenziato da Diadori, Palermo e Troncarelli (2021), molti di questi bambini crescono apprendendo due lingue in quanto figli di un genitore migrante e uno nativo del luogo di residenza. Altri invece, dopo aver appreso la lingua della famiglia entrano in contatto fin da subito con l'italiano avendo scambi comunicativi con fratelli maggiori, compagni di scuola ecc. Infine, si presenta il caso dei bambini/ adolescenti che apprendono la lingua italiana solo dopo il loro inserimento nella scuola italiana.

Per descrivere la complessità e la peculiarità di questo contesto di insegnamento-apprendimento risulta riduttivo ricorre all'espressione italiano lingua seconda (L2), che non consente di cogliere l'articolata situazione linguistica in cui si trovano i minori migranti [...] Si è preferito quindi riferirsi all'italiano insegnato e appreso dai figli di cittadini immigrati in Italia con la denominazione 'italiano lingua di contatto', con la quale si mira a porre in evidenza la natura composita della competenza individuale di questa tipologia di apprendenti, che comprende l'italiano e la lingua d'origine, producendo attraverso il contatto sollecitazioni relative alla definizione della propria identità linguistica e culturale. (Diadori, Palermo, Troncarelli 2021, 94)

Espressione introdotta da Weinreich (1974) negli studi di sociolinguistica, da Freddi (1987) in glottodidattica, da Tullio de Mauro nei documenti di sintesi dei lavori di gruppo della Commissione di studio per il programma di riordino dei cicli di istruzione e infine da Vedovelli (2002a) che da spazio all'espressione utilizzata in glottodidattica e che successivamente chiarisce:

Per i giovanissimi cittadini italiani di famiglia straniera o mista (e non stranieri, come sono spesso chiamati), l'italiano non è spesso nettamente né lingua madre, cioè lingua dell'identità primaria, né lingua straniera o seconda, cioè oggetto di una sovrappo- 
sizione acquisizionale successiva al processo di primario sviluppo della competenza linguistica. Per le giovani generazioni di origine straniera l'italiano entra nella coscienza e nell'identità linguistica a costruire un continuum con altri idiomi: la lingua dell'ambiente familiare, i dialetti. L'italiano, allora, contribuisce a creare un ambiente di contatto, dove l'individuo costruisce e ricostruisce la propria identità innanzitutto linguistica: solo la scuola e una società ancora troppo cieca, sorda e muta nei confronti della diversità linguistica possono non comprendere i fenomeni che si stanno producendo. L'italiano non può essere visto come una lingua straniera, nettamente distinta dalla 'lingua madre': l'italiano crea un territorio di confine e di contatto che rende possibili intricate sovrapposizioni, scambi, interferenze che costituiscono le risorse espressive e, soprattutto, di identità dei soggetti. In tale visione il contatto, l'interferenza, il miscuglio sono visti come elementi positivi, fonti di ricchezza identitaria ed espressivo-comunicativa. (Vedovelli 2005b, 27-8)

Come anche dichiarato da Diadori, Palermo e Troncarelli (2021), la posizione di Vedovelli permette di cogliere l'influenza dei fattori psicoaffettivi, identitari e socioculturali nel processo di apprendimento dell'italiano, per poi progettare azioni didattiche focalizzate sulla centralità dell'apprendente, metodologie e strategie di insegnamento per lo sviluppo di competenze linguistico-comunicative per l'integrazione, grazie alla connessione nel momento di contatto della storia linguistica e culturale dei bambini e adolescenti con la lingua e cultura italiana.

Nel percorso di apprendimento linguistico di un individuo sono altrettanto importanti le motivazioni e le scelte legate alla socializzazione linguistica, che entrano in gioco sul piano psicologico.

La motivazione, pur sembrando un concetto semplice, appare essere un discorso complesso se se ne cerca il significato in psicologia e si tenta di capire come essa funzioni: è un processo che si osserva attraverso i comportamenti messi in atto da un individuo per realizzare determinati obiettivi con specifici motivi. Grazie allo schema ripreso da Moè $(2010,22)$, è possibile comprendere che la motivazione scaturisce se si è all'interno di una situazione che aiuta a concretizzarla, altrimenti non nasce dal nulla all'interno di ogni individuo, in quanto nessuno è motivato e nessuna situazione è motivante di per sé.

Ciascun tipo di motivazione è composta da due elementi: l'avvicinamento e l'allontanamento. Il primo induce ad affrontare una situazione e produce di conseguenza un comportamento consono; il secondo conduce al comportamento contrario e quindi a rifiutare di cimentarsi in una situazione. Entrando nello specifico, è possibile dire che la motivazione è costituita da una serie di esperienze personali: a partire da esse si arriva ad un dato comportamento, ad un obiet- 
tivo o ad un atteggiamento (cf. De Beni, Moè 2000). Naturalmente, le motivazioni sono di diversa natura: le attività svolte perché legate ad un obbligo o ad una percezione negativa di ciò che si sta facendo, visto come poco utile o sbagliato, producono uno stato di insofferenza e quindi emozioni negative; invece, le attività svolte con presupposti positivi e quindi mosse da un'esplicita volontà dell'individuo, producono stati emotivi positivi e una durata nel tempo maggiore. Bisogna pertanto tenere conto del fatto che esiste un importante legame tra motivazione ed emozione: tra questi due elementi nella maggior parte dei casi si instaura una relazione descrivibile in modo circolare, come se si autoalimentassero costantemente durante il processo di costituzione della motivazione complessiva. La motivazione non è qualcosa di statico e immutabile, anche nel caso in cui inizialmente sia negativa è comunque soggetta a modifiche: spesso influenzate dal contesto, dalla situazione familiare o da chi è responsabile della formazione linguistico-culturale, sollecitate quindi da fattori circostanti; in alcuni casi però le motivazioni sono autogenerate. Certi processi possono concorrere a creare una motivazione: nel caso in cui si assista ad un'azione combinata di questi processi e questi a loro volta puntino nella stessa direzione, allora si avrà come risultato il prodursi di un comportamento volto a realizzare l'attività accompagnata da emozioni positive; in altri casi però, l'individuo può essere coinvolto in una sorta di conflitto in cui sussistono contemporaneamente forze pro e contro una determinata azione, che puntano da una parte alla realizzazione dell'attività e dall'altra alla non realizzazione della stessa (approach / avoidance). Come suggerito da uno studio di Pintrich (2003) le motivazioni di tipo cognitivo sono meno durature e stabili ma si tratta di un elemento relativo, infatti è possibile agire sul proprio sistema motivazionale al fine di indirizzarlo verso un obiettivo. L'essere umano è geneticamente portato a mantenere una stabilità e alla conservazione delle proprie caratteristiche, positive o negative che siano, perciò non è facile che un riordino delle motivazioni intervenga e origini un cambiamento, soprattutto se questo può andare a sollecitare emozioni che l'inconscio non lascia libere di esplicitarsi. Infine, occorre dire che le motivazioni sono di tipo primario e secondario: le prime sono comuni ad esseri umani e animali e appartengono alla sfera dei bisogni primari, mentre le seconde sono esclusive dell'ambito umano e si rifanno ad uno scopo preciso, quello della realizzazione personale, allo sviluppo delle proprie potenzialità o allo sviluppo di determinati valori; entrambe sono presenti nell'individuo e agiscono simultaneamente le une sulle altre. Bisogna considerare, inoltre, il fattore dell'accessibilità, infatti esistono motivazioni esplicite accessibili e definibili come immagini del sé motivazionale che si contrappongono alle motivazioni implicite, le quali sono generalmente meno accessibili a seconda di come l'inconscio agisce su di esse. Secondo le indagini svolte da McClelleland, Ko- 
estner e Weinberger (1989), le motivazioni implicite risultano meno accessibili delle altre in quanto pertengono al livello dell'inconscio, sono però più stabili rispetto a quelle esplicite e di conseguenza più conoscibili ma meno individuabili se non con appositi test. È dimostrato che i due livelli coesistono simultaneamente e possono quindi agire nello stesso tempo ma in maniera differente, generando così difficoltà sull'efficacia dell'intervento svolto per il raggiungimento di un determinato obiettivo.

Talvolta però la motivazione non è l'unico problema che ostacola l'apprendimento di una nuova lingua: nei percorsi di formazione linguistico-culturale si possono generare problematiche linguistiche di tipo psicologico causate da quelle che si definiscono situazioni di svantaggio linguistico. Come spiegato da studi di psicolinguistica (Marini 2001; Steinberg, Sciarini 2006) una situazione di svantaggio linguistico si determina quando si verifica un disagio per il quale si costituisce un ostacolo che coinvolge lo sviluppo cognitivo, relazionale e sociale dell'apprendente: il disagio linguistico genera un debole funzionamento adattivo che può contribuire, nei peggiori dei casi, ad un degradamento della sua immagine personale. Il discente che sperimenta una situazione di svantaggio linguistico o culturale, può vivere un'esperienza di fragilità emotiva e psicologica, la quale influenza le proprie relazioni con l'ambiente circostante, con i contesti sociali o lavorativi e con le persone con le quali interagisce. Per queste problematiche si può intervenire in diversi modi: innanzitutto è necessario individuare lo stato di disagio emotivo per poter agire in termini di riparazione, anche psicologica ma soprattutto occorre determinare lo svantaggio in base ad elementi oggettivi, per esempio capendo cosa caratterizzi la difficoltà ad apprendere le Basic Interpersonal Communicative Skills; ${ }^{3}$ in secondo luogo è opportuno effettuare considerazioni di tipo psicopedagogico e didattico, in seguito ad un'attenta osservazione realizzata da colui che intuisce questo tipo di disagio, l'insegnante, il quale parte dalle valutazioni negative riportate nel tempo sia nell'ambito didattico che in quello relazionale. Il docente è la figura più idonea per osservare in modo sistematico le esigenze degli apprendenti, cercando di stabilire quali possano essere i bisogni peculiari di persone che manifestano questo particolare stato. Per questi si può quindi predisporre un Piano Didattico Personalizzato affinché si possano affrontare gli specifici bisogni di chiunque ne soffra: un Piano Didattico di questo tipo deve prevedere misure di tipo dispensativo, criteri e strategie di intervento, adatti a superare le difficoltà manifestate attraverso l'adozione di strumenti compensativi. Queste misure dispensative saranno di carattere transitorio, atte a modificare nel breve perio- 
do la situazione di disagio, adottando strategie linguistiche e didattiche che abbiano come obiettivo il superamento degli ostacoli presentati. Un Piano Didattico Personalizzato può e deve essere varato per chi presenta uno svantaggio linguistico e culturale, ed è quindi rivolto a tutti gli apprendenti stranieri appena arrivati in terra straniera che non hanno ancora acquisito adeguate competenze per instaurare relazioni interpersonali, predisponendo attività di supporto allo studio e percorsi linguistici di L2. È assolutamente indispensabile anche per tutti coloro che manifestano un disagio relazionale 0 psicologico: si parla di apprendenti che esternano un relazionamento problematico a qualsiasi piano che determina difficoltà ad interagire con l'ambiente circostante o che esibiscono una particolare fragilità emotiva/psicologica. La psicolinguistica entra a pieno titolo in questo discorso: questa disciplina utilizza strumenti e metodi di vario tipo per la determinazione di casistiche come quella presentata. In Marini $(2001,18)$ si legge che il punto di partenza consiste nell'osservazione comportamentale del soggetto in cui si manifesta un disagio; tale azione preparatoria mira ad osservare i fenomeni e gli errori linguistici che vengono compiuti. Un'altra tecnica consiste nel misurare i tempi di reazione durante l'emissione di un enunciato, infatti l'indecisione circa il lessema da utilizzare o l'uso scorretto di una regola sintattica che teoricamente dovrebbe essere stata assimilata da tempo, sono spie che il docente può cogliere per capire se si trova davanti ad un caso di disagio linguistico particolare che richiede un'azione mirata per il suo recupero. 


\section{Problematiche e soluzioni per apprendenti analfabeti e per classi ad abilità differenziate}

Giulia I. Grosso

Università degli Studi di Cagliari, Italia

Sommario 5.1 Analfabetismo e migrazione. - 5.2 Bisogni delle classi ad abilità differenziate. - 5.3 Nuove tecnologie a supporto della lotta all'analfabetismo.

\subsection{Analfabetismo e migrazione}

In questo capitolo si delineerà un quadro teorico-pratico sul fenomeno dell'analfabetismo nelle società europee in relazione ai fenomeni migratori. Si individueranno i contesti all'interno dei quali l'analfabetismo si manifesta nelle sue molteplici accezioni e all'interno dei quali vengono proposte soluzioni per dotare gli apprendenti di competenze ormai fondamentali per vivere nella contemporaneità, ovvero i contesti formativi e il carcere; infine verrà descritto l'uso didattico delle nuove tecnologie e alcune buone pratiche esistenti in questa direzione, per concludere con una batteria di domande attraverso le quali i docenti possono orientarsi nella valutazione dei materiali didattici per apprendenti analfabeti o a bassa scolarizzazione.

Si ringrazia sentitamente Ida Ferrari, collega e amica, per l'attenta lettura di questo capitolo e per i preziosi suggerimenti forniti. 
Il possesso delle competenze in letto-scrittura, o literacy, può essere a pieno titolo annoverato tra i diritti umani fondamentali secondo l'articolo 26 sul diritto all'educazione nell'ambito della Dichiarazione Universale dei diritti umani, dal momento che il legame tra la mancanza di queste competenze e la possibilità di scivolare in processi di esclusione sociale e povertà è dimostrato.

Nel 2017, secondo i dati dell'UNESCO ${ }^{1}$ il tasso di alfabetizzazione globale era stimabile intorno all' $86 \%$, con circa 750 milioni di adulti in condizione di analfabetismo (a vario grado) e con una significativa percentuale di differenza tra aree geografiche e tra uomini e donne, in particolare nei Paesi in via di sviluppo. Sebbene la definizione di literacy non abbia confini netti, ma al contrario, come sostenuto dall'UNESCO, costituisca piuttosto un continuum che va dalla mancanza di competenze in letto-scrittura all'analfabetismo funzionale, è importante osservare come le politiche di inclusione dei Paesi europei, meta di flussi immigratori importanti e aumentati del $28 \%$ nell'ultima decade secondo i dati i dati OCSE, ${ }^{2}$ abbiano dovuto tenere in considerazione tale condizione o la sua eventuale assenza.

Gli adulti immigrati che sperimentano questa difficoltà, infatti, a diversi livelli, possono essere considerati soggetti vulnerabili, e questo avviene in particolare quando i programmi educativi basati sullo studio delle lingue dei Paesi di arrivo come prima tappa verso l'integrazione sociale non prevedono l'affiancamento di percorsi di alfabetizzazione.

La definizione di 'analfabeta', tuttavia, deve essere ulteriormente articolata e approfondita, al fine di comprendere a fondo i bisogni di cui ciascun gruppo specifico di apprendenti è portatore. Si tratta di persone che vivono e lavorano nelle società europee, che hanno retroterra eterogenei per lingua madre (spesso tipologicamente distante dalla lingua del Paese target), età, traiettorie migratorie, anni di scolarizzazione, ampiezza delle reti sociali in cui sono inseriti (caratterizzate da interazioni più o meno frequenti con altri individui nella società di arrivo), motivazioni più o meno forti all'apprendimento della L2. Come dimostra uno studio recente condotto in Germania (Scheible 2018), di cui si parlerà più avanti, però, gli analfabeti costituiscono proprio il gruppo target che, nonostante l'evidente necessità di percorsi formativi ad hoc, ha maggiori difficoltà ad essere inserito in corsi di lingua finalizzati all'integrazione sociale, dando vita a un 'circolo vizioso' che li candida ad essere marginalizzati ed esclusi socialmente. Tale situazione, osservata in molti contesti europei fra i quali l’Italia, è stata oggetto di denuncia da parte di do-

1 http://uis.unesco.org/sites/default/files/documents/fs45-literacy-ratescontinue-rise-generation-to-next-en-2017.pdf.

2 https://www.oecd-ilibrary.org/docserver/9789264307216-en.pdf. 
centi e istituzioni che quotidianamente si confrontano con le difficoltà affrontate dagli apprendenti analfabeti. ${ }^{3}$

Il 24 novembre 2020 la Commissione europea ha pubblicato il nuovo EU Action Plan on Integration and Inclusion (2021-27), all'interno del quale è presente una attenzione specifica nei confronti dell'education and training come primi elementi per un'integrazione di successo/efficace dei migranti nelle società europee.

Nel documento Global Monitoring Report 2006, Literacy for Life si evidenziano le difficoltà di inserimento di questo target all'interno dei corsi.

Gli Stati membri hanno dunque adottato una nuova Strategia UNESCO (2020-25) per l'alfabetizzazione dei giovani e degli adulti con quattro aree strategiche prioritarie:

- sostenere gli Stati membri nello sviluppo di politiche e strategie nazionali di alfabetizzazione;

- affrontare i bisogni di apprendimento dei gruppi svantaggiati, in particolare donne e ragazze;

- sfruttare le tecnologie digitali per espandere l'accesso e migliorare i risultati dell'apprendimento;

- monitorare i progressi e valutare le competenze e i programmi per l'alfabetizzazione.

I Paesi e le popolazioni che devono gestire le maggiori sfide nel mondo includono i 29 Paesi membri dell'Alleanza globale per l'alfabetizzazione nel quadro dell'apprendimento permanente (GAL) che è coordinato dall'Istituto UNESCO. L'Alleanza è composta da 20 Paesi con tassi di alfabetizzazione degli adulti inferiori al 50\% e dai Paesi E9, un consorzio dei 9 Paesi più popolosi che ospitano oltre la metà della popolazione mondiale, con l'obiettivo di condividere le migliori pratiche di promozione dell'alfabetizzazione in una prospettiva di apprendimento permanente.

La strategia, in linea con il Sustainable Goal on Education 4 (SDG4) e l'Agenda 2030 per l'istruzione, si concentra anche sull'uguaglianza di genere e affronta i bisogni di apprendimento delle popolazioni cosiddette 'vulnerabili', compresi i giovani che non frequentano la scuola, i rifugiati, i migranti e le popolazioni indigene che fanno parte dei 773 milioni di adulti in tutto il mondo che non hanno competenze di alfabetizzazione di base.

In tutti i Paesi coinvolti in fenomeni migratori caratterizzati dalla presenza di persone analfabete o a bassa scolarizzazione si è quindi determinata la necessità di ridisegnare la didattica della seconda lingua, adattandola alle esigenze di coloro che hanno necessità di

3 Si vedano ad esempio il documento Il diritto al codice rosso per gli analfabeti: le anomalie italiane nell'istruzione degli adulti, e l'appello Analfabetismo: paralisi e cura per l'Italia (https://www.change.org/p/analfabetismo-paralisi-e-cura-per-l-italia), promossi dalla prof.ssa Paola Casi con Tullio De Mauro come primo firmatario. 
sviluppare maggiori competenze in letto-scrittura. Il mondo dell'educazione e della didattica ha risposto a questa richiesta da una parte generando nuovi modelli e tecniche appropriati per questo profilo di apprendenti, radicalmente diversi da quanto sperimentato in precedenza, come l'approccio whole language, il Language Experience Approach, e la pedagogia Freireana (Wrigley, Guth 1992, 10), o, dall'altra, adattando modelli didattici preesistenti.

Le competenze di letto-scrittura degli individui per le quali si è resa necessaria la nascita di nuovi modelli o l'adattamento dei preesistenti sono però diversificate e si collegano a un aspetto preliminare che è necessario adottare come premessa: la definizione di cosa si intende per 'alfabetizzazione' e 'analfabeta'.

Come sosteneva Tullio De Mauro, ${ }^{4}$ non è sufficiente infatti analizzare l'analfabetismo e definirne le caratteristiche, è necessario comprendere la distribuzione delle conoscenze attestate dal titolo di studio, nonché la funzionalità delle conoscenze possedute.

Sulla scorta di questa considerazione, si può affermare che la definizione del termine 'alfabetizzazione' in sé è particolarmente complessa: la sua concezione in termini di pratica sociale abbraccia una serie di competenze che vanno dalle competenze di letto-scrittura alle competenze numeriche e digitali, spingendo l'evoluzione del dibattito verso l'uso del termine ombrello 'alfabetizzazioni', che dovrebbe quindi comprendere queste diverse competenze e molte altre, come ad esempio le competenze digitali ormai considerate imprescindibili.

Secondo una definizione fornita dall'UNESCO nel 2005, la literacy si identifica quindi con «l'insieme di competenze che utilizzano le capacità di identificare, comprendere, interpretare, creare, comunicare e computare utilizzando materiale scritto derivante da vari contesti». Inoltre, «la literacy identifica un apprendimento continuo negli individui quando tendono ai loro traguardi, allo sviluppo della loro conoscenza e delle loro potenzialità e alla piena partecipazione alla vita delle comunità e delle società» (UNESCO 2005). ${ }^{5}$ La complessità di questa nozione, infatti, ha portato molti studiosi a non definirne nettamente i confini quanto piuttosto a considerarla da una parte come il risultato di una pluralità di literacies in diversi campi (alfabetizzazione sanitaria, alfabetizzazione numerica ecc.) e dall'altra come un continuum (Minuz 2019; Borri et al. 2014) di scale di competenza, indicate anche dal Consiglio d'Europa come literacy profiles, concetto che verrà ripreso più avanti.

\footnotetext{
4 http://nuovoeutile.it/istruzione-tullio-de-mauro-se-un-mattino-di-primavera-un-governante.

5 Traduzione presente in: https://www.treccani.it/magazine/lingua_italiana/ articoli/scritto_e_parlato/literacy.html.
} 
Per richiamare una visione multisfaccettata del concetto di literacy, che non si limiti a descrivere la stessa nel quadro del possesso delle competenze di letto-scrittura, è possibile anche fare riferimento alla definizione che di esso viene fornita all'interno del Rapporto nazionale sulle Competenze degli Adulti (ISFOL 2014, 24),

l'interesse, l'attitudine e l'abilità degli individui ad utilizzare in modo appropriato gli strumenti socio-culturali, tra cui la tecnologia digitale e gli strumenti di comunicazione per accedere a, gestire, integrare e valutare informazioni, costruire nuove conoscenze e comunicare con gli altri, al fine di partecipare più efficacemente alla vita sociale.

Nel report relativo all'indagine Adult Literacy and Life skills (ALL) - Competenze della popolazione adulta e abilità per la vita (Gallina 2005), la literacy viene rappresentata come insieme di competenze:

1. competenza di prose e document literacy: competenza alfabetica funzionale relativa alla comprensione di testi in prosa e formati quali grafici e tabelle; capacità di utilizzare testi stampati e scritti necessari per interagire con efficacia nei contesti sociali di riferimento, raggiungere i propri obiettivi, migliorare le proprie conoscenze ed accrescere le proprie potenzialità;

2. competenza di numeracy: competenza matematica funzionale; capacità di utilizzare in modo efficace strumenti matematici nei diversi contesti in cui se ne richiede l'applicazione (rappresentazioni dirette, simboli, formule, che modellizzano relazioni tra grandezze o variabili);

3. competenza di problem solving: capacità di analisi e soluzione di problemi; il problem solving rileva l'attività ragionativa in azione: pensiero orientato al raggiungimento di uno scopo in una situazione in cui non esiste una procedura di soluzione precostituita.

Insieme alle competenze di numeracy e di problem solving, la literacy così definita fa parte delle cosiddette information processing skills, le 'competenze chiave' considerate necessarie a utilizzare saperi, abilità e conoscenze per poter vivere e lavorare nel XXI secolo, censite e misurate dalle indagini nazionali e internazionali sui livelli di competenza degli adulti.

Questo genere di indagini sull'alfabetizzazione degli adulti prese piede negli anni Novanta ${ }^{6}$ del secolo scorso, quando Canada, Stati Uniti e OCSE diedero avvio a una raccolta di dati per integrare quan-

6 Per una rassegna esaustiva sulle indagini internazionali e nazionali sulle competenze alfabetiche degli adulti si vedano i lavori di Spano (2020) e Ligas (2021). 
to già misurato e valutato dall'UNESCO (Wagner 2011), nell'ottica più ampia di effettuare uno studio comparativo basato sulla misurazione e sulla valutazione esterna sul rendimento in particolare per specifiche abilità, come la comprensione della lettura.

L'indagine più recente, con caratteristiche leggermente diverse dalle precedenti indagini, è stata condotta dall'OCSE: è il Programme for International Assessment of Adult Competencies (PIAAC), con l'obiettivo primario di valutare le competenze chiave, cognitive e lavorative della popolazione tra i 16 e 65 anni di età, finalizzate a un'integrazione efficace nel tessuto sociale ed economico contemporaneo a partire dall'istruzione, in particolare per le fasce di popolazione considerate 'a rischio' di esclusione sociale, offrendo ai governi uno strumento per l'attuazione di politiche nazionali e interventi mirati.

Lo strumento di rilevazione è composto da un questionario sulle variabili socio-anagrafiche e da test cognitivi ideati dall'OCSE, poi tradotti e adattati al contesto nazionale dai singoli Paesi partecipanti. I test cognitivi mirano all'analisi di abilità e competenze utilizzabili sia sul lavoro che nella vita quotidiana, riguardanti gli ambiti della literacy, della numeracy e del problem solving in ambienti tecnologicamente avanzati, così come definiti nell'Indagine PIAAC. Alcuni dei Paesi coinvolti nel primo round di somministrazioni (fra cui l'Italia) non hanno utilizzato i test riguardanti il problem solving, optando per quelli relativi ai reading components, un fascicolo di prove che ha l'obiettivo di discriminare la condizione di illetteratismo. I reading components ${ }^{7}$ si basano secondo l'OCSE su:

the basic set of decoding skills that are essential for extracting meaning from written texts: knowledge of vocabulary (word recognition), the ability to process meaning at the level of the sentence, and fluency in reading passages of text. Skilled readers are able to undertake these types of operations automatically. (OECD 2019a)

Fra queste prove rientra ad esempio la verifica della comprensione del testo stampato (print vocabulary), ovvero la decodifica e riconoscimento visuale delle parole (Mineo, Amendola 2018), un abbinamento tra parola scritta e immagine. Altri esempi di prove per coloro che possiedono basse competenze alfabetiche funzionali sono l'elaborazione del senso di una frase (sentence processing) e la comprensione di passaggi all'interno di brani semplici (passage comprehension).

La disamina sulle prove di misurazione delle competenze degli adulti mette in evidenza la complessità delle richieste che le società contemporanee pongono agli individui.

7 Una descrizione approfondita dei reading components è presente in: https://www. oecd.org/skills/piaac/Reading\%20Components\%20Sample\%20Items.pdf. 
Nell'ambito di tale complessità pare necessario non solo lavorare sull'accrescimento delle competenze degli individui, ma anche e soprattutto sulla loro sensibilizzazione in relazione alla necessità imprescindibile di possedere tali competenze e sulla loro motivazione (Caon 2019), non solo per poter svolgere azioni quotidiane (cercare una casa, usare i mezzi pubblici, lavorare, avere dei contatti con l'ambiente scolastico dei figli) ma anche e soprattutto per vivere praticando quella 'cittadinanza attiva' alla quale esortano documenti e organismi europei, ovvero il possesso dei livelli minimi di istruzione che consentono lo sviluppo di una coscienza civica che possa espletarsi nelle quattro dimensioni della partecipazione alla vita politica, alla società civile, alla vita di comunità e nell'adesione ai valori universali quali la democrazia, i diritti umani, il dialogo interculturale (CRELL 2006). Per dirla con le parole di Tullio De Mauro $(2018,23)$ : «il possesso di questo bagaglio linguistico minimo è una necessità funzionale per una società complessa che voglia essere pur minimamente democratica».

Per coloro (sia apprendenti di L2 che parlanti nativi) che hanno intrapreso il percorso verso l'alfabetizzazione, è comune usare la metafora del passaggio dalla cecità alla visione nel momento in cui si impara a leggere, scrivere e a comprendere i numeri: di colpo gli ambienti, le immagini, la percezione di sé e del mondo cambiano radicalmente e diventano intelligibili, come riporta D'Agostino (2016, 16-17) in relazione alle interviste ad un uomo palermitano e a una giovane marocchina inseriti in un percorso di scolarizzazione e apprendimento della letto-scrittura: «Mi sentivo come avere le gambe, avere le mani, avere la testa per potere ragionare senza però potere guardare. Gli occhi li avevo per poter guardare, però guardavo ma non vedevo niente [...]. Ho riacquistato la vista, come ho imparato a leggere e scrivere» (G., 32 anni, Palermo). L'esperienza è condivisa con A., 16 anni, marocchina: «Per strada guardo, visto questo, visto quello, ricordo dove passato. [...] Vedo niente, vedo disegni belli, nelle strade, solo vedere, leggi niente. Sono arrabbiata dentro perché io voi leggere».

La descrizione delle competenze di letto-scrittura ha portato alla definizione di tipologie di apprendenti distinte sia per bisogni di apprendimento sia per strategie di avvicinamento alla L2. In diversi studi (Tarone, Bigelow, Hansen 2009; Van de Craats, Kurvers, YoungScholten 2006) è stato infatti dimostrato come l'apprendimento della L2 sia fortemente influenzato negli adulti dalle competenze pregresse: individui che non sono stati scolarizzati nella L1 presentano maggiori difficoltà nell'apprendimento della L2 dal momento che il sistema cognitivo viene strutturato anche attraverso le attività legate al riconoscimento e all'utilizzo della parola scritta. Come affermato in Brichese $(2019,31)$ è necessario anche ribadire l'importanza dello sviluppo della consapevolezza fonologica sia per lo sviluppo della competenza lessicale sia per le abilità tecniche di lettura e scrittura. 
Come dimostra l'esperienza riportata da D'Agostino, il problema dell'analfabetismo purtroppo non fa parte nemmeno nelle società occidentali di un passato ormai superato, ma è ancora un problema da affrontare e risolvere. Si pensi ad esempio alla situazione dell'Italia, in cui il 5\% della popolazione risulta ancora analfabeta strumentale (cioè chi non ha mai imparato a leggere e scrivere), mentre oltre il $28 \%$ si situa al di sotto del livello minimo di competenze in literacy, secondo i dati emersi dall'indagine ALL (Adult Literacy and Life Skills) del 2006 (Gallina 2006) e commentati in De Mauro (2018).

$\mathrm{Nel}$ contesto europeo, di cui l'Italia rappresenta solo un esempio, l'arrivo di flussi migratori ingenti da Paesi in situazioni di conflitto o instabilità, in cui i tassi di alfabetizzazione sono bassi o molto bassi, ha portato negli ultimi anni a un notevole incremento di studenti analfabeti nelle classi dei corsi di L2 destinati ad adulti. Il Rapporto Annuale SPRAR (2016) mette in evidenza come in Italia quasi il 25\% dei richiedenti asilo e rifugiati siano stati inseriti in corsi di pre-alfabetizzazione per l'apprendimento delle competenze di letto-scrittura; nel Rapporto Annuale SPRAR /SIPROIMI 2018 i beneficiari non in possesso di titolo di studio risultano essere il $12 \%$, e il $14,7 \%$ ha seguito corsi di pre-alfabetizzazione mentre il rapporto REACH commissionato dall'UNICEF (in D’Agostino 2018) sottolinea come negli sbarchi di minori stranieri non accompagnati (MSNA) siano presenti percentuali di ragazzi analfabeti riconducibili a quelle presenti nei Paesi di origine (D’Agostino 2017); in Germania, invece, secondo il rapporto Literacy Training and German Language Acquisition Among Refugees: Knowledge of German and the Need for Support Among Integration Course Attendees Learning a Second Alphabet and Those with No Literacy Skills (Scheible 2018), all'incirca il 34\% dei rifugiati era in grado di leggere e scrivere in alfabeto latino, il 51\% aveva acquisito le competenze di letto-scrittura in una lingua con un altro alfabeto - e il 15\% non possedeva alcuna competenza in letto-scrittura; di questi, meno di un quinto aveva preso parte a un corso di integrazione; infine, un'indagine OIM risalente al 2016 ha messo in evidenza come, fra i migranti neorrivati in Italia, di cui il 60\% è rappresentato da richiedenti asilo, il 10\% risultava completamente analfabeta, il $20 \%$ non aveva completato nessun ciclo di scuola e il $29 \%$ aveva completato la scuola primaria (IOM 2016).

Come è evidente, quindi, i profili presenti spaziano dal caso di apprendenti che non hanno mai avuto contatti con la lingua scritta, definiti pre-alfabeti, perché nati e cresciuti all'interno di comunità in cui la lingua non è veicolata dal codice scritto, come può verificarsi per individui provenienti da alcuni contesti rurali di Asia, America, Africa, Oceania, ad apprendenti provenienti da società in cui la lingua scritta coesiste con la lingua orale ma che non hanno avuto accesso alla scolarizzazione e quindi mostrano una mancanza di competenze in letto-scrittura sia in lingua madre sia nella lingua target 
(definiti analfabeti strumentali, da suddividere in base alla L1, alfabetica o non alfabetica); o ancora ai cosiddetti analfabeti funzionali o di ritorno, definizione all'interno della quale si annoverano coloro che non hanno avuto una scolarizzazione adeguata sia in termini di tempo trascorso sui banchi sia di qualità dell'insegnamento o che hanno perso, per impossibilità ad esercitarle, le competenze di letto-scrittura. In aggiunta, bisogna comunque tener conto anche del fatto che queste diverse tipologie di apprendenti possono avere contatti più o meno frequenti con i contesti di arrivo (in termini di relazioni sociali, occasioni di usare e praticare la lingua) e ciò svolge naturalmente un ruolo fondamentale nella velocità con cui il processo di alfabetizzazione può essere portato a compimento o presentare progressi significativi.

Quest'ultimo punto risulta di particolare interesse: il processo di alfabetizzazione viene oggi concepito infatti come un processo collettivo, al quale concorrono in diversa misura svariati attori. In primis, l'individuo adulto che, portatore di vissuti, di competenze linguistiche orali spesso in diverse lingue, di esperienze professionali e personali decide di intraprendere un percorso che faciliterà la sua esistenza nella società di arrivo e rivoluzionerà la sua vita; in seconda battuta, gli attori sociali (reti di educazione formale e non formale, reti amicali) che, interagendo con l'apprendente, offrono occasioni di apprendimento spontaneo e guidato e riflessioni che rendono utile ciò che l'individuo ha appreso, attribuiscono senso agli sforzi e alla tensione dell'individuo verso l'obiettivo dell'alfabetizzazione attraverso la negoziazione dei significati. L'aumento delle motivazioni (strumentali, integrative e intrinseche), insieme alla riflessione sull'utilità del percorso intrapreso nelle situazioni di vita reale rendono tale percorso verso l'alfabetizzazione sociale e significativo: sociale nel senso di condiviso con i membri della comunità di arrivo (si veda a tal proposito anche il modello «a mediazione sociale» proposto da Caon in Brichese, Tonioli 2017) e significativo perché utile a intraprendere e gestire relazioni nei diversi domini, in una relazione circolare virtuosa.

Per raggiungere questo obiettivo è possibile e necessario appellarsi al concetto di competenza parziale, sviluppato nell'ambito delle politiche per il plurilinguismo promosse dal Consiglio d'Europa già introdotte nel capitolo 3: così come gli apprendenti di L2 devono poter usare la propria competenza linguistica, seppur 'imperfetta' e in divenire, per potersi districare nelle diverse situazioni della vita quotidiana, allo stesso modo coloro che hanno intrapreso un percorso di alfabetizzazione devono poter sperimentare nella vita reale quanto appreso, riconoscendo testi scritti nei luoghi e negli ambienti che frequentano, usando e valorizzando, anche se limitatamente all'inizio, i primi progressi nella scrittura.

L'inserimento di studenti analfabeti (anche se a livelli diversi, come accennato in precedenza) nelle classi di L2 pone quindi delle sfide edu- 
cative importanti, sia ai docenti che conducono i corsi sia alle istituzioni che sono incaricate di realizzare questo delicato compito, la prima delle quali è proprio la corretta identificazione del profilo dell'apprendente. A tal proposito, si ripropone qui la schematizzazione dei livelli comprensiva dei livelli pre-alfa elaborata da Minuz, Borri (2016).

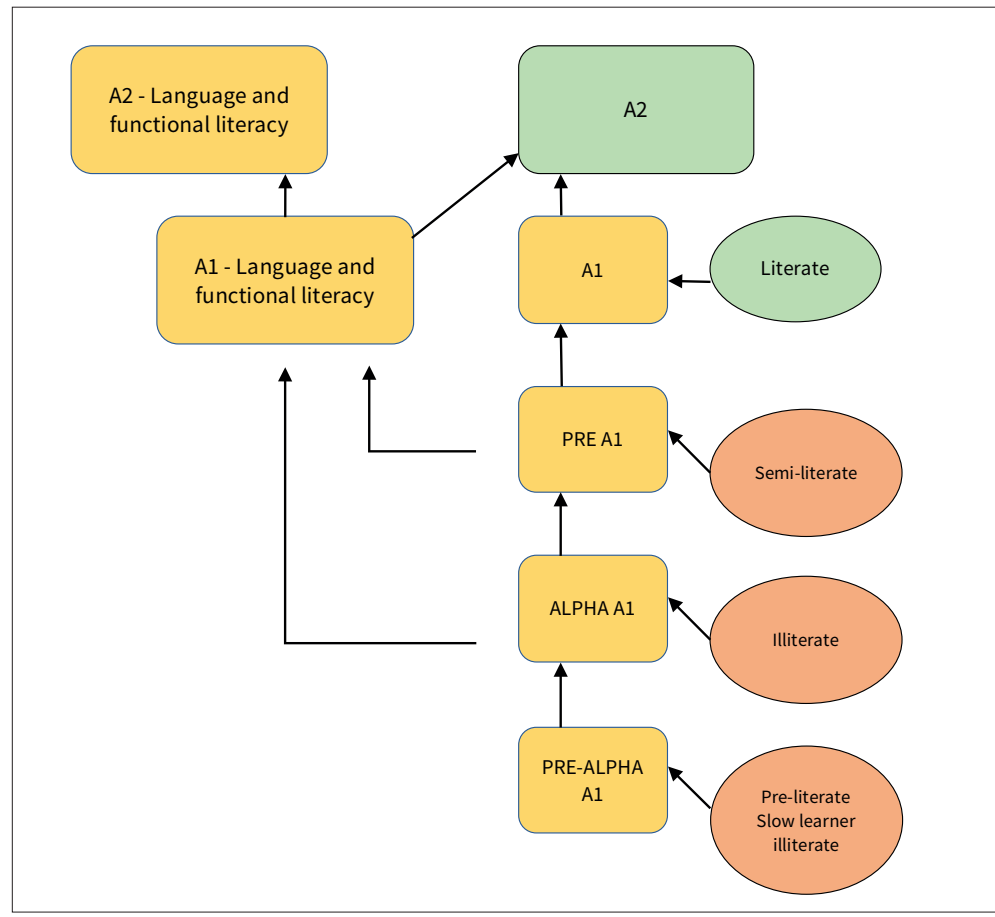

Figura 1 Schematizzazione dei livelli comprensiva dei livelli pre-alfa (tratto da Minuz, Borri 2016, 228

Nella tabella è possibile osservare la distinzione tra i quattro livelli (literacy profiles) che si distinguono per competenze in lettoscrittura: dal Pre-Alfa, in cui rientrano coloro che provengono da società in cui non è presente la lingua codificata in forma scritta 0 persone con L1 tipologicamente distanti, con sistemi alfabetici diversi e scarsamente scolarizzate, all'A1, in cui rientrano profili di apprendenti pienamente alfabetizzati. Per ciascuno di questi profili è fondamentale pianificare interventi formativi in L2 con obiettivi didattici differenziati e con un numero di ore adeguato, secondo standard condivisi.

Pur rappresentando in Europa una fetta sempre più ampia dell'offerta didattica in L2, l'offerta di corsi di alfabetizzazione presenta infatti caratteristiche molto diversificate a seconda dei Paesi. 
In Italia sono i CPIA (Centri Provinciali per l'Istruzione degli Adulti) a rappresentare il baluardo dell'offerta didattica a questa fascia di popolazione, ${ }^{8}$ attraverso i corsi specifici di alfabetizzazione preA1 della durata di 25 ore, mentre in altri Paesi, come la Svezia, la Norvegia, la Danimarca, l'Olanda esistono centri e percorsi dedicati esclusivamente a questa tipologia di pubblico. In Germania, invece, i cittadini a bassa scolarizzazione hanno il diritto/dovere di seguire corsi di 1.200 ore per raggiungere il livello B1 (Scheible 2018); ${ }^{9}$ dal 2005 i corsi di integrazione offerti dal Federal Office for Migration and Refugees (BAMF) sono stati lo strumento principale di supporto a migranti per l'apprendimento del tedesco, con un sistema modulare per determinare il numero di ore necessario a ciascun profilo di apprendente, secondo la tabella qui riportata.

8 I bisogni degli studenti con scarsa o scarsissima alfabetizzazione non erano stati rappresentati all'interno del DPR 4.10.2012, che istituisce i CPIA in luogo dei precedenti CTP (Centri Territoriali Permanenti): il primo gradino di istruzione per gli adulti è considerato la licenza media, tuttavia molti studenti non possiedono neppure un livello Pre-A1. Per ovviare a tale grave mancanza è stato lanciato un appello nel 2013, firmato da moltissimi docenti di italiano L2 ad adulti, da Paola Casi, docente ed esperta di analfabetismo, che paragona la necessità di alfabetizzazione di queste fasce di utenti vulnerabili alla necessità di un intervento di pronto soccorso: un'urgenza inderogabile, alla quale le istituzioni devono porre rimedio attraverso canali e corsi preferenziali. Come segnalato in un articolo di Mari D'Agostino (2017), dopo cinque anni questa situazione non si era modificata. Solo nelle Linee guida per la progettazione dei Piani regionali per la formazione civico linguistica dei cittadini di Paesi Terzi, finanziati a valere sul FAMI - OS 2 - ON 2 Azioni formative specifiche, percorsi sperimentali, sono comparse delle indicazioni in merito.

9 Il corso di integrazione generale mira a permettere ai migranti di acquisire competenze linguistiche equivalenti al livello B1 del Quadro comune europeo di riferimento per le lingue (QCER o CEFR). Per completare con successo il corso di integrazione generale e l'acquisizione di competenze linguistiche di livello B1, i partecipanti devono essere in grado di leggere l'alfabeto latino e avere una formazione di base prima di iniziare il corso. Per rispondere alle esigenze specifiche che alcuni cittadini immigrati, la gamma dei corsi di integrazione è stata ampliata dal 2005 e comprende altri corsi più specifici adattati a particolari gruppi target, fra i quali corsi per analfabeti e, dal febbraio 2017, corsi per persone che imparano un secondo alfabeto, come si può osservare nella tabella. I corsi che includono la formazione all'alfabetizzazione includono più ore di lezione lezioni del corso di lingua generale (fino a 900 o, per il corso per analfabeti, fino a 1.200 ore). L'obiettivo (realistico) del corso per analfabeti è quindi quello di aiutare la maggioranza dei partecipanti ad acquisire competenze linguistiche equivalenti al livello A2.2 (BAMF 2015). 
Tabella 1 Lezioni di lingua nel corso di integrazione generale, il corso per persone analfabete e persone che imparano un secondo alfabeto (fonte: Scheible 2018)

\begin{tabular}{|c|c|c|c|}
\hline & General integration course & $\begin{array}{c}\text { Course for people learning a second } \\
\text { alphabet }\end{array}$ & Course for illiterate people \\
\hline $\begin{array}{l}\text { Legal precon- } \\
\text { ditions }\end{array}$ & \multicolumn{3}{|c|}{$\begin{array}{l}\text { For foreign nationals with a permanent residence status and German nationals with particular integration needs and a mi- } \\
\text { grant background; since } 24 \text { October } 2015 \text { also for asylum applicants with good prospects to remain (currently from Syria, Iraq, } \\
\text { Iran, Eritrea, Somalia), persons whose removal has been suspended pursuant to Section } 60 \text { a subs. } 2 \text { sentence } 3 \text { of the Resi- } \\
\text { dence Act, and holders of a residence permit pursuant to Section } 25 \text { subs. } 5 \text { of the Residence Act. }\end{array}$} \\
\hline \multicolumn{4}{|c|}{ Language course } \\
\hline $\begin{array}{l}\text { Target group: } \\
\text { linguistic } \\
\text { preconditions }\end{array}$ & $\begin{array}{l}\text { Functionally literate in the Latin } \\
\text { alphabet } \\
\text { Have reading, writing and learning } \\
\text { experience }\end{array}$ & \begin{tabular}{|l|} 
People learning a second alphabet, \\
i. e. who can read and write one or \\
more languages which do not use the \\
Latin alphabet \\
= Acquire additional literacy in the Latin \\
alphabet \\
- Usually have substantial reading, writ- \\
ing and learning experience
\end{tabular} & $\begin{array}{l}\text { People without literacy skills in any } \\
\text { alphabet, i. e. who are not or insuffi- } \\
\text { ciently literate in either the Latin or } \\
\text { another alphabetUsually have no or } \\
\text { only rudimentary reading, writing and } \\
\text { learning experience }\end{array}$ \\
\hline Goal & $\begin{array}{l}\text { Language level B1 (speaking, under- } \\
\text { standing, writing and reading): } \\
\text { people can manage everyday situa- } \\
\text { tions and are able to express them- } \\
\text { selves }\end{array}$ & $\begin{array}{l}\text { Language level B1 (see general inte- } \\
\text { gration course) }\end{array}$ & $\begin{array}{l}\text { Overall target: level B1 } \\
\text { For most, level A2.2 is realistic } \\
\text { For primary illiterates, the minimum } \\
\text { goal is level A2.1 }\end{array}$ \\
\hline Extent & $\begin{array}{l}600 \text { lessons (additional special cours- } \\
\text { es, for example an intensive course } \\
\text { with } 400 \text { lessons) } \\
300 \text { lessons may be repeated if par- } \\
\text { ticipants attend regularly and do not } \\
\text { achieve level } B 1 \text { in the test }\end{array}$ & $\begin{array}{l}900 \text { lessons (standard } 600 \text { lessons, } \\
\text { plus a further } 300 \text { lessons if partici- } \\
\text { pants attended regularly) }\end{array}$ & $\begin{array}{l}1,200 \text { lessons (standard } 900 \text { lessons, } \\
\text { plus a further } 300 \text { lessons if partici- } \\
\text { pants attended regularly) }\end{array}$ \\
\hline $\begin{array}{l}\text { Course } \\
\text { structure }\end{array}$ & $\begin{array}{l}\text { Basic: } 300 \text { lessons } \\
\text { Advanced: } 300 \text { lessons }\end{array}$ & $\begin{array}{l}\text { Basic: } 300 \text { lessons } \\
\text { Advanced A: } 300 \text { lessons } \\
\text { = Advanced B: } 300 \text { lessons } \\
\text { Intensive introduction to the Latin } \\
\text { alphabet, followed by the language } \\
\text { course }\end{array}$ & $\begin{array}{l}\text { Basic: } 300 \text { lessons } \\
\text { Advanced A: } 300 \text { lessons } \\
\text { Advanced B: } 300 \text { lessons } \\
\text { Advanced C: } 300 \text { lessons } \\
\text { Make participants functionally literate as } \\
\text { far as possible, teach them German at the } \\
\text { same time }\end{array}$ \\
\hline $\begin{array}{l}\text { Assessment } \\
\text { test }\end{array}$ & \multicolumn{3}{|c|}{ Uniform assessment test at the beginning of the course with integrated alpha component } \\
\hline \multirow[t]{2}{*}{$\begin{array}{l}\text { Final } \\
\text { examination }\end{array}$} & \multicolumn{3}{|c|}{ Completion of language course by graded language test “German test for immigrants”(DTZ) } \\
\hline & \multicolumn{3}{|c|}{ Orientation course } \\
\hline Extent & \multicolumn{3}{|c|}{100 lessons in politics and culture } \\
\hline $\begin{array}{l}\text { Final } \\
\text { examination }\end{array}$ & \multicolumn{3}{|c|}{ Completion of orientation course by the "Life in Germany" test } \\
\hline
\end{tabular}

Tuttavia, la letteratura recente mette in evidenza la necessità di tracciare un profilo specifico degli analfabeti/bassamente scolarizzati, per individuare le variabili relative alla literacy in L1 che impattano sull'apprendimento della L2, offrendo agli insegnanti strumenti specifici per intervenire su di esse. È importante evidenziare delle buone pratiche esistenti in tal senso, come ad esempio il test del Centro Itastra dell'Università degli Studi di Palermo per indagare la litera$c y$, elaborato nelle diverse lingue dei migranti: arabo, bambara, bangla, francese, inglese, spagnolo, mandinka, wolof e pular (D’Agosti- 
no 2017); la proposta di un test d'ingresso FAMI elaborata da Rocca (2019) con il contributo di Caon, Brichese e con il contributo dell'ALTE, consistente in un'unica prova che contempli l'intervista conoscitiva per l'analisi dei bisogni e l'accertamento delle competenze alfabetiche e linguistiche, con una banda di oscillazione che vada dall'analfabetismo totale al livello B1; il test multilingue per la verifica delle competenze alfabetiche in L1 sviluppato in Belgio e testato su un campione di 351 richiedenti asilo (Hooft, Schiepers, Vandommele 2021); o ancora il test basato su autodichiarazioni riguardanti le quattro abilità di base in inglese, francese e tedesco somministrato in Germania (Scheible 2018). ${ }^{10}$

$\mathrm{Al}$ di là delle distinzioni relative alle competenze di literacy, sulla scorta delle osservazioni condotte in Shapiro et al. (2018), è opportuno concentrare l'attenzione su una particolare fascia di apprendenti, definita refugee-background students.

Il punto di partenza di questa riflessione, contenuta nel recente volume Educating Refugee-background Students (Shapiro et al. 2018), risiede nella definizione della condizione di rifugiato.

Coniata nell'ambito della Convenzione di Ginevra elaborata durante la Seconda guerra mondiale per tutelare legalmente coloro che migravano forzosamente dall'Europa, questa etichetta raduna oggi persone provenienti da molte altre aree del mondo, in fuga dal proprio Paese per conflitti e, sempre di più, per crisi e catastrofi naturali provocate dal cambiamento climatico. Per questi individui in fuga, alla ricerca di protezione in un Paese diverso da quello di origine, la prima prospettiva è quella di ottenere lo status di richiedenti asilo, condizione in seguito alla quale vengono presi in carico dall'UNHCR e trasferiti nei Paesi cosiddetti di primo asilo, nella maggior parte dei casi in campi profughi. La determinazione eventuale dello status giuridico di rifugiato avviene in genere dopo molto tempo e in una percentuale ridottissima di casi; lo step successivo può consistere in un rimpatrio volontario, nel caso in cui nel Paese di origine si siano ristabilite delle condizioni di sicurezza, oppure la possibilità di godere del resettlement, ${ }^{11}$ un trasferimento più stabile e con una prospettiva più a lungo termine in un altro Paese, questa riguarda una percentuale ancora minore (l'1\%) di individui, che devono però rientrare in una delle sette categorie protette che possono beneficiare di questa misura.

10 Sebbene il dibattito tra gli studiosi sia ancora aperto, è evidente che sia i test oggettivi che i test cosiddetti soggettivi presentano vantaggi e svantaggi. In particolare, il test oggettivo risulta di difficile realizzazione perché dovrebbe essere redatto nelle diverse lingue dei migranti ai quali si intende sottoporlo; il test soggettivo rischia di fornire un quadro distorto della realtà, anche se diversi studi dimostrano una corrispondenza abbastanza precisa fra le autodichiarazioni fornite e il livello che emerge dalla somministrazione di un test oggettivo (Edele 2015).

11 Per una definizione di resettlement si vedano i contributi di Grosso e Da Pra (2021). 
Pur essendo un processo lungo e oneroso, il resettlement è ovviamente l'unica strada che permette ai rifugiati di poter avviare un processo di integrazione a lungo termine nel Paese di destinazione definitiva, con il rilascio di un permesso di soggiorno che abiliti alla ricerca di un lavoro, una condizione abitativa stabile e l'inserimento, sia per i minori sia per gli adulti, all'interno di contesti formativi come scuole e centri di educazione per gli adulti. Secondo i dati dell'UNHCR (2016) ${ }^{12}$ circa 160.000 persone sono state beneficiarie di resettlement in tutto il mondo, in particolare in Stati che hanno offerto una maggiore disponibilità di permessi di soggiorno per persone rientranti in questa categoria. Esistono Paesi con una forte tradizione di accoglienza di immigrati attraverso il resettlement, come gli Stati Uniti, il Canada o l'Australia, mentre molti altri solo recentemente hanno intrapreso tale percorso. Fra questi, molti Paesi del sud dell'Europa (come l'Italia e la Spagna) che, per la loro vicinanza geografica ai luoghi di origine degli individui immigrati, sono anche in molti casi Paesi di primo asilo, considerati la porta d'accesso dei flussi migratori al continente europeo.

È possibile affermare che, sia nel caso di resettlement sia più in generale nelle prime fasi del processo di immigrazione, i contesti formativi giocano un ruolo fondamentale nella prima accoglienza e nella fase di integrazione sociale e culturale più a lungo termine. Essi rappresentano infatti uno degli ambiti in cui avviene l'integrazione (Webb et al. 2016), che passa per la costruzione di relazioni tra individui coinvolti nel processo formativo: studenti, insegnanti, operatori scolastici. Le scuole, i centri di istruzione ed educazione degli adulti sono l'ambiente in cui gli individui tornano ad essere riconosciuti nella loro specificità, come persone; in cui la valorizzazione delle competenze pregresse degli individui permette di ragionare non adottando una visione deficitaria degli individui stessi, ma costruendo invece a partire dal bagaglio dei vissuti culturali, linguistici e molto spesso traumatici di cui questi cittadini sono portatori. I contesti educativi infatti non si limitano ad essere contenitori di corsi che erogano nozioni ma al contrario danno essi stessi forma all'esperienza dell'integrazione (Virgilio 2018, 11):

Sono i contesti, infatti, a costituire le cornici di senso entro cui gli esiti dei processi educativi vanno valutati, non solo in termini di apprendimento, ma soprattutto in termini di produzione di processi di cittadinanza. Nei CPIA [...] gli studenti con background migratorio possono imparare una con-cittadinanza ancorata al contesto nazionale e insieme aperta a un mondo sempre più grande, interdipendente, interconnesso. [...] si 'allenano' a convivere in una pluralità diffusa. [...] Famiglie e comunità con storie diverse 
possono imparare a conoscersi, superare le reciproche diffidenze, sentirsi responsabili di un futuro comune.

I contesti formativi risultano quindi essere i luoghi in cui vengono implementate le didattiche delle cosiddette nuove literacies che caratterizzano la società della conoscenza, quelle sfaccettature dell'alfabetizzazione che sono basate sulla multimodalità e «sui diversi tipi di significati che si manifestano nei contesti sociali e culturali» (Kalantzis, Cope in Zoletto 2018, 35).

All'interno dei contesti formativi, nei Paesi sia di primo asilo sia di destinazione finale, come l’Italia, la Spagna, la Grecia, il pubblico dei corsi di L2 è quindi variegato, con i tradizionali fattori che caratterizzano la classe ad abilità differenziate, ovvero diversi gradi di scolarizzazione, diversi stili cognitivi, diverse tipologie di intelligenze, stili cognitivi e di apprendimento, repertori linguistici compositi, in aggiunta alle motivazioni all'apprendimento della lingua target, radicalmente diverse.

Per i richiedenti asilo o per i rifugiati in attesa di assegnazione di una destinazione finale, infatti, le motivazioni sono prevalentemente strumentali: in mancanza della possibilità di avere un progetto migratorio a lungo termine, con la possibilità di integrarsi stabilmente con la comunità di arrivo, la lingua viene appresa quasi esclusivamente per poter sopravvivere nella quotidianità, prescindendo da un reale interesse a costruire relazioni stabili e durature nei contesti di vita. Per coloro che invece vivono nella comunità di arrivo con un progetto migratorio stabile, di inserimento e inclusione sociale sotto tutti i punti di vista, le motivazioni sono, oltre che strumentali, integrative, specie nel caso in cui le aspettative verso il contesto di arrivo non siano irrealistiche (cf. Grosso 2021).

È però importante ricordare che il minimo comune denominatore tra queste due diverse tipologie di apprendenti è costituito dal peso che eventi traumatici possono aver avuto nella vita di questi individui, che, come ricordato in precedenza, provengono da situazioni catastrofiche / di conflitto e possono aver affrontato periodi e contesti di transizione caratterizzati dalla violenza o dalla deprivazione economica, sociale e culturale (Dryden-Peterson 2015). In ogni caso, l'utilizzo della definizione refugee background students è consigliabile, come afferma Shapiro (in Shapiro et al. 2018), proprio perché permette di immaginare e descrivere questa tipologia di apprendente non in termini deficitari (deficit di competenze, deficit di relazioni, deficit di agentività) ma in termini di potenziale polilinguistico (Pennycook 2012; D’Agostino, Sorce 2016) o di competenze informali da considerare «funds of knowledge» (Shapiro et al. 2018) che questi individui possono dispiegare, una volta nelle condizioni di potersi emancipare dalla condizione transitoria di fuga che ha caratterizzato la loro esistenza fino al momento dell'arrivo nel Paese di destinazione. Riferendosi soltanto al possesso di un repertorio linguistico varie- 
gato, individui magari analfabeti che tendono ad essere considerati privi di competenze linguistiche parlano in realtà quattro/cinque lingue, usate in diversi contesti comunicativi e con diversi interlocutori; questo repertorio si arricchisce durante il percorso migratorio, nel lungo transito per Paesi europei ed extraeuropei prima dell'arrivo alla destinazione finale. È questo un dato riscontrato con grande frequenza all'interno di un contesto superdiverso per eccellenza, in cui si concentrano dinamiche di 'supercontatto' nella convivenza forzata tra individui dai molteplici retroterra linguistico-culturali: il contesto penitenziario (Benucci, Grosso 2015; 2017).

All'interno del contesto penitenziario, il problema dell'analfabetismo è drammaticamente presente e riguarda sia i detenuti stranieri che i nativi. Non a caso, la mancata scolarizzazione, la povertà linguistica nel contesto sociale di appartenenza, il precoce abbandono scolastico, l'appartenenza a una famiglia con bassi livelli di istruzione, la mancanza di sostegno educativo, la difficoltà nella lettura, i bassi livelli di educazione formale e quindi le scarse competenze di literacy (intesa nel senso ampio del termine) sono considerate «fattori criminogeni educativi» (Savoia 2013) dato che, pur non dimostrando un legame causale tra la mancanza di un percorso educativo adeguato e la possibilità di intraprendere un percorso deviante, evidenzia come la prima condizione possa favorire l'insorgere della seconda.

Tabella 2 Fattori criminogeni educativi

\begin{tabular}{|c|c|c|}
\hline & Fattori criminogeni educativi & Altri fattori criminogeni \\
\hline \multirow[t]{5}{*}{ Fattori statici } & $\begin{array}{l}\text { Mancanza di sostegno } \\
\text { educativo da parte dei genitori }\end{array}$ & Mancanza di cure nell'infanzia \\
\hline & $\begin{array}{l}\text { Povertà linguistica nel contesto } \\
\text { sociale di appartenenza }\end{array}$ & Abusi subiti nell'infanzia \\
\hline & $\begin{array}{l}\text { Povertà d'insegnamento nei } \\
\text { primi anni di scolarizzazione }\end{array}$ & $\begin{array}{l}\text { Problemi di vista e udito } \\
\text { nell'infanzia }\end{array}$ \\
\hline & Precoce abbandono scolastico & Carriera criminale \\
\hline & $\begin{array}{l}\text { Appartenenza a una famiglia } \\
\text { con bassi livelli d'istruzione }\end{array}$ & \\
\hline \multirow[t]{6}{*}{$\begin{array}{l}\text { Fattori } \\
\text { dinamici }\end{array}$} & $\begin{array}{l}\text { Bassi livelli di educazione } \\
\text { formale }\end{array}$ & Uso di droghe e alcool \\
\hline & Povertà linguistica & Mancanza di fissa dimora \\
\hline & $\begin{array}{l}\text { Deficit dell'apprendimento } \\
\text { e dell'attenzione }\end{array}$ & Disoccupazione \\
\hline & Dislessia & Reti sociali di sostegno \\
\hline & Difficoltà nella lettura & Ansia o depressione \\
\hline & & Equilibrio mentale \\
\hline
\end{tabular}

Fonte: Savoia 2013, 13 
L'indagine NAAL (National Assessment of Adult Literacy), condotta per la prima volta nel 1992, aggiornata nel 2003 e ripetuta nel 2014, valuta le abilità e le competenze di alfabetizzazione della popolazione adulta degli Stati Uniti, con la valutazione delle competenze di specifici segmenti della popolazione tra i quali la popolazione carceraria (Haigler et al. 1994). Alla valutazione del 2003 hanno partecipato circa 1.200 adulti, provenienti da 107 prigioni (incluse 12 prigioni federali) di 31 Stati, con l'obiettivo di fornire a policymaker e attori del contesto educativo/formativo dati demografici e di rendimento per la popolazione carceraria, a confronto con i dati NAAL sulla popolazione adulta in generale, per promuovere programmi di alfabetizzazione e di educazione degli adulti più efficaci per i detenuti.

L'indagine NAAL in contesto carcerario ${ }^{13}$ prevede la somministrazione:

1. di un questionario di base, che raccoglie informazioni sulle caratteristiche demografiche e di altro tipo, associate al livello di alfabetizzazione (ad esempio, età, etnia, sesso, lingua, istruzione, formazione sul lavoro, uso della tecnologia, condizioni e attività relative alla salute e pratiche di alfabetizzazione); per il questionario della popolazione carceraria vengono raccolte le informazioni sulle esperienze in carcere, come i programmi speciali e le attività di gruppo, i reati commessi, le storie criminali, gli incarichi di lavoro, l'accesso alla biblioteca e i programmi di istruzione intrapresi dopo l'incarcerazione;

2. domande di screening di base, ovvero un test preliminare per determinare se gli intervistati hanno sufficienti competenze di alfabetizzazione per completare la valutazione 'NAAL principale'; a coloro che hanno prestazioni molto basse in questi compiti viene somministrata un'altra tipologia di test chiamata Adult Literacy Supplemental Assessment (ALSA), invece del test NAAL principale;

3. test di valutazione delle competenze NAAL o ALSA; il test NAAL principale valuta le prestazioni di alfabetizzazione basate su compiti (azioni quotidiane come identificare le informazioni necessarie in un orario dell'autobus) mentre l'ALSA misura le abilità di base di alfabetizzazione a livello di parole e, per la prima volta, fornisce informazioni molto accurate sulle abilità e le debolezze di alfabetizzazione degli adulti meno alfabetizzati. Le indicazioni orali e le domande sono fornite sia in inglese che in spagnolo e le risposte orali possono essere in entrambe le lingue, ma il materiale che gli intervistati devono leggere è in inglese;

13 Per un approfondimento sul test NAAL si veda: https://nces.ed.gov/naal/fct_ prison.asp. 
4. infine, il Fluency Addition to NAAL (FAN) misura la fluidità di lettura orale di tutti i partecipanti utilizzando un software di riconoscimento vocale per valutare la capacità degli adulti di decodificare e riconoscere le parole e di leggere ad alta voce con scioltezza; le indicazioni orali e le domande sono fornite in inglese o spagnolo, ma le risposte devono essere in inglese.

I dati raccolti confermano l'ipotesi secondo la quale la popolazione dei detenuti ha competenze di alfabetizzazione e di calcolo più basse rispetto alla popolazione generale (literacy e numeracy), come mostrato nella figura 2.

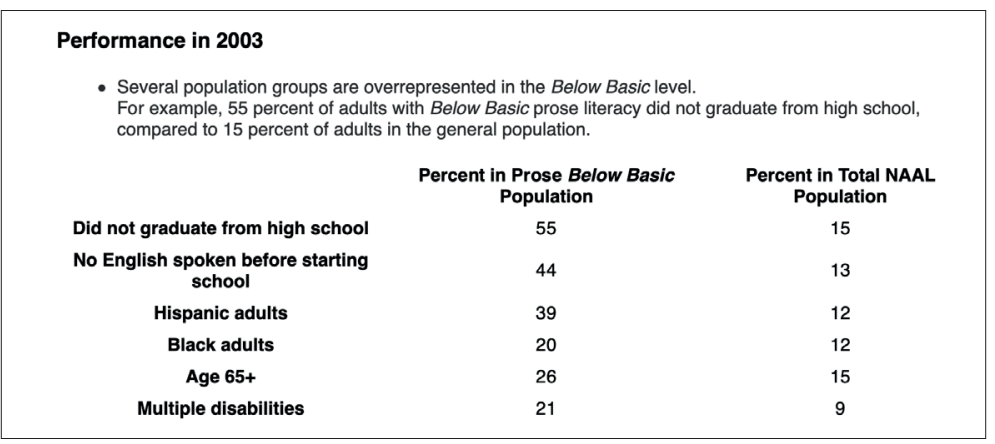

Dalla figura si evince che, fra i detenuti, la percentuale di coloro che non raggiungono i livelli minimi di competenza in literacy e numeracy (below basic level) si attestano ben al di sopra di quelli della popolazione generale nella stessa tipologia di test; i detenuti che non possiedono un titolo di studio sono più del triplo di cittadini del mondo libero con la stessa caratteristica; le competenze linguistiche nella lingua target (l'inglese) sono assenti nel triplo delle persone che hanno sostenuto il test in carcere. Secondo l'UNESCO, anche se è difficile fare una stima precisa, si valuta che almeno un terzo delle persone in carcere presenti dei bassi livelli di alfabetizzazione. Più precisamente, negli USA e in Europa, in media il 25-40\% dei detenuti è funzionalmente analfabeta e, di questi, il 5\% è completamente analfabeta (Clark, Dugdale 2008).

A sostegno di questa tesi è possibile citare The Prison Adult Literacy Survey (Morgan, Kett 2003), lo studio condotto in Irlanda nel 2001 esclusivamente su detenuti e caratterizzato da un impianto metodologico mutuato dai test OCSE. Anche i risultati di questo studio evidenziano disparità di competenze tra cittadini liberi e detenuti; ben il 53\% della popolazione detenuta irlandese si situava sul livello 1 di alfabetizzazione (il più basso nel National Qualifications Frame- 
work) in relazione al 23\% registrato nella popolazione generale. Dai risultati emerge anche un evidente legame tra disagio educativo e comportamenti anti sociali, dal momento che in Irlanda il tasso di criminalità per i maschi è considerevolmente più alto per i giovani che abbandonano la scuola $(46,6 \mathrm{su} 1.000)$ rispetto a coloro che ottengono la licenza (Leaving Certificate, 1,6 su 1.000). Nella stessa direzione vanno i risultati della ricerca condotta da Savoia (2013) in ambito italiano, che evidenziano, nello specifico contesto del carcere di Spoleto, una concomitanza tra successo educativo, sviluppo di migliori abitudini culturali e minor possibilità per i detenuti di cadere nei processi di prigionizzazione e disculturazione.

I dati mettono quindi in luce in maniera inequivocabile il legame tra basse competenze e rischio di finire in un percorso di devianza; nello specifico, per i detenuti stranieri tale rischio è ancora più alto.

Per quanto riguarda i detenuti stranieri in Italia, che spesso sono persone alle quali non è stata riconosciuta la possibilità di ottenere uno status giuridico, dei documenti e conseguentemente hanno intrapreso un percorso ai margini della società, costellato di eventi che le hanno indirizzate all'emarginazione e all'esclusione sociale, la situazione è ancor più drammatica. A titolo d'esempio, nelle classi di alfabetizzazione (387 corsi) gestite dai CPIA nel sistema penitenziario italiano, la presenza straniera rappresenta l'88,5\% degli iscritti, secondo i dati del XIV Rapporto Antigone sulle condizioni di detenzione. In precedenti pubblicazioni (Benucci, Grosso 2015; 2017; Benucci 2018; 2021), infatti, è stato evidenziato come l'istruzione e la possibilità di intraprendere percorsi educativi in carcere, spesso la prima istituzione ad erogare percorsi formativi con la quale i cittadini stranieri vengono a contatto, costituiscano uno dei maggiori incentivi alla riduzione del tasso di recidiva, oltre ad essere raccomandati da numerosi documenti europei, fra i quali:

- il Protocollo nr. 1 alla Convenzione europea per la salvaguardia dei diritti dell'uomo e delle libertà fondamentali (1952), che afferma che «a nessuno deve essere negato il diritto all'istruzione» (art. 2);

- la Carta sociale europea (1996), che stabilisce il diritto al lavoro, il diritto all'orientamento professionale e al diritto alla formazione professionale;

- il Trattato di Lisbona (2009), che ha riconosciuto i diritti dei cittadini dell'UE attraverso l'applicazione della Carta dei diritti fondamentali; l'articolo 14 della Carta riconosce che «tutti hanno il diritto all'istruzione e all'accesso alla formazione professionale e continua».

Il Consiglio d'Europa ha inoltre approvato una serie di raccomandazioni relative in particolare ai detenuti stranieri che gli Stati membri dell'UE si sono impegnati a recepire, in particolare la raccoman- 
dazione del 1990 sull'istruzione in carcere e le norme penitenziarie europee (rivista nel 2006). Secondo il report di sintesi pubblicato nel 2013 Prison Education and training in Europe. Current state of play and challenges (Hawley et al. 2013), ${ }^{14}$ l'educazione in carcere genera infatti una riduzione del costo sociale del tasso di criminalità, grazie alla maggiore capacità di trovare un impiego al termine della pena, favorita dall'abbinamento istruzione/formazione professionale; un maggior senso di appartenenza alla comunità, rafforzamento dei vincoli sociali, alla base della costruzione del cosiddetto 'capitale sociale'. La lotta all'analfabetismo e al rafforzamento delle competenze di literacy e numeracy rappresenta sicuramente il primo passo in questa direzione, in particolare in questo contesto.

Sia nel mondo libero che nel contesto carcerario, l'apprendimento della lingua target in relazione ai diversi livelli di literacy e allo status degli studenti è stato oggetto di molti studi, specie in ambito anglosassone (Klein, Martohardjono, Bigelow, Watson in Browder 2018); la ricerca in questo settore, infatti, si è resa necessaria per chiarire quale sia la relazione tra apprendimento della lingua target e livello di scolarizzazione precedente, variabile estremamente mutevole nei gruppi classe di L2.

Come anticipato in precedenza, infatti, la classe ad abilità differenziate (Caon 2016; Caon, Tonioli 2016) è il contesto principale all'interno del quale vengono inseriti i refugee background students, siano essi neo arrivati nelle società target o persone il cui progetto migratorio si è interrotto drammaticamente o è stato fallimentare.

Ogni classe rappresenta una realtà a sé stante, in cui i fattori menzionati prima e diversificati per ogni individuo giocano un ruolo fondamentale. Uno dei fattori chiave è rappresentato dal livello di scolarizzazione pregresso. Studi recenti, sia di tipo quantitativo che qualitativo (Browder 2018; Ripley Crandall 2018; D’Agostino, Sorce 2016), evidenziano come esista un'enorme variazione nelle competenze possedute dagli studenti cosiddetti SILFE (students with limited or interrupted formal education), variazione che viene correlata a uno sviluppo più o meno difficoltoso della competenza nella lingua target, oggetto di numerosi studi e ricerche finalizzati a descrivere al meglio questa condizione operazionalizzando i diversi aspetti che la compongono, come nel caso dello studio condotto da Browder (2018). Molti studi in letteratura evidenziano la correlazione tra mancanza di competenze alfabetiche in L1 e apprendimento più lento della lingua target, nello specifico dell'inglese come seconda lingua (Browder 2018; Klein, Martohardjono 2015; Tarone, Bigelow 2005).

Come sostiene Caon: 
Occorre quindi integrare le azioni sorvegliando gli stadi dell'interlingua, considerando le diverse culture scolastiche d'appartenenza degli studenti o, come nel caso degli analfabeti, anche la non conoscenza del mondo scolastico (routines, dinamiche relazionali) e la mancanza dello sviluppo delle abilità di lettura e scrittura in L1. $(2019,38)$

È necessario inoltre avvicinare gli studenti agli impliciti culturali della classe intesa come comunità di pratica attraverso spiegazioni esplicite, che chiariscano agli studenti le dinamiche comportamentali, le routine discorsive sia del contesto educativo che del mondo esterno.

Partendo dagli assunti connessi all'Educazione degli adulti, argomento per il quale si rimanda a numerosi ed esaustivi studi ${ }^{15} \mathrm{e}$ al capitolo 2 di questo volume, è fondamentale definire i bisogni comunicativi degli apprendenti, nel quadro dell'inclusione dei migranti in società

altamente testualizzate, dove la scrittura tende ad essere sempre più 'un tramite imperativo nelle relazioni umane' (Adami 2008), le pratiche di alfabetizzazione diventano fondamentali, sia per favorire un processo di acculturazione, che ridefinirà l'intero sistema di riferimento sociale e simbolico dei migranti sia per stimolare un ruolo attivo e consapevole degli stessi all'interno delle società ospiti. (Borri 2019, 90)

I bisogni degli apprendenti analfabeti e a bassa scolarizzazione non erano presenti nella prima versione del Quadro Comune Europeo di riferimento per le Lingue (QCER 2002), limite rilevato precocemente da molti studiosi e docenti. I domini di riferimento per la popolazione immigrata, come l'ambito educativo e l'ambito professionale non erano considerati, c'era scarsa attenzione per la prospettiva di apprendimento da lingue tipologicamente distanti, mancavano descrittori specifici per il livello precedente al pre-A1 (Minuz, Rocca, Borri 2014), dal momento che le competenze in letto-scrittura vengono date per scontate. Nemmeno con l'integrazione del Quadro proposta attraverso la pubblicazione del Companion volume i profili degli apprendenti analfabeti o debolmente alfabetizzati vengono presi in considerazione, nonostante la necessità, sentita in tutti i Paesi europei e di cui si è detto sopra, di elaborare interventi specifici, con contenuti e scopi ‘su misura' per questo profilo di apprendente. A partire da questa esigenza sono stati ideati e implementati per le diverse lingue e per diversi livelli di progressione dei sillabi, partendo dall'acqui-

15 Fra le molte opere sull'argomento si rimanda ai capisaldi: Knowles 1993; Demetrio 1997a; e a una rassegna sui percorsi di formazione degli adulti promossi dall'Università italiana (Federighi 2018). 
sizione delle competenze tecnico-strumentali in letto-scrittura per approdare a una piena competenza funzionale (Minuz, Rocca, Borri 2017). Questa proposta, elaborata da un gruppo di esperti e promosso dalle politiche linguistiche del Consiglio d'Europa nell'ambito del progetto LIAM (Linguistic Integration of Adult Migrants), ${ }^{16}$ ha generato un Quadro comune europeo per l'alfabetizzazione e la L2 - European Framework for L2 and Literacy Teaching (in preparazione).

I sillabi nati da questa operazione e in alcuni casi sperimentati (come nel caso dell'italiano L2) hanno permesso ai docenti di organizzare al meglio i contenuti per l'insegnamento, prestando anche attenzione allo sviluppo delle competenze alfabetiche, e di usufruire di validi strumenti per la didattica ai rifugiati come ad esempio il Toolkit per il supporto linguistico a rifugiati ${ }^{17}$ offerto ai docenti (volontari e non) che operano con richiedenti asilo e rifugiati.

Analizzando questi strumenti, è possibile comprendere come l'aspetto chiave da prendere in considerazione con questo profilo di apprendenti sia la necessità di considerarli come persone che devono non solo acquisire o consolidare delle competenze in letto-scrittura, ma anche e soprattutto apprendere i codici per decodificare in maniera corretta la nuova realtà di inserimento e agire discorsivamente nella nuova comunità. L'aspetto legato alla scelta di materiali adeguati, ad esempio, è centrale poiché determina la possibilità di sensibilizzare gli apprendenti ai diversi codici semiotici: oggetti come una scritta presente in un'insegna, che gli apprendenti possono osservare sia nel contesto in cui si trova collocata realmente (una strada, una piazza) che in una riproduzione cartacea o digitale; presenta poi l'ulteriore vantaggio di collegare l'esperienza diretta, in cui l'apprendente si vede coinvolto in prima persona, all'apprendimento linguistico, aumentando la percezione dell'utilità del corso di alfabetizzazione (Borri 2019; Minuz 2005).

Molti degli approcci all'alfabetizzazione prevedono inoltre il ricorso all'autobiografia (Demetrio 1997b; Ripley Crandall 2018; Montero 2018), o alla scrittura creativa (approccio LEA, Language Experience Approach) abbracciando l'idea che la possibilità di ricorrere alla narrazione della propria storia in classe aiuti a dare senso al processo di inclusione e integrazione nella nuova realtà, secondo una prospettiva etnografica.

Nel Language Experience Approach, ad esempio, i materiali prodotti oralmente vengono poi riusati in forma scritta dall'insegnante per le attività di comprensione e di letto-scrittura. L'uso dell'autobiografia, però, non è un terreno facilmente praticabile, giacché, 
come ricordato prima, i vissuti dei rifugiati possono essere costellati di traumi di vario tipo che non sempre è il caso di chiedere di richiamare alla memoria.

Alcuni altri aspetti centrali nella pianificazione di un corso destinato a rifugiati sono sicuramente la valorizzazione della dimensione metacognitiva (attivare processi di decodifica della società testualizzata in cui hanno vissuto fino a ora, attingere ad esperienze dirette, usare e valorizzare il repertorio linguistico), anche per attivare le strategie di problem solving volte a gestire compiti e richieste funzionali all'inserimento nel contesto sociale (Caon 2019, 41).

Come affermato in precedenza, coloro che non sono mai stati a scuola affrontano sfide che sono molto diverse da coloro che hanno una certa conoscenza di base di cosa siano la lettura e la scrittura. Ciononostante, gli studenti analfabeti hanno delle idee pregresse sull'apprendimento della lingua e sull'alfabetizzazione, idee che provengono dalle esperienze tramandate e comunicate nei propri Paesi di origine. Ė perciò importante promuovere nelle classi di alfabetizzazione delle discussioni preliminari, che gettino le basi per far sentire gli studenti stessi protagonisti del processo di apprendimento.

In un pionieristico volume, Bringing Literacy to Life, Wrigley e Guth (1992) mettono in evidenza come nei programmi innovativi i processi di insegnamento:

- usino l'esperienza dei discenti come punto di partenza per l'alfabetizzazione valorizzando per l'insegnamento le abilità che gli adulti possiedono già, piuttosto che evidenziare gli aspetti deficitari;

- mettano in luce gli aspetti della scrittura come pratica sociale, collegando i contesti in cui la lingua (scritta e orale) è usata e presentando la lingua nelle situazioni comunicative determinate da tali contesti;

- cerchino di indurre gli studenti a scoprire le intenzioni comunicative di un messaggio o di chiarire le proprie intenzioni comunicative;

- sottopongano all'attenzione degli studenti testi interi e presentati in contesto, anziché parole o lettere isolate;

- finalizzino le competenze linguistiche alla trasmissione e alla ricezione dei messaggi, non considerandole fini a sé stesse;

- abbraccino le varie dimensioni dell'alfabetizzazione (personale, sociale, culturale, politica, economica) e sostengano l'idea che l'alfabetizzazione consente la realizzazione di un insieme di funzioni tra le quali risolvere problemi, autopromuoversi, sopravvivere ecc.);

- contemplino l'integrazione tra le quattro abilità;

- promuovano discussioni su temi 'caldi' riguardanti l'integrazione e l'inclusione sociale (ad es., il conflitto tra generazioni che può generarsi nella migrazione; 
- facilitino l'accesso alle informazioni di cui gli studenti hanno bisogno;

- incoraggino gli studenti nell'autonomia per l'accesso alle risorse e per formulare le proprie richieste, anche di supporto e aiuto.

Appare chiaro, da questo esaustivo elenco, che il ruolo del docente in questi contesti va ben oltre la funzione tradizionalmente attribuitagli. Oltre alla necessità di possedere «una solida formazione glottodidattica, consapevolezza delle questioni acquisizionali, sensibilità tipologica» (Arcuri, Mocciaro 2016, 314), il docente deve avere ulteriori competenze che gli permetterano di agire in classe:

- come vero e proprio mediatore, che fornisce ai propri studenti le chiavi d'accesso (linguistiche e non solo) per decodificare il contesto d'arrivo;

- come figura in grado di valorizzare i punti di forza di questi apprendenti;

- come persona in grado di comprendere e gestire stati d'animo ed emozioni scaturiti da traumi e difficoltà psicologiche;

- come guida per preparare gli apprendenti al contatto (non sempre positivo) con il contesto di accoglienza.

Di fronte a una tale pluralità di funzioni da svolgere i docenti potrebbero sentire il bisogno di una guida volta a orientare le attività da proporre in classe per raggiungere di volta in volta i diversi obiettivi elencati sopra, specie in classi multilivello e ad abilità differenziate.

Il primo obiettivo su cui concentrarsi, però, riguarda un aspetto importantissimo della didattica a qualunque profilo di apprendente, ovvero la motivazione. Perché i miei studenti analfabeti/debolmente scolarizzati desiderano intraprendere questo percorso? Cosa ritengono utile imparare?

Sintetizzando quanto emerso da percorsi didattici pregressi in Paesi in cui storicamente si è riscontrata la presenza di questo profilo in contesti di migrazione, come gli Stati Uniti o il Canada (Wrigley, Guth 1992), gli apprendenti hanno evidenziato la necessità di comprendere la cultura e il 'sistema' del Paese target; difendersi da imposizioni di condizioni inique; impostare relazioni proficue e poter reagire, se necessario; relazionarsi con le autorità; spiegare o chiarire dei problemi in situazioni della vita quotidiana; guadagnare autonomia e ottenere riconoscimento sociale, spesso negato a coloro che hanno una competenza parziale della lingua, soprattutto se non sono alfabetizzati; migliorare la propria condizione sociale ed economica; essere in grado di aiutare i propri figli e restituire alla comunità ciò che si è ottenuto in termini di aiuto e supporto. 


\subsection{Bisogni delle classi ad abilità differenziate}

Per implementare quindi in classe una didattica efficace, basata sui bisogni di una classe multilivello con la presenza di studenti analfabeti o scarsamente scolarizzati, è necessario tener presente che il modello operativo da usare dovrebbe essere il modello dell'UdA (Balboni 2014; Brichese 2019), ovvero l'unità di acquisizione o apprendimento, slegata da altre UdA per la caratteristica intrinseca delle classi di migranti adulti, ovvero la presenza oscillante degli studenti. Tenendo presente la stretta connessione tra competenze orali e competenze in letto-scrittura (Tarone, Bigelow 2005; Vinogradov, Bigelow 2010; Brichese 2019), è necessario immaginare attività che promuovano lo sviluppo integrato delle quattro abilità, pianificando momenti in plenaria legati all'oralità e attività differenziate nel momento in cui si passa dalla lettura alla scrittura (Bortolon Guidolin 2019), ricordando che anche i tempi di acquisizione delle competenze orali sono più lunghi per gli studenti a bassa o nulla scolarizzazione.

Uno degli aspetti di cui tener conto nella creazione e sperimentazione in classe delle UdA è relativo alla decodifica delle immagini. Seguendo la struttura tradizionale dell' $\mathrm{UdA},{ }^{18} \mathrm{si}$ è soliti proporre in classe immagini (cartacee o digitali, statiche o in video) che sollecitino lo studente a 'prepararsi' al contenuto che verrà presentato. È quindi necessario ricordare che l'interpretazione delle immagini può non essere immediata per lo studente a bassa scolarizzazione, specie se riproduce realtà dalle quali lo studente è (o è stato fino all'ingresso nel Paese di arrivo) lontano (Altherr Flores 2017). È pur vero che la circolazione di immagini oggi è talmente pervasiva che può essersi costituito un immaginario collettivo di riferimento che travalica i confini geografici, identitari, culturali e linguistici, ma è possibile che immagini relative a specifiche situazioni debbano essere 'chiarite' con l'aiuto del docente. Attraverso un metodo fortemente orientato al significato e alla realtà, nella fase di motivazione e globalità, in cui il docente presenta il materiale, può risultare estremamente utile ancorare la presentazione del lessico, delle situazioni comunicative alla visione di 'oggetti reali' portati in classe dal docente: un pacco di riso, una confezione di detersivo, un portafogli, possono essere portati in classe, osservati, toccati, per passare più agevolmente dalla visualizzazione del segno scritto alla lettura, specie per quegli apprendenti che si avvicinano per la prima volta alla scrittura come sistema di significazione e trasmissione di informazioni e, in generale, per gli studenti che hanno difficoltà di astrazione non avendo sviluppato le specifiche abilità cognitive collegate alle competenze di letto-scrittura.

18 In questo contributo ci limiterà ad alcune considerazioni generali e non si dettaglieranno le tecniche didattiche da utilizzare per ogni fase dell'UdA, per cui si rimanda a Brichese 2019. 
Per quanto riguarda il riconoscimento, riordino e collegamento dei suoni ai grafemi, attività molto utili nella fase di globalità possono essere quelle ispirate al TPR (Total Physical Response): un'applicazione pratica estremamente interessante, tale da essere considerata una buona pratica, è quella proposta da Siebetcheu (2020), che con una squadra di calcio di richiedenti asilo e rifugiati ha sperimentato attività similari, collegando strettamente la formazione linguistica alla principale (e piacevole!) attività svolta in Italia, ovvero il calcio, e usando gli oggetti e gli ambienti dell'allenamento per veicolare contenuti linguistico-comunicativi e nello specifico per favorire il riconoscimento di lettere e sillabe attraverso oggetti e parole familiari ai calciatori (ad es. palloni che riportano parole note scomposte in sillabe e che possono quindi essere riordinati per ottenere la forma corretta della parola). Nella fase di sintesi sono invece da prediligere attività comunicative che prevedano la realizzazione di compiti autentici e basati su simulazioni (ad es. la richiesta di un biglietto dell'autobus, la richiesta di informazioni per strada): quanto prodotto oralmente dagli studenti può essere trascritto e utilizzato dal docente per la fase del lavoro sulla scrittura, secondo il modello operativo proposto da Hokeness (2010). Le parole-obiettivo vengono infatti estrapolate dal testo prodotto dallo studente, e il vocabolo significativo per lui o per lei viene ulteriormente suddiviso nelle unità minime (fonemi o sillabe, o entrambi) sui quali si intende lavorare. Si immagini per esempio che in uno dei dialoghi prodotti nell'ambito di un role play a partire dai già citati realia portati in classe dal docente (ad es., il pacco di riso), recitato a voce alta, venga prodotta la parola 'riso': questa viene letta dal docente sulla scatola del prodotto e segmentata poi nelle unità minime, proponendo attività variegate sulle sillabe o sui fonemi che la compongono. Molto utili possono risultare poi le 'escursioni' in esterna attraverso le quali si può agevolmente creare un collegamento con la realtà (la parola 'riso' verrà a questo punto visualizzata sul cartello di un negozio di alimentari). ${ }^{19}$

È utile a questo punto richiamare quali sono i principali metodi di letto-scrittura ad oggi: la prima grande suddivisione avviene tra metodi globali (o top-down), che partono da parole dotate di senso per arrivare alle singole lettere, sillabe e suoni, e metodi analitici (o bottom-up), che partono dalle unità minime (fonemi o sillabe) per arrivare a unità dotate di senso come parole e frasi. Questi metodi sono sta-

19 L'ulteriore punto di forza delle uscite esterne è costituito dalla possibilità di coinvolgere la popolazione locale nelle attività didattiche (negozianti, operatori di centri per l'impiego), con il risultato di responsabilizzare e rendere consapevoli anche i membri esperti (i parlanti nativi o più competenti nella L2) della comunità di pratica costituita dal contesto di accoglienza, nel processo di alfabetizzazione delle persone a bassa scolarizzazione, processo che non a caso è stato definito all'inizio di questo contributo come pratica sociale collettiva che scaturisce dall'interazione e dalla negoziazione dei significati. 
ti utilizzati tanto per l'alfabetizzazione in L1 che in L2, e, negli ultimi anni, sono stati coniugati nella sperimentazione di metodi integrati, che mutuino aspetti degli uni e degli altri (Condelli et al. 2008). Al di là della scelta del singolo metodo, è importante ricordare che uno degli aspetti maggiormente penalizzanti per gli studenti a bassa scolarizzazione è la mancanza di condivisione degli 'schemi' legati all'enciclopedia personale, quel set di competenze, conoscenze, copioni comunicativi che ci permette di attivare efficacemente l'expectancy grammar per decodificare correttamente un messaggio e mettere in relazione le nuove conoscenze con le conoscenze pregresse (vedi capp. 2 e 3).

Si cercherà adesso di adattare gli elementi chiave per la gestione efficace della CAD individuati dalla scuola veneziana di glottodidattica (Caon 2006; 2008) e presentati nel capitolo 2 per proporre una metodologia didattica in un contesto classe in cui sono presenti analfabeti o studenti a bassa scolarizzazione.

Secondo Caon e Tonioli $(2016,141)$ è necessario tenere in considerazione:

1. «una varietà della didattica all'interno dell'aula» materiali, contenuti e tecniche didattiche;

2. «un'organizzazione flessibile della classe» che rispetti la divisione in gruppi di alunni con lo stesso livello di apprendimento linguistico e quindi eterogenei e che presti attenzione tanto al recupero degli studenti con difficoltà quanto degli studenti eccellenti;

3. «una concezione cooperativa e basata sull'aiuto reciproco tra gli studenti» che collaborando e cooperando nella realizzazione di un compito diventano co-responsabili del lavoro da portare a termine agendo, in questo caso, sulle dinamiche relazionali che si sviluppano all'interno della classe;

4. l'utilizzo di schede di lavoro differenziate per stili di apprendimento e stratificate per complessità del compito (cf. D'Annunzio, Della Puppa 2006) che permettano di agire contemporaneamente su diverse «zone di sviluppo prossimale», secondo la celebre espressione di Vigotskij.

Il primo punto contempla l'aspetto di prioritaria importanza dei materiali didattici. Benché sia noto che i docenti non sempre riescono ad usare e ad adattare ai diversi contesti classe i materiali esistenti, oggi l'editoria propone materiali appositamente studiati per studenti scarsamente scolarizzati o analfabeti, con la premessa che il primo aspetto da considerare sono le aspettative che i docenti nutrono sui propri studenti e su cosa sono in grado di fare.

Molti sono i manuali multilivello che ad oggi possono essere adottati nelle classi ad abilità differenziate e che promuovono gli aspetti proposti nel contributo di Caon e Tonioli. Sia l'editoria nazionale 
che internazionale stanno intervenendo in questa direzione, ${ }^{20}$ pubblicando numerosi testi caratterizzati da:

- presenza di attività pensate per livelli dal Pre-ALFA al B1;

- stratificazione delle attività, con la segnalazione, per ogni attività, del livello dei destinatari per i quali è pensata e specifici consigli per attività destinate agli apprendenti con bassa scolarizzazione;

- presenza di testi autentici e ancorati a bisogni comunicativi degli apprendenti;

- attenzione all'aspetto culturale/interculturale e all'interazione con la comunità ospitante;

- utilizzo di un approccio autobiografico per promuovere l'utilizzo di testi vicini all'esperienza di vita quotidiana dei rifugiati.

Lo strumento Toolkit per il supporto linguistico a rifugiati, pubblicato dal Consiglio d'Europa, è uno dei migliori strumenti al momento disponibili per l'organizzazione interna e la versatilità dei contenuti proposti.

Fra gli strumenti più utili per la gestione della CAD presenti all'interno del Toolkit si segnalano le attività collegate all'uso dei dispositivi mobili e delle nuove tecnologie, per esempio per utilizzare app come Google Maps per l'espansione delle attività didattiche in aula con uscite in esterna, o ancora le app collegate ai manuali didattici, gli strumenti di condivisione di contenuti come Google Drive o altro, come si vedrà nel prossimo paragrafo.

\subsection{Nuove tecnologie a supporto della lotta all'analfabetismo}

In un recente e prezioso contributo, Virgilio $(2018,7)$ ci ricorda che

il quadro politico 'i2010: A European Information Society for Growth and Employment' (Commission of the European Communities 2005) ha definito l'inclusione digitale e la riduzione del digital divide come obiettivi politici strategici dell'UE. Tutti coloro che

20 Per l'italiano sono stati pubblicati, ad esempio il manuale Pari e Dispari (2016, edizioni Loescher); i manuali collegati al progetto ATAYA: ATAYA (2016), ATAYA Prima (2019) corredati dall'app ATAYA (edizioni Sestante). L'utilizzo del materiale multimediale gratuito a disposizione degli studenti permette di ovviare al problema della presenza fluttuante che spesso caratterizza i corsi per migranti; l'uso di video e foto permette di ancorare al contesto le conoscenze linguistiche, sviluppate in parallelo per le 4 abilità. Per lo spagnolo, si segnalano i materiali per classi multilivello: Cuadernos de alfabetización; Manual de Lengua y Cultura (2001, ed. Caritas Española); Contrastes. Método de alfabetización en español como lengua extranjera (1999, ed. MEC); En contacto con... (2000, ed. ASTI); URUK V.3.0; per l'inglese, una rassegna di materiali per classi multilivello, fra i quali ESL Literacy Readers, Whats Next (2011, ed. New Readers Press). 
vivono in Europa, in particolare le persone svantaggiate, dovrebbero poter utilizzare le ICT e beneficiare dell'uso delle stesse per l'accesso ai servizi di base. Il concetto di e-Inclusion (Riga Ministerial Declaration, 2006) definisce l'inclusione digitale sia come uso inclusivo delle ICT, sia come modalità per raggiungere obiettivi più ampi di inclusione sociale.

Molti CPIA, fra cui quello di Pordenone di cui si riporta l'esperienza, hanno focalizzato il lavoro e la riflessione sulla produzione di materiali fruibili a distanza, con l'obiettivo di avvicinare l'esperienza della didattica a studenti con bassa scolarizzazione, sfruttando le nuove tecnologie.

L'uso delle nuove tecnologie nella didattica, testimoniato da diverse esperienze anche in ambito internazionale, ha un potenziale molto ampio per rispondere alle sfide poste dall'insegnamento della L2 a pubblici variegati in classi ad abilità differenziate (Grosso 2020). Da una parte, infatti, esso favorisce un processo di apprendimento personalizzato, avvicinando gli apprendenti alle opportunità educative; dall'altro genera processi di apprendimento sia dell'uso dei diversi codici comunicativi si delle stesse ICT (Codagnone, Kluzer 2011), promuovendo anche la digital literacy. Le ICT sviluppano infatti gli aspetti di interazione sociale e di supporto alla didattica e rispondono a obiettivi didattici specifici (Fiorentino, Cacchione 2011; La Grassa, Troncarelli 2015).

Per i rifugiati, l'uso di app didattiche su smartphone o tablet contribuisce al processo di empowerment e costituisce un'esperienza proattiva di apprendimento basata su reali bisogni e necessità, sfruttando l'abitudine all'uso dei cellulari (Grosso 2021). Attraverso l'uso di questi device è possibile fruire di una facilitazione dei contenuti di apprendimento, con un focus specifico sugli apprendenti analfabeti o debolmente scolarizzati, oggetto di riflessioni e applicazioni in molti progetti europei. ${ }^{21}$

Al centro dell'uso di questi strumenti sta la possibilità di creare un ambiente di apprendimento (learning landscape) contestualizzato e finalizzato allo sviluppo di competenze strettamente connesse allo svolgimento di compiti e interazioni quotidiani e sul lavoro, con i vantaggi legati alla possibilità di fruire dei contenuti sia in autonomia che in un percorso guidato; la possibilità di pianificare lezioni e materiali didattici location-triggered, ovvero generati a partire dagli ambienti reali che frequenteranno i beneficiari, e fruibili dagli apprendenti attraverso l'uso dello smartphone. Il ricorso a questi strumenti, che espande le possibilità del classico percorso didat-

21 Si vedano a titolo d'esempio i progetti MASELTOV, SALSA e Mobile Pedagogy, illustrati in Kukulska-Hulme et al. 2017; o ancora i progetti MALL (Mobile Assisted Language Learning) illustrati in Ahmad et al. 2015 e in Chasikou, Ypsilandis 2019. 
tico guidato, mira al rafforzamento della agency dei rifugiati come protagonisti attivi del percorso di apprendimento che diventa quindi auto-diretto, sociale e caratterizzato da un approccio ludico. Inoltre, nel caso di individui destinatari di resettlement, le nuove tecnologie offrono l'enorme vantaggio di mettere in comunicazione le fasi pre-partenza e post-arrivo, creando dei legami con la comunità ospitante già in fase di pianificazione dell'inserimento. ${ }^{22}$

Per i beneficiari, ci sarebbe la possibilità di instaurare relazioni ad esempio con mentors/tutors/insegnanti - che avrebbero una continuità una volta avvenuto il reinsediamento dei rifugiati nel contesto di arrivo - attraverso l'uso di piattaforme social specificamente collegate ai programmi di formazione linguistica, sia di base sia collegata alle opportunità di lavoro/di formazione professionale (GrünhageMonetti, Braddell 2017, 303). I rifugiati potrebbero infatti avviare, già nel Paese di origine/di primo asilo, il percorso di formazione linguistica che proseguirà poi nella fase post-arrivo, e nello specifico, cominciare a: 1) apprendere in maniera interattiva il lessico e, in forma di chunks, le espressioni comuni utili per i primi mesi nella società di arrivo, obiettivo in linea con i principi della didattica acquisizionale (Vedovelli 2002b; Vedovelli, Villarini 2003; Rastelli 2009); 2) essere esposti a un flusso di input linguistico nella lingua target; 3) fruire di ambienti di apprendimento differenziati con input linguistici graduati e specifici per gli studenti debolmente scolarizzati/analfabeti.

La selezione e lo sviluppo di materiali didattici adeguati ai pubblici di riferimento è al centro di molti saggi e articoli glottodidattici (Cortés Velásquez, Faone, Nuzzo 2017); in relazione agli studenti con bassa scolarizzazione, spesso la scelta del materiale adeguato costituisce il discrimine tra un oggetto reale, tangibile, che segna il contatto con la didattica percepito come accessibile, e invece un elemento che per gli studenti può rappresentare un ostacolo insormontabile (Amoruso 2010). Più in generale, un'ampia riflessione su quali contenuti offrire ai propri studenti e sulle caratteristiche del proprio insegnamento, costituisce un'ottima base per decidere quale materiale può riflettere al meglio la propria visione e andare incontro alle reali esigenze degli apprendenti.

I seguenti esempi sono tratti da progetti o realtà in cui vengono messe in atto strategie di insegnamento che introducono gli allievi alla letto-scrittura, seguendo i principi comuni qui elencati e presentati nel volume pionieristico di Wrigley e Guth (1992).

22 L'ideazione e la realizzazione di progetti che creino un ponte tra la fase pre-partenza e la fase post-arrivo per i beneficiari di resettlement è stata oggetto di una call nell'ambito del programma FAMI; a tale call, l'Organizzazione Internazionale per le Migrazioni ha risposto proponendo il progetto COMMIT, di cui l'Università per Stranieri di Siena è stata partner, che prevede la creazione di attività di connessione tra le due fasi. Per un approfondimento sul progetto si veda: https://eea.iom.int/commit. 
1. Nella quasi totalità, i docenti usano attività di meaning making legate all'attribuzione di significati, con attività di associazione fra immagini e oggetti reali (ad es., un documento di identità reale e una foto di un documento di identità su un testo/materiale didattico), di sviluppo delle capacità cinetiche (tenere in mano una penna/matita, tracciare segni grafici con il dito, anche attraverso l'ausilio di tablet, come nel caso del progetto TABULA ${ }^{23}$ );

2. in molti casi gli studenti e gli insegnanti condividono informazioni sul proprio Paese di origine, portano in classe foto della propria famiglia; la condivisione, all'aumentare delle competenze, può portare alla costruzione di storie, materiale linguistico autobiografico che, come indicato in precedenza, può costituire la base di partenza di un approccio basato sull'esperienza degli apprendenti. In altri casi, gli apprendenti possono situare il proprio viaggio su mappe geografiche e partire da lì per produrre materiale che verrà trascritto e usato dal/dalla docente; è bene tuttavia ricordare che il viaggio è spesso legato ad eventi traumatici e non è quindi opportuno forzare gli studenti a parlarne, ma bisogna limitarsi a seguire eventualmente il filo di un racconto offerto dallo studente. A tal proposito, è possibile lavorare anche sulla conoscenza reciproca tra membri del gruppo chiedendo agli studenti di preparare dei cartelloni sui propri Paesi di origine (ovviamente con l'aiuto del docente) e raggrupparli secondo criteri come la distanza dal Paese target, le lingue comuni o altro);

3. un punto molto controverso e ancora dibattuto nell'ambito della ricerca sulla literacy riguarda la necessità o l'opportunità di alfabetizzare lo studente nella sua L1 prima che nella L2. A partire da Cummins (1979), molti studiosi affermano l'importanza che l'alfabetizzazione in L1 svolge per velocizzare e migliorare l'acquisizione della L2, ma, come afferma Pellitteri (2015):

vista anche la difficoltà di trovare le risorse (mediatori culturali, docenti che conoscono la lingua dello studente ecc.) per procedere in questa direzione, tale idea viene spesso accantonata, anche se va ricordato che la ricerca sta dando a questo aspetto un'importanza sempre maggiore.

4. All'aumentare della competenza linguistica, gli insegnanti propongono, in misura sempre maggiore, attività di ricono-

23 Per una descrizione del progetto si veda: https://epale.ec.europa.eu/it/blog/ tabula-un-tablet-imparare-litaliano. 
scimento di lettere e sillabe (come combinazione di suoni), a partire dai nomi degli studenti o altre parole a loro familiari, come le scritte presenti in prodotti che gli studenti usano, le scritte presenti dentro e fuori dalla scuola ('uscita/entrata', 'pericolo');

5. l'introduzione della scrittura è graduale e prevede la possibilità di usare supporti come strisce di cartone che possono poi essere ritagliate per favorire la segmentazione delle parole. Le produzioni degli studenti, realizzate su supporti digitali o cartacei, vengono conservate ed 'esposte' in classe per incoraggiare e motivare gli studenti, così come le autobiografie (linguistiche o di altro tipo). Vengono promosse attività che favoriscano la creazione di uno spirito di gruppo e legami tra gli studenti, così che la classe diventi un ambiente di scambi significativi, autentici, in cui si comunica anche per il piacere di farlo.

Le attività menzionate promuovono il legame tra le conoscenze pregresse degli adulti e creano fiducia, dando un senso alle attività didattiche che si svolgono in classe; l'approccio didattico al Linguistic Landscape, basato sull'idea di usare i testi esposti come input linguistici autentici in L2, può fornire idee e risorse utilissime per collegare il dentro e il fuori dalla classe, come riportato in esperienze nazionali (Bagna, Gallina, Machetti 2018) e internazionali (Cotto Medina, Freeman in Wrigley, Guth 2000; Malinowski, Maxim, Dubreil 2021) e promuove un approccio alla L2 situato attraverso l'esposizione a testi multimodali, la cui gestione da parte degli apprendenti è sempre più necessaria nelle società contemporanee come affermato all'inizio di questo contributo.

Infine, si ritiene opportuno concludere questo capitolo con una serie di domande, riprese dal già citato volume di Wrigley, Guth (2000) e attualizzate, che possono aiutare i docenti a valutare l'adeguatezza dei materiali didattici in relazione a questo profilo di apprendenti così peculiare. Le domande sono raggruppate in sei sezioni, con l'obiettivo di valutare:

- il collegamento tra i materiali e le aspettative degli studenti;

- la correlazione tra le attività che promuovono la letto-scrittura e le abilità di comprensione e produzione orale;

- la correlazione tra le attività di scrittura e i bisogni comunicativi degli studenti;

- la diversificazione dei materiali e delle attività in relazione alla classe ad abilità differenziate e ai diversi stili presenti, retroterra linguistico-culturali, percorsi di scolarizzazione pregressi;

- il collegamento tra i materiali e la realtà vissuta dagli studenti, la valorizzazione della loro possibilità di esprimersi in maniera creativa e di vedere il frutto del loro sforzo (le produzioni) utile per 
- l'adeguatezza nell'evitare l'etnocentrismo veicolato da stereotipi nella rappresentazione di donne, immigrati ecc.;

- l'adeguatezza nel non dare per scontati elementi culturali e interculturali della società di arrivo.

\section{Lingua e alfabetizzazione}

$1 \quad$ Will students feel both successful and challenged doing the lessons?

Gli studenti si sentiranno allo stesso tempo gratificati e messi alla prova nel partecipare alle lezioni?

2 Do the materials try to link listening and speaking to reading and writing? I materialicercano di collegare l'ascolto e la produzione orale alla letto-scrittura? Do the materials focus on meaningful reading and writing for a purpose (other

3 than practice)? Are there enough opportunities for students to 'try out' writing and express their ideas on paper?

I materiali si focalizzano sulla lettura significativa e sulla scrittura 'controllata' (altra rispetto alla pratica?) Ci sono sufficienti opportunità per gli studenti di provare a scrivere ed esprimere le loro idee su carta?

Do the activities present a good balance between word recognition skills and letter identification on the one hand and whole language activities on the other? Do the activities provide enough opportunities for global processing? Le attività presentano un buon equilibrio tra abilità di riconoscimento di parole e lettere da un lato e attività basate sulla lingua nella sua globalità? Le attività offrono opportunità sufficienti per una processazione globale?

Do the materials take advantage of the print environment that exists in the

5 students' communities? Do they present opportunities to develop print awareness?

Imaterialifanno uso delle scritture esposte che esistono nelle comunità deglistudenti? I materiali presentano opportunità per sviluppare consapevolezza della scrittura?

Are the materials written at the appropriate level in regard to students' overall

6 language proficiency, literacy background, familiarity with cultural concepts, and prior knowledge?

I materiali sono redatti ad un livello appropriato in relazione alla competenza generale degli studenti, al retroterra di alfabetizzazione, alla familiarità con concetticulturali e alle conoscenze pregresse?

Do the materials encourage students to make hypotheses while reading by asking students to guess the meaning of a word using context cues? Do the materials encourage students to take risks in their writing by going through a process of brainstorming with others, writing down ideas, composing several try-out drafts, and focusing on selected aspects of spelling, punctuation, and grammar in the final editing phase?

I materiali incoraggiano a fare ipotesi durante la lettura attraverso domande agli studenti per imparare il significato di una parola usando indizi di contesto? I materiali incoraggiano gli studenti ad assumersi rischi nel processo di scrittura usando il confronto con gli altri, buttando giù le idee, creando molteplici versioni di bozza e focalizzandosi su aspetti selezionati di pronuncia, punteggiatura e grammatica nella fase della composizione finale? 


\section{Linking text to student needs, goals, and experiences}

Associare i testi ai bisogni degli studenti, agli obiettivi e alle esperienze

Do the materials:

I materiali:

1 Contain the kind of activities that respond to student needs and student goals? Contengono di attività che rispondono ai bisogni e agli obiettivi degli studenti?

2 Reflect the students' reality, their roles, lives, and aspirations? Riflettono la realtà degli studenti, i loro ruoli, le loro vite e le loro aspirazioni? Help students to link their own experience to the activities and tasks? Do they 3 provide space for student contributions, such as learner-generated materials, student research, etc.?

Aiutano gli studenti a collegare la loro esperienza alle attività e ai compiti richiesti? Forniscono spazio per i contributi degli studenti, come ad es. materiali creati dagli studenti, ricerche deglistudenti ecc. Provide a link to the community, such as realistically reflecting issues that the community faces?

Forniscono un collegamento con la comunità, ad es. riflettendo realisticamente i problemi che la comunità affronta?

\section{Extending the students' background knowledge}

Ampliare le conoscenze di base degli studenti

Do the materials:

I materiali:

1. Help activate the background knowledge that students bring to class and call on the coping strategies that they have developed?

Aiutano ad attivare le conoscenze di base che gli studenti portano in classe e fanno appello alle strategie di coping che hanno sviluppato? Help students acquire new ideas by linking their experiences and ideas to larger issues in the community or the society?

Aiutano gli studenti ad acquisire nuove idee collegando le loro esperienze e idee a questioni più ampie nella comunità o nella società?

3 Contain opportunities to develop critical thinking and problem solving? Prevedono opportunità per sviluppare il pensiero critico e il problem solving? 


\section{Cultural bias}

Pregiudizi culturali

Do the materials present positive images of immigrant groups in general and of

1 the student group in particular? Do they avoid stereotyping according to age, gender, and ethnic group?

I materiali presentano immagini positive dei gruppi di immigrati in generale e del gruppo di studenti in particolare? Evitano stereotipi in base all'età, al sesso e al gruppo etnico?

Do the materials stereotype by omission? Are women, the elderly, Asians, Latinos, Blacks, and other groups absent or do they appear only as tokens?

I materiali risultano stereotipati per omissione? Le donne, gli anziani, gli asiatici, i latini, i neri e altri gruppi sono assenti o appaiono solo come comparse?

Is there an ethnocentric slant to the way cultural information is presented? Do

3 the materials give the impression (or even insist) that there is one right way of doing things or do they explain options and choices?

C'è un taglio etnocentrico nel modo in cui vengono presentate le informazioni culturali? I materiali danno l'impressione (o addirittura insistono) sul fatto che ci sia un solo modo giusto di fare le cose o spiegano opzioni escelte?

4

Are the contributions of immigrants and minorities in general and the learners' groups in particular adequately represented in the readings?

I contributi degli immigrati e delle minoranze in generale e dei gruppi distudenti in particolare sono adeguatamente rappresentati nelle letture?

Do the materials allow learners to present their own perspectives on life or

5 do they simply show adults in their roles as model workers, model parents, or model community members?

I materiali permettono agli studenti di presentare le loro prospettive di vita o mostrano semplicemente gli adulti nei loro ruoli di lavoratori modello, genitori modello o membri della comunità modello?

\section{Linking teacher experience and interest with the materials}

Collegare l'esperienza e l'interesse dell'insegnante con i materiali

1 Given the background of the teachers, do the materials look 'teachable'? Dato il background degli insegnanti, i materiali sembrano 'insegnabili'?

2 Would the materials be a joy to teach and fun to learn from? What activities can be used to 'lighten up' literacy overload?

I materiali sono gradevoli da usare dal punto di vista di chi insegna e divertentiper chi impara? Quali attività possono essere usate per 'alleggerire' il sovraccarico dialfabetizzazione?

3 Does the content reflect the interests of both teachers and students? Il contenuto riflette gli interessi degli insegnanti e degli studenti?

$4 \quad$ Is it clear how teachers are meant to use these materials? È chiaro in che modo gli insegnanti dovrebbero usare i materiali? 


\section{Adaptability}

Adattabilità

1 Can the materials be adapted to various learning and teaching styles? Imaterialipossono essere adattatia diversistilidiapprendimento insegnamento? Are there enough different kinds of activities so all students can develop a range of skills?

Ci sono tipologie di attività sufficientemente diversificate in modo da far sviluppare aglistudenti un ventaglio di competenze?

3 Do the activities lend themselves to whole group discussions, small group work, pair activities, and individual reading and writing?

Le attività si prestano a discussioni di gruppo plenarie, lavoro in piccoli gruppi, attività a coppie, lettura e scrittura individuale? 


\section{Tecniche e strumenti per la gestione della relazione mentor-rifugiato}

Viola Monaci

Università per Stranieri di Siena, Italia

Sommario 6.1 Processi di integrazione per soggetti vulnerabili. - 6.2 Figure interessate alla mentorship. - 6.3 Strumenti di valutazione e autovalutazione dei programmi di reinsediamento. - 6.4 Analisi dati questionario di autovalutazione.

\subsection{Processi di integrazione per soggetti vulnerabili}

Il processo di migrazione internazionale è un fenomeno articolato che coinvolge diversi elementi (politici, economici, sociali, storici e ambientali), che necessitano di essere presi in considerazione con il fine di conoscere come le società e le manifestazioni migratorie si influenzano, e che recentemente ha acceso un dibattito a livello internazionale. Il primo documento ufficiale ad includere la questione della migrazione è stato l'Agenda 2030 (redatta nel 2015) delle Nazioni Unite, che fra gli obiettivi ha inserito quello delle migrazioni «ordinate, sicure, regolari e responsabili», per mezzo di politiche migratorie pianificate e efficacemente gestite. A questo documento poi si sono aggiunti anche: la Dichiarazione di New York (2016), il Global Compact for Safe, Orderly and Regular Migration (GCM) e il Global Compact Refugees (GCR), entrambi adottati nel 2018. In ottica europea, non esiste ancora un'omogenea divisione degli incarichi nei processi di accoglienza fra gli Stati membri, infatti i documenti europei 
riguardanti politiche e pratiche si rivelano tutt'ora limitanti per migranti e richiedenti asilo negli accessi regolari in Europa, risultando i movimenti migratori affidati al controllo di Paesi terzi che svolgono la funzione di rete prima dell'ingresso nel continente europeo (cf. De Ponte 2018). A tal proposito, nel tempo, sono state sollevate numerose critiche, a partire dai Regolamenti di Dublino (rispettivamente 1997, 2003 e 2014) e la successiva riforma del 2017 proposta dal Parlamento Europeo mai adottata, riguardanti: nel primo caso, il principio del primo Paese di arrivo che comportava una tensione nei Paesi meridionali di primo ingresso; nel secondo caso, invece, il superamento dei criteri di Dublino e la sostituzione del primo Paese di arrivo con un processo meccanico di ricollocamento dei richiedenti asilo, includendo tutti gli Stati dell'UE; infine, la non adeguata protezione ai richiedenti asilo da parte del sistema. Ancora oggi, non è stata attuata alcuna riforma del Sistema comune di asilo in Europa, sulle misure di collaborazione fra Stati membri. Le associazioni del Terzo settore ritengono che non siano sufficientemente valide le politiche adottate dagli Stati e propongono l'adozione di strumenti idonei a garantire corridoi sicuri nella gestione delle migrazioni, ad esempio, come suggerito da Caritas Italiana, i corridoi umanitari sperimentati nel 2015 dalla Chiesa Italiana, e i programmi di resettlement.

A tal riguardo, la comunità internazionale, per garantire la protezione dei rifugiati, ha riconosciuto come politiche tre stabili soluzioni: il rimpatrio volontario, l'integrazione nel Paese di asilo e il reinsediamento (o resettlement). ${ }^{1}$

I progetti di accoglienza, integrazione e inclusione destinati a rifugiati e richiedenti asilo, realizzati in Italia e in altri Paesi membri dell'Unione Europea, vengono quasi esclusivamente gestiti da istituzioni chiamate ad organizzare attività di facilitazione dei processi di integrazione fra le società di residenza e i soggetti considerati vulnerabili: richiedenti asilo, reinsediati, titolari di protezione internazionale ecc. Prendendo in esame la nozione di inclusione, si può affermare che nel ventunesimo secolo questo concetto ha preso forma in rappresentanza delle comunità che si prendono cura dei bisogni di ogni singolo membro senza etichettare o segregare, ponendo enfasi sulla diversità (cf. Bintinger, Eichelberger, Wilhelm 2005), ma comprende anche il significato di valorizzazione dell'individuo e promo-

1 Il rimpatrio volontario prevede il rientro libero dei rifugiati nel Paese di origine solo in circostanze favorevoli di sicurezza. Processo che ha visto una diminuzione dei casi di rimpatrio a causa delle condizioni sfavorevoli per la presenza di conflitti nei Paesi di origine. Per l'integrazione nel Paese di asilo al rifugiato viene concesso il diritto di permanenza, agevolandolo sul diritto all'istruzione, al lavoro, alla libertà di movimento, alla cittadinanza, facilitando quindi il processo di integrazione nella comunità locale. Il reinsediamento (o resettlement) si basa sulla selezione e trasferimento dei rifugiati da un Paese di primo asilo ad un secondo che garantisce loro un'accoglienza permanente. Si tratta di un processo molto articolato in tutte le sue parti e sviluppi. 
zione della partecipazione, dell'uguaglianza, della cooperazione e del dialogo (cf. Schmidt 2017).

I contesti dove avvengono interventi di integrazione richiedono indispensabilmente approcci di tipo inclusivo, sempre più cospicui e caratterizzati da eterogeneità e che si possono definire 'svantaggiati' (Benucci et al. 2021, 17), termine con il quale in questa circostanza si coinvolgono coloro che provengono da un contesto di deprivazione socioculturale come rifugiati e richiedenti asilo. Secondo l'UNHCR (2019), e come anticipato, in questa categoria di 'svantaggiati' sono considerati anche i migranti, nella quale rientrano con diversi appellativi anche altre persone che si trovano costrette a migrare, ossia i rifugiati, i richiedenti asilo, gli sfollati interni, i rimpatriati, gli apolidi, e altre popolazioni in pericolo.

Secondo la sopracitata Agenzia, la Convenzione sullo status dei rifugiati $(1951)^{2}$ chiarisce l'accezione di persona rifugiata in un individuo che, a causa di vari motivi come nazionalità, religione, razza, opinioni politiche o appartenenza a determinati gruppi sociali, può essere perseguitata. Stato questo che la porta a richiedere azioni di protezione da parte di Paesi diversi da quello di appartenenza.

Nel corso degli anni sono nate, e sono ancora in fase di sviluppo, ricerche dedicate all'inclusione sociale di questo particolare pubblico, alcune di esse con focus sulla conoscenza e acquisizione della lingua del Paese di arrivo, in quanto considerata principale strumento di integrazione nella comunità locale. In Italia, infatti, a partire dagli anni Ottanta sono apparse le prime considerazioni sull'insegnamento dell'italiano a stranieri, in prospettiva glottodidattica, con le prime proposte di interventi didattici: materiali didattici, percorsi di programmazione e valutazione delle competenze per immigrati. Fino ad arrivare agli anni Duemila in cui sono emerse le prime riflessioni teorico-applicative sulla formazione dei docenti di italiano L2/ LS per differenti tipologie di pubblico e all'affermarsi del concetto di 'intercultura' legato al plurilinguismo in soggetti migranti (Benucci 2007a; 2008; Balboni 1999; Balboni, Caon 2015).

Si evidenza quindi un'attenzione particolare in contesto italiano nella ricerca sui bisogni educativi di natura conoscitiva e, solo negli ultimi anni, volta all'inclusione didattica di soggetti migranti coinvolti nei sistemi di accoglienza. Pertanto, nel piano strategico Europa $2020,{ }^{3}$ fra gli obiettivi principali, l'Unione Europea aveva fissato anche l'inclusione sociale. All'interno di questo quadro, l'educazione inclusiva, di cui Benucci ha parlato nel capitolo 2 del presente volume, rappresenta lo strumento fondamentale per superare le situazio-

2 Cf. https://www.unhcr.org/it/chi-siamo/la-nostra-storia/la-convenzionesui-rifugiati-del-1951/.

3 Cf. https://ec.europa.eu/info/publications/strategic-plans-2020-2024_it. 
ni di svantaggio e di emarginazione, garantire equità e promuovere i principi democratici della coesione sociale, della cittadinanza attiva e del dialogo interculturale (cf. Read 2020). I documenti europei affermano il ruolo fondamentale della politica linguistica democratica ma l'effettiva applicazione delle raccomandazioni in essi contenuti risulta ancora non soddisfacente sia a livello di osservazione empirica sia in quanto risultante da analisi condotte in vari ambiti e progetti, tra i quali si cita il progetto Include (Majhanovich, Deyrich $2017)^{4}$ che tuttavia evidenzia la presenza di alcune buone pratiche, politiche e risorse completate con successo.

\section{Il Pre-Departure Orientation}

Prima di procedere ad illustrare le caratteristiche dei programmi di Pre-Departure Orientation (PDO), è necessario chiarirne la relativa questione terminologica. 'Orientamento pre-partenzai è una definizione ben consolidata in letteratura per il tipo di attività che verranno descritte in seguito, ma vengono utilizzati anche altri termini, con un significato più o meno ampio: 'fase di assistenza pre-partenza' o 'servizi pre-arrivo' che in generale possono includere pure altri servizi, piuttosto che solamente quelli di cui si parla, anche detti 'programmi di orientamento culturale pre-partenza' o semplicemente 'orientamento culturale' (CO), che è probabilmente il termine predominante in letteratura. Mentre i corsi di orientamento culturale «preparano i rifugiati fornendo informazioni pratiche sul Paese di reinsediamento e li aiutano a stabilire obiettivi realistici e sviluppare le capacità e gli atteggiamenti necessari per riuscire con successo nel loro nuovo ambiente», ${ }^{5}$ prima della partenza dei rifugiati per il Paese di reinsediamento possono essere previsti anche altri servizi come l'addestramento linguistico e l'alfabetizzazione, gli screening sanitari e le valutazioni dello stato di salute, che tuttavia sono spesso separati dai programmi PDO poiché raramente vengono finanziati come parte dell'orientamento culturale. I servizi pre-arrivo o 'misure di sostegno all'integrazione pre-partenza', che non sono necessariamente rivolti solo ai rifugiati da reinsediare, possono anche includere servizi per il lavoro, ad esempio il collegamento di migranti (in genere, lavoratori migranti) con datori di lavoro per trovare un impiego. Ai fini della presente trattazione, però, si focalizzerà l'attenzione sul PDO inteso

4 Progetto finanziato dal programma di apprendimento permanente dell'UE «Lingue, progetto multilaterale. Attività chiave 2» (2007-13), con una partnership di sei organizzazioni europee che si prefiggeva di costruire una rete per lo scambio e la diffusione di linee guida comuni e buone pratiche di politiche linguistiche per la promozione dell'apprendimento delle lingue e della diversità linguistica.

5 Cf. https://www.resettlement.eu/journey/pre-departure-assistance-phase. 
come programma di orientamento culturale. Ma prima, è interessante aprire una piccola parentesi sui programmi PDO attuati negli Stati Uniti e in Olanda, dove è stato scelto di concentrarsi sull'inizio del processo di integrazione già nel Paese di primo asilo, preparando i rifugiati alla società di arrivo attraverso la formazione nella lingua target, e come avviene in Canada e nel Regno Unito, dove l'interesse è stato convogliato nella ricerca attiva del lavoro. In sostanza, ciascuna tipologia di programma conferisce un peso diverso ad ogni obiettivo con l'ausilio di mezzi differenti. La formazione linguistica, per esempio, viene vista come un elemento imprescindibile per i training e la sua progettazione per il percorso didattico, e prevede la definizione di un curriculo comprendente determinati criteri, quali:

- identificazione dei bisogni di uno specifico gruppo di beneficiari;

- descrizione delle finalità e degli obiettivi necessari per soddisfare specifici bisogni;

- descrizione delle finalità e degli obiettivi necessari per soddisfare specifici bisogni;

- elaborazione di un sillabo ${ }^{6}$ appropriato e il relativo metodo di insegnamento, materiali didattici che verranno adottati (cf. Spinelli, Parizzi 2010, 99).

All'interno dei più ampi processi di resettlement, che come sopra citato possono essere definiti come percorsi sicuri adottati dall'Unione Europea per la migrazione di rifugiati, si possono trovare specifici programmi dedicati alla figura del rifugiato reinsediato, sessioni di orientamento pre-partenza (PDO), coordinati e diretti da organizzazioni internazionali come l'OIM, e talvolta con la collaborazione dei Paesi di arrivo, con lo scopo di preparare attraverso azioni di sensibilizzazione e di formazione coloro che si troveranno a doversi integrare nel nuovo Paese di accoglienza, oltre a ricostruire la fiducia dei rifugiati, ad aumentare il senso di controllo sulla propria vita e a fornire loro strumenti per la gestione generale della vita nel Paese di arrivo e in particolare delle situazioni non familiari nelle quali potrebbero trovarsi (cf. Grosso 2021, 28). Secondo quanto dichiarato dall'OIM:

L'integrazione può essere intesa come un processo bilaterale che si verifica in un continuum: il processo di integrazione inizia molto prima che un rifugiato lasci il suo Paese di primo asilo, e continua ben oltre il suo arrivo nel Paese di reinsediamento, mentre crea nuove connessioni e inizia a vivere nella nuova comunità. (2019a, 28)

6 Il sillabo organizza i contenuti linguistici, culturali, pragmatici, sociolinguistici da proporre agli apprendenti, rappresentando uno strumento per organizzare le mete dell'Educazione Linguistica e gli obiettivi di insegnamento/apprendimento, passando dal piano teorico a quello pratico (cf. Benucci 2014, 55). 
E importante sottolineare che queste attività possono essere attivate già nel Paese di origine o di primo insediamento, quindi a distanza, per agevolare la connessione fra due fasi distinte: quella pre-partenza e quella post-arrivo. Le sessioni di orientamento pre-partenza mirano quindi ad affrontare le questioni legate all'integrazione nella fase più precoce possibile del processo di reinsediamento dei migranti.

L'obiettivo principale di fornire servizi di insediamento prima dell'arrivo è quello di accelerare ulteriormente l'insediamento e l'integrazione, poiché si prevede che accedendo a questi servizi i nuovi arrivati diventeranno più informati sul processo di insediamento ed integrazione in una fase precedente e prenderanno iniziative per prepararsi meglio per il loro successivo insediamento ed integrazione. (IRCC 2018, 27)

Entrando più nello specifico dei PDO, si definisce lo scopo primario delle sessioni che nascono per essere di natura informativa: condividere informazioni accurate e obiettive, per garantire che i rifugiati siano ben informati sulle fasi del processo, del viaggio di reinsediamento, e sul Paese di accoglienza (diritti e doveri, usi e costumi, sistema d'istruzione e sanitario, occupazione, abitazioni, gestione del denaro, e altre informazioni di base sul Paese come il clima, la geografia ecc.), nonché informazioni molto pratiche, con il fine sia di fornire aspettative realistiche e non distorte sulle nuove condizioni di vita nel Paese o società di reinsediamento, sia di facilitare la loro integrazione/inclusione. Studi recenti come quelli condotti da Fratzke e Kainz (2019) propongono un modello composto da tre aspetti principali per la definizione dei contenuti della formazione PDO:

1. conoscenze necessarie a gestire il processo di resettlement $\mathrm{e}$ la vita quotidiana nel Paese di destinazione;

2. abilità per affrontare il processo di resettlement proficuamente;

3. attitudini, mentalità e aspettative realistiche per un processo di integrazione positivo.

Affrontare tali contenuti concretamente in sé non è tuttavia l'obiettivo finale del PDO, poiché lo scopo generale, come suggerito dall'OIM (2019a, 34) è quello di preparare i rifugiati aiutandoli a sviluppare le capacità e gli atteggiamenti di cui avranno bisogno per farcela nel loro nuovo ambiente.

La migrazione, ed in particolare il resettlement, possono rappresentare una svolta nella vita dei rifugiati, che beneficiano di nuove opportunità lavorative o di istruzione ma che, allo stesso tempo, devono adattarsi alle nuove richieste dal punto di vista amministrativo-burocratico e a nuove situazioni e modalità di relazione sociale, imparando e usando una nuova lingua. (Grosso 2021, 27) 
Programmare questi interventi di formazione è particolarmente importante in quanto rispetto ai nuovi Paesi di reinsediamento sono poche le informazioni disponibili per i rifugiati, che spesso fanno riferimento a ciò che viene trasmesso loro all'interno delle reti personali su cui molti fanno affidamento per ottenere informazioni che, peraltro, non risultano sempre accurate. È questo il motivo per cui le sessioni PDO riguardano anche «il benessere psicologico dei rifugiati, tenendo conto degli aspetti sociali, antropologici, culturali e psicologici del reinsediamento» (IOM 2019a, 34).

Avere la possibilità di partecipare a queste sessioni di orientamento prima della partenza ha inoltre lo scopo di ridurre lo stress e l'ansia che possono trovarsi a provare i rifugiati nelle prime settimane di vita nel nuovo Paese, e il rischio di un sentimento di delusione, nell'interesse sia dei rifugiati reinsediati che delle comunità e delle autorità che li accolgono. Ulteriore aspetto che può rivelarsi stressante per i beneficiari è l'impossibilità di scegliere il luogo di arrivo nel quale iniziare la nuova vita, e quindi pur aderendo volontariamente al programma si possono sentire sovrastati da un senso di frustrazione e non gestione delle proprie scelte di vita (cf. Grosso 2021). Inoltre, guardando più nel profondo di questi programmi si può affermare che le sessioni vengono pianificate nel dettaglio, infatti, già negli ultimi anni sono stati sviluppati programmi PDO specifici per target di beneficiari vulnerabili o gruppi con bisogni specifici come donne rifugiate, bambini e adolescenti, pertanto «l'orientamento deve affrontare le reali preoccupazioni dei partecipanti ed enfatizzare l'adattamento culturale, la comunicazione intergenerazionale, i ruoli di genere, il cambiamento delle dinamiche familiari e altre sfide» (IOM 2019a, 34). Per riassumere, si riportano di seguito i cinque obiettivi primari della PDO identificati dall'OIM $(2018,3)$ :

1. fornire ai rifugiati informazioni accurate sulla vita nel Paese di destinazione e sul processo di reinsediamento;

2. aiutare i rifugiati a sviluppare aspettative realistiche sul reinsediamento;

3. facilitare i rifugiati a sviluppare la consapevolezza e le competenze necessarie per un adattamento efficace per integrarsi nella nuova società;

4. affrontare e ridurre le preoccupazioni e le domande dei rifugiati;

5. favorire l'empowerment delle donne rifugiate e di altri gruppi vulnerabili di beneficiari.

La conferma del reale valore delle attività PDO, che hanno avuto un crescente consenso, è ben dimostrata dal numero di Paesi di reinsediamento che hanno aderito alle sessioni di orientamento pre-partenza: 19 su 25 Paesi con operazioni di reinsediamento in corso, nel 
2018, hanno realizzato qualche tipologia di corsi di orientamento prepartenza (cf. IOM 2019a, 38-40).

A tal proposito, è considerevole mettere in evidenza che, dal 2015, i Paesi dell'Unione Europea hanno lanciato i programmi di protezione internazionale per rifugiati e richiedenti asilo, che sono stati inizialmente gestiti a livello nazionale per una condizione equa delle responsabilità, e dal 2016, la Commissione Europea ha presentato il Union Resettlement Framework, ${ }^{7}$ un quadro comune per le azioni di reinsediamento, che definisce: il numero dei beneficiari da reinsediare, i Paesi di origine dei rifugiati che aderiscono al programma e i Paesi membri nei quali saranno accolti. Pertanto, tale proposta ha l'obiettivo di fornire fra i Paesi dell'UE un quadro più strutturato, armonizzato e permanente per il reinsediamento e di coinvolgere nel programma una soglia minima di 50.000 beneficiari richiedenti protezione internazionale.

L'importanza della formazione PDO è stata dimostrata da diversi casi studio che hanno messo in evidenza non solo l'efficacia per una migliore integrazione a lungo termine dei beneficiari rifugiati nelle società di accoglienza, ma anche la forte richiesta di partecipazione dagli stessi rifugiati. ${ }^{8}$ Questo aspetto risulta particolarmente significativo se le attività di formazione pre-partenza seguissero l'iter stabilito, ossia il collegamento con le attività post-arrivo finalizzate a ottimizzare l'integrazione e garantire l'inclusione sociale dei rifugiati (uno degli obiettivi cardine del progetto COMMIT). ${ }^{9}$ Un altro aspetto rilevante dei programmi di orientamento emerge dalla presenza di un numero consistente di manuali e linee guida che descrivono le fasi di progettazione e conduzione delle attività di PDO, attraverso l'analisi delle migliori pratiche segnalate da precedenti programmi di reinsediamento, grazie all'osservazione di report e altre pubblicazioni, di testimonianze di autori, organizzazioni e istituzioni, della condivisione di esperienze, aspetti critici e suggerimenti per il futuro, che descrivono il design interno e la metodologia di gestione delle PDO. Per contro, un numero limitato di fonti primarie, corrispondenti a report che descrivono i processi di valutazione condotti per valutare programmi di PDO, è disponibile pubblicamente, a livello internazionale. Ciò è dovuto a vari fattori, come il fatto che «molte valutazioni sono rivolte prevalentemente al pubblico nazionale» (González Garibay, De Cuyper 2013, 87), e il fatto che alcuni stati di reinsediamento usano ancora metodi informali per monitorare e va-

7 Cf. https://www.europarl.europa.eu/legislative-train/theme-towards-anew-policy-on-migration/file-jd-eu-resettlement-framework.

8 Per una rassegna esaustiva sull'argomento si veda Fratzke, Kainz 2019, 3-6.

9 Cf. https://italy.iom.int/it/https\%3A//italy.iom.int/it/aree-di-attivit\% 25C3\%25A๑/relocation-e-resettlement/COMMIT. 
lutare l'orientamento (cf. Fratzke, Kainz 2019, 31). Tali lavori, tuttavia, sono una fondamentale fonte di informazioni sulla metodologia di ricerca da applicare nella valutazione del PDO.

Nel paragrafo 6.3 si cercherà di sintetizzare gli aspetti più interessanti evidenziati da entrambe queste categorie di fonti che è bene prendere in considerazione ai fini della valutazione.

\section{Il Post-Arrival Orientation}

L'organizzazione OIM definisce l'integrazione come un continuum, e specifica che ha luogo attraverso più dimensioni contestuali, «inizia prima che i migranti mettano piede nel Paese di accoglienza e può estendersi ben oltre le fasi iniziali del reinsediamento» (IOM 2019a, 34). L'orientamento pre-partenza, come anticipato nel paragrafo precedente, inaugura il processo di integrazione, fornendo avviamento pratico, informativo e psicologico ai rifugiati oggetto di resettlement, per accrescere la loro autostima, consapevolezza e delucidazioni, oltre a ottimizzare il loro adattamento nel Paese di reinsediamento. I corsi di formazione pre-partenza, tuttavia, non sono sufficienti a garantire il successo del programma di reinsediamento in termini di integrazione/inclusione sociale, né a garantire che gli aspetti pratici delle sessioni siano stati correttamente memorizzati. Come evidenziato dall'UNHCR $(2002,148)$ non esiste un modo per comprendere con certezza il grado di apprendimento e fino a che punto i beneficiari riescono ad immagazzinare tutte le informazioni che gli vengono fornite prima della loro partenza. Per esempio, «l'esperienza dei programmi pre-partenza svedesi suggerisce che potrebbe essere piuttosto difficile per le persone assimilare informazioni riguardanti un Paese profondamente diverso senza averne prima fatto esperienza» (UNHCR 2002, 148). Ecco perché «autorità locali, ONG e altre organizzazioni che lavorano con i rifugiati nei Paesi di reinsediamento continuano questo processo di orientamento nelle comunità locali dopo l'arrivo dei rifugiati» (ICMC 2012, 4).

Alla luce di quest'ultima affermazione, si specifica che le fasi postarrivo e di integrazione si riferiscono al periodo che segue l'arrivo fisico dei rifugiati nei Paesi di reinsediamento. Oltre all'accoglienza iniziale e ad un sostegno a breve termine nella fase di inserimento per il soddisfacimento di bisogni immediati, un supporto a lungo termine viene solitamente fornito fino a dodici mesi dopo il loro arrivo. Inoltre, in questo arco temporale sono previste anche altre sessioni di orientamento culturale e misure di integrazione per i rifugiati, ${ }^{10}$

10 Alcuni programmi di integrazione hanno eccezionalmente una durata maggiore. Per esempio, nel 2008-09, il progetto pilota di mentorship Women on the Move, condotto nell'area di Melbourne, era specificatamente indirizzato a donne in situazioni di iso- 
volte anch'esse al raggiungimento del successo finale, sia per i rifugiati sia per le comunità di accoglienza. È importante, per esempio, garantire la trasmissione dei messaggi che veicolano le sessioni PDO anche nelle sessioni di orientamento post-arrivo, «garantendo [così] un continuum nel fornire informazioni» (IOM 2019a, 29).

Creare un collegamento tra le varie fasi di integrazione attraverso l'allineamento delle attività e un migliore coordinamento tra il supporto pre-partenza e quello post-arrivo favorisce la fiducia ed i risultati di integrazione nel lungo termine. (IOM 2019a, 34)

A conferma di quanto detto, si fa presente che tra gennaio 2018 e luglio 2019, è stato condotto l'innovativo progetto LINK IT con l'obiettivo finale di «rafforzare il legame tra il sostegno all'integrazione pre-partenza e post-arrivo per i rifugiati». ${ }^{11}$ Sta crescendo quindi il consenso riguardo al «riconoscimento che le misure di supporto all'integrazione rese disponibili ai migranti all'arrivo hanno maggiore probabilità di essere efficaci se continuano un processo di integrazione iniziato prima dell'arrivo» (IOM 2019a, 35). La diffusione di queste buone pratiche consiste nel consentire allo staff nei Paesi di reinsediamento di coordinarsi con coloro che si occupano dei programmi di orientamento pre-partenza, condividendo informazioni dettagliate e le caratteristiche specifiche della popolazione di rifugiati, il loro bagaglio personale, le esperienze pregresse, le competenze, il profilo psicologico, i valori e la cultura. Tutti dettagli che però a lungo termine possono aiutare ad «adattare queste informazioni alle esigenze dei fornitori di servizi post-arrivo nel supportare la loro pianificazione del sostegno all'integrazione» (IOM 2019a, 35).

\section{Specificità dei programmi di mentorship post-arrivo}

I servizi offerti ai rifugiati reinsediati, come ad altre tipologie di migranti, nel Paese di reinsediamento comprendono varie misure di supporto: lezioni di lingua, orientamento sociale e culturale, formazione professionale e assistenza per l'accesso ai principali servizi, come quello sanitario e dell'istruzione. Sono numerosi gli attori coinvolti nel processo di integrazione, in quanto inteso come un processo che ha luogo a livello individuale, locale e nazionale, fra i quali si trovano: autorità locali, fornitori di servizi sociali, ONG ecc. Essendo

lamento sociale e con difficoltà di orientamento in corso dopo molti anni di residenza in Australia. Per una rassegna esaustiva dell'argomento cf. Bond 2010.

11 Maggiori dettagli circa il progetto LINK IT sono disponibili al seguente link: https://www.resettlement.eu/page/link-it. 


\section{6 - Tecniche e strumenti per la gestione della relazione mentor-rifugiato}

queste organizzazioni della società civile che supportano i rifugiati reinsediati, sono particolarmente attive nel reclutamento di cittadini volontari che fungono da mentors. I vari ruoli ricoperti dai mentors nell'integrazione dei rifugiati dipendono dal tipo di programma di mentorship a cui partecipano. Alcuni di questi hanno obiettivi specifici, come ad esempio l'apprendimento linguistico, ${ }^{12}$ il tutoraggio professionale ${ }^{13}$ e il supporto per la ricerca di un alloggio. ${ }^{14}$ Sistemi di mentorship specifici sono riservati a sottogruppi vulnerabili della popolazione rifugiata come giovani e donne..$^{15}$ Altri programmi ancora forniscono un'assistenza generale, gestendo bisogni più ampi e prevedendo qualche forma di attività di advocacy, per migliorare l'opinione pubblica e le politiche sull'immigrazione. ${ }^{16}$

La mentorship è un'esperienza fra il mentor e il mentee, che dal primo incontro chiariscono gli obiettivi del programma di lavoro. Si tratta nel dettaglio di un percorso di trasferimento di conoscenza e metodologia in termini di soft skills. Un rapporto che si instaura in un contesto formale, accademico, ma che poi diviene informale con la conoscenza reciproca. Si trasferiscono conoscenza e informazioni, basandosi su fiducia, rispetto e comprensione.

Pur non disponendo di specifiche linee guida su tali processi, gli enti che se ne occupano riescono ad ottenere risultati soddisfacenti, avvalendosi della figura del cittadino volontario, che si trova ad affiancare gli operatori professionalmente formati in tale lavoro. I vo-

12 Per esempio, in Irlanda, il programma Failte Isteach (Benvenuti) pilotato nel 2006 e lanciato a livello nazionale nel 2008 è basato sulla relazione di mentorship tra volontari più anziani e mentees, che frequentavano lezioni di conversazione di inglese. Per maggiori informazioni, cf. https://ec.europa.eu/migrant-integration/intpract/ welcome---failte-isteach?lang=en.

13 Per esempio, Singa Professional Mentoring è un programma condotto in diversi Paesi che mette in contatto i nuovi arrivati e professionisti locali attivi negli stessi campi attraverso una relazione di tutoraggio per alcuni mesi. Sul programma Singa Professional Mentoring in Germania, cf. NiCER Project - Nuovi approcci per l'integrazione culturale dei giovani rifugiati (N.D.), https://nicerproject.eu/wp-content/uploads/Good-practices-IT.pdf.

14 Per esempio, nel 2015 la Caritas belga ha sviluppato un progetto chiamato Housing cafés, dove professionisti e volontari cercano di aiutare rifugiati a cui sia stato appena riconosciuto lo status di rifugiato o la protezione sussidiaria nella loro ricerca di alloggio. Per maggiori informazioni, cf. https://www.caritas.eu/housing-cafe-empowerment-refugees-search-housing/.

15 Per esempio, il progetto WOMENTO della Family Federation of Finland, iniziato a gennaio 2011, è specificatamente destinato a donne immigrate istruite e si pone l'obiettivo di migliorare la loro integrazione nella società finlandese attraverso un sistema di mentorship. Cf. https://ec.europa.eu/migrant-integration/integrationpractice/womento_en?lang=fr.

16 Per esempio, all'interno del Gateway Protection Programme per rifugiati, stabilito nel 2004 nel Regno Unito, «i volontari vanno anche nelle scuole e negli spazi della comunità per parlare dei rifugiati e del reinsediamento, spesso accompagnati da rifugiati precedentemente reinsediati» (ICMC 2012, 26). 
lontari selezionati sulla base di esperienze personali, competenze e reti sociali, vengono definiti quindi mentors.

Nel paragrafo 6.4 di questo capitolo si illustreranno la proposta di intervento e più nello specifico le peculiarità dello strumento di autovalutazione per mentors, frutto di uno studio condotto dal gruppo di ricerca coordinato da Antonella Benucci dell'Università per Stranieri di Siena in tale ambito. Si analizzeranno altresì i principali risultati ottenuti in contesto italiano e i vantaggi dell'utilizzo di tale strumento per l'automonitoraggio in ottica migratoria.

\subsection{Figure interessate alla mentorship}

L'Europa è divenuta nel tempo il continente che ospita il maggior numero di soggetti svantaggiati, come definito sopra, ventaglio che si è ampliato includendo anche la categoria dei rifugiati e richiedenti asilo. Individui provenienti da Paesi colpiti da guerre civili che vivono in uno stato di povertà e con limitate risorse, e che incontrano difficoltà negli Stati membri, causa i sistemi di accoglienza disarticolati o inadeguati rispetto alle loro esigenze.

L'esponenziale crescita di arrivi di richiedenti asilo, rifugiati e minori stranieri non accompagnati in Europa, e nello specifico in Italia, ha variato la tipologia di interventi necessari, sia dal punto di vista numerico (per le persone da accogliere), sia per le diversità tipologiche delle situazioni da affrontare.

La non definizione di politiche migratorie e di integrazione, che rispettino i loro bisogni, priva i sistemi di accoglienza di strumenti inclusivi per soggetti che richiedono di essere integrati nel tessuto sociale di arrivo.

La sfida moderna, quindi, consiste, in un approccio olistico e completo rivolto all'educazione dei rifugiati che includa la politica educativa, le strutture scolastiche, la pratica in classe, i programmi di studio, la pedagogia, i materiali didattici e la consapevolezza culturale della società. (Benucci 2021, 17)

Strumento efficace, ma ancora in via di sviluppo, come chiarito nel paragrafo precedente, è quello della mentorship, una relazione che si instaura fra il mentor e i rifugiati (mentee) con i quali viene messo in contatto. Per chiarire queste due figure, si tiene a specificare che per mentee si intende la famiglia o il membro di una famiglia rifugiata coinvolti nel processo di mentorship. I mentors invece, sono cittadini della comunità di arrivo che supportano le famiglie rifugiate nell'inserimento della società di accoglienza e che quindi diventano una vera e propria risorsa e uno strumento di cooperazione fra le parti. Persone delle comunità locali, compresi coloro che sono sta- 
ti rifugiati reinsediati attraverso programmi precedenti, quindi biculturali e bilingui, che hanno un ruolo di ausilio per i rifugiati in prospettiva della nuova vita nella nuova società «colmando le lacune nell'offerta di servizi per l'integrazione e/o estendendo il tipo e la natura di supporto all'integrazione offerto ai rifugiati» (ICMC 2015, 9).

Una delle maggiori difficoltà dei programmi di mentorship è quella di stabilire dei confini al tipo di assistenza fornita dai mentors ai mentees. Questo perché i mentors ricoprono un ruolo che tradizionalmente è affidato allo Stato o ad altre autorità e contribuiscono a costruire un ponte fra i rifugiati e le comunità locali; forniscono il loro supporto informale e personalizzato per l'accesso ai servizi; assistono i rifugiati nelle sfide quotidiane che possono incontrare; danno loro ascolto; contribuiscono a sviluppare la comprensione della cultura e dei valori della società ospitante; favoriscono contatti sociali, ma possono a loro volta rimandare ad altri fornitori di servizi. La sfida è quella di evitare l'isolamento sociale e renderli attivi all'interno delle comunità locali che si trovano ad affrontare un rapido cambiamento, catapultati all'interno della nuova comunità. Pertanto, da un lato «i volontari giocano un ruolo aggiuntivo fondamentale nel favorire l'accoglienza, l'adattamento e l'insediamento a lungo termine, e nel promuovere la costruzione di amicizie e connessioni nei nuovi dintorni» (ICMC 2015, 4). Dall'altro lato però, il lavoro può semplificarsi se essi stessi sono ex rifugiati reinsediati, e quindi possono rappresentare «una preziosa risorsa di formazione e supporto, sia per i nuovi arrivati, sia per le organizzazioni e i servizi che si occupano di loro» (ICMC 2015, 4). Altro aspetto importante che può rappresentare un vantaggio, è che permettono anche alle comunità locali di «comprendere meglio altre culture e l'esperienza del reinsediamento e favorire l'interazione interculturale» (Bond 2010, 5). Da queste riflessioni ne consegue che il mentoring va inteso come una «relazione di mutuo beneficio» (Bond 2010, 5), sia per i mentees che per le comunità ospitanti.

I mentors, e tutti gli operatori coinvolti nelle relazioni con i migranti, si trovano a gestire situazioni, in ottica interlinguistica e interculturale, facilmente fallimentari e che quindi hanno conseguenze negative sul percorso di integrazione del beneficiario, ma anche sul piano motivazionale e professionale degli operatori, a causa dell'assenza di percorsi formativi specifici (Benucci 2018; 2014; 2013). Proprio per questo carattere fallimentare, si tratta di un fenomeno che si ripercuote nel sistema formativo e che assume una rilevanza specifica nella costruzione di percorsi di integrazione, pertanto è sempre più urgente e necessario formare chi accoglie e supporta i migranti (Damiani, Agrusti 2019).

In riferimento proprio all'esigenza di una specifica formazione, sia teorica che pratica, sarebbe auspicabile avere creatività e flessibilità nelle strategie e nei metodi di insegnamento adottati, così co- 
me negli approcci inclusivi interlinguistici e interculturali a sostegno dell'obiettivo del percorso, l'integrazione sociale. Aspetti questi che presentano un notevole grado di complessità, ma rappresentano una sfida condivisa.

Una grande attenzione è stata rivolta all'uso delle ICT (o TIC, Tecnologie dell'informazione e della comunicazione) $)^{17}$ nella formazione di rifugiati e richiedenti asilo in ragione dell'elevata efficacia dovuta all'aspetto multidimensionale della didattica digitale che permette la coesistenza di più sfere dell'apprendimento (sapere, saper fare ecc.), sia per la facilità di accesso ad altri contenuti utili all'integrazione (siti web delle amministrazioni pubbliche, di servizi di pubblica utilità ecc.) sia allo scopo di incrementare il livello di inclusione e di cittadinanza (cf. Benucci et al. 2021, 19).

Fra tutti i progetti di ricerca-azione, alcuni dei più rilevanti hanno messo in risalto punti di forza specifici dei processi di integrazione/inclusione di rifugiati e richiedenti asilo, fra questi il rapporto con le strutture educative del Paese ospitante: interventi di orientamento alle scelte scolastiche in relazione alle aspettative degli studenti con cittadinanza non italiana e il rapporto di queste con temi quali la valorizzazione del plurilinguismo, la formazione interculturale degli insegnanti e degli operatori e le pratiche didattiche volte alla promozione del dialogo interculturale (Catarci, Fiorucci 2014).

\subsection{Strumenti di valutazione e autovalutazione dei programmi di reinsediamento}

La valutazione rappresenta una fase importante nello sviluppo di un qualsiasi progetto. Ormai da tempo l'interesse verso la valutazione ha avuto un crescente sviluppo, in primis grazie all'introduzione di alcuni approcci metodologici come:

- Results-Based Management (RBM), introdotto fra la fine degli anni Novanta del secolo scorso e i primi anni Duemila, è uno strumento che mira a promuovere una cultura del managing-forresults, un approccio basato sui risultati per il miglioramento dell'efficacia gestionale e delle responsabilità attraverso il monitoraggio dei progressi, e che quindi ha come obiettivo quello di «fornire un coerente quadro per la pianificazione strategica e gestione basata sull'apprendimento e responsabilità in un ambiente decentralizzato»; ${ }^{18}$

- Managing for Development Results (MfDR), un approccio che ha contribuito alla promozione di una cultura della valutazione, e

17 Per una rassegna dettagliata si veda il capitolo 2 di Benucci del presente volume.

18 http://web.undp.org/evaluation/documents/RBMConceptsMethodgyjuly2002.pdf. 
che si basa sull'efficacia dello sviluppo prestando attenzione su un più ampio livello di azione, regionale, nazionale e globale, anziché limitarsi al livello interno di una sola organizzazione; ${ }^{19}$

- Monitoring and Evaluation (M\&E), approccio molto ricorrente in guide e manuali sui processi di pianificazione e gestione di un programma; ${ }^{20}$

La valutazione, infatti, è un processo che ha una forte influenza sulle fasi di un intero ciclo progettuale. Per questo motivo, «un chiaro design del progetto è alla base di un processo di monitoraggio e valutazione di successo» (IOM 2019b, 175), per quanto «una buona pianificazione e un buon design non garantiscono risultati» (UNDP 2009, 82). Inoltre, sia interventi di monitoraggio che di valutazione richiedono alla base una chiara pianificazione, pertanto è necessario sviluppare dei modelli di M\&E già al principio dell'organizzazione di un programma o di un progetto considerandoli parte integrante della gestione di questi come sottolinea UNDP $(2009,84)$. Le più recenti pubblicazioni su monitoraggio e valutazione nell'ambito dei programmi di reinsediamento affermano ancora che «la diffusione di M\&E è stata finora limitata o lenta nell'ambito dei programmi di reinsediamento in tutto il mondo» (Ahad, Le Coz, Beirens 2020, 4). Ciò è dovuto principalmente a quattro ordini di motivi che «hanno impedito ai Paesi di compiere progressi concreti nella documentazione e nell'analisi delle prestazioni dei loro programmi di reinsediamento» (Ahad, Le Coz, Beirens 2020, 10):

1. la mancanza di una tradizione di M\&E tra gli attori che si occupano dei programmi di reinsediamento, che in parte si spiega con il fatto che le attività di reinsediamento sono abbastanza nuove in molti Paesi dell'UE, compresa l'Italia (dove sono cominciate in modo regolare a partire dal 2015); ${ }^{21}$

2. la valutazione non è vista come una priorità assoluta, dal momento che la preoccupazione principale è stata innanzitutto quella di svolgere le operazioni del programma nel modo più fluido possibile;

3. «lacune e problemi di compatibilità tra i dati disponibili» (Ahad, Le Coz, Beirens 2020, 10): sistemi nazionali di reinsediamento differenti tra loro e dati provenienti da fonti diverse riducono l'interoperabilità dei dati. Ad esempio, non è

19 https://www.oecd.org/dac/peer-reviews/IADB\%202010\%20managing\%20for\%20 results.pdf.

20 http://web.undp.org/evaluation/handbook/documents/english/pme-handbook.pdf.

21 Prima del 2015, l'Italia ha promosso un programma di reinsediamento ad hoc, per circa 180 palestinesi provenienti dal campo di Al Tanf in Siria (nel 2009). Cf. https:// www.unhcr.it/wp-content/uploads/2019/09/Italy-Chapter.pdf. 
sempre operata una chiara distinzione tra i rifugiati reinsediati e i rifugiati che fanno ingresso nel Paese attraverso altri percorsi. ${ }^{22}$ Infine, alcuni mostrano preoccupazioni sul modo in cui i risultati della valutazione potrebbero essere usati o abusati, principalmente in modo politicizzato.

Nonostante queste difficoltà, il monitoraggio e valutazione sono considerati un elemento chiave per sostenere i sistemi di reinsediamento. L'attenzione si concentra sulla valutazione dell'efficacia complessiva di un programma nel raggiungere i suoi obiettivi, e nel garantire la sostenibilità dei precedenti e nuovi programmi di reinsediamento, fornendo evidenze di alta qualità, raccolte con costanza nel tempo (cf. Ahad, Le Coz, Beirens 2020, 1). Dovrebbero essere rese esplicite le ipotesi circa «le correlazioni tra le attività di un programma e le risorse (input), i risultati immediati (output), i risultati a medio termine (outcomes) ed i risultati a più lungo termine (impatto)» (Ahad, Le Coz, Beirens 2020, 12). Inoltre, come suggerito sempre da Ahad, Le Coz e Beirens $(2020,13)$ i sistemi di valutazione M\&E possono essere utilizzati per specifiche parti di un programma e per determinare se quel determinato design possa essere reso più pertinente/appropriato, gli effetti e il rapporto costi-efficacia migliorati e potenziati. In altre parole, $M \& E$ possono contribuire al raggiungimento degli obiettivi strategici e operativi di un programma, migliorando al contempo la comprensione degli ostacoli (sia nel senso di riuscire a definirli sia nel senso di capire come superarli). Pertanto, la ricerca su ciò che funziona, quando, dove e perché, è la chiave per chiarire i molti diversi approcci e modelli di reinsediamento finora implementati nel mondo. Ad esempio, «gli Stati che hanno maggiore esperienza di reinsediamento possono anche utilizzare questo know-how per personalizzare le loro operazioni e il supporto che offrono ai nuovi Paesi di reinsediamento, consentendo loro di scendere in pista schivando errori evitabili» (Ahad, Le Coz, Beirens 2020, 2). Ciò crea le basi per la raccolta e la condivisione di risultati evidence-based, successi e lezioni apprese, aumentando le possibilità di replicare le migliori pratiche. Questo promuove in modo coerente la strategia triennale dell'UNHCR (2019), basata sul Global Compact on Refugees (GCR), che riconosce l'importanza della cooperazione internazionale, di azioni collettive concrete, e di risultati misurabili per giungere a soluzioni sostenibili per le situazioni dei rifugiati. Per quanto riguarda la valutazione, il risultato desiderato è lo sviluppo di una cultura comune di $\mathrm{M} \& \mathrm{E}$. Al fine di raggiungere questo

22 Ad esempio, in Italia «dove il SIPROIMI ha un sistema per monitorare l'integrazione, i suoi dati tipicamente non distinguono tra rifugiati reinsediati e persone che hanno chiesto ed ottenuto l'asilo dopo essere arrivate in Europa» (Ahad, Le Coz, Beirens 2020, 11). 
obiettivo ultimo, la letteratura su M\&E nell'ambito dei programmi di reinsediamento sottolinea la necessità di intraprendere due principali linee d'azione. In primo luogo, l'adozione di framework formali e sistematici di M\&E, essenziali in quanto forniscono «la base di evidenze per analisi sistematiche e miglioramenti» e «rafforzano la memoria istituzionale e facilitano i confronti nel tempo» (Ahad, Le Coz, Beirens 2020, 8). In secondo luogo, le attività di M\&E non dovrebbero limitarsi al monitoraggio degli input e dei risultati immediati, ma dovrebbero poi passare anche alla valutazione d'impatto. Servono infatti ulteriori ricerche sull'impatto del reinsediamento sui rifugiati e sulle comunità ospitanti, al fine di fornire prove documentate sugli aspetti critici e sui benefici del reinsediamento per tutte le parti interessate. Si ritiene che questo sia un aspetto cruciale per garantire un sufficiente sostegno pubblico ai programmi di reinsediamento, tale da renderli politicamente percorribili, e quindi sostenibili, sebbene «sia importante riconoscere che la generazione di dati, in sé e per sé, spesso non è sufficiente a garantire sostegno pubblico e politico al reinsediamento [...] in particolare laddove i programmi di reinsediamento sono politicizzati e polarizzati politicamente» (Ahad, Le Coz, Beirens 2020, 9).

I programmi di reinsediamento si sviluppano attraverso una serie di fasi interconnesse, in successione cronologica, che devono essere monitorate separatamente l'una dall'altra, per poi valutare in seguito i collegamenti tra le varie fasi e verificare le ipotesi su cui si basano i cambiamenti e l'impatto. Quando si sviluppa la metodologia di un framework di M\&E di un programma di reinsediamento è necessario identificare degli indicatori per ciascuna fase del programma, e le proprietà che compongono tali indicatori, al fine di trasformare gli obiettivi in variabili misurabili. Più indicatori vengono sviluppati, più è possibile catturare la complessità della dimensione da analizzare. È importante notare, tuttavia, che non sempre è possibile includere differenti dimensioni all'interno di un sistema di M\&E (cf. Ahad, Le Coz, Beirens 2020, 20). Per questo motivo nella progettazione e nell'implementazione di framework di M\&E, i responsabili della valutazione del programma di reinsediamento dovrebbero prima scegliere quali dimensioni includere, e come farlo, confrontandosi con le esperienze precedenti. Esperienze precedenti simili sono prese come punti di riferimento per disegnare un progetto, allo stesso modo per valutare un progetto normalmente si analizzano pratiche, metodologie e strumenti di valutazione che sono già stati messi in campo nella valutazione di progetti simili.

Nel prosieguo del paragrafo seguente si discuteranno quindi i meriti dell'implementazione di M\&E nei programmi di reinsediamento, concentrandosi su esperienze paragonabili al progetto COMMIT, focalizzando l'attenzione principalmente su due essenziali azioni progettuali: le sessioni di orientamento pre-partenza e il supporto all'in- 
tegrazione post-arrivo. L'OIM considera questi aspetti due fasi chiave di qualsiasi programma di reinsediamento che metta al centro i rifugiati. Non a caso, nelle linee guida per l'implementazione dei programmi di reinsediamento, «l’OIM promuove due approcci di ampio respiro a fondamento di qualsiasi operazione di reinsediamento: programmi centrati sui rifugiati e rafforzamento del legame tra orientamento e supporto pre-partenza e post-arrivo» (IOM 2019a, 34).

Il presente paragrafo si propone di fare luce sui processi di valutazione, specificatamente nell'ambito dei programmi di resettlement, come il progetto COMMIT. Fornendo una cornice teorica sul concetto di valutazione, le sue finalità, la classificazione delle varie tipologie, e infine, i criteri che la regolano. Si farà particolare attenzione alle migliori pratiche emerse da interventi di ricerche valutative. Infine, si propone una disamina sul Pre-Departure Orientation (PDO) e sui programmi di mentorship che ne fanno parte nella fase post-arrivo e lo veicolano, azioni progettuali sviluppate dal gruppo di ricerca coordinato da Antonella Benucci dell'Università per Stranieri di Siena, all'interno del progetto COMMIT.

\section{La valutazione: obiettivi, tipologie e criteri}

La valutazione è stata definita formalmente dal Development Assistance Committee (DAC) dell'Organizzazione per lo Sviluppo Economico (OCSE) nel 1991 e successivamente ripresa nel documento multilingue, Glossary of Key Terms in Evaluation and Results-Based Management, come segue:

The systematic and objective assessment of an on-going or completed project, programme or policy, its design, implementation and results. The aim is to determine the relevance and fulfillment of objectives, development efficiency, effectiveness, impact and sustainability. An evaluation should provide information that is credible and useful, enabling the incorporation of lessons learned into the decision- making process of both recipients and donors. Evaluation also refers to the process of determining the worth or significance of an activity, policy or program. An assessment, as systematic and objective as possible, of a planned, on-going, or completed development intervention. (OCDE 2002, 21)

Trattata anche in un documento dello United Nations Evaluation Group (UNEG), Norms and Standards for Evaluation, a cui organizzazioni come OIM aderiscono, come segue:

An evaluation is an assessment, conducted as systematically and impartially as possible, of an activity, project, programme, strat- 
egy, policy, topic, theme, sector, operational area or institutional performance. It analyses the level of achievement of both expected and unexpected results by examining the results chain, processes, contextual factors and causality using appropriate criteria such as relevance, effectiveness, efficiency, impact and sustainability. An evaluation should provide credible, useful evidence-based information that enables the timely incorporation of its findings, recommendations and lessons into the decision-making processes of organizations and stakeholders. (UNEG 2016, 10)

Le definizioni presentate precedentemente fanno emergere chiaramente qual è lo scopo finale del processo valutativo: fornire informazioni basate sulle evidenze che possono contribuire all'apprendimento organizzativo e arricchire le conoscenze globali per interventi futuri. Come affermato anche da UNEG $(2016,30)$ «la valutazione è un processo decisionale, ma serve piuttosto come input per fornire ai decision makers conoscenze e evidenze di performance e buone pratiche». Andando oltre lo scopo specifico di un determinato intervento di valutazione, l'obiettivo è quello di capire perché e in che misura vengono raggiunti determinati risultati, sia che essi siano attesi che inattesi, ed analizza implicazioni. In altre parole, come ci ricorda l'OIM (IOM 2019b, 172), in un'azione di monitoraggio ci si pongono le domande «Che cosa è stato fatto? Come è stato fatto? Quando è stato fatto?», per ottenere in tempo reale, in una prospettiva a breve termine, informazioni per attuare operazioni correttive, se necessarie, e per fornire un riscontro regolare le azioni di valutazione cercano di dare risposte a queste domande, oltre a riflettere sui quesiti «Perché e quanto bene è stato fatto?». Due azioni che si possono definire quindi interconnesse e complementari e che se non rispettate e non utilizzate parallelamente fin dall'inizio del percorso potrebbero falsare la valutazione finale (cf. ALNAP 2016, 30). Per consentire un'analisi a lungo termine, «la valutazione si basa fortemente sui dati generati attraverso il monitoraggio durante il programma e il ciclo progettuale» (UNDP 2009, 9).

Quindi la valutazione implica un giudizio sul valore dell'attività e dei suoi risultati, ma al contempo «non dovrebbe essere considerata puramente come un momento giudicante, ma come un'opportunità di crescita e di miglioramento» (Saggiomo 2019, 8). Come sottolineato da ALNAP $(2016,18)$ le azioni valutative vengono spesso percepite come un momento di critica e temute da chi vi si sottopone, mentre invece dovrebbero essere vissute come una possibilità di crescita costruttiva e quindi utile. Questo aspetto di giudizio che spesso tende ad essere frainteso e vissuto negativamente, per quanto sia basato su criteri scientifici di ricerca, va inteso come un'opportunità di riflessione interna rivolta esclusivamente ad uno specifico intervento che deve essere, sta o è stato attuato, e quindi come una fonte utile 
ad estrapolare informazioni in prospettiva futura per diventare una linea guida per future azioni di sviluppo. Pertanto, la valutazione ha lo scopo di stabilire future raccomandazioni e tenere traccia delle migliori pratiche sulla base delle quali poter poi fondare future iniziative e programmazioni, progettate, implementate e migliorate, siano queste nello stesso contesto o in altri contesti.

I risultati della valutazione dovrebbero alimentare il futuro lavoro umanitario, informando il processo decisionale, facilitando l'apprendimento, identificando le aree di miglioramento e, in particolare, indicando altre possibili domande di ricerca o di valutazione. (UNEG 2016, 30)

In quanto tali, sono state descritte dall'OIM come «strumenti di cambiamento» (IOM 2011, 396), che evitano una prospettiva centrata esclusivamente sul singolo intervento o istituzione, prediligendo la creazione e la condivisione di un valore aggiunto.

Per chiarire meglio i principali aspetti del concetto di valutazione bisogna innanzi tutto comprendere le diverse tipologie di interventi che può comprendere, se è umanitario o di sviluppo, ad esempio un progetto, un programma, una politica, una strategia, un'area tematica, un'assistenza tecnica, una consulenza politica, un'istituzione, un meccanismo di finanziamento, uno strumento ecc.

Si può definire come un processo sistematico che ha l'esigenza di essere pianificato dettagliatamente nelle prime fasi progettuali e che ha come focus quello di fungere da guida per future iniziative. Il momento in cui viene condotta la valutazione può variare in base agli obiettivi specifici di diversi interventi perché i tempi di una valutazione dovrebbero essere direttamente legati al suo scopo e al suo utilizzo. Sulla base di questi fattori, l'azione valutativa può essere condotta in diverse fasi del ciclo intero di un progetto. In primo luogo:

- ex-ante evaluation, che corrisponde solitamente ad uno studio sulla fattibilità, con l'obiettivo di valutare se una proposta progettuale può avere successo prima del suo inizio effettivo, determinando la sua rilevanza e pertinenza;

- mid-term evaluation, che ha luogo durante l'implementazione del progetto e solitamente si espande per un periodo significativamente lungo, o in un progetto pilota, innovativo che ancora non è stato sperimentato. Consente di apportare correzioni in corso d'opera se necessario, risultando assimilabile al monitoraggio, completando la valutazione e focalizzandosi sul breve e medio termine per poter incidere apportando cambiamenti all'interno del programma, quindi «in una valutazione di medio termine l'attenzione si sposta sull'efficacia, sui risultati e sulle raccomandazioni per il miglioramento» (IOM 2011, 378); 
- final evaluation, la tipologia più frequente, condotta una volta concluso il programma per verificare fino a che punto gli obiettivi di sviluppo sono stati raggiunti e riflettere sulle esperienze positive e negative incontrate durante l'implementazione del progetto, per le pianificazioni future. Essendo la tipologia che meglio riflette l'obiettivo ultimo di un programma, come sottolineato dall'OIM «le valutazioni finali sono in grado di verificare meglio la sostenibilità e l'impatto» (2011, 378);

- ex-post valuation, condotta un po' di tempo dopo la conclusione di un progetto, utile a valutare l'impatto a lungo termine.

In secondo luogo, quelli che sono i criteri di valutazione che riportiamo di seguito, standardizzati e proposti dall'OCSE/DAS per fornire una base per interventi valutativi, ma come evidenziato dall'OIM «non tutte le valutazioni devono concentrarsi su tutti questi criteri. A seconda dello scopo della valutazione, potrebbero esserne valutati solo alcuni» (IOM 2019b, 187):

- la rilevanza, si riferisce alla misura in cui gli obiettivi di un programma e il modo in cui sono stati implementati corrispondono alle esigenze dei beneficiari e degli stakeholder, pertanto viene definita come «la misura in cui gli obiettivi e la progettazione di un intervento rispondono ai bisogni, alle politiche e alle priorità dei beneficiari, globali, nazionali e dei partner/delle istituzioni, e continuano a farlo se le circostanze mutano» (OECD 2019a, 7);

- l'efficacia è rivolta all'effettivo raggiungimento degli obiettivi e dei risultati previsti in un intervento tenendo sempre presente la loro importanza e includendo potenziali risultati distinti fra i gruppi. ${ }^{23}$ Pertanto, si riferisce all'analisi dei progressi che servono a, come suggerito dall'OIM (IOM 2011, 373), determinare se le attività svolte hanno prodotto output e se il raggiungimento di questi ha portato a gli outcome che erano stati previsti;

- l'efficienza riguarda le risorse utilizzate volte al raggiungimento dei risultati attesi, in particolare risorse economiche ed input (fondi, competenze, tempo ecc.), e la misura in cui tali risorse vengono fornite attraverso gli interventi. ${ }^{24}$

- l'impatto utile a valutare effetti sociali, ambientali o economici prodotti, in grande scala «effetti a lungo termine, positivi e negativi, primari e secondari, prodotti da un intervento di sviluppo, direttamente o indirettamente, intenzionali o non intenzionali» (OECD 2019a, 11). Utile ad esaminare gli effetti e i 
cambiamenti maggiori a cui un intervento può servire, ${ }^{25}$ contrariamente all'efficacia.

- la sostenibilità come descritto da Spitz et al. (2013) misura se, su varie dimensioni (finanziaria, economica, sociale e ambientale), e fino a che punto gli effetti di un intervento dureranno nel tempo, nello specifico la continuità dei benefici di un determinato intervento in conseguenza al completamento dell'assistenza. ${ }^{26}$

- la coerenza un criterio che riguarda l'importanza della complementarità, dell'armonizzazione e del coordinamento interno, oltre a valutare «la compatibilità dell'intervento con altri interventi in un Paese, settore o istituzione» (OECD 2019a, 8). Tutti aspetti fortemente promossi dalla cultura della condivisione di risultati basati sulle evidenze, nel caso della coerenza esterna, mentre la coerenza interna si riferisce agli interventi condotti dalla stessa istituzione/governo, alla luce delle norme e degli standard internazionali a cui aderisce.

Per concludere, in terzo luogo, la metodologia di valutazione richiede imparzialità e oggettività per non perdere credibilità ed evitare la distorsione dei risultati, dell'analisi e delle conclusioni. Aspetto quest'ultimo importante perché un intervento di valutazione non dovrebbe fornire unicamente informazioni in linea con gli scopi dell'intervento stesso, ma stabilire anche una guida per future procedure.

\section{La valutazione del Pre-Departure Orientation}

Sulla base della letteratura disponibile e delle evidenze emerse da precedenti programmi di reinsediamento che implementano attività di PDO, si cercherà ora di chiarire quali strumenti, criteri e metodi sono stati maggiormente utilizzati nella valutazione di tali percorsi, e quali sono le raccomandazioni e le conclusioni più rilevanti messe in risalto a seguito delle valutazioni.

È possibile evidenziare alcune tendenze comuni nel processo di valutazione dei programmi di PDO. Innanzitutto, la valutazione viene condotta dopo l'implementazione delle attività, quindi corrisponde ad una valutazione finale, che ha come focus quello di valutare l'efficacia e l'impatto del PDO. In altre parole, si tratta di guadagnare «una posizione informata e consapevole riguardo a sé i corsi di formazione stanno raggiungendo gli obiettivi prefissati ed in che modo i rifugiati reinsediati si integrano nel tempo» (Fratzke, Kainz 2019, 30), comprendendo in particolare fino a che punto il PDO svolge un ruolo 


\section{6 - Tecniche e strumenti per la gestione della relazione mentor-rifugiato}

nel processo di integrazione. Tali scoperte hanno lo scopo di guidare la progettazione di futuri percorsi di orientamento, in una prospettiva lungimirante. Considerando le tempistiche della valutazione, mentre l'efficacia può essere valutata immediatamente dopo la fine delle sessioni (attraverso feedback finali), l'impatto è un aspetto di lungo termine. Per questo motivo parti della valutazione vengono condotte in diversi momenti nel tempo, anche dopo che i rifugiati arrivano nel Paese di reinsediamento. Ad esempio, IOM Romania distribuisce questionari che vengono richiesti ai rifugiati di compilare per la prima volta immediatamente dopo aver partecipato all'orientamento e poi di nuovo 45 giorni dopo il loro arrivo in Romania (Fratzke, Kainz 2019, 31). Le interviste di follow up con i rifugiati e altri stakeholder, rilevate qualche tempo dopo il loro arrivo nel Paese di reinsediamento, sono considerate uno strumento efficace per ottenere una visione più accurata di quanto sia utile il programma pre-partenza.

Per quanto concerne la metodologia di ricerca, alcuni Stati di reinsediamento raccolgono feedback informalmente, basandosi sull'osservazione dei formatori o sui commenti dei rifugiati durante o dopo la formazione. Ad esempio, alla fine del programma possono svolgersi discussioni tra formatori e partecipanti sui risultati della formazione. ${ }^{27}$ Se osservazioni dirette sull'erogazione del servizio, sia di persona che da remoto, ${ }^{28}$ sono tra gli strumenti di ricerca utilizzati per valutare il PDO,

l'uso di soli metodi informali per monitorare un orientamento [...] crea il rischio che qualsiasi lezione appresa, potenziale problema, o successi non saranno o saranno solo parzialmente ricondotti alla più ampia progettazione ed implementazione del programma. (Fratzke, Kainz 2019, 31)

Per di più, tale prassi non solo ridurrebbe i benefici della valutazione per il Paese o l'organizzazione che attuano il programma di orientamento, ma limiterebbe anche lo sforzo di condividere pratiche promettenti al di là dell'ambito di un intervento specifico, su cui si potrebbero basare linee guida più generali. Dall'altro lato, le procedure formali possono richiedere sia tempo che denaro, in particolare in

27 È il caso del Norwegian Cultural Orientation Programme (NORCO). Cf. https:// tinyurl.com/2s3r3383.

28 I programmi di orientamento possono aver luogo in molteplici forme, a causa di vari fattori: la sicurezza sia del fornitore di CO sia dei rifugiati e le difficoltà logistiche (non sempre possono essere assicurati programmi in presenza). Le soluzioni alternative sono le formazioni a distanza con sessioni online o telefoniche. Ad esempio, negli Stati Uniti il Cultural Orientation Resource Exchange (CORE) ha sviluppato una guida e un programma per condurre un $\mathrm{CO}$ telefonico per i rifugiati da reinsediare. In Canada, l'IRCC Evaluation Team ha suggerito di combinare modelli di erogazione web-based e in presenza, seppur assicurando un equilibrio tra i due (IRCC 2018). 
termini di risorse umane. Quando la valutazione viene effettuata con mezzi formali gli strumenti di ricerca più spesso adottati sono questionari di sondaggio del feedback, interviste e focus group. I questionari possono essere amministrati di persona o online, o in entrambe le modalità, a seconda del momento della somministrazione. Poiché si tratta di un processo di valutazione in più fasi, agli stessi stakeholder possono essere sottoposti nel tempo più strumenti di ricerca per valutare diversi fattori e raccogliere dati sia qualitativi che quantitativi. I sondaggi vengono solitamente somministrati in più lingue o con il supporto di interpreti. Anche le interviste, i focus group e le discussioni sono strutturati utilizzando una serie standard di domande, predisposte su misura per adattarsi ai vari ruoli degli attori coinvolti nel processo di valutazione.

Indipendentemente dalla metodologia utilizzata per valutare il PDO, i dati sono idealmente raccolti da una vasta gamma di prospettive, per avere molteplici linee di evidenza. Ciò significa che nel processo di valutazione è coinvolta un’ampia gamma di attori, compresi, normalmente, i beneficiari del reinsediamento che hanno partecipato al programma (individualmente o in piccoli gruppi, come famiglie), i formatori e il personale delle strutture di accoglienza, così come altri principali stakeholder nel Paese di reinsediamento quali autorità locali, organizzazioni della società civile, volontari e responsabili politici. Per valutare meglio l'impatto, può essere previsto anche un gruppo di confronto di rifugiati che non hanno avuto accesso al PDO. ${ }^{29}$

L'efficacia dei corsi di PDO è valutata principalmente in termini di chiarezza, esaustività, pertinenza e utilità delle informazioni fornite durante il PDO. Ai partecipanti viene solitamente chiesto di esprimere il proprio livello di soddisfazione riguardo al contenuto della formazione, ad esempio classificando ciascuno degli argomenti discussi come di grande, media o poca importanza. ${ }^{30}$ Viene inoltre chiesto loro di identificare eventuali argomenti nuovi o aggiuntivi da trattare, al fine di evidenziare potenziali lacune informative su aspetti di loro interesse, o se la discussione su specifici argomenti esistenti debba essere ulteriormente estesa. Ad esempio, diversi report di valutazione di CO dimostrano che i partecipanti richiedono spesso maggiori dettagli su alloggi, sostegno finanziario e opportunità di lavoro, ${ }^{31}$ se il curriculum è in linea con le esigenze dei rifugiati o se sono neces-

29 Ad esempio, nella Valutazione dei Servizi di Insediamento Pre-Arrivo condotta dall'IRCC Evaluation Team in Canada ad aprile 2018, la metodologia prevedeva un confronto tra beneficiari e non beneficiari. Cf. IRCC 2018.

30 Ad esempio, nel caso della valutazione dell'Australian Cultural Orientation (AUSCO) program (2009), sia i beneficiari sia i formatori dell'OIM esprimono il loro feedback sull'utilità del contenuto classificando ogni argomento come di grande, media o poca importanza. Cf. AUSCO, s.d.

31 Cf. IOM 2020b; IRCC 2018; AUSCO, s.d. 
sarie alcune modifiche al programma. ${ }^{32}$ Nel processo di valutazione, è essenziale valutare fino a che punto il PDO è adattato alle esigenze di specifici gruppi di rifugiati considerati più vulnerabili, nei confronti dei quali è quindi richiesta un'attenzione speciale. Questo è in particolare il caso di donne e di giovani, ma altri sottogruppi possono essere ugualmente identificati, per esempio facendo una distinzione tra adolescenti/giovani adulti e bambini, e tenendo in considerazione le esigenze specifiche di persone analfabetizzate o delle famiglie in quanto differenti. ${ }^{33}$ Gruppi di rifugiati con caratteristiche sociodemografiche diverse possono avere percezioni diverse dell'efficacia del PDO in termini di rilevanza delle informazioni fornite.

Oltre al feedback sul contenuto del PDO, vengono raccolte anche le percezioni dei partecipanti circa le modalità di erogazione, per accertarsi che gli stili di insegnamento siano efficaci e contribuiscano alla creazione di un ambiente di apprendimento non minaccioso. I formatori sono spesso interrogati anch'essi circa la loro percezione del contenuto del curriculum e delle modalità di erogazione. Le linee guida internazionali raccomandano che la natura dei corsi segua un approccio centrato sullo studente e partecipativo. In altre parole, i corsi del PDO dovrebbero essere esperienziali, favorendo il coinvolgimento attivo dei partecipanti e promuovendo il lavoro di gruppo tra pari.

Una metodologia didattica interattiva che includa discussioni e giochi di ruolo è necessaria per aumentare l'effettiva acquisizione dei contenuti. L'uso di input iconografici e di strumenti multimediali come i video è fortemente consigliato. Mostre e video di storie di reinsediamento di successo dove i protagonisti sono rifugiati reinsediati che attualmente abitano nel Paese di reinsediamento è una pratica promettente, tradizionalmente promossa da Paesi di reinsediamento di lunga data come gli Stati Uniti ed attualmente implementata da diverse nazioni nel mondo all'interno delle attività di PDO. A questo proposito, è interessante notare come formatori e partecipanti possano avere opinioni diverse circa specifici stili di insegnamento. Per esempio, giochi di ruolo, drammatizzazioni e simulazioni non sono particolarmente popolari tra i partecipanti per ragioni culturali.

Per garantire l'efficacia dei corsi e favorire la comprensione del contenuto tenendo allo stesso tempo presente il modo di pensare e imparare culturalmente determinato dei rifugiati, l'OIM promuove il coinvolgimento di formatori bi-culturali. Nel contesto europeo, il primo programma di PDO ad adottare l'uso esclusivo di formatori biculturali è stato il Cultural Orientation for Norway Bound Refugees

32 Per allineare i curricula di PDO ai bisogni dei rifugiati, prima del PDO può essere condotta un'analisi dei bisogni per mappare il profilo dei rifugiati.

33 Il programma di orientamento culturale australiano (AUSCO), per esempio, ha introdotto delle sessioni specifiche per il 'Family Day' all'interno dell'orientamento culturale. Cf. AUSCO, s.d. 
(NORCO). Idealmente, i formatori dovrebbero essere persone con lo stesso background culturale e linguistico dei rifugiati da reinsediare: il coinvolgimento di rifugiati reinsediati da tempo come formatori aiuta a migliorare la credibilità delle informazioni e l'accettazione dei contenuti e delle metodologie di erogazione degli stessi. Inoltre, formatori bi-culturali possono tenere i corsi del PDO nella lingua nativa dei rifugiati, come suggerito sia dall'OIM sia da ex partecipanti di sessioni di PDO nel corso di precedenti valutazioni. ${ }^{34}$

Per quanto riguarda la valutazione d'impatto, l'efficacia nel gestire le aspettative dei rifugiati è uno dei principali aspetti da valutare, andando ad osservare la corrispondenza tra le aspettative dei rifugiati e le loro percezioni delle condizioni di vita nel Paese di reinsediamento post-arrivo. Il riscontro fornito dai partecipanti è sempre usato in una prospettiva lungimirante, per migliorare il PDO. Una pratica particolare è stata introdotta nell'ambito di AUSCO, prevedendo il ritorno di rifugiati reinsediati nei loro Paesi di partenza per incontrare i formatori che erogano l'orientamento culturale e condividere i loro pensieri su argomenti e informazioni che richiedono maggiore attenzione. ${ }^{35}$ Un ulteriore beneficio della valutazione d'impatto del PDO è quello di fornire raccomandazioni per un maggior coordinamento e miglioramento sia dei programmi di orientamento pre-partenza, sia di quelli post-arrivo, garantendo il loro allineamento ed equilibrio.

Il gruppo di ricerca dell’Università per Stranieri di Siena, coordinato da Antonella Benucci e impegnato nel progetto COMMIT, si è impegnato nella stesura di una metodologia di valutazione nell'ambito delle sessioni PDO, con l'aiuto di un campione di formatori che si occupano di questi programmi, nei quattro Paesi target oggetto di reinsediamento: Croazia, Italia, Portogallo e Spagna. L'obiettivo primario che il team si è prefissato è stato quello di garantire la solidità dell'approccio metodologico proposto per l'analisi dei risultati della valutazione, sulla base di strumenti sia quantitativi che qualitativi, con il supporto di sessioni di monitoraggio e lo studio di pratiche di valutazione della PDO già esistenti in Italia e in Portogallo. Un processo pensato oltre che per garantire dei feedback post-arrivo da parte dei rifugiati sulle sfide che si sono trovati ad affrontate in assenza di programmi di orientamento, anche per approfondire e contribuire all'arricchimento della richiesta di informazione prima della partenza.

La finalità del lavoro dell'Università per Stranieri di Siena, con il supporto di OIM, è stata quella di implementare una serie di strumenti di valutazione e autovalutazione che, sulla base di una solida metodologia scientifica, e grazie alla pregressa esperienza nel set- 
tore della migrazione, consentono la valutazione di tutti i fattori che comprendono e che possono influenzare una sessione PDO.

La sostanziale differenza fra questa proposta di intervento valutativo e altri progetti di ricerca, è intrinseca all'elaborazione degli strumenti, che sono stati pensati per raccogliere informazioni su una realtà che risulta essere ancora in via di sviluppo e per confrontare l'evoluzione avvenuta in due momenti differenti delle attività volte al reinsediamento: le attività di Pre-Departure Orientation e quelle di Post-Arrival Orientation. Oltre all'implementazione di strumenti sia di tipo quantitativo che qualitativo, sono stati inoltre definiti anche gli obiettivi e i parametri di valutazione sulla base delle aspettative che il programma PDO intende raggiungere (cf. Monaci 2021).

Gli strumenti di valutazione sopra citati sono di 7 tipologie, rivolti ai principali attori coinvolti nei programmi di reinsediamento:

- strumenti dedicati ai rifugiati: il Questionario per beneficiari del resettlement (ex ante ed ex post) e l'Intervista per beneficiari del resettlement (ex post) pensati per misurare le aspettative dei beneficiari nei confronti dei contenuti e dell'organizzazione delle attività PDO, e quanto possa aiutare i rifugiati nel processo di reinsediamento;

- strumenti dedicati ai formatori PDO: il Questionario per formatori PDO (ex ante ed ex post), l'Intervista per formatori PDO (ex post), la Griglia di autovalutazione per formatori PDO (ex ante, mid-term ed ex post) e la Griglia di analisi dei materiali didattici per attività PDO, concepiti per valutare il rispetto delle aspettative in merito ai contenuti e all'organizzazione delle attività PDO, oltre a verificare se le modalità e l'approccio della loro partecipazione all'orientamento culturale possono essere utili ai beneficiari;

- strumento per operatori (staff) PDO: il Questionario per operatori PDO (ex post) ha lo scopo di condurre un sondaggio sulle aspettative dell'orientamento culturale che gli operatori responsabili della delocalizzazione si sono preposti per quanto concerne le attività, i contenuti e i metodi ritenuti più funzionali e idonei;

- strumenti per comunità ospitanti/mentors: il Questionario per comunità ospitante/mentors (ex ante ed ex post) e l'Intervista per comunità ospitante/mentors (ex post) sono volti a comprendere le aspettative dei migranti in merito all'organizzazione dei programmi di reinsediamento e i contenuti che li riguardano. La valutazione di questo strumento è indirizzata verso il grado di interesse dei cittadini a partecipare sia alla pianificazione che alla realizzazione della PDO, oltre allo sviluppo personale di conoscenze sul tema della migrazione e reinsediamento. 
Monitoraggio e valutazione dei programmi di mentorship post-arrivo

A causa della scarsa presenza di ricerche sul mentoring e per il fattore innovativo di tale azione, è difficile effettuare una panoramica sulle pratiche di valutazione dei programmi di mentorship.

In alcuni Paesi come il Canada da decenni i cittadini si impegnano nell'accoglienza dei rifugiati, trovando una loro propria espressione in private or community-based sponsorships. In altri, invece, il coinvolgimento dei membri della società civile rimane informale, anziché divenire una sistematica offerta di integrazione per i gruppi di rifugiati reinsediati. Questo a conferma del fatto che i programmi di mentorship non sono sempre presenti all'interno degli schemi di reinsediamento statali.

Pertanto, si può affermare che un buon numero di programmi attualmente in corso è rappresentato da progetti pilota che mirano a documentare la metodologia e gli aspetti intrinsechi della progettazione, e a testimoniare la necessità di approfondire l'implementazione di tali programmi a dimostrazione dell'efficacia di questo strumento.

Entrando nello specifico della valutazione dei programmi di mentorship, in evoluzione, poche sono le informazioni disponibili circa i processi valutativi condotti fino ad ora. Si può però in questa sede evidenziare che la rete SHARE, facente parte dello European Resettlement Network (ERN), nel 2013, «ha identificato il volontariato come un'area prioritaria per lo sviluppo nell'ambito del reinsediamento e dell'integrazione di rifugiati» (ICMC 2015, 2).

Per poter valutare a pieno l'efficacia e la qualità di un programma di mentorship devono essere considerati anche alcuni aspetti critici, come suggerito da precedenti progetti pilota. In primo luogo, se l'abbinamento mentors-mentees si rivela confacente per entrambi, e se il livello di fiducia reciproca è equilibrato. In secondo luogo, la possibile presenza di barriere linguistiche fra i due attori o di incomprensioni interculturali, e come esse vengono gestite. In terzo e ultimo luogo, informazioni riguardo all'organizzazione e il coordinamento delle attività, per valutare l'appropriatezza del supporto da essi ricevuto.

Analizzando più da vicino la metodologia di ricerca e valutazione, i questionari di soddisfazione possono anche essere somministrati per iscritto, solitamente online, ma difficilmente rappresentano l'unico strumento di ricerca/valutazione e la somministrazione avviene sia ai mentors che ai mentees individualmente. La valutazione congiunta, rivolta ad entrambe le parti, può rappresentare un momento di riflessione simultanea sul processo di mentorship, in particolare in termini di raggiungimento degli obiettivi prefissati, come nel caso del Mentoring Program for Migrants (Portogallo, 2014). ${ }^{36}$

36 https://ec.europa.eu/migrant-integration/intpract/mentoring-programfor-migrants. 
Entrando più nel dettaglio, i dati quantitativi raccolti con i questionari si riferiscono principalmente ad alcuni criteri generici:

- numero dei partecipanti coinvolti di entrambe le parti;

- profilo dei mentors e dei mentees implicati nelle attività,

- frequenza e durata delle attività e degli incontri;

- numero di relazioni di mentoring interrotte e di quelle portate a termine.

Gli strumenti maggiormente impiegati sono quelli di tipo qualitativo, le interviste e la raccolta orale di feedback, per eludere le difficoltà che potrebbero incontrare alcuni rifugiati nella compilazione dei questionari scritti. Poiché alcuni programmi non richiedono la presenza costante di mentees, mentors e staff composti da professionisti contemporaneamente, la maggior parte delle interviste viene condotta da remoto, attraverso il telefono o via e-mail. Purtroppo, però, la letteratura di riferimento sui programmi di mentorship è carente di dettagliati riferimenti sui protocolli delle interviste, si sa però che questo tipo di valutazione risulta essere prevalentemente di tipo qualitativo in quanto i risultati vengono tendenzialmente riportati in maniera più descrittiva che numerica. Tale tipologia di valutazione mira a: valutare la percezione del valore e dell'impatto umano e sociale, sia dei mentors che dei mentees; documentare la progettazione e l'implementazione del programma per la trasferibilità; supportare richieste e finanziamenti per iniziative future; costruire sostegno pubblico al volontariato attraverso advocacy e campagne di comunicazione basate sui risultati evidence-based raccolti.

La valutazione dell'efficacia e della qualità mira, dunque, a comprendere in che modo i mentees beneficiano del programma e si integrano meglio grazie al rapporto di mentoring. Inoltre, una valutazione iniziale delle competenze e delle caratteristiche dei mentors, così come delle esigenze dei rifugiati, dovrebbe essere condotta prima dell'inizio del percorso in modo da poter selezionare con criterio dei volontari e aumentare le possibilità di un buon abbinamento fra i partecipanti.

Oltre allo strumento della valutazione, pur risultando complementare, si sente l'esigenza di utilizzare anche quello del monitoraggio, utile a calcolare i progressi del lavoro dei mentors e dei mentees, il loro apprendimento continuo, la crescente evoluzione dei loro bisogni e del loro coinvolgimento in un processo di riflessione costante e reciproco, e a garantire azioni correttive per il proseguimento degli obiettivi.

Essendo la modalità di monitoraggio utile a verificare la relazione di mentorship con focus sullo sviluppo di un forte legame fra le due parti, è importante porre l'attenzione sulla valutazione formativa dei due attori coinvolti nel rapporto di mentoring, attraverso anche l'uso di strumenti di autovalutazione durante la formazione ini- 
ziale e ad intervalli prestabiliti lungo la durata del programma, per verificare l'andamento sull'acquisizione delle competenze e i potenziali bisogni di apprendimento che possono scaturire durante lo svolgimento dello scambio formativo.

Un ulteriore strumento che può rappresentare un sussidio è la stesura di un report periodico sui progressi di entrambe le parti, benché possa rivelarsi un carico di lavoro aggiuntivo per i mentors che ricordiamo essere per la maggior parte volontari. In precedenti progetti di mentorship, è stato chiesto sia a mentors che a mentees di fornire una relazione scritta con delle attività condotte in ogni incontro e un riscontro sulle stesse. Per esempio, nell'ambito del progetto Learning to Be a Good Mentor (LeGMe), il cosiddetto metodo 'stella' è stato utilizzato come strumento di autovalutazione per i mentori, per valutare il miglioramento delle proprie competenze per tre volte: all'inizio, a metà e alla fine del programma. Una scheda con la raffigurazione di una stella è stata consegnata ad ogni mentore. Ogni punta della stella corrispondeva ad una delle nove competenze il cui miglioramento era ritenuto necessario per la buona riuscita del progetto. I mentori potevano aggiungere un'ulteriore competenza. In tale progetto, altri strumenti di valutazione efficace erano il Quaderno del mentor e il Quaderno del mentee: sia ai mentors sia ai mentees veniva chiesto di scrivere una pagina per incontro per descrivere le attività e fornire il proprio riscontro.

Un ulteriore strumento è far riportare dai mentors delle istantanee delle loro esperienze, che fungono da casi studio, come ad esempio accade nel Regno Unito (cf. ICMC, Vluchtelingen Werk Limburg 2014).

In seno al quadro generale dei risultati raggiunti e della sperimentazione effettuata con il progetto COMMIT si illustrerà in questo capitolo il questionario di autovalutazione «Chi è un buon mentore», proposto in occasione della giornata di formazione dedicata ai mentori realizzata nell'ambito del già citato progetto, con lo scopo di autovalutare il proprio livello di sensibilità e di preparazione interculturale.

A nostro avviso è adatto a tutti coloro che a vario titolo entrano o entreranno in contatto con le problematiche connesse alla migrazione, siano essi operatori, formatori, insegnanti, docenti universitari, studenti dei corsi di laurea, studiosi, ricercatori e volontari come i mentori. Questo strumento è stato pensato per essere utilizzato prima dell'inizio della formazione, ma può essere ulteriormente utilizzato in momenti differenti per l'automonitoraggio nel corso delle proprie esperienze. È composto da 26 items, suddivisi in quattro parti o sezioni. La prima riguarda i dati personali, la seconda le funzioni del mentore e del mentoring, la terza la formazione per i mentori e la quarta, infine, le relazioni dei mentori con i mentees. Contiene items utili a delineare un quadro biografico (sesso, età, provenienza, ambiente familiare, lavoro svolto nel Paese di origine, periodo di sog- 
giorno in Italia); di tipo sociolinguistico, riguardo alla lingua materna, alla conoscenza di altre lingue ecc.

Il seminario, pur avendo come finalità quella di illustrare gli obiettivi e le azioni del progetto COMMIT, ha tuttavia consentito di ampliare le tematiche strettamente connesse al progetto, nella direzione della disseminazione non soltanto di risultati ma anche di ipotesi di futuri percorsi, metodologie e strumenti di lavoro. Da un iniziale focus sui bisogni di mentor e rifugiati, i contenuti degli interventi e le discussioni che ne sono scaturite hanno permesso di delineare un quadro generale di offerta formativa rivolta in senso lato a tutti i soggetti che si occupano di tematiche migratorie (operatori, formatori, insegnanti di Italiano LS e L2, studenti di corsi di laurea, ricercatori, studiosi).

Si ritiene che con questi seminari il progetto COMMIT si sia arricchito di riflessioni per future azioni e attività che possano rispondere ai cambiamenti strutturali dei pubblici migranti, dei loro bisogni e necessità e che possano rappresentare uno stimolo per il miglioramento delle politiche di istituzioni e organismi che lavorano nel campo dell'intervento umanitario.

\subsection{Analisi dati questionario di autovalutazione}

Il presente paragrafo descrive i risultati dell'indagine campionaria condotta nell'ambito del progetto COMMIT. La base della rilevazione è un questionario statistico di autovalutazione «Chi è un buon mentore?» somministrato ai partecipanti del ciclo di seminari Mentorship, mediazione e inclusione.

Ogni sezione contiene un set di domande con tipologia varia di risposta, ma per la maggioranza trattasi di risposte chiuse a scelta multipla o con scale di gradimento, ogni sezione tratta di specificità diverse riferendosi a tipologie di dati sia qualitativi che quantitativi.

L'analisi e l'elaborazione dei dati ai fini di elaborazione statistica ha seguito le seguenti fasi:

- acquisizione di tutti i questionari disponibili (compilati);

- valutazione generalizzata della qualità delle risposte fornite nel questionario;

- raccolta su tabella Excel dei dati dei questionari suddivisi in strutture logiche;

- elaborazione queries di selezione secondo i principali incroci selezionati;

- elaborazione sempre in Excel dei dati tabellari per fornire un quadro di sintesi dei dati raccolti di maggiore interesse.

Dopo una prima valutazione generale, si può affermare che:

- non tutti i partecipanti del corso hanno aderito alla compilazione del questionario; 
- nella sezione A del questionario risultano delle domande costruite non efficacemente per l'analisi statistica dei dati, in quanto non avendo previsto una selezione di risposte a domanda chiusa, si è rilevato una sostanziale non omogeneità che ha reso difficile il lavoro di analisi: D1, D4, D5, D6, D8 e D9;

- alcune domande sono state escluse dalla presente analisi in quanto non risultava esserci variabilità nelle risposte fornite.

Fra le varie figure professionali che hanno preso parte alla compilazione del questionario, si è individuato quelle più attinenti al contesto di ricerca: operatori sociali, mediatori linguistico-culturali (assistenti alla comunicazione, educatori interculturali, educatori istituzionali) e insegnanti di italiano L2/LS.

Tabella 1 Risultati domanda 9 (PARTE A Questionario)

\begin{tabular}{lcc}
\hline Occupazione attuale (D9) & Numerosità & Percentuale \\
\hline Insegnante di italiano L2/LS & 5 & $38,4 \%$ \\
\hline Mediatore linguistico-culturale & 4 & $30,8 \%$ \\
\hline Operatore sociale & 4 & $30,8 \%$ \\
\hline
\end{tabular}

Il primo gruppo di domande (D1-14, PARTE A) è servito a fornire indicazioni generali sull'identità socioanagrafica e professionale degli utenti, come per esempio nazionalità, sesso, età, titolo di studio ecc. Dall'analisi dei dati [tab. 2] è possibile affermare che sul totale dei partecipanti il 69,2\% sono di nazionalità italiana, mentre la restante percentuale $(30,8 \%)$ di nazionalità straniera, fra le varietà che si è potuto distinguere: tunisina, portoghese ed italo-camerunense (D1). La fascia di età predominante $(46,1 \%)$ è quella compresa fra i 36 e i 45 anni (D.2), di genere femminile (69,2\%; D3).

Per ciò che concerne la formazione professionale (D4; D5), il 38,4\% dichiarano di essere in possesso del Diploma di Laurea Magistrale/ vecchio ordinamento e in altra percentuale consistente, 30,8\%, di aver conseguito un Master di I/II livello, tasso equivalente nella seconda domanda dove asseriscono di non possedere altri titoli di studio o professionali.

Per poter avere una mappatura dettagliata sul piano linguistico e relative competenze (D6; D7; D8), è stata indicata come Lingua Madre (LM/L1), non a sorpresa visto l'ingente numero di dichiaranti con nazionalità italiana, l'italiano dal 69,2\% degli utenti, e la conoscenza di due lingue oltre alla propria L1, che da un incrocio sono risultate essere l'inglese, $35,7 \%$, e il francese, $28,6 \%$.

Dato interessante rilevato è il bagaglio professionale: solo il $61,5 \%$ dei rispondenti ha vissuto all'estero per un tempo prolungato, ma 
quasi il totale $(84,6 \%)$ ha avuto una discussione con una persona di lingua e cultura diversa dalla loro. Altresì una minima percentuale, $38,4 \%$ ha avuto precedenti esperienze in programmi formali di mentorship e con un tasso leggermente più elevato, 53,8\%, i partecipanti hanno avuto precedenti esperienze in attività informali di mentoring. Aspetto di interesse per il contesto di indagine è che solo il 23,1\% dei soggetti presi in esame è stato in precedenza mentees di qualcuno che a loro volta ha fatto da mentors.

Tabella 2 Risultati sezione 'Dati personali' (parte A del Questionario)

\begin{tabular}{lcc}
\hline Nazionalità (D1) & Numerosità & Percentuale \\
\hline Italiana & 9 & $69,2 \%$ \\
\hline Straniera & 4 & $30,8 \%$ \\
\hline di cui: tunisina & $(2)$ & $(15,4 \%)$ \\
\hline \multicolumn{1}{c}{ portoghese } & $(1)$ & $(7,7 \%)$ \\
\hline italo-camerunense & $(1)$ & $(7,7 \%)$ \\
\hline
\end{tabular}

\begin{tabular}{lcc}
\hline Età (D2) & Numerosità & Percentuale \\
\hline $8-25$ & 1 & $7,7 \%$ \\
\hline $26-35$ & 2 & $15,4 \%$ \\
\hline $36-45$ & 6 & $46,1 \%$ \\
\hline $46-55$ & 2 & $15,4 \%$ \\
\hline+55 & 2 & $15,4 \%$ \\
\hline
\end{tabular}

\begin{tabular}{lcc}
\hline Sesso (D3) & Numerosità & Percentuale \\
\hline$M$ & 4 & $30,8 \%$ \\
\hline F & 9 & $69,2 \%$ \\
\hline
\end{tabular}

\begin{tabular}{lcc}
\hline Titolo di studio più recente (D4) & Numerosità & Percentuale \\
\hline Diploma Scuola Superiore & 1 & $7,7 \%$ \\
\hline Diploma di Laurea Triennale & 1 & $7,7 \%$ \\
\hline Diploma di Laurea Magistrale/v.o. & 5 & $38,4 \%$ \\
\hline Master I o II livello & 4 & $30,8 \%$ \\
\hline Altri diplomi & 2 & $15,4 \%$ \\
\hline
\end{tabular}


6 - Tecniche e strumenti per la gestione della relazione mentor-rifugiato

\begin{tabular}{lcc}
\hline Altri titoli di studio o professionali (D5) & Numerosità & Percentuale \\
\hline Nessuno & 4 & $30,8 \%$ \\
\hline Diploma di Laurea Magistrale/v.o. & 3 & $23,0 \%$ \\
\hline Master I o II livello & 2 & $15,4 \%$ \\
\hline Certificazioni DITALS/CEDILS & 2 & $15,4 \%$ \\
\hline Altri corsi formazione/perfezionamento & 2 & $15,4 \%$ \\
\hline & & \\
\hline Lingua madre (D6) & Numerosità & Percentuale \\
\hline Italiano & 9 & $69,2 \%$ \\
\hline Arabo & 2 & $15,4 \%$ \\
\hline Portoghese & 1 & $7,7 \%$ \\
\hline Yemba & 1 & $7,7 \%$ \\
\hline
\end{tabular}

\begin{tabular}{lcc}
\hline $\begin{array}{l}\text { Nr. lingue parlate oltre alla propria L1 } \\
\text { (D7) }\end{array}$ & Numerosità & Percentuale \\
\hline 1 & 2 & $15,4 \%$ \\
\hline 2 & 8 & $61,5 \%$ \\
\hline 3 & 2 & $15,4 \%$ \\
\hline 4 & 1 & $7,7 \%$ \\
\hline+5 & 0 & $0,0 \%$ \\
\hline
\end{tabular}

\begin{tabular}{lcc}
\hline $\begin{array}{l}\text { Lingue parlate oltre alla propria L1 } \\
\text { (D8) }\end{array}$ & Numerosità & Percentuale \\
\hline Inglese & 10 & $35,7 \%$ \\
\hline Francese & 8 & $28,6 \%$ \\
\hline Spagnolo & 4 & $14,3 \%$ \\
\hline Italiano & 4 & $14,3 \%$ \\
\hline Giapponese & 1 & $3,6 \%$ \\
\hline Cinese & 1 & $3,6 \%$ \\
\hline & Numerosità & Percentuale \\
\hline Altre esperienze (D10-14) & 8 & $61,5 \%$ \\
\hline $\begin{array}{l}\text { Ha vissuto all'estero per un tempo } \\
\text { Prolungato }\end{array}$ & 11 & $84,6 \%$ \\
\hline $\begin{array}{l}\text { Ha discusso con una persona di una } \\
\text { lingua/cultura diversa }\end{array}$ & 5 & $38,4 \%$ \\
\hline $\begin{array}{l}\text { Ha una precedente esperienza come } \\
\text { mentore in un programma formale } \\
\text { di mentorship }\end{array}$ & & \\
\hline
\end{tabular}




\begin{tabular}{lcc}
\hline $\begin{array}{l}\text { Ha una precedente esperienza in attività } \\
\text { di mentoring informale }\end{array}$ & 7 & $53,8 \%$ \\
\hline $\begin{array}{l}\text { È stato un mentee di qualcuno che ha } \\
\text { fatto da mentore }\end{array}$ & 3 & $23,1 \%$ \\
\hline
\end{tabular}

Proseguendo con l'analisi, nella parte B [tab. 3] del questionario riguardante le funzioni del mentore e del mentoring, avvalendosi di domande con scale di gradimento, è stato possibile delineare le competenze del mentor e la metodologia da adottare nei processi di mentoring.

Tabella 3 Risultati sezione 'Funzioni del mentore e del mentoring' (parte B del Questionario)

\begin{tabular}{|c|c|c|c|c|c|}
\hline È in grado di (D16-23): & $1<$ & 2 & 3 & 4 & $5>$ \\
\hline $\begin{array}{l}\text { ascoltare attivamente i suoi } \\
\text { mentee per capire i loro bisogni, } \\
\text { anche se non rispondono alle sue } \\
\text { aspettative }\end{array}$ & - & - & - & $\begin{array}{c}(8) \\
61,5 \%\end{array}$ & $\begin{array}{c}(5) \\
38,5 \%\end{array}$ \\
\hline $\begin{array}{l}\text { essere mentalmente aperto } \\
\text { a considerare i potenziali impatti } \\
\text { della transizione culturale } \\
\text { e sociale sui suoi mentee } \\
\text { (es. perdita di amici, famiglia } \\
\text { e cultura di origine, shock culturale) }\end{array}$ & - & - & $\begin{array}{c}(2) \\
15,4 \%\end{array}$ & $\begin{array}{c}(6) \\
46,1 \%\end{array}$ & $\begin{array}{c}(5) \\
38,5 \%\end{array}$ \\
\hline $\begin{array}{l}\text { essere pronto a investire } \\
\text { il suo tempo con i mentee }\end{array}$ & - & - & $\begin{array}{c}(2) \\
15,4 \%\end{array}$ & $\begin{array}{c}(5) \\
38,5 \%\end{array}$ & $\begin{array}{c}(6) \\
46,1 \%\end{array}$ \\
\hline $\begin{array}{l}\text { offrire ai suoi mentee empatia } \\
\text { umana e compassione }\end{array}$ & $\begin{array}{c}(1) \\
7,7 \%\end{array}$ & - & - & $\begin{array}{c}(4) \\
30,8 \%\end{array}$ & $\begin{array}{c}(8) \\
61,5 \%\end{array}$ \\
\hline $\begin{array}{l}\text { accettare di essere monitorato } \\
\text { da un professionista durante } \\
\text { la sua attività di mentore }\end{array}$ & - & $\begin{array}{c}(1) \\
7,7 \%\end{array}$ & $\begin{array}{c}(1) \\
7,7 \%\end{array}$ & $\begin{array}{c}(5) \\
38,5 \%\end{array}$ & $\begin{array}{c}(6) \\
46,1 \%\end{array}$ \\
\hline $\begin{array}{l}\text { accrescere la sua conoscenza delle } \\
\text { lingue e delle culture dei mentee }\end{array}$ & - & - & $\begin{array}{c}(2) \\
15,4 \%\end{array}$ & $\begin{array}{c}(5) \\
38,5 \%\end{array}$ & $\begin{array}{c}(6) \\
46,1 \%\end{array}$ \\
\hline $\begin{array}{l}\text { aiutare i mentee ad affrontare } \\
\text { le difficoltà della ricerca del lavoro }\end{array}$ & - & - & $\begin{array}{c}(4) \\
30,8 \%\end{array}$ & $\begin{array}{c}(2) \\
15,4 \%\end{array}$ & $\begin{array}{c}(7) \\
53,8 \%\end{array}$ \\
\hline $\begin{array}{l}\text { utilizzare strumenti digitali } \\
\text { (computer, tablet, telefono } \\
\text { cellulare ecc.) per aiutare i mentee }\end{array}$ & - & $\begin{array}{c}(1) \\
7,7 \%\end{array}$ & $\begin{array}{c}(4) \\
30,8 \%\end{array}$ & $\begin{array}{c}(1) \\
7,7 \%\end{array}$ & $\begin{array}{c}(7) \\
53,8 \%\end{array}$ \\
\hline
\end{tabular}


Monaci

6 - Tecniche e strumenti per la gestione della relazione mentor-rifugiato

\begin{tabular}{|c|c|c|c|c|c|}
\hline $\begin{array}{l}\text { Secondo lei, quali qualità } \\
\text { dovrebbe avere un buon } \\
\text { mentore? (D25-32) }\end{array}$ & $1<$ & 2 & 3 & 4 & $5>$ \\
\hline costruire relazioni & - & - & - & $\begin{array}{c}(3) \\
23,1 \%\end{array}$ & $\begin{array}{c}(10) \\
76,9 \% \\
\end{array}$ \\
\hline ascoltare & - & - & - & $\begin{array}{c}(1) \\
7,7 \%\end{array}$ & $\begin{array}{c}(12) \\
92,3 \%\end{array}$ \\
\hline gestire i conflitti & - & $\begin{array}{c}(1) \\
7,7 \%\end{array}$ & - & $\begin{array}{c}(7) \\
53,8 \%\end{array}$ & $\begin{array}{c}(5) \\
38,5 \%\end{array}$ \\
\hline $\begin{array}{l}\text { coaching (facilitare/accompagnare } \\
\text { verso l'obiettivo) }\end{array}$ & - & $\begin{array}{c}(1) \\
7,7 \%\end{array}$ & - & $\begin{array}{c}(6) \\
46,1 \%\end{array}$ & $\begin{array}{c}(6) \\
46,1 \%\end{array}$ \\
\hline promuovere l'auto-riflessione & - & - & $\begin{array}{c}(1) \\
7,7 \%\end{array}$ & $\begin{array}{c}(5) \\
38,5 \%\end{array}$ & $\begin{array}{c}(7) \\
53,8 \%\end{array}$ \\
\hline incoraggiare & - & - & $\begin{array}{c}(3) \\
23,1 \%\end{array}$ & $\begin{array}{c}(4) \\
30,8 \%\end{array}$ & $\begin{array}{c}(6) \\
46,1 \%\end{array}$ \\
\hline fornire e ricevere feedback & - & - & $\begin{array}{c}(3) \\
23,1 \%\end{array}$ & $\begin{array}{c}(3) \\
23,1 \%\end{array}$ & $\begin{array}{c}(7) \\
53,8 \%\end{array}$ \\
\hline $\begin{array}{l}\text { aiutare i mentee a sviluppare } \\
\text { capacità di risoluzione dei problemi }\end{array}$ & $\begin{array}{c}(1) \\
7,7 \%\end{array}$ & - & - & $\begin{array}{c}(2) \\
15,4 \%\end{array}$ & $\begin{array}{c}(10) \\
76,9 \%\end{array}$ \\
\hline $\begin{array}{l}\text { Secondo lei, per un mentore quali } \\
\text { sono gli Aspetti più importanti } \\
\text { del tutoraggio? (D33-40) }\end{array}$ & $1<$ & 2 & 3 & 4 & $5>$ \\
\hline $\begin{array}{l}\text { Entrare in contatto con persone } \\
\text { di culture diverse }\end{array}$ & - & $\begin{array}{c}(1) \\
7,7 \%\end{array}$ & $\begin{array}{c}(1) \\
7,7 \%\end{array}$ & $\begin{array}{c}(5) \\
38,5 \%\end{array}$ & $\begin{array}{c}(6) \\
46,1 \%\end{array}$ \\
\hline $\begin{array}{l}\text { Sviluppare abilità di career } \\
\text { coaching (specializzazione } \\
\text { per la consulenza) }\end{array}$ & - & - & $\begin{array}{c}(6) \\
46,1 \%\end{array}$ & $\begin{array}{c}(3) \\
23,1 \%\end{array}$ & $\begin{array}{c}(4) \\
30,8 \%\end{array}$ \\
\hline $\begin{array}{l}\text { Ricevere formazione per lo } \\
\text { sviluppo personale e professionale }\end{array}$ & - & - & $\begin{array}{c}(3) \\
23,1 \%\end{array}$ & $\begin{array}{c}6 \\
46,1 \%\end{array}$ & $\begin{array}{c}4 \\
30,8 \%\end{array}$ \\
\hline $\begin{array}{l}\text { Rafforzare le capacità di } \\
\text { leadership e Coaching }\end{array}$ & - & - & $\begin{array}{c}(4) \\
30,8 \%\end{array}$ & $\begin{array}{c}(7) \\
53,8 \%\end{array}$ & $\begin{array}{c}(2) \\
15,4 \%\end{array}$ \\
\hline $\begin{array}{l}\text { Migliorare la consapevolezza } \\
\text { interculturale (es. comprendere } \\
\text { i problemi e gli ostacoli incontrati } \\
\text { dai mentee nell'impatto con } \\
\text { il Paese di arrivo). }\end{array}$ & - & - & $\begin{array}{c}(1) \\
7,7 \%\end{array}$ & $\begin{array}{c}(4) \\
30,8 \%\end{array}$ & $\begin{array}{c}(8) \\
61,5 \%\end{array}$ \\
\hline $\begin{array}{l}\text { Ampliare le capacità } \\
\text { di comunicazione }\end{array}$ & - & - & $\begin{array}{c}(1) \\
7,7 \%\end{array}$ & $\begin{array}{c}(6) \\
46,1 \%\end{array}$ & $\begin{array}{c}(6) \\
46,1 \%\end{array}$ \\
\hline $\begin{array}{l}\text { Acquisire una prospettiva } \\
\text { internazionale del proprio campo } \\
\text { o occupazione }\end{array}$ & - & - & $\begin{array}{c}(1) \\
7,7 \%\end{array}$ & $\begin{array}{c}(7) \\
53,8 \%\end{array}$ & $\begin{array}{c}(5) \\
38,5 \%\end{array}$ \\
\hline $\begin{array}{l}\text { Condividere preziose conoscenze } \\
\text { acquisite con la propria esperienza }\end{array}$ & - & - & $\begin{array}{c}(2) \\
15,4 \%\end{array}$ & $\begin{array}{c}7 \\
53,8 \%\end{array}$ & $\begin{array}{c}4 \\
30,8 \%\end{array}$ \\
\hline
\end{tabular}




\begin{tabular}{lcc}
\hline $\begin{array}{l}\text { Tipi di mentoring ritenuti più } \\
\text { adatti per futuri mentee (D41) }\end{array}$ & Preferenze & Percentuale \\
\hline Tutoraggio one-to-one & 7 & $35,0 \%$ \\
\hline Mentoring di gruppo & 5 & $25,0 \%$ \\
\hline Mentoring a distanza & 1 & $5,0 \%$ \\
\hline Modalità combinate & 7 & $35,0 \%$ \\
\hline & Numerosità & Percentuale \\
\hline Importanza di incontrare & & $84,6 \%$ \\
\hline i mentee di persona (D42) & 11 & - \\
\hline Sì, sempre & - & $15,4 \%$ \\
\hline No & 2 & - \\
\hline A volte & - & \\
\hline Solo all'inizio & & \\
\hline
\end{tabular}

Infine, altri dati molto interessanti raccolti, relativi alla parte D del questionario [tab. 4], sono stati tratti da riflessioni guidate su gli aspetti intrinsechi al ruolo del mentor.

Tabella 4 Risultati sezione 'Riflessioni riguardanti le relazioni con gli altri' (parte D del Questionario)

\begin{tabular}{lccccc}
\hline Riflessioni (D53-55) & $\mathbf{1}$ & $\mathbf{2}$ & $\mathbf{3}$ & $\mathbf{4}$ & $\mathbf{5}>$ \\
\hline $\begin{array}{l}\text { Sono disposto ad accettare } \\
\text { che il mentee possa Insegnarmi } \\
\text { delle cose }\end{array}$ & - & - & - & $(4)$ & $(9)$ \\
\hline $\begin{array}{l}\text { So in quali aspetti la sua cultura } \\
\text { e quella del Mentee differiscono } \\
\text { profondamente }\end{array}$ & - & - & $(2)$ & $(9)$ & $(2)$ \\
\hline $\begin{array}{l}\text { Sono pronto ad accettare che il } \\
\text { mio mentee Abbia comportamenti }\end{array}$ & - & - & - & $(4)$ & $(9)$ \\
$\begin{array}{l}\text { verbali e non verbali Diversi da } \\
\text { quelli della mia cultura di origine }\end{array}$ & & & & $30,8 \%$ & $69,2 \%$ \\
\hline & & & & & $69,2 \%$ \\
\hline $\begin{array}{l}\text { Riflessioni (D56-61) } \\
\text { Sospendere il giudizio sul mentee }\end{array}$ & $(1)$ & - & $(4)$ & $(5)$ & $(3)$ \\
e sulla sua cultura & $7,7 \%$ & & $30,8 \%$ & $38,5 \%$ & $23,1 \%$ \\
\hline $\begin{array}{l}\text { Entrare in empatia, mettersi } \\
\text { nei panni del suo mentee }\end{array}$ & $(1)$ & $(1)$ & $(1)$ & $(7)$ & $(3)$ \\
\hline $\begin{array}{l}\text { Ascoltare attentamente } \\
\text { e attivamente }\end{array}$ & - & - & $(1)$ & $(5)$ & $(7)$ \\
\hline & & & $7,7 \%$ & $38,5 \%$ & $53,8 \%$ \\
\hline
\end{tabular}




\begin{tabular}{|c|c|c|c|c|c|}
\hline $\begin{array}{l}\text { Relativizzare i suoi valori, } \\
\text { convinzioni, comportamenti }\end{array}$ & - & - & $\begin{array}{c}(3) \\
23,1 \%\end{array}$ & $\begin{array}{c}(7) \\
53,8 \%\end{array}$ & $\begin{array}{c}(3) \\
23,1 \%\end{array}$ \\
\hline $\begin{array}{l}\text { Decentralizzarsi dal suo ruolo } \\
\text { sociale, dal suo comportamento } \\
\text { abituale }\end{array}$ & - & - & $\begin{array}{c}(5) \\
38,5 \%\end{array}$ & $\begin{array}{c}(6) \\
46,1 \%\end{array}$ & $\begin{array}{c}(2) \\
15,4 \%\end{array}$ \\
\hline $\begin{array}{l}\text { Discutere in modo costruttivo } \\
\text { con il mentee se per esempio un } \\
\text { atteggiamento le sembra offensivo }\end{array}$ & - & - & $\begin{array}{c}(2) \\
15,4 \%\end{array}$ & $\begin{array}{c}(6) \\
46,1 \%\end{array}$ & $\begin{array}{c}(5) \\
38,5 \%\end{array}$ \\
\hline
\end{tabular}

In conclusione, visto che genericamente i programmi di mentorship durano per mesi, è di fondamentale importanza tenere traccia dello stato di avanzamento e dei relativi progressi, per effettuare eventuali aggiustamenti e riadattare la struttura del programma quando necessario, monitorare la pertinenza delle attività rispetto ai bisogni dei beneficiari e al contesto in continua evoluzione. Per queste ragioni sarebbe preferibile affiancare alla figura del mentor dei professionisti per coordinare le attività e dare un supporto durante lo svolgimento del corso.

L'indagine sulla percezione del valore e dell'impatto del programma viene solitamente effettuata attraverso la registrazione di opinioni e commenti dei partecipanti pre e post esperienza di mentorship, in modo tale da confrontare i risultati iniziali con quelli finali anziché optare per una valutazione esclusivamente finale con il rischio di sprecare potenziali benefici dell'intervento. Fornendo quindi un supporto su misura e mirato per l'integrazione, valutando la qualità della relazione fra il mentors e i mentees, questi strumenti diventano un fattore decisivo per il successo del programma.

Infine, se è vero che la valutazione dei programmi di mentorship si concentra principalmente sulla valutazione del criterio dell'efficacia, sarebbe utile documentare anche la progettazione e realizzazione delle attività in vista della possibilità di replicabilità. Questa disamina, per capire come progettare e condurre programmi di mentorship di successo nel futuro. 


\section{Bibliografia}

Ahad, A.; Le Coz, C.; Beirens, H. (2020). Using Evidence to Improve Refugee Resettlement: A Monitoring and Evaluation Road Map. Brussels: Migration Policy Institute Europe. https://www.migrationpolicy.org/research/ refugee-resettlement-monitoring-evaluation-road-map.

Ahmad, K.S. et al. (2015). «Learning English Vocabulary In A Mobile Assisted Language Learning (MALL) Environment: A Sociocultural Study Of Migrant Women». Interdisciplinary Journal of E-skills and Life Long Learning, $11,25-45$.

ALNAP (2016). Evaluation of Humanitarian Action Guide. London: ALNAP/ODI. https://www.alnap.org/help-library/evaluation-of-humanitarian-action-guide.

Aloisi, E.; Perna, A. (2019). Ataya. Manuale multilivello per adulticon bassa o nulla scolarità pregressa. Ranica: Sestante.

Altherr Flores, J. (2017). «Social Semiotics and Multimodal Assessment of L2 Emergent Readers from Refugee Backgrounds». Sosinski, M. (ed.), Alfabetización y apredizaje de idiomas por adultos: investigación, politica educativa y práctica docente. Granada: Editorial Universidad de Granada, 9-32.

Amato, A.; Gavioli, L. (2008). Il ruolo dell'interprete-mediatore nella comunicazione istituzionale medico-paziente: un'analisi dei contributi non traduttivi. Perugia: Guerra, 293-319.

Ambrosini, M. (2011). Sociologia delle migrazioni. Bologna: il Mulino.

Amoruso, C. (2010). In parole semplici. La riscrittura funzionale dei testi nella classe plurilingue. Palermo: Palumbo.

Anfosso, G.; Polimeni, G.; Salvadori, E. (a cura di) (2016). Le autobiografie linguistiche tra teoria e didattica. Milano: FrancoAngeli.

Angelini, E. (2021). «Implicazioni psico-affettive e socioculturali nell'insegnamento della L2 a richiedenti asilo». Caruana et al. 2021, 51-62. http://doi. org/10.30687/978-88-6969-501-8/004.

Arcuri, A.; Mocciaro, E. (2016). «ll profilo professionale del docente di italiano L2 per utenze fragili». De Marco, A. (a cura di), Lingue al plurale: la formazione degli insegnanti. Perugia: Guerra, 341-50. 
Bagna, C. et al. (2003). «Italiano e lingue immigrate: verso un plurilinguismo consapevole o verso varietà di contatto?». Valentini, A. et al. (a cura di), Ecologia linguistica. Roma: Bulzoni, 201-22.

Bagna, C. et al. (2004). Toscane favelle. Lingue immigrate nella provincia di Siena. Perugia: Guerra.

Bagna, C.; Gallina, F.; Machetti, S. (2018). «L'approccio del Linguistic Landscape applicato alla didattica dell'italiano L2 per studenti internazionali». Coonan, C.M.; Bier, A.; Ballarin, E. (a cura di), La didattica delle lingue nel nuovo millennio. Le sfide dell'internazionalizzazione. Venezia: Edizioni Ca' Foscari, 219-32. http://doi.org/10.30687/978-88-6969-227-7/014.

Balboni, P.E. (1999). Parole comuni, culture diverse: guida alla comunicazione interculturale. Venezia: Marsilio.

Balboni, P.E. (2007). La comunicazione interculturale. Venezia: Marsilio.

Balboni, P.E. (2014). Didattica dell'italiano come lingua seconda e straniera. Torino: Bonacci-Loescher.

Balboni, P.E.; Caon, F. (2015). La comunicazione interculturale. Venezia: Marsilio.

BAMF, Bundesamt für Migration und Flüchtlinge (2015). Konzept für einen bundesweiten Alphabetisierungskurs. Überarbeitete Neuauflage. Mai 2015. Nürnberg: Bundesamt für Migration und Flüchtlinge. https://www. bamf. de/SharedDocs/Anlagen/DE/Integration/Integrationskurse/ Kurstraeger/KonzepteLeitfaeden/konz-f-bundesw-ik-mitalphabet.pdf?__blob=publicationFile.

Banfi, E. et al. (a cura di) (2006). Fenomeni di mediazione interlinguistica e interculturale. Perugia: Guerra.

Baraldi, C.; Gavioli, L. (2008). «Cultural Presuppositions and Re-Contextualization of Medical Systems in Interpreter-Mediated Interactions». Curare: Journal of Medical Anthropology, 31(2/3), 193-204.

Baraldi, C.; Gavioli, L. (2010). «Interpreter-Mediated Interaction as a Way to Promote Multilingualism». Meyer, B.; Apfelbaum, B. (eds), Multilingualism at Work. Amsterdam; Philadelphia: John Benjamins, 141-62.

Beacco, J.-C. (2016). Ėcole et politiques linguistiques. Pour une gestion de la diversité linguistique. Paris: Didier.

Bennett, M.J. (1993). «Toward Ethnorelativism: A Developmental Model of Intercultural Sensitivity». Paige, M.R. (ed.), Education for the intercultural experience. Yarmouth (ME): Intercultural Press, 21-71.

Bennett, M.J. (2015). Principi di comunicazione interculturale. Paradigmi e pratiche. Milano: FrancoAngeli.

Benucci, A. (2001). «La competenza interculturale». Diadori, P. (a cura di), Insegnare italiano a stranieri. Firenze: Le Monnier, 32-43.

Benucci, A. (2005). Le lingue romanze. Torino: UTET.

Benucci, A. (a cura di) (2007a). Italiano libera-mente. L'insegnamento dell'italiano a stranieri in carcere. Perugia: Guerra.

Benucci, A. (2007b). «Du côté de l'apprenant: processus cognitifs et stratégies personnels». Capucho, F. et al. (eds), Diálogos em Intercompreensão, vol. 1. Lisboa: Universidade Catolica, 149-57.

Benucci, A. (a cura di) (2008). Contenuti, metodi e approcci per insegnare italiano a stranieri. Percorsi di formazione. Perugia: Guerra.

Benucci, A. (a cura di) (2009). Liberare la comunicazione. Perugia: Guerra.

Benucci, A. (2013). La formazione agli aspetti culturali e interculturali. Benucci, A. (a cura di), Formazione e pratiche didattiche in italiano L2. Perugia: Guerra, 13-34. 
Benucci, A. (2014). Italiano L2 e interazioni professionali. Torino: UTET Università. Benucci, A. (2015). «Intercultural and Inter-linguistic Models in Disadvantaged Migration Contexts». Argondizzo, C. (ed.), European Projects in University Language Centres. Bern: Peter Lang, 81-104.

Benucci, A. (2017). «Répertoires linguistiques des détenus étrangers en Italie et en Europe: premiers résultats des projets RiUscire et DEPORT». Béacco, J.-C. et al. (eds), The Linguistic Integration of Adult Migrants / L'intégration linguistique des migrants adultes. Some Lessons from Research / Les enseignements de la recherche. Berlin: De Gruyter Mouton, 147-52.

Benucci, A. (2018). «Competenze interculturali e interculturalità». Santipolo, M.; Mazzotta, P., L'educazione linguistica oggi. Torino: UTET Università, 189-93.

Benucci, A. (2020). «Quale didattica per il plurilinguismo oggi?». EL.LE, 9(2),

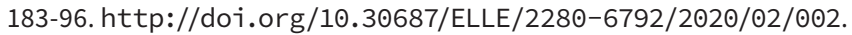

Benucci, A. (2021). «Inclusione, contesti svantaggiati e Linguistica educativa». Benucci et al. 2021, 9-25.

Benucci, A. et al. (2010). L'ora d'italiano. Perugia: Guerra.

Benucci, A. et al. (2021). Didattica inclusiva e azioni educative in contesti di vulnerabilità. Roma: Aracne.

Benucci, A.; Biotti, F. (2007). «Aspetti culturali». Benucci, A. (a cura di), Sillabo di italiano per stranieri. Una proposta del Centro Linguistico dell'Università per Stranieri di Siena. Perugia: Guerra, 187-95, 205-8.

Benucci, A.; Birello, M. (2017). «Immigrants and Prisons: Good Practices in Europe». Béacco, J.-C. et al. (eds), The Linguistic Integration of Adult Migrants / L'intégration linguistique des migrants adultes. Some Lessons from Research / Les enseignements de la recherche. Berlin: De Gruyter Mouton, 371-6.

Benucci, A.; Cortés Velásquez, D. (2011). «Intercomprensione e apprendimento: abilità parziali e processi cognitivi». Meissner, F.-J. et al. (eds), Intercomprehension: Learning, Teaching, Research. Apprentissage, enseignement, recherche. Lernen, Leheren, Forschung. Tübingen: Narr Verlag, 319-36.

Benucci, A.; Grosso, G.I. (2012). «L'italiano in carcere: approcci di riferimento e strumenti operativi». Grassi, R. (a cura di), Nuovi contesti d'acquisizione e insegnamento: l'italiano nelle realtà plurilinguistiche. Perugia: Guerra, 89-100.

Benucci, A.; Grosso, G.I. (2015). Plurilinguismo, contatto e superdiversità nel contesto penitenziario italiano. Pacini: Pisa.

Benucci, A.; Grosso, G.I. (2017). Buone pratiche e repertori linguistici in carcere. Roma: Aracne Editrice.

Benucci, A.; Grosso, G.I. (2021). «Aspetti interculturali nella comunicazione medico paziente: il punto di vista del personale sanitario e dei pazienti». Caruana et al. 2021, 105-13. http://doi.org/10.30687/978-88-69695०1-8/००9.

Benucci, A.; Maiorano, P.B. (a cura di) (2015). L'ABC del lavoratore. Italiano L2: apprendimento linguistico e professionale. Siena: Tipografia Senese Editrice.

Benvenuti, P. (2006). «La Convenzione di Ginevra sullo status dei rifugiati». Pinechi, L. (a cura di), La tutela internazionale dei diritti umani. Norme, garanzie, prassi. Milano: Giuffrè, 151-72.

Berthoz, A. (2015). «Empathie: un regard scientifique sur les bases de la relation à l'aautre'». Diasporiques, 29, 24-9.

Bettoni, C. (2006). Usare un'altra lingua. Guida alla pragmatica interculturale. Bari: Laterza. 
Bhugra, D. (2004). «Migration and Mental Health». Acta Psychiatrica Scandinavica Journal, 109(4), 243-58.

Bialystok, E. (1986). «Factors in the Growth of Linguistic Awareness». Child Development, 57(2), 498-510.

Bialystok, E. (2001). Bilingualism in Development: Language, Literacy and Cognition. New York: Cambridge University Press.

Bintinger, G.; Eichelberger, H.; Wilhelm, M. (2005). «Von der Integration zur Inklusion». Grubich, R. (ed.), Inklusive Pädagogik. Beiträge zu einem anderen Verständnis von Integration. Wien; Meran: Innsalz, Aspach, 20-42.

Bittman, T. (2011). Mentoring for Migrants. Labour Market Integration of Migrants in Austria: An Example of a Significant and Effective Integration Model [Master theses]. Nice: Centre International de Formation Européenne, Institut Européen Des Hautes Études Internationales.

Blinder, S. (2015). «Imagined Immigration: The Impact of Different Meanings of 'Immigrants' in Public Opinion and Policy Debates in Britain». Political Studies, 63(1), 80-100.

Bond, S. (2010). Women on the Move. Evaluating a Refugee Mentoring Pilot Project. Fitzroy: Brotherhood of St Laurence. https://library.bsl.org. au/jspui/bitstream/1/5993/1/Bond_Women_on_the_Move_evaluation_2010.pdf.

Borg, J. (2009). Il linguaggio del corpo: guida all'interpretazione del linguaggio non verbale. Milano: Tecniche Nuove.

Borghetti, C. (2016). Educazione linguistica interculturale: origini, modelli, sviluppi recenti. Cesena; Bologna: Caissa Italia.

Borghetti, C. (2018). «Studiare l'apprendimento linguistico interculturale come pratica discorsiva e interazionale». Coonan, C.M.; Bier, A.; Ballarin, E. (a cura di), La didattica delle lingue nel nuovo millennio. Le sfide dell'internazionalizzazione. Venezia: Edizioni Ca' Foscari, 413-28. http://doi. org/10.30687/978-88-6969-227-7/025.

Bormioli, A. (a cura di) (2017). Sistema penitenziario e detenuti stranieri. Roma: Aracne Editrice.

Borrelli, E. (2020). «ll rapporto tra genere e migrazioni: come cambiano le opportunità per donne e uomini migranti» Progeu, 2 ottobre. https:// tinyurl.com/yea23bzf.

Borri, A. (2019). A piccoli passi. Alfabetizzazione e competenze di base. Torino: Loescher.

Borri, A. et al. (2014). Italiano L2 in contesti migratori. Sillabo e descrittori dall'alfabetizzazione all'A1. Torino: Loescher.

Borri, A. et al. (2016). Pari e dispari. Italiano L2 per adulti in classi ad abilità differenziate. Torino: Loescher.

Bortolon Guidolin, G. (2019). «Diversificare la didattica: il compito stratificato, differenziato e aperto con studenti analfabeti e bassamente scolarizzati». Caon, F.; Brichese, A. (a cura di), Insegnare italiano ad analfabeti. Torino: Bonacci; Loescher, 131-8.

Boswell, C.; Geddes, A.; Scholten, P. (2011). «The Role of Narratives in Migration Policy-Making: A Research Framework». The British Journal of Politics and International Relations, 13(1), 1-11.

Bourque, F.; Van Der Ven, E.; Malla, A. (2011). «A Meta-Analysis of the Risk for Psychotic Disorders Among First- and Second-Generation Immigrants». Psychological Medicine, 41, 897-910. 
Bretegnier, A. (2002). «Introduction. Regards sur l'insécurité linguistique». Bretegnier, A.; Ledegen, G. (éds), Sécurité/insécurité linguistique: terrains et approches diversifiés. Propositions théoriques et méthodologiques. Paris: L'Harmattan, 7-33.

Brichese, A. (2019). «Dalla parte dello studente: caratteristiche dello studente analfabeta e bassamente scolarizzato». Caon, F.; Brichese, A. (a cura di), Insegnare italiano ad analfabeti. Torino: Bonacci-Loescher, 12-22.

Brichese, A.; Tonioli, V. (2017). Il mediatore interlinguistico e interculturale e il facilitatore linguistico. Natura e competenze. Venezia: Marsilio.

Browder, C. (2018). «Recently Resettled Refugee Students Learning English in Us High Schools: The Impact of Students' Educational Background». Shapiro et al. 2018, 17-32.

Bruner, J. (1991). «The Narrative Construction of Reality». Critical Inquiry, 18, 1-21.

Byram, M. (1997). Teaching and Assessing Intercultural Communicative Competence. Bristol: Multilingual Matters.

Byram, M. (1999). «Apprendre une langue, apprendre des cultures». Gauchola, R. et al. (éds), Repères et Applications en didactique des langues. Barcelona: Universitat Autònoma de Barcelona, 121-8.

Caddéo, S.; Jamet, M.C. (2013). L'intercompréhension: une autre approche pour l'enseignement des langues. Paris: Hachette.

Calvet, L.-J. (2002). Le marché aux langues: essai de politologie linguistique sur la mondialisation. Paris: Plon.

Calvet, L.-J. (1998). «L'insécurité linguistique et les situations africaines». Calvet, L.-J.; Moreau, M.L. (éds), Une ou des normes? Insécurité linguistique et normes endogènes en Afrique francophone. Paris: Agence de francophonie, 7-38.

Canagarajah, S. (2013). Translingual Practice: Global Englishes and Cosmopolitan Relations. London: Routledge.

Canagarajah, S.; Liyanage, I. (2012). «Lessons from Pre-Colonial Multilingualism». Martin-Jones, M.; Blackledge, A.; Creese, A (eds), The Routledge Handbook of Multilingualism. London: Routledge, 49-65.

Cantor-Grae, E.; Selten, J.-P. (2005). «Schizophrenia and Migration: A MetaAnalysis and Review». The American Journal of Psychiatry, 162(1), 12-24. https://doi.org/10.1176/appi.ajp.162.1.12.

Caon, F. (2008a). Educazione linguistica e differenziazione: gestire eccellenza e difficoltà. Torino: UTET Università.

Caon, F. (2008b). «Una glottodidattica per classi ad abilità differenziate: teorie di riferimento e proposte operative». Caon, F. (a cura di), Insegnare italiano nella Classe ad Abilità Differenziate. Perugia: Guerra, 11-61.

Caon, F. (a cura di) (2016). Educazione linguistica nella classe ad abilità differenziate. Torino: Bonacci-Loescher.

Caon, F. (2019). «Dalla parte del docente: didattica dell'italiano L2 a studenti analfabeti e bassamente scolarizzati». Caon, F.; Brichese, A. (a cura di), Insegnare italiano ad analfabeti. Torino: Bonacci-Loescher, 23-32.

Caon, F.; Tonioli, V. (2016). «La sfida delle classi ad abilità linguistiche differenziate». SeLM, 7-9, 137-54.

Capucho, F. et al. (2007). Diálogos em Intercompreensão. Lisboa: Universidade Católica.

Carbonara, V.; Scibetta, A. (2018). «ll translanguaging come strumento efficace per la gestione delle classi plurilingui: il progetto L'altroparlante». RILA, 1,65-83. 
Caritas; Migrantes (2020). XXIX Rapporto Immigrazione 2020. Conoscere per comprendere. Todi: Tau. https://www.migrantes.it/wp-content/uploads/sites/50/2020/10/RICM_2020_DEF.pdf.

Carroll, J.B. (1964). Language and Thought. Englewood-Cliffs (NJ): PrenticeHall.

Caruana, S.; Chircop, K.; Gauci, P.; Pace, M. (a cura di) (2021). Politiche e pratiche per l'educazione linguistica, il multilinguismo e la comunicazione interculturale. Edizioni Ca' Foscari: Venezia. http://doi.org/10.30687/97888-6969-501-8.

Casadei, S.; Franceschetti, M. (a cura di) (2009). Il mediatore culturale in sei Paesi europei: (Italia, Francia, Germania, Grecia, Regno Unito, Spagna): ambiti di intervento, percorsi di accesso e competenze. Roma: ISFOL.

Casi, P. (2015). «ll diritto al codice rosso per gli analfabeti: le anomalie italiane nell'istruzione degli adulti». Insegno Italiano L2 in classe, 1, 65-70.

Cassandro, M.; Maffei, S. (2007). «Didattica per progetti». Benucci, A. (a cura di), Sillabo di italiano per stranieri. Perugia: Guerra, 233-70.

Castles, S. et al. (2020). The Age of Migrations: International Population Movements in the Modern World. 6th ed. New York: The Guilford Press.

Catarci, M.; Fiorucci, M. (a cura di) (2014). Orientamenti interculturali per la cittadinanza. Roma: Armando.

Cattedra, R. (2014). «Metamorfosi urbane. Progetti, pratiche e ri-usi della città contemporanea». Memoli, M.; Governa, F. (a cura di), Geografia dell'urbano. Spazi, politiche, pratiche della città. Roma: Carocci, 249-76.

Cavagnoli, S. (2014). «L'autobiografia linguistica a scuola tra plurilinguismo e affettività». Landolfi, L. (eds), Crossroads Languages in Emotion. Napoli: Photocity University Press, 179-88.

Centro Come (2001). Anche le mamme a scuola. Vademecum per la realizzazione di corsi di alfabetizzazione in italiano rivolti a madri immigrate. http:// www.centrocome.it/come_files/userfiles/File/AreaProgetti/ ANCHE_LE_MAMME_A_SCUOLA.PDF

Chasikou, F.; Ypsilandis, G.S. (2018). «Considering the Use of Mobile Phone by Refugees in Greek Language Learning». TISLID2018: Digital Lives and Cultures. http://www.tislid18.ugent.be/.

Chermak, S.M. (1995). Victims in the New: Crime and the American News Media. Boulder (CO): Westview.

Choi, J.; Najar, U. (2017). «Immigrant and Refugee Women's Resourcefulness in English Language Classrooms: Emerging Possibilities Through Plurilingualism». Literacy and Numeracy Studies: An International Journal in the Education and Training of Adults, 25(1), 20-37.

Clark, C.; Dugdale, G. (2008). Literacy Changes Lives: The Role of Literacy in Offending Behavior. London: National Literacy Trust.

Codagnone, C.; Kluzer, S. (2011). «ICT for the Social and Economic Integration of Migrants into Europe». http://ftp.jrc.es/EURdoc/JRC63183.

Cognigni, E. (2013). «Italiano lingua d'altre": L2 e racconto di sé in prospettiva di genere». PREMIA. Processo di Empowerment per Immigrati Analfabeti. Porto Sant'Elpidio: Wizarts Editore, 63-82.

Cognigni, E. (2014). «Migrazione femminile e bisogni di apprendimento in italiano $L 2$. Uno studio di caso presso le donne del ricongiungimento familiare». EL.LE, 3(3), 465-81. http://doi.org/10.14277/2280-6792/117p.

Condelli, L. et al. (2008). «What Works for Adult Literacy Students of English as a Second Language». Reder, S.; Bynner, J. (eds), Tracking Adult Litera- 
cy and Numeracy: Longitudinal Studies of Adult Education. New York: Routledge, 59-84.

Conklin, L. (2012). What's next?: A Multilevel Phonics Approach for ESL Students. Syracuse (NY:) New Readers Press.

Coonan, C.M. (2020). La metodologia CLIL. Seminario Education \& Training 2020: pratica CLIL e strategie cooperative. Campobasso: Ufficio Scolastico Regionale per il Molise.

Corino, E. (2014). «Didattica delle lingue corpus-based». EL.LE, 3(2), 231-58. http://doi.org/10.14277/2280-6792/99p.

Cortés Velásquez, D.; Nuzzo, E. (2017). «Disdire un appuntamento: spunti per la didattica dell'italiano L2 a partire da un corpus di parlanti nativi». Italiano LinguaDue, 1, 17-36.

Cortés Velásquez, D.; Nuzzo, E. (2018). «Un'indagine sulla consapevolezza metapragmatica di parlanti plurilingui». De Meo, A.; Rasulo, M. (a cura di), Usare le lingue seconde. Comunicazione, tecnologia, disabilità, insegnamento. Milano: OfficinaVentuno/AltLa, 99-115.

Cortés Velásquez, D.; Faone, S.; Nuzzo, E. (2017). «Analizzare i manuali per l'insegnamento delle lingue: strumenti per una glottodidattica applicata». Italiano LinguaDue, 9, 1-74. https://doi.org/10.13130/2037-3597/9871.

CRELL (2006). Measuring Active Citizenship in Europe. European Commission, Directorate-General Joint Research Centre, Institute for the Protection and Security of the Citizen.

Crepet, A. et. al. (2017). «Mental Health and Trauma in Asylum Seekers Landing in Sicily in 2015: A Descriptive Study of Neglected Invisible Wounds». Confl Health, 11, 1.

Cummins, J. (1979). Cognitive/Academic Language Proficiency, Linguistic Interdependence, the Optimum Age Question and Some Other Matters. Toronto: Ontario Institute for Studies in Education, 121-9. Working Papers on Bilingualism 19.

Cummins, J. (2001). «Bilingual Children's Mother Tongue: Why Is It Important for Education?». Sprogforum, 19(7), 15-20.

Cummins, J. (2005). «Teaching for Cross-Language Transfer in Dual Language Education: Possibilities and Pitfalls». TESOL Symposium on Dual Language Education: Teaching and Learning Two Languages in the EFL Setting, 1-17.

D'Agostino, M. (2017). «Analfabeti nell'Italia di ieri e di oggi. Dati, modelli, persone, parole. La lezione di Tullio De Mauro». Bollettino del Centro di studi filologici e linguistici siciliani, 28, 35-58.

D'Agostino, M.; Sorce, G. (a cura di) (2016). Nuovi migranti e nuova didattica. Esperienze al CPIA Palermo 1. Palermo: Scuola di Lingua Italiana per Stranieri, Università di Palermo.

D’Annunzio, B.; Della Puppa, F. (2006). «Un modello operativo: l'unità differenziata stratificata». Caon, F. (a cura di), Insegnare italiano nelle classi ad abilità differenziate. Perugia: Guerra, 138-55.

Da Pra, L.M.G. (2021). «Profilo e ruolo del formatore nell'ambito delle attività pre-partenza». Benucci et al. 2021, 83-112.

Damiani, V.; Agrusti, G. (2019). «Promuovere l'inclusione di migranti e rifugiati attraverso risorse e-learning e attività in presenza: un'esperienza sul campo in Italia». Italian Journal of Educational Technology, 28(3), 210-26. https://doi.org/10.17471/2499-4324/1144

Danon, M.; Miltenburg, A. (2001). Rifugiati politici e salute mentale. Padova: Offsetinvicta. 
De Beni, R.; Moè, A. (2000). Motivazione e apprendimento. Bologna: il Mulino.

De Carlo, M. (2011) Intercomprensione e educazione al plurilinguismo. Porto Sant'Elpidio: Wizarts Editore.

De Fina, A.; Baynham, M. (2006). Dislocations / Relocations. Narratives of Displacement. London: Routledge.

De Fina, A.; Georgakopoulou, A. (2021). Analyzing Narrative: Discourse and Sociolinguistic Perspectives. Cambridge (MA): Cambridge University Press.

De Marco, Anna (2021). «Ruolo del transfer e strategie di rifiuto in apprendenti ispanofoni di italiano L2. Uno studio esplorativo». Caruana et al. 2021, 115-26. http://doi.org/10.30687/978-88-6969-501-8/010.

De Mauro, T. (2018). L'educazione linguistica democratica. Roma-Bari: Laterza.

De Ponte, G. (2018). Global Compact on Migration e il Global Compact on Refugees: cosa porterà l'Europa alle negoziazioni? Gruppo migrazioni e Sviluppo. Concord Italia/Focsiv. https://tinyurl.com/yc6bsyw6.

De Spuches, G. (2014). «La città contemporanea di fronte al cultural turn». Governa, F.; Memoli, M. (a cura di), Geografie dell'urbano. Spazi, politiche pratiche della città. Roma: Carocci, 147-66.

Decimo, F. (2005). Quando emigrano le donne. Bologna: il Mulino.

Demetrio, D. (1997a). Agenda interculturale. Quotidianità e immigrazione a scuola. Idee per chi inizia. Milano: Meltemi. Contaminazioni 3.

Demetrio, D. (1997b). Manuale di educazione degli adulti. Roma-Bari: Laterza.

Diadori, P.; Palermo, M.; Troncarelli, D. (2021). Insegnare l'italiano come seconda lingua. Roma: Carocci.

Dryden-Peterson, S. (2015). The Educational Experiences of Refugee Children in countries of First Asylum. Washington DC: Migration Policy Institute.

Dürkheim, È. (1998). De la division du travail social. Paris: Presses Universitaires de France.

Edele, A. et al. (2015). «Why Bother with Testing? The Validity of Immigrants' Self-Assessed Language Proficiency». Social Science Research, 52, 99-123. https://doi.org/10.1016/j.ssresearch.2014.12.017.

Edelstein, C. (2000). «ll pozzo: uno spazio di incontri». Connessioni, 6, 71-86.

Edelstein, C. (2002). «Aspetti psicologici dei processi migratori al femminile - Albatros in volo». Psicologia e psicologi, 2(2), 227-43.

Edelstein, C. (2003). «Aspetti psicologici della migrazione al maschile e differenze di gender».M@gm@, Rivista Elettronica di Scienze Umane e Sociali - Osservatorio di Processi Comunicativi, 1(2). http://www. magma. analisiqualitativa.com/0102/article_06.htm.

Ekman, P. (1989). I volti della menzogna. Firenze: Giunti.

Ellis, N.C. (2005). «At the Interface: Dynamic Interactions of Explicit and Implicit Language Knowledge». Studies in Second Language Acquisition, 27(2), 30552. https://doi.org/10.1017/S○27226310505014X.

Erdocia, I. (2020). «The Politics of Plurilingualism: Immersion, Translanguaging, and School Autonomy in Catalonia». Linguistics and Education, 60, 1-12.

Ericson, R.V. (ed.) (1995). Crime and the Media. Dartmouth: Aldershot.

Ericson, R.V.; Baranek, P.M.; Chan, J.B.L. (1987). Visualising Deviance. Milton Keynes: Open University Press.

Erim, Y.; Morawa, E. (2016). «Psychotherapy with Immigrants and Traumatized Refugees». Psychother Psychosom Med Psychol, 66(9-10), 397-409. https:// doi.org/10.1055/s-0042-115412.

Eurydice (2005). L'educazione degli adulti in Europa. Firenze: Indire. Quaderni di Eurydice 25. 
Evans P. (1983). La motivazione. Bologna: Zanichelli.

Favaro, G. (1996). «Migrazione femminile e racconto di sé». Adultità, 4, 115-21.

Favaro, G. (2006). «Donne immigrate e formazione». Io parlo italiano. Corso di italiano L2. http://www.educational.rai.it/ioparloitaliano/ corso.htm.

Favaro, G.; Papa, N. (1997). La storia di Naima. Milano: In Dialogo.

Federighi, P. (2018). Educazione in età adulta. Ricerche, politiche, luoghie professioni. Firenze: Firenze University Press. https://doi.org/10.36253/97888-6453-752-8.

Ferrari, S. (2016). «Oggi facciamo pragmatica: un percorso di formazione e ricerca-azione nella scuola primaria». Italiano LinguaDue, 2, 270-80.

Ferreri, S. (2002). «Educazione linguistica: L1». Lavinio, C. (a cura di), La linguistica italiana alle soglie del 2000 (1987-1997 e oltre). Roma: Bulzoni, 213-52.

Fiorentino, G.; Cacchione, A. (2011). «Incremento lessicale e m(obile)-learning: prospettive teoriche e applicative». Italiano LinguaDue, 2, 201-17. https:// doi.org/10.13130/2037-3597/1920.

Floreancig, P. et al. (2018). Tecnologie, lingua, cittadinanza, percorsi di inclusione dei migranti nei CPIA. Milano: FrancoAngeli. http://library.oapen. org/handle/20.500.12657/27530

Fratzke, S.; Kainz, L. (2019). Preparing for the Unknown. Designing Effective Predeparture Orientation for Resettling Refugees. Brussels: Migration Policy Institute.

Freddi, G. (1987). «Dalla pedagogia alla glottodidattica». Titone, R. (a cura di), Functional Language Teaching Today / L'insegnamento funzionale delle lingue oggi. Milano: Oxford Institutes Italiani, 13-26.

Friedman, M.; Jaranson, J. (1994). «The Applicability of the Post-Traumatic Concept to Refugees». Marsella, A.J. et al. (eds), Amidst Peril and Pain. The Mental Health and Wellbeing of the World's Refugees. Washington DC: American Psychological Association, 207-27.

Gallina, V. (a cura di) (2006). Adult Literacy and Life Skills. Letteratismo e abilità per la vita. Popolazione 16-65 anni. Prima sintesi dei risultati. https://www. indire.it/lucabas/lkmw_file/eurydice/articolo_gallina.pdf.

García, O. (2017). «Problematizing Linguistic Integration of Migrants: The Role of Translanguaging and Language Teachers: Some Lessons from Research». Béacco, J.C. et al. (eds), The Linguistic Integration of Adult Migrants. Berlin, Boston: De Gruyter, 11-26.

Gavioli, L. (a cura di) (2009). La mediazione linguistico-culturale: un approccio interazionista. Perugia: Guerra.

Gavioli, L.; Baraldi, C. (2011). «Interpreter-Mediated Interaction in Healthcare and Legal Settings: Talk Organization, Context and the Achievement of Intercultural Communication». Interpreting 13(2), 205-33.

Gavioli, L.; Zorzi, D. (2008). «La partecipazione del paziente nell'interazione mediata con il medico: note linguistiche sulla dimensione informativa e sulla dimensione interpersonale». Baraldi, C. et al. (a cura di), Immigrazione, mediazione culturale e salute. Milano: Franco Angeli, 155-74.

Gianollo, C.; Fiorentini, I.; Grandi, N. (2020). La classe plurilingue. Bologna: Bononia University Press.

Gillespie, M. et al. (2016). Mapping Refugee Media Journeys: Smartphones and Social Media Networks. Walton Hall, Milton Keynes: The Open University / France Médias Monde. 
Ginnis, P. (2002). The Teacher's Toolkit: Raise Classroom Achievement with Strategies for Every Learner. New York: Crown House.

González, N. et al. (eds) (2005). Funds of Knowledge: Theorizing Practices in Households, Communities, and Classrooms. Mahwah (NJ): Lawrence Erlbaum.

González Garibay, M.; De Cuyper, P. (2013). The Evaluation of Integration Policies Across the OECD: A Review. Antwerpen: Steunpunt Inburgering en Integratie.

Grinberg, L.; Grinberg, R. (1990). Psicoanalisi dell'emigrazione e dell'esilio. Milano: FrancoAngeli.

Grosso, G.I. (2015). Interazioni in italiano lingua franca sul luogo di lavoro: una prospettiva pragmatica. Pisa: Edizioni ETS.

Grosso, G.I. (2021). «La formazione linguistica nei contesti delle attività prepartenza». Benucci et al. 2021, 27-52.

Grosso, G.I.; Floris, M. (2020). «L'italiano in ospedale. Analisi di interazioni e sperimentazione di un percorso didattico per bambini stranieri ospedalizzati». Marra, A.; Dal Negro, S. (a cura di), Lingue minoritarie tra localismi e globalizzazione. Milano: Officinaventuno, 101-18.

Grosso, G.I.; Pitzanti, G. (2019). «Dò il latte agli affamati. Collocazioni e frasi idiomatiche nell'italiano di stranieri: analisi di un corpus e proposte didattiche». Aldinucci, B. et al. (a cura di), Parola. Una nozione unica per una ricerca multidisciplinare. Siena: Edizioni Università per Stranieri di Siena, 551-62.

Grünhage-Monetti, M.; Braddell, A. (2017). «Integration... Needs Language, the Language of the Workplace: The Contribution of Work-related Second Language Learning to the Integration of Adult Migrants». Béacco, J.-C. et al., The Linguistic Integration of Adult Migrants, Some Lessons from Research. Strasbourg: CoE, 303-9.

Guardia, D. et al. (2016). «Prevalence of Psychiatric and Substance Use Disorders Among Three Generations of Migrants: Results from French Population Cohort». L'Encephale, 43(5), 435-43. http://doi.org/10.1016/j.encep.2016.06.008.

Guenier, N. (2002). "L'insécurité linguistique: objet divers, approches multiples». Bretegnier, A.; Ledegen, G. (éds), Sécurité/insécurité linguistique: terrains et approches diversifiés. Propositions théoriques et méthodologiques. Paris: L'Harmattan, 35-50.

Guidetti M.G.; Lenzi, G.; Storchi, S. (2012). «Potenzialità e limiti dell'uso dei corpora linguistici perla didattica dell'italiano LS». Bollettino Itals, aprile. https://tinyurl.com/bzkr33pk.

Haigler, K.O. et al. (1994). Literacy Behind Prison Walls. Washington DC: NAAL, NCES. Hall, E.T. (1959) The Silent Language. New York: Doubleday.

Hall, E.T. (1966) The Hidden Dimension. New York: Doubleday.

Hawley, J. et al. (2013). Prison Education and Training in Europe. Current State of Play and Challenges. Brussels: European Commission. http://www. antoniocasella.eu/nume/hawley_ue_education_may13.pdf.

Hidi, S. (1990). «An Interest Researcher's Prespective. The Effects of Exstrinsic and Intrinsic Factors on Motivation». Sansone, C.; Harackiewicz, J.M. (eds), Intrinsic and Exstrinsic Motivation. San Diego (CA): Academic Press, 309-39.

Hofstede, G. (2004). Cultures and Organizations: Software for the Mind. New York: McGraw Hill.

Hooft, H.; Schiepers, M.; Vandommele, G. (2021). «Developing and Validating a Multilingual Literacy Test for Asylum Seekers». Language Assessment Quarterly, 18, 1. https://doi.org/10.1080/15434303.2021.1931230. 
ICMC, International Catholic Migration Commission (2012). Welcome to Sheffield. Reflections on 8 Years Experience of Receiving Resettled Refugees at the Local Level. Brussels: ICMC Europe. https://www. resettlement.eu/ sites/icmc.tttp.eu/files/ICMC_WelcomeToSheffield.pdf.

ICMC (2015). Volunteering for Refugee Integration: A SHARE Network Toolkit Publication. Brussels: ICMC Europe. https://resettlement.eu/page/volunteering-refugee-integration-share-network-toolkit-publication.

ICMC; Vluchtelingen Werk Limburg (2014). 'What makes volunteering work?' Supporting reception, building integration \& promoting independence through volunteering. Maastricht. http://www. resettlement.eu/sites/icmc. tttp.eu/files/FINAL_SHARE_Volunteer\%20report_๑.pdf.

IOM, International Organization of Migration (2011). IOM Project Handbook. Geneva. https://publications.iom.int/system/files/pdf/iom_project_handbook_6feb2012.pdf.

IOM (2016). Study on Migrants' Profiles, Drivers of Migration and Migratory Trends. Rome: IOM. https://italy.iom.int/en/activities/research/study-migrants'-profiles-drivers-migration-and-migratory-trends.

IOM (2018). EU Integration Practices Brochure. EU Exchange on Integration Practices for Resettled Refugees (Bucharest, 20 April 2018). https://www.ritaresources.org/wp-content/uploads/2019/01/LINK-IT-EU-Integration-Practices-Brochure-Final-1.pdf.

IOM (2019a). IOM Resettlement. The UN Migration Agency. Geneva. https:// publications.iom.int/system/files/pdf/rst_booklet_2019.pdf.

IOM (2019b). Reintegration Handbook. Practical Guidance on the Design, Implementation and Monitoring of Reintegration Assistance. Geneva. https://publications.iom.int/es/books/reintegration-handbookpractical-guidance-design-implementation-and-monitoringreintegration.

IOM (2019c). Resettlement Enhanced International Commitment to Safe Migration. Geneva. https://publications.iom.int/system/files/pdf/resettlement-booklet-2020.pdf.

IOM (2020a). Migration, Environment and Climate Change: Literature Review. First Report. Global Migration Data Analysis Centre (GMDAC). Berlin: IOM.

IOM (2020b). World Migration Report. Geneva: IOM.

IRCC (2018). Evaluation of Pre-Arrival Settlement Services. Research and Evaluation Branch, Canada. https://www.canada.ca/content/dam/ircc/ documents/pdf/english/evaluation/e3-2017-pre-arrival-en.pdf. Irigaray, L. (1992). Je, tu, nous. Paris: Grasset et Fasquelle.

ISFOL (2014). PIAAC-OCSE. Rapporto nazionale sulle Competenze degli Adulti. https://www.isfol.it/piaac/Rapporto_Nazionale_Piaac_2014.pdf.

Kasper, G.; Blum-Kulka, S. (eds) (1993). Interlanguage Pragmatics. New York: Oxford University Press.

Kasper, G.; Rose, K. (2003). Pragmatic Development in a Second Language. New York: Blackwell.

Kendrick, M.; Kakuru, D. (2012). «Funds of Knowledge in Child-headed Households: A Ugandan Case Study». Childhood, 19(3), 397-413. https://doi. org/10.1177/0907568212439587.

Klein, E.; Martohardjono, G. (2015). «English Language Learners with Low Native Language Literacy: A Profile and Intervention in New York City». Mari- 
cel, S.; Whiteside, A. (eds), Proceedings of the Ninth LESLLA Symposium, 2013. San Francisco: Lulu Publishing Services, 174-95.

Knowles, M.S. (1993). Quando l'adulto impara: pedagogia e andragogia. Milano: FrancoAngeli.

Kramsch, C. (1993). Context and Culture in Language Teaching. Oxford: Oxford University Press.

Krashen, S.D.; Long, M.H.; Scarcella, R.C. (1982). Child-Adult Differences in Second Language Acquisition. New York: Newbury House Publishers.

Kukulska-Hulme, A. et al. (2017). «Mobile Language Learning Experiences for Migrants Beyond the Classroom». Béacco, J.-C. et al. (eds), The Linguistic Integration of Adult Migrants / L'intégration linguistique des migrants adultes. Some Lessons from Research / Les enseignements de la recherche. Berlin: De Gruyter Mouton, 219-24.

Kukulska-Hulme, A.; Lee, H.; Norris, L. (2017). «Mobile Learning Revolution: Implications for Language Pedagogy». Chapelle, C.A.; Sauro, S. (eds), The Handbook of Technology and Second Language Teaching and Learning. Oxford: Wiley \& Sons, 217-33.

La Grassa, M.; Troncarelli, D. (2015). «L'italiano online: pubblici, percorsi e prospettive». Ramsey-Portolano, C. (ed.), The Future of Italian Teaching: Media, New Technologies and Multi-Disciplinary Perspectives. Newcastle upon Tyne: Cambridge Scholars, 2-21.

Lafon, J.-C. (1969). «Audition et langage». Le langage: approches scientifiques et philosophiques. Paris: Lethiellcux, 31-74.

Lastrina, O. (2017). «Interazione geni-ambiente ed esordio psicotico, un focus sull'esperienza migratoria». State of Mind, 29 marzo. https://www.stateofmind.it/2017/03/migrazione-psicosi/.

Leone, P. (2011). «ll litigio scortese: pratiche comunicative e didattica del parlato». Leone, P.; Mezzi, T. (a cura di), Didattica della comunicazione orale. Milano: FrancoAngeli, 96-113.

Li, S.S.Y.; Liddell, B.J.; Nickerson, A. (2016). «The Relationship Between Post-Migration Stress and Psychological Disorders in Refugees and Asylum Seekers». Curr Psychiatry Rep, 18(9), 82. https://doi.org/10.1007/s11920๑16-๑723-๑.

Liddicoat, A.J. (2016). «Native and Non-Native Speaker Identities in Interaction: Trajectories of Power». Applied Linguistics Review, 7(4), 409-29. http:// dx.doi.org/10.1515/applirev-2016-๑๑18.

Lier, L. von (2004). Ecology and Semiotic of Language Learning. Boston: Kluwer.

Ligas, G. (2021). L'Educazione Linguistica Democratica per il contrasto del fallimento formativo e per l'inclusione sociale: dagli aspetti storico-teorici alla percezione della 'democraticità' nei processi di apprendimento/insegnamento [Tesi di Laurea Magistrale]. Cagliari: Università degli Studi di Cagliari.

Lolk, M. et al. (2012). «Somatic Comorbidity Among Migrants with Posttraumatic Stress Disorder and Depression: A Prospective Cohort Study». BMC Psychiatry, 16, 447. https://doi.org/10.1186/s12888-016-1149-2.

Majhanovich, S.; Deyrich, M.-C. (2017). «Language Learning to Support Active Social Inclusion: Issues and Challenges for Lifelong Learning». International Review of Education, 63. https://doi.org/10.1007/s11159017-9656-z.

Malandrino, A. (2021). «A Research Agenda for a Multilingual Education Policy in Immigratory Contexts». Scienza e Pace, 12(1), 69-87. 
Malinowski, D.; Maxim, H.H.; Dubreil, S. (eds) (2021). Language Teaching in the Linguistic Landscape: Mobilizing Pedagogy in Public Space. Cham: Springer Nature.

Månsson, J.; Delander, L. (2017). «Mentoring as a Way of Integrating Refugees into the Labour Market: Evidence from a Swedish Pilot Scheme». Economic Analysis and Policy, 56, 51-9.

Marelli, M.; Rodondi, F. (2013). Parole in movimento. Percorso di alfabetizzazione e educazione alla cittadinanza. Bergamo: Sestante.

Marini, A. (2001). Elementi di psicolinguistica generale. Berlino: Springer.

Mayr, G. (2021). «Paesaggi linguistici e scrittura plurilingue. Un'analisi di testi plurilingui di allievi/e delle scuole secondarie di secondo grado». Caruana et al. 2021, 189-98. http://doi.org/10.30687/978-88-6969-501-8/017.

McClelleland, D.C.; Koestner, R.; Weinberger, J. (1989). «How Do Self-Attributed and Implicit Motives Differ?». Psychological Review, 96(4), 690-702. https://doi.org/10.1037/0033-295X.96.4.690.

Mehrabian, A. (1972). Nonverbal Communication. Berlin: De Gruyter.

Meißner, F.-J. et al. (2011). Intercomprehension: Learning, Teaching, Research. Apprentissage, enseignement, recherche. Lernen, Lehren, Forschung. Tübingen: Narr.

Minascurta, M. (2017). «L'importanza dei valori culturali nella comunicazione interculturale: italiani e romeni a contatto». EL.LE, 6(1), 85-108. http:// doi.org/10.14277/2280-6792/ELLE-6-1-17-5.

Mineo, S.; Amendola, M. (a cura di), (2018). Focus PIAAC: i low skilled in literacy. Profilo degli adulti italiani a rischio di esclusione sociale. Roma: INAPP.

Minuz, F. (2005). Italiano L2 e alfabetizzazione in età adulta. Roma: Carocci.

Minuz, F. (2019). «L'apprendente adulto analfabeta: una definizione internazionale». Caon, F.; Brichese, A. (a cura di), Insegnare italiano ad analfabeti. Torino: Bonacci-Loescher, 19-20.

Minuz, F.; Borri, A. (2016). "Literacy and Language: Tools, Implementation and Impact». Italiano LinguaDue, 8, 220-31. https://doi. org/10.13130/2037-3597/8184.

Minuz, F.; Rocca, L.; Borri, A. (2014). Italiano L2 in contesti migratori. Sillabo e descrittori dall'alfabetizzazione all'A1. Torino: Loescher. I quaderni della ricerca 17.

Minuz, F.; Rocca, L.; Borri, A. (2017). «Syllabus and Descriptors for Illitterate, Semi-Literate and Literate Users. From Illiteracy to A1 Level». Sosiński, M. (ed.), Alfabetización y aprendizaje de idiomas para adultos. Investigación, política educativa y práctica docente. Literacy Education and Second Language Learning by Adults, Research, Policy and Practice. Granada: Editorial Universidad de Granada, 207-18.

Moè, A. (2010). La motivazione. Bologna: il Mulino.

Monaci, V. (2021). «Strumenti di valutazione per attività pre-partenza». Benucci et al. 2021, 115-43.

Montero, M.K. (2018). «Narratives of Trauma and Self-healing Process in a Literacy Program for Adolescent Refugee Newcomers». Shapiro et al. 2018, 92-106.

Morgan, M.; Kett, M. (2003). The Prison Adult Literacy Survey. Results and implications. Dublin: Irish Prison Service.

Murillo, J.P.; Harmegnies, B. (2005). «Fattori e strategie d'intercomprensione spontanea per la sopravvivenza comunicativa nel mondo romanzo». Benucci, A. (a cura di), Le lingue romanze. Una guida per l'intercomprensione. Torino: UTET Libreria, 89-115. 
Narcy-Combes, J.-P.; Narcy-Combes, M.-F. (2019). Cognition et personnalité dans l'apprentissage des langues. Relier théories et pratiques. Paris: Didier.

Nuzzo, E. (2016). «Fonti di input per l'insegnamento della pragmatica in italiano L2: riflessioni a partire dal confronto tra manuali didattici, serie televisive e parlato spontaneo». Santoro, E.; Vedder, I. (a cura di), Pragmatica e interculturalità in italiano lingua seconda. Firenze: Franco Cesati, 15-27.

Nuzzo, E.; Gauci, P. (2012). Insegnare la pragmatica in italiano L2. Recenti ricerche nella prospettiva della teoria degli atti linguistici. Roma: Carocci.

Nuzzo, E.; Zanoni, G. (2012). «Il progetto LIRA: un repository multimediale per lo sviluppo delle competenze pragmatiche in parlanti non nativi d'italiano». Mello, H. et al. (eds), Proceedings of the VIIth GSCP International Conference. Speech and Corpora. Firenze: Firenze University Press. http://150.164.100.248/gscp2012-eng/data1/arquivos/86.pdf.

O'Reilly, K. (2012). International Migration and Social Theory. Basingstoke: Palgrave Macmillan.

Ochs, E. (2012). «Experiencing Language». Sage Journals, 12(2), 142-60.

OCDE (2002). Glossary of Key Terms in Evaluation and Results-Based Management. Paris. https://www.oecd.org/dac/evaluation/2754804.pdf.

OECD (2019a). «Performance in Reading Components Across Countries: Average Proportion of Items Answered Correctly, Adults Who Score at or Below Level 1 in Literacy Proficiency». Skills Matter:Additional Results from the Survey of Adult Skills, OECD Skills Studies. Paris: OECD Publishing.

OECD (2019b). Better Criteria for Better Evaluation. Revised Evaluation Criteria. Definitions and Principles for Use. https://www.oecd.org/dac/evaluation/revised-evaluation-criteria-dec-2019.pdf.

Orletti, F. (2000). La conversazione diseguale. Potere e interazione. Roma: Carocci.

Orletti, F.; Fatigante, M. (a cura di) (2013). «La sfida della multiculturalità nell'interazione medico-paziente». Salute e società, 12(1), 15-17.

Osgood, C.E.; Sebeok, T.A. (eds) (1965). Psycholinguistics. Bloomington (IN): Indiana University Press.

Palermo, M. (2013). Linguistica testuale dell'italiano. Bologna: il Mulino.

Pallotti, G. (2007). «Conversation Analysis: Methodology, Machinery and Application to Specific Settings». Bowles, H.; Seedhouse, P. (eds), Conversation Analysis and Language for Specific Purposes. Bern: Peter Lang, 37-67.

Park, K.; Valdez, V.E. (2018). «Translaguaging Pedagogy to Support the Language Learning of Older Nepali Bhutanese Adults». Shapiro et al. 2018, 49-65.

Paulsrud, B.A. et al. (2020). «Spaces for Multilingual Education: Language Orientations in the National Curricula of Sweden and Finland». International Multilingual Research Journal, 14(4), 304-18. https://doi.org/10.1080/ 19313152.2020.1714158.

Pavlenko, A. (2007). «Autobiographic Narratives as Data in Applied Linguistics». Applied Linguistics, 28(2), 163-88. http://doi.org/10.1093/app-

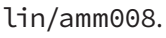

Pease, A. (1998). Leggere il linguaggio del corpo. Milano: Mondadori.

Pellitteri, A. (2015). «Alfabetizzazione, un vecchio problema e una nuova sfida». Amoruso, M.; D’Agostino, M.; Jaralla Y.L. (a cura di), Dai barconi all'università-Percorsi di inclusione linguistica per minori stranieri non accompagnati. Palermo: Scuola di Lingua italiana per Stranieri, Università di Palermo. Disponibile alla pagina: https://minorinonaccompagnatialluniversita.wordpress.com/dentro-la-classe/. 
Pennycook, A. (2012). Language and Mobility. Unexpected Places. Salisbury: Short Run Press.

Peppoloni, D. (2018). Glottodidattica e metalinguaggio. La consapevolezza metalinguistica come strumento per l'acquisizione delle lingue straniere. Perugia: Guerra.

Pintrich, P.R. (2003). «A Motivational Science Perspective on the Role of Student Motivation in Learning and Teaching Contexts». Journal of Educational Psychology, 95(4), 667-86.

Priebe, S.; Giacco, D.; El-Nagib, R. (2016). Public Health Aspects of Mental Health Among Migrants and Refugees: A Review of the Evidence on Mental Health Care for Refugees, Asylum Seekers and Irregular Migrants in the WHO European Region. Health Evidence Network synthesis report 47.

QCER (2002). Quadro Comune Europeo di riferimento per le lingue: apprendimento insegnamento valutazione. Milano: La Nuova Italia-Oxford.

QCER (2020). Quadro comune europeo di riferimento per le lingue: apprendimento, insegnamento, valutazione. Milano: La Nuova Italia.

Quercioli, F. (2004). «L'insegnamento dell'italiano lingua seconda a donne immigrate. Caratteristiche specifiche e scelte didattiche». Bollettino per la didattica nella classe plurilingue, 9. http://www. dimasystems.it/gioco-degli-specchi/wp-content/uploads/2014/01/Corsi-per-donne.pdf.

Rapporto Annuale SPRAR (2016). Sistema di protezione per richiedenti asilo e rifugiati. Atlante SPRAR. Roma: Ministero dell'Interno.

Rapporto Annuale SPRAR/SIPROIMI (2018). Rapporto annuale Sprar/Siproimi. Sistema di protezione per titolari di protezione internazionale e per minori stranieri non accompagnati. Roma: Ministero dell'Interno.

Rastelli, S. (2009). Che cos'è la didattica acquisizionale. Roma: Carocci.

Read, T. (2020). «Towards a New Model for Inclusive Education Based on Virtual Social Inclusion and Mobile Openness». World Journal on Educational Technology: Current Issues, 12(1), 14-22.

Rechel, B. et al. (2011). Migration and Health in the European Union: an introduction. Maidenhead: Open University Press.

Ripley Crandall, B. (2018). «'History Should Come first': perspectives of Somali born Refugee Background Male Youth on Writing in and out of School». Shapiro et al. 2018, 33-48.

Rocca, L. (2019). «La gestione della fase di accoglienza dell'utenza vulnerabile: intervista e test». Caon, F.; Brichese, A. (a cura di), Insegnare italiano ad analfabeti. Torino: Bonacci-Loescher, 69-88.

Rommetveit, R. (1974). On Message Structure. New York: Academic Press.

Sabatini, F. (1990). La comunicazione e gli usi della lingua. Torino: Loescher.

Saggiomo, V. (2019). «Evaluation: An Opportunity for Improvement. In International Organization for Migration». Saggiomo, V. (a cura di), Project Development Manual for Migrants' Associations. Global Development Agents. Rome: IOM. https://publications.iom.int/system/files/pdf/project_dev_manual_en.pdf.

Şalli-Çopur, D. (2005). «Coping with the Problems of Mixed Ability Classes». The Internet TESL Journal, 11(8). http://iteslj.org/Techniques/Salli-Copur-MixedAbility.html.

Santerini, M. (2015). Vivere nel pluralismo. L'educazione alla cittadinanza in prospettiva interculturale. https://tinyurl.com/5dwaj2b2.

Santipolo, M. (2018a). «Continuità nel cambiamento: RILA, un macrotesto linguistico tra sincronia e diacronia». RILA, 1, 7-13. 
Santipolo, M. (2018b). «Consapevolezza ed educazione linguistica». RILA, 2/3, 7-19.

Santoro, E.; Vedder, I. (a cura di) (2016). Pragmatica e interculturalità in italiano lingua seconda. Firenze: Cesati.

Savoia, T. (2013). Crimine, detenzione, educazione. Studio sui detenuti della casa di reclusione di Spoleto [tesi di dottorato]. Roma: Università degli Studi di Roma Tre.

Scheible, J.A. (2018). Literacy Training and German Language Acquisition Among Refugees: Knowledge of German and the Need for Support Among Integration Course Attendees Learning a Second Alphabet and Those with No Literacy Skills (BAMF-Brief Analysis, 1-2018). Nürnberg: Bundesamt für Migration und Flüchtlinge (BAMF) Forschungszentrum Migration, Integration und Asyl (FZ). https://nbn-resolving.org/urn:nbn:de:0168ssoar-67560-1.

Schmidt, R.W. (1990). «The Role of Consciousness in Second Language Learning». Applied Linguistics, 11, 129-58.

Schmidt, T. (2017). «Inklusiven Fremdspracheunterricht gestalten - Von Theorie Praxis-Netzwerken, multiprofessionellen Teams und interdisziplinärer Forschung». Burwitz-Melzer, E. et al. (Hrsgg), Inklusion, Diversität und das Lehren und Lernen fremder Sprachen. Arbeitspapiere der 37. Frühjahrskonferenz zur Erforschung des Fremdsprachenunterrichts. Giessener Beiträge zur Fremdsprachendidaktik. Tübingen: Narr, 285-95.

Sciuti Russi, G.; Carmignani, S. (a cura di) (2015). La formazione in contesto penitenziario. Percorsi di lingua e insegnamento a detenuti stranieri adulti. Pisa: Pacini.

Shapiro, S. et al. (eds) (2018). Educating Refugee-background Students. Bristol: Multilingual Matters.

Shohamy, E. et al. (eds) (2010). Linguistic Landscape in the City. Bristol: Multilingual Matters.

Siebetcheu, R. (2011). «Identità e ruolo del mediatore linguistico-culturale in Italia». The Journal of Cultural Mediation, 1, 7-16.

Siebetcheu, R. (2020). «Strategie didattiche nelle classi plurilingui: una sperimentazione in contesto sportivo». Italiano LinguaDue, 2, 390-403. https:// doi.org/10.13130/2037-3597/15086.

Simonin, J. (1996). «A la Réunion, peut-on être sûr d'être insécure? Pour un traitement interactionnel de l'insécurité linguistique». Bavoux, C. (éd.), Français régionaux et insécurité linguistiques. Paris: L'Harmattan, 33-54.

Skinner, B.F. (1974). About Behaviourism. New York: Knopf.

Sluzki C. (1979). «Migration and Family Conflict». Family Process, 18(4), 379-90.

Smythe, F. (2020). «Language Inclusiveness in Edcation: Implications for Immigrant Students in France and New Zealand». New Zealand Journal of Educational Studies, 55, 215-46.

Solcia, V. (2011). «Non solo lingua. I corsi di italiano L2 per donne migranti tra bisogni linguistici e desiderio di integrazione». Italiano LinguaDue, 2, 129200. https://doi.org/10.13130/2037-3597/1919.

Spano, C. (2020). Analisi degli studi sull'alfabetizzazione degli adulti: dalle ricerche internazionali all'attività nei centri provinciali per l'istruzione degli adulti [tesi di laurea magistrale]. Cagliari: Università degli Studi di Cagliari.

Spinelli, B.; Parizzi, F. (2010). Profilo della lingua italiana. Firenze: La Nuova Italia. 
Spitz, G.; Muskens, R.; van Ewijk, E. (2013). The Dutch and Development Cooperation. Amsterdam. http://www.ncdo.nl/sites/default/files/Report\%20Analysis\%20The\%20Dutch\%20and\%20Development\%20Cooperation\%20FINAL\%202013\%2003\%20๑4.pdf.

SPRAR (2010). Le dimensioni del disagio mentale nei richiedenti asilo e rifugiati. Problemi aperti e strategie di intervento. Roma: CITTALIA. https:// tinyurl.com/4m7fmdu5.

Steel, P. et al. (2017). «The Happy Culture: A Theoretical, Meta-Analytic, and Empirical Review of the Relationship Between Culture and Wealth and Subjective Well-Being». Personality and Social Psychology Review, 22(2), 128-69. https://doi.org/10.1177/1088868317721372.

Steinberg, D.D.; N.V. Sciarini (2006). «An Introduction to Psycholinguistics». Neuphilologische Mitteilungen, CIX(4), 492-5.

Tarone, E. et al. (2009). Literacy and Second Language Oracy. Oxford: Oxford University Press.

Tarone, E.; Bigelow, M. (2005). «Impact of Literacy on Oral Language Processing: Implications for Second Language Acquisition Research». Annual Review of Applied linguistics, 25, 77-97. https://doi.org/10.1017/

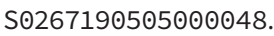

Taronna, A. (2015). "La mediazione linguistica come pratica di negoziazione, resistenza, attivismo e ospitalità sulle sponde del Mediterraneo». Lingue Linguaggi 16, 159-75.

Tice, J. (1997). The Mixed Ability Class. London: Richmond.

Titone, R. (1971). Psicolinguistica applicata. Roma: Armando.

Tognetti Bordogna, M. (2012). Donne e percorsi migratori. Per una sociologia delle migrazioni. Milano: FrancoAngeli.

Translators Without Borders (2017). Research Report. Putting Language on the Map in the European Refugee Response. https://translatorswithoutborders.org/wp-content/uploads/2017/04/Putting-languageon-the-map.pdf.

Trotta, G. (2021). «Modelli di competenza comunicativa interculturale». Benucci et al. 2021, 53-82.

Trubnykova, V. (2021). "Scusi, guardi che stavo aspettando io per il parcheggio'. Protestare in una prospettiva cross-culturale». Caruana et al. 2021, 73-84. http://doi.org/10.30687/978-88-6969-501-8/०06.

UNDP (2009). Handbook on Planning, Monitoring and Evaluating for Development Results. http://web.undp.org/evaluation/handbook/documents/english/pme-handbook.pdf.

UNEG (2016). Norms and Standards for Evaluation. http://www. unevaluation.org/document/detail/1914.

UNESCO (2006). Education for All Global Monitoring Report. Paris: UNESCO.

UNHCR (2002). Refugee Resettlement. An International Handbook to Guide Reception and Integration. https://www.unhcr.org/protection/ resettlement/4a2cfe336/refugee-resettlement-international-handbook-guide-reception-integration.html.

UNHCR (2019). Global Resettlement Needs. https://www.unhcr.org/protection/resettlement/5b28a7df4/projected-global-resettlement-needs-2019.html.

Ur, P. (1996). A Course in Language Teaching: Theory and Practice. Cambridge: Cambridge University Press. 
Urdan, T.C.; Maehr, M.L. (1995) «Beyond a Two-Goal Theory of Motivation and Achievement: a Case for Social Goals». Review of Educational Research, 65, 213-43.

Van Berne, T. (2016). Perché non sei mai puntuale? Analisi comparativa della concezione del tempo nella cultura italiana e quella olandese [tesi di laurea magistrale]. Utrecht: Utrecht University.

Van de Craats, I.; Kurvers, J.; Young-Scholten, M. (2006). «Research on Loweducated Second Language and Literacy Acquisition». LOT Occasional Series, 6, 7-23.

Van der Kolk, B.A. (2004). «Psychobiology of Posttraumatic Stress Disorder». J Clin Psychiatry, 58(9), 16-24. https://pubmed.ncbi.nlm.nih. gov/9329447/.

Vedovelli, M. (2002a). Guida all'italiano per stranieri. La prospettiva del Quadro comune europeo per le lingue. Roma: Carocci.

Vedovelli, M. (2002b). L'italiano degli stranieri. Storia, attualità e prospettive. Roma: Carocci.

Vedovelli, M. (2010). Guida all'italiano per stranieri. Dal Quadro Comune Europeo per le lingue alla Sfida salutare. Roma: Carocci.

Vedovelli, M. (2012). «Mutamenti sociali e scenari linguistici per l'immigrazione straniera in Italia al tempo della crisi». Bollettino di italianistica, 2, 48-65.

Vedovelli, M. (a cura di) (2013). «La migrazione globale delle lingue. Lingue in (super-) contatto nei contesti migratori del mondo globale». Centro Studi Emigrazione, 191, 419-506.

Vedovelli, M.; Villarini, A. (2003). «Dalla linguistica acquisizionale alla didattica acquisizionale: le sequenze sintattiche nei materiali per l'italiano $L 2$ destinati agli immigrati stranieri». Giacalone Ramat, A. (a cura di), Verso l'italiano. Percorsi e strategie di acquisizione. Roma: Carocci, 270-304.

Veneri, A. (2004). L'italiano con Naima. Milano: Guerini.

Vinogradov, P.; Bigelow, M. (2010). «Using Oral Language Skills to Build on the Emerging Literacy of Adult English Learners». CAELA Network Brief, 2-8. https://www.cal.org/adultesl/pdfs/briefs/using-oral-language-skills.pdf.

Virgilio, F. (2018). «Presentazione». Floreancig, P. et al., Tecnologie, lingua, cittadinanza, percorsi di inclusione dei migranti nei CPIA. Milano: FrancoAngeli, 7-14. http://library.oapen.org/handle/20.500.12657/27530.

Wadsworth, T. (2010). «Is Immigration Responsible for the Crime Drop? An Assessment of the Influence of Immigration on Changes in Violent Crime Between 1990 and 2000». Social Science Quarterly, 91, 531-53.

Webb, S. et al. (2016). «Refugee Migration, Lifelong Education and Forms of Integration». International Journal of Lifelong Education, 35(3), 213-15.

Weinreich, U. (1974). Lingue in contatto. Torino: Boringhieri.

Whittal, A.; Lippke, S. (2015). «Investigating Patients with an Immigration Background in Canada: Relationships Between Individual Immigrant Attitudes, the Doctor-Patient Relationship, and Health Outcomes». BMC Public Health, 16(23). https://bmcpublichealth.biomed.

Wihtol de Wenden, C. (2017). «Actual Patterns of Migration Flows. The Challenge of Migration and Asylum in Contemporary Europe». Grimmel, A.; My Giang, S. (eds), Solidarity in the European Union. Berlin: Springer, 67-80.

Wrigley, H.S.; Guth, G.J.A. (1992). Bringing Literacy to Life Issues and Options in Adult ESL Literacy. San Mateo (CA): Aguirre International. 
Wrigley, H.S.; Guth, G.J.A. (2000). Bringing Literacy to Life: Issues and Options in Adult ESL Literacy. San Mateo (CA): Aguirre International.

Zanoni, G. (2017). «L'interazione tra parlanti di italiano L1 e L2 nel forum linguistico di LIRA». Andorno, C.; Grassi, R. (a cura di), Le dinamiche dell'interazione. Milano: Officinaventuno, 203-16.

Zoletto, D. (2018). «Eterogeneità, inclusione, cittadinanza. Prospettive e sfide pedagogiche nel contesto dei Centri Provinciali di Istruzione per gli Adulti». Floreancig, P. et al., Tecnologie, lingua, cittadinanza, percorsi di inclusione dei migranti nei CPIA. Milano: FrancoAngeli, 32-41. http://library.oa-

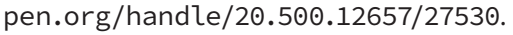

\section{Documenti di politica linguistica e riferimenti legislativi}

Aebi, M.F.; Hashimoto, Y.Z. (2018). SPACE II-2018-Council of Europe Annual Penal Statistics: Persons under the supervision of Probation Agencies. Strasbourg: Council of Europe.

Agenda europea rinnovata per l'apprendimento degli adulti (2011/C 372/01). Risoluzione del Consiglio. Gazzetta ufficiale dell'Unione Europea C 372/1. 20.12.2011.

AUSCO, Australian Cultural Orientation (s.d.). https://www.iom.int/australian-cultural-orientation, https://ec.europa.eu/migrantintegration/integration-practice/mentoring-program-migrants_en.

Commission of the European Communities (2005). A European Information Society for Growth and Employment. http://eurlex.europa.eu/LexUriServ/LexUriServ.do?uri=COM:2005:๑229:FIN:EN:PDF.

Commissione delle Comunità Europee (2000). Documento di lavoro dei servizi della Commissione. Memorandum sull'Istruzione e l'Educazione permanente. SEC(2000) 1832. https://archivio.pubblica.istruzione.it/dg_ postsecondaria/memorandum.pdf.

Commissione delle Comunità Europee (2006). Comunicazione della Commissione. Educazione degli adulti: non è mai troppo tardi per apprendere. $\operatorname{COM(2006)614.~https://eur-lex.europa.eu/legal-content/IT/~}$ TXT/PDF/?uri=CELEX:52006DC0614\&from=EN.

Commissione Europea (2020). Piano d'azione per l'integrazione e l'inclusione 2021-2027. https://www.lavoro.gov.it/temi-e-priorita/immigrazione/Documents/Piano-azione-per-integrazione-e-inclusione-2021-2027.PDF.

Commissione Europea/EACEA/Eurydice (2015). Istruzione e formazione degli adulti in Europa: ampliare l'accesso alle opportunità di apprendimento. Rapporto Eurydice. Lussemburgo: Ufficio delle pubblicazioni dell'Unione europea. https://www.indire.it/lucabas/Lkmw_img/eurydice/Adult_ Education_Training_IT.pdf.

Comunicazione della Commissione Europa (2020). Una strategia per una crescita intelligente, sostenibile e inclusiva. COM(2010) 2020.

Consiglio europeo di Lisbona (2000). European Parliament. https://www.europarl.europa.eu/summits/lis1_it.htm.

Direttiva 2000/43/CE del Consiglio del 29 giugno 2020 che attua il principio della parità di trattamento fra le persone indipendentemente dalla raz- 
za e dall'origine etnica. Gazzetta ufficiale delle Comunità europee, L 180, 19/07/2000, 22-6.

Direttiva 2000/78/CE del Consiglio del 27 novembre 2000 che stabilisce un quadro generale per la parità di trattamento in materia di occupazione e di condizioni di lavoro. Gazzetta ufficiale delle Comunità europee, L 303, 02/12/2000, 16-22.

DPR nr. 263, del 209 ottobre 2012. Regolamento recante norme generali per la ridefinizione dell'assetto organizzativo didattico dei Centri d'Istruzione per gli Adulti, ivi compresi i corsi serali, a norma dell'articolo 64, comma 4, del decreto-legge 25 giugno 2008, nr. 112, convertito, con modificazioni, dalla legge 6 agosto 2008, nr. 133. Gazzetta Ufficiale della Repubblica Italiana, Serie Generale nr. 47 del 25/02/2013.

OECD; European Union (2018). Settling In 2018: Indicators of Immigrant Integration. Paris; European Union; Brussels: OECD Publishing. https://doi. org/10.1787/9789264307216-en.

Rapporto KOK (2004). Affrontare la sfida. Strategia di Lisbona per la crescita e l'occupazione. Relazione del gruppo ad alto livello presieduto da Wim Kok, Novembre.

Relazione (2021). Relazione della Commissione al Parlamento Europeo e al Consiglio sull'applicazione della direttiva 2000/43/CE del Consiglio che attua il principio della parità di trattamento fra le persone indipendentemente dalla razza e dall'origine etnica («direttiva sull'uguaglianza razziale»). Bruxelles: Commissione Europea.

Trattato sull'Unione Europea. Gazzetta ufficiale dell'Unione Europea, C 326, 26/10/2012, 13-45.

White Paper on Intercultural Dialogue (2008). «Living Together As Equals in Dignity». Strasbourg: Council of Europe Ministers of Foreign Affairs.

UNESCO (2005). Aspects of Literacy Assessment: Topics and issues from the UNESCO Expert Meeting (UNESCO Paris, 10-12 June 2003). https://unesdoc. unesco.org/ark:/48223/pfø0๑९140125. 


\section{SAIL}

1. Caon, Fabio (2012). Aimes-tu le français? Percezione dello studio obbligatorio del francese nella scuola media.

2. Serragiotto, Graziano (2012). Cosa ti hanno insegnato al liceo? La percezione di studenti di Lingue sulla loro formazione linguistica.

3. Balboni, Paolo; Daloiso, Michele (2012). La formazione linguistica nell'università.

4. Novello, Alberta (2014). La valutazione delle lingue straniere e seconde nella scuola. Dalla teoria alla pratica.

5. Freddi, Edigio (2015). Acquisizione della Lingua Italiana e adozione internazionale. Una prospettiva linguisitica.

6. Carloni, Giovanna (2015). CLIL in Higher Education and the Role of Corpora. A Blended Model of Consultation Services and Learning Environments.

7. Melero Rodríguez, Carlos Melero (a cura di) (2016). Le lingue in Italia, le lingue in Europa: dove siamo, dove andiamo.

8. Cavaliere, Salvatore (2016). Tra lingue e culture. La comunicazione interculturale tra italiani e popoli slavi meridionali.

9. Bonvino, Elisabetta; Jamet, Marie-Christine (a cura di) (2016). Intercomprensione: lingue, processi e percorsi.

10. Dalla Libera, Cristina (2017). Tra lingue e culture. La comunicazione interculturale fra italiani e russi.

11. Maugeri, Giuseppe (2017). La progettazione degli ambienti didattici per l'apprendimento delle lingue straniere.

12. Newbold, David (2017). Rethinking English Language Certification. New Approaches to the Assessment of English as an Academic Lingua Franca.

13. Bier, Ada (2018). La motivazione nell'insegnamento in CLIL.

14. Balboni, Paolo (2018). Sillabo di riferimento per l'insegnamento dellitaliano della musica.

15. Spaliviero, Camilla (2020). Educazione letteraria e didattica della letteratura.

16. Castagna, Vanessa; Quarezemin, Sandra (org.) (2020). Travessias em língua portuguesa. Pesquisa linguística, ensino e tradução. 
17. Daloiso, Michele; Mezzadri, Marco (a cura di) (2021). Educazione linguistica inclusiva. Riflessioni, ricerche ed esperienze.

18. Caruana, Sandro; Chircop, Karl; Gauci, Phyllisienne; Pace, Mario (a cura di) (2021). Politiche e pratiche per l'educazione linguistica, il multilinguismo e la comunicazione interculturale.

19. Maugeri, Giuseppe (2021). L'insegnamento dell'italiano a stranieri. Alcune coordinate di riferimento per gli anni Venti.

20. Maugeri, Giuseppe; Serragiotto, Graziano (2021). L'insegnamento della lingua italiana in Giappone. Uno studio di casa sul Kansai. 

Il presente volume cerca di offrire risposte alle molteplici sfide che caratterizzano tutti coloro che, a diverso titolo, partecipano all'Educazione Linguistica in contesti connotati dalla presenza di persone migranti. II problema viene affrontato da una prospettiva interdisciplinare e ricercando soluzioni efficaci per una Educazione Linguistica inclusiva e attenta ai soggetti 'svantaggiati', che ponga attenzione all'intreccio di complesse dinamiche individuali, sociali, culturali ed economiche presenti sia nei percorsi formativi scolastici e universitari sia in quelli delle realtà di accoglienza, di reinsediamento e di mediazione.

Antonella Benucci è professoressa ordinaria di Didattica delle Lingue Moderne presso l'Università per Stranieri di Siena. È stata responsabile di progetti scientifici sull'intercomprensione e sulla linguistica educativa rivolta a contesti svantaggiati.

Giulia I. Grosso è ricercatrice in Didattica delle Lingue Moderne presso I'Università degli Studi di Cagliari. Si occupa da anni di progetti riguardanti la didattica in contesti migratori e ad apprendenti vulnerabili, in particolare nel contesto penitenziario.

Viola Monaci sta svolgendo il Dottorato di Ricerca presso l'Università per Stranieri di Siena sull'insegnamento/apprendimento nel contesto penitenziario in Italia e sul ruolo del contatto linguistico.

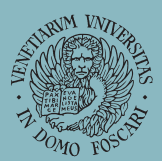

Università

Ca'Foscari Venezia

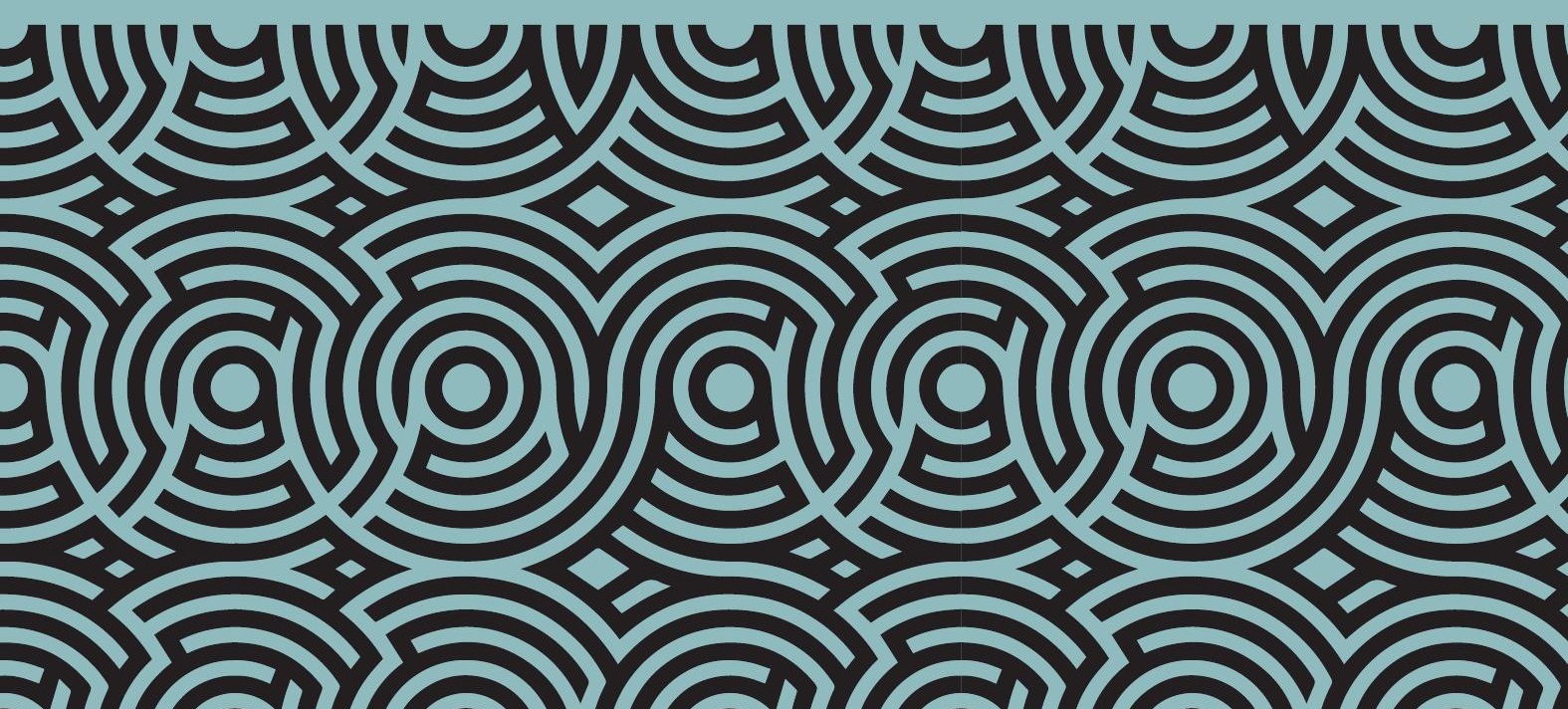

